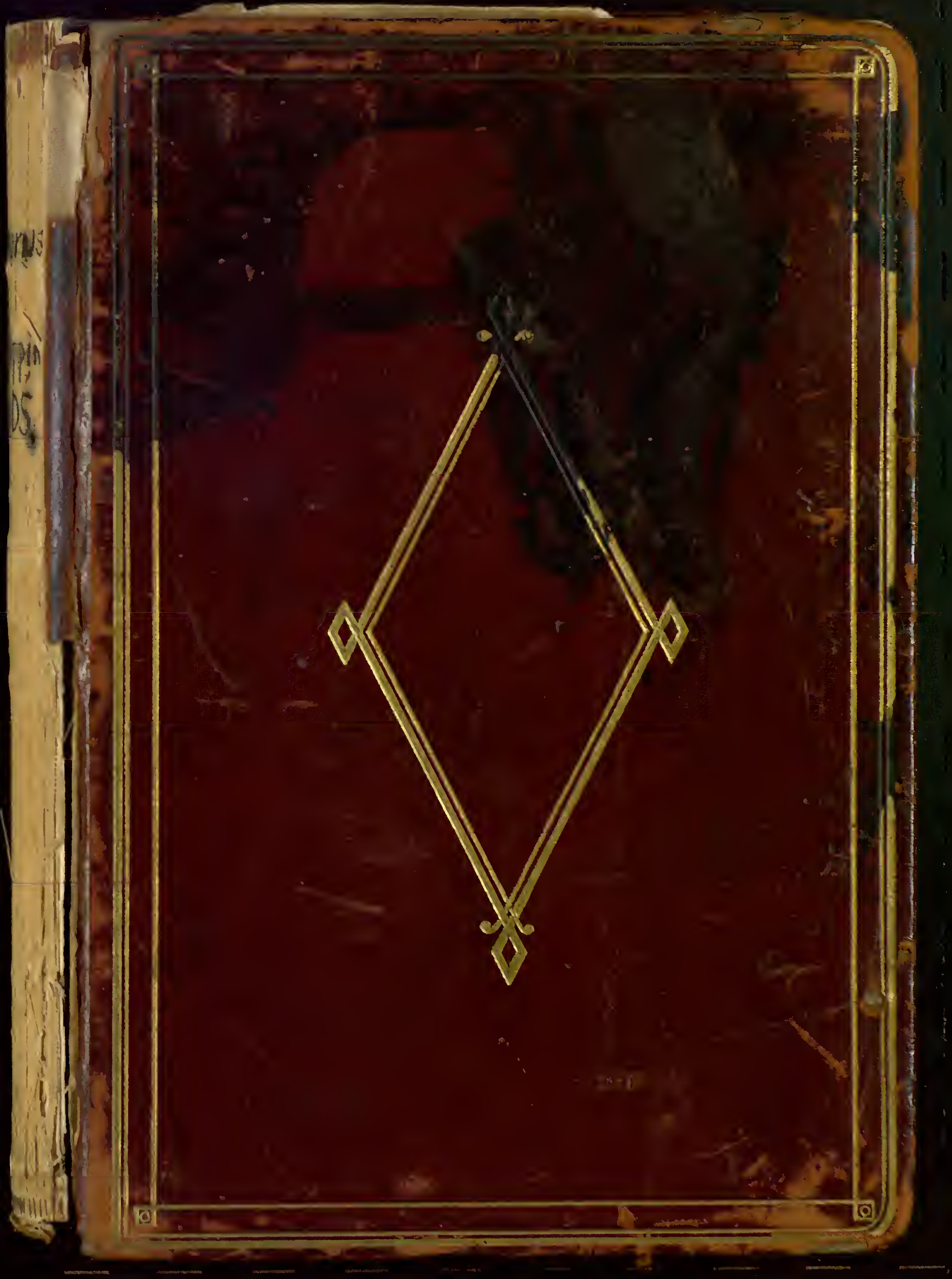




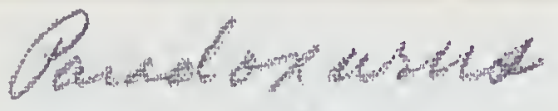

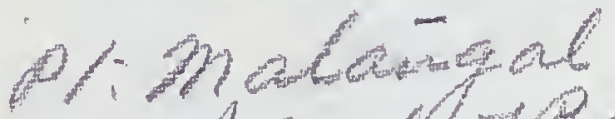

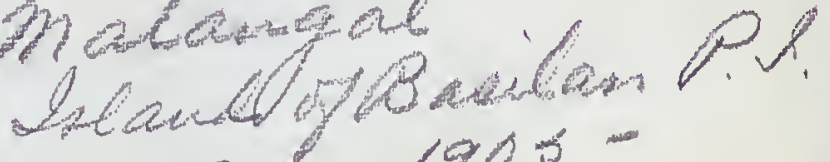

$$
\text { cumb , } 190 \text { क }
$$


WAR DEPARTMENT.

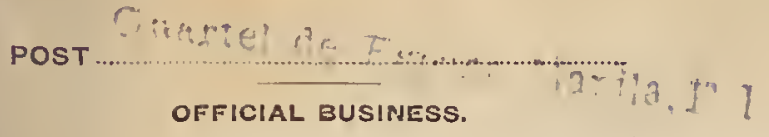

Penalty ior private use, $\$ 300$.

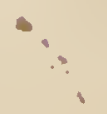

$\checkmark$ 


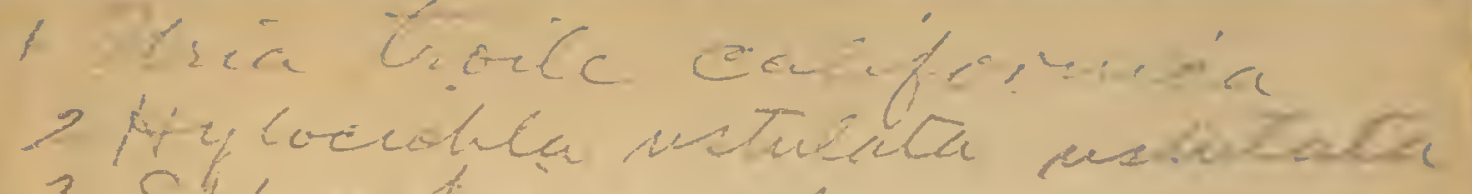

3 Sifipiaticion ruber

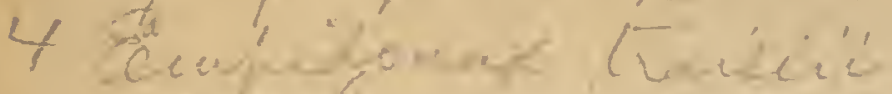

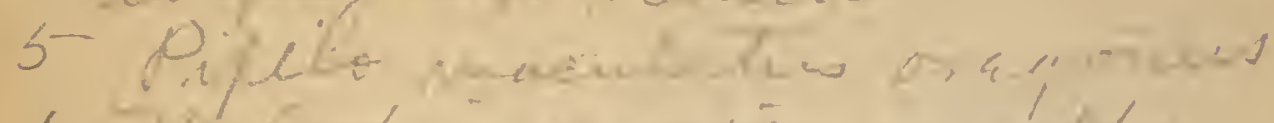

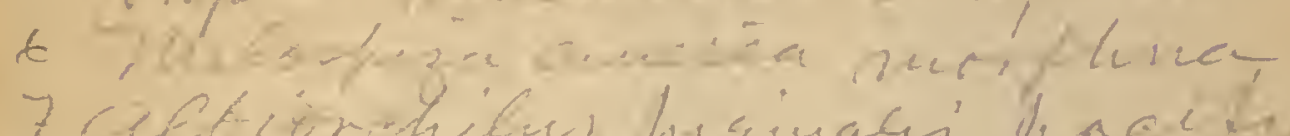

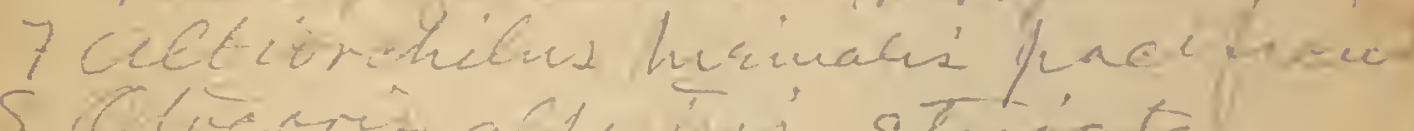

Sclicarit affenitur sticiata

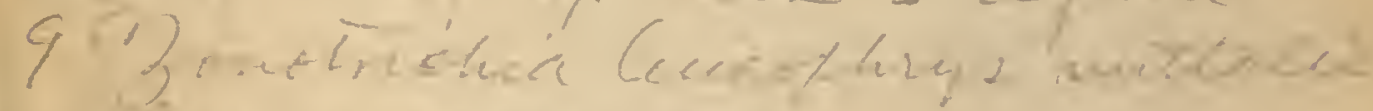

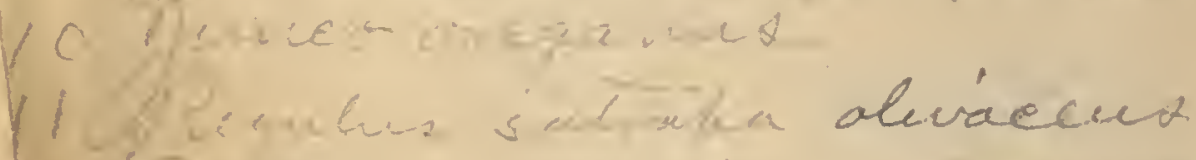

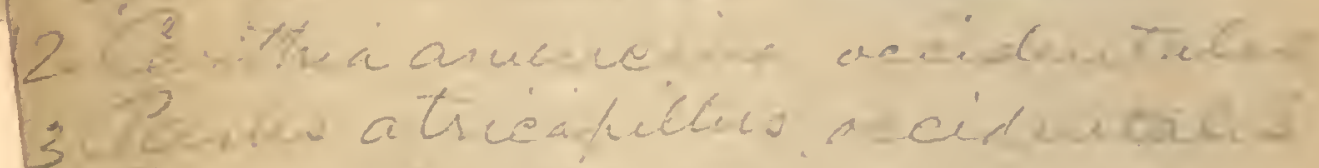

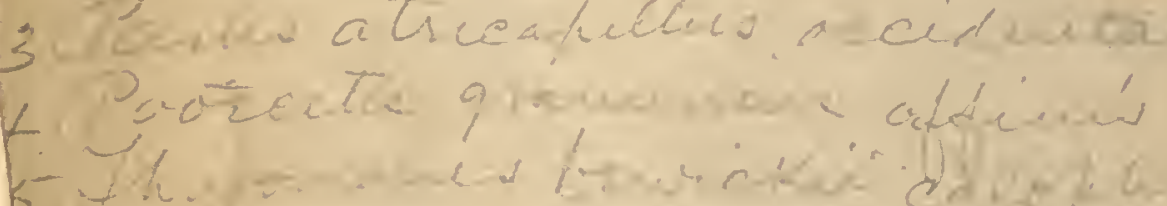

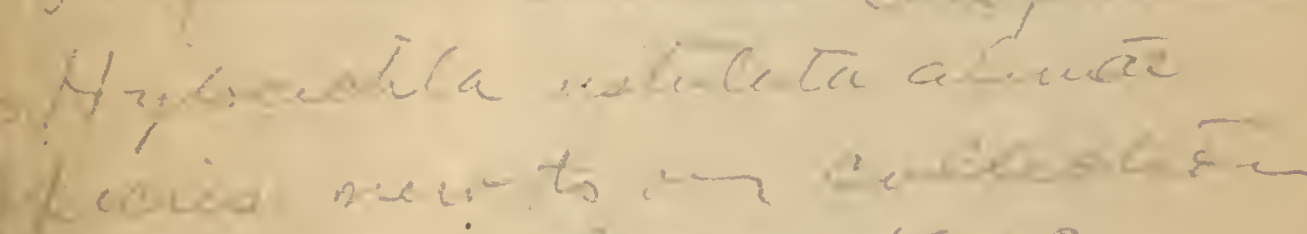

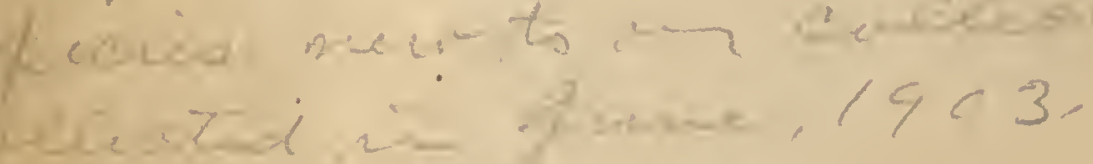


$\checkmark$

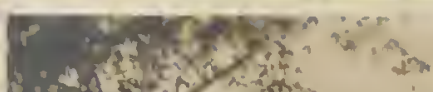

$P=x^{2} y^{2}+t^{2}+3$

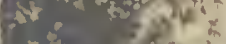

(2) -120040

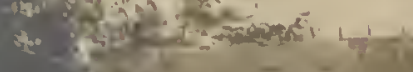

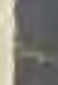

3

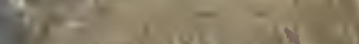

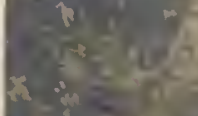

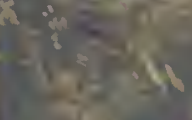

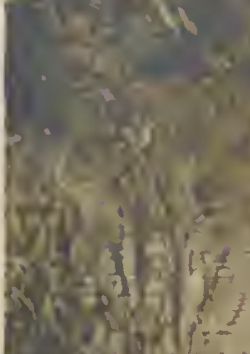

(1)

Fil

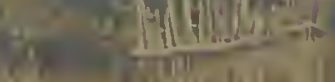

$X^{2}=$ a gong
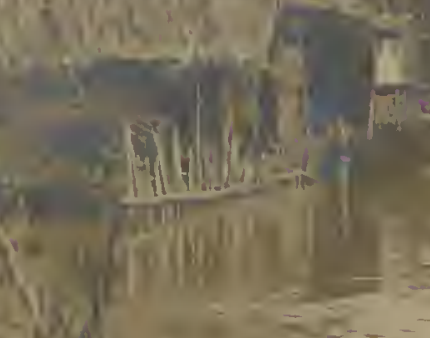

$\int_{i=2}^{\infty}$

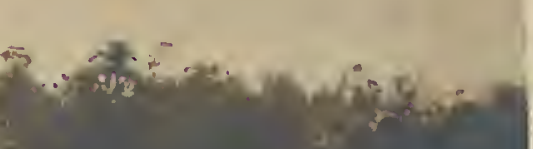

.

$\frac{3}{3}$

$\equiv+\frac{1}{2}=20$

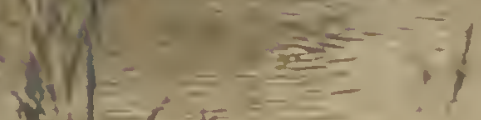

1.i $=1-5=$

(i) $: 14\}$

$=1$

$-1$

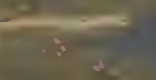

$+2$ 


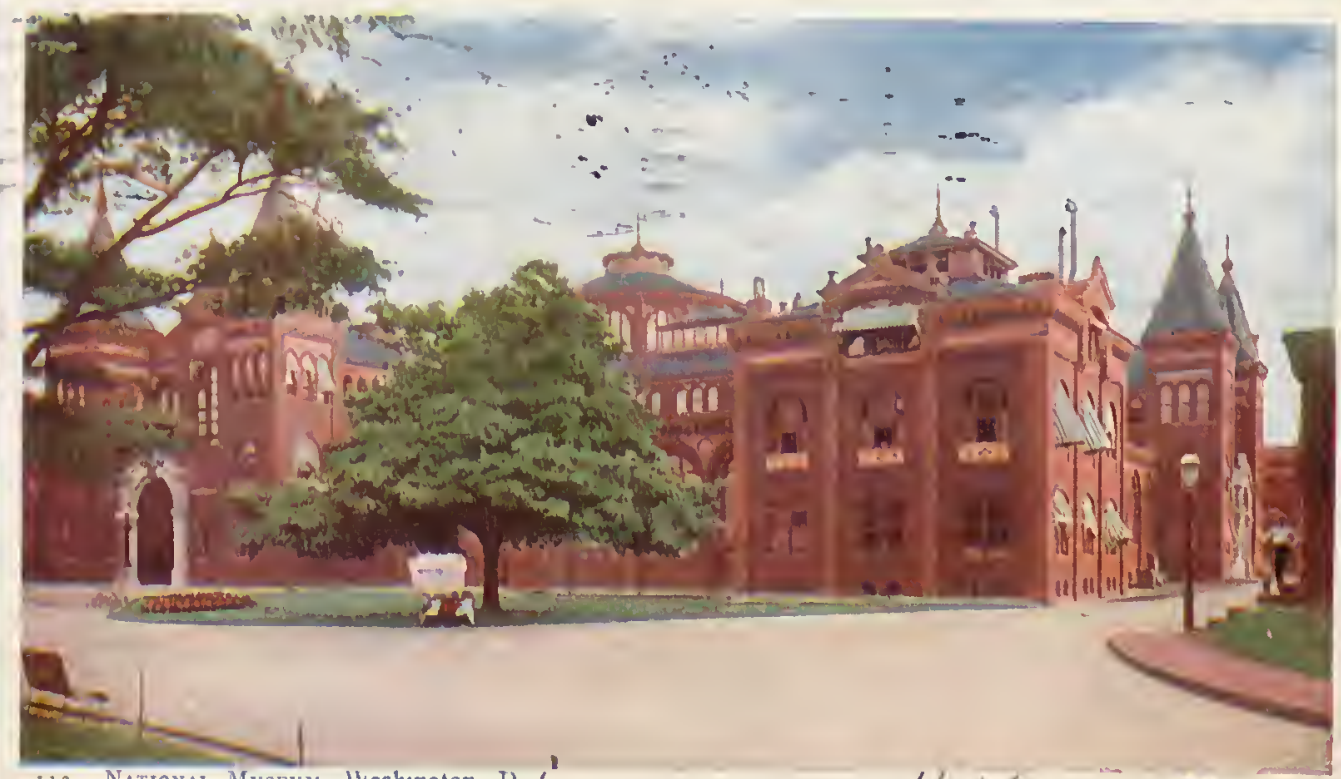

13. National Musfum, Washangton, i. 


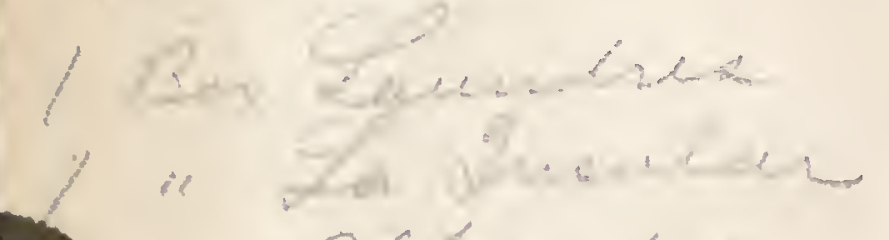

D. Alkowota

sec clarox EQ M.Marus

4 
Jan 27 2sabellu

" 28 Fich cuert

" 29 "2bid" creek. Hydracerrax speemin

"30 "Old" Kkrlef near vecare.

31 Old Camp,

fiebr. 1. Sheam / daip mench ap. cutors.

" 2 " 2 .

"3 ced camp off hutr. of

Dier.

"4 Left Basilan. 
CARTE POSTALE

Postkarte_-Post Card CARTOLINA POSTALE-LEVELEZ6-T AP l'ocztówka-Bricfliaart-Dopisnice Karta korespondencyjna - Tarjeta postal Открытое Письмо

Union postale universelo Brefkort.

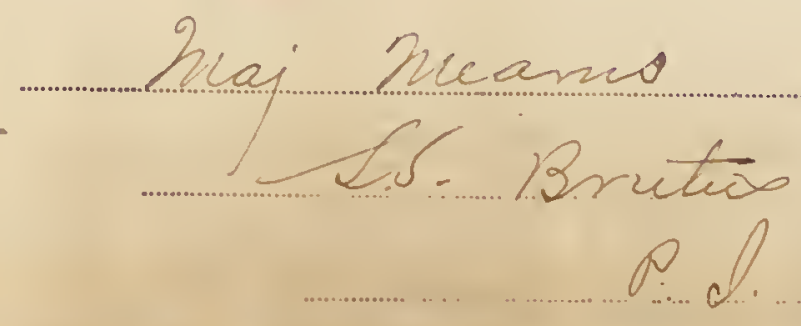




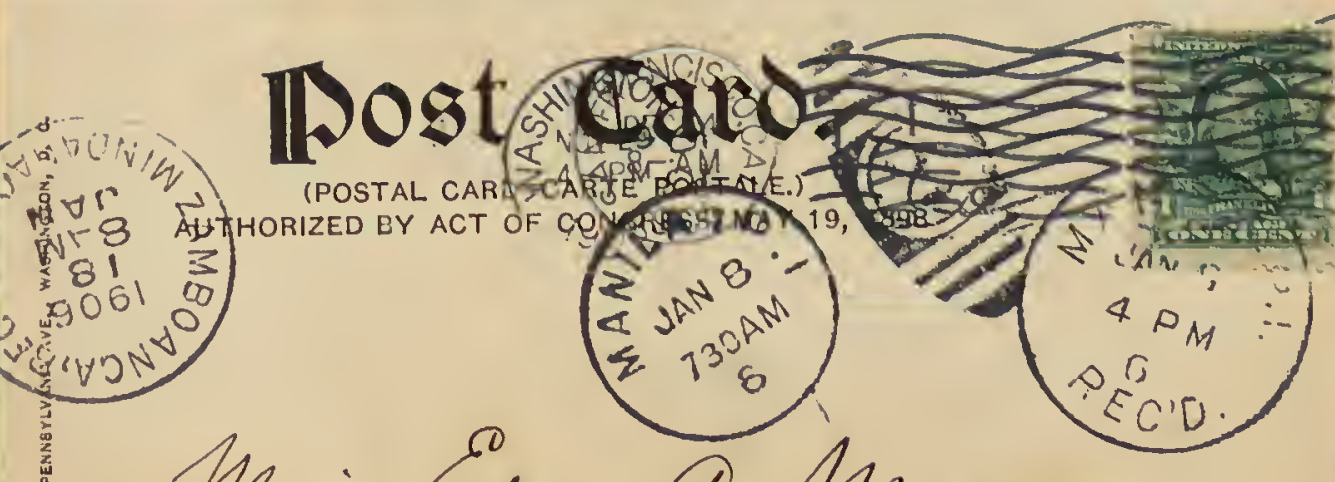

Major Gdejar A Mearns Surgum a 8 Amm Sambeangm Atarila 


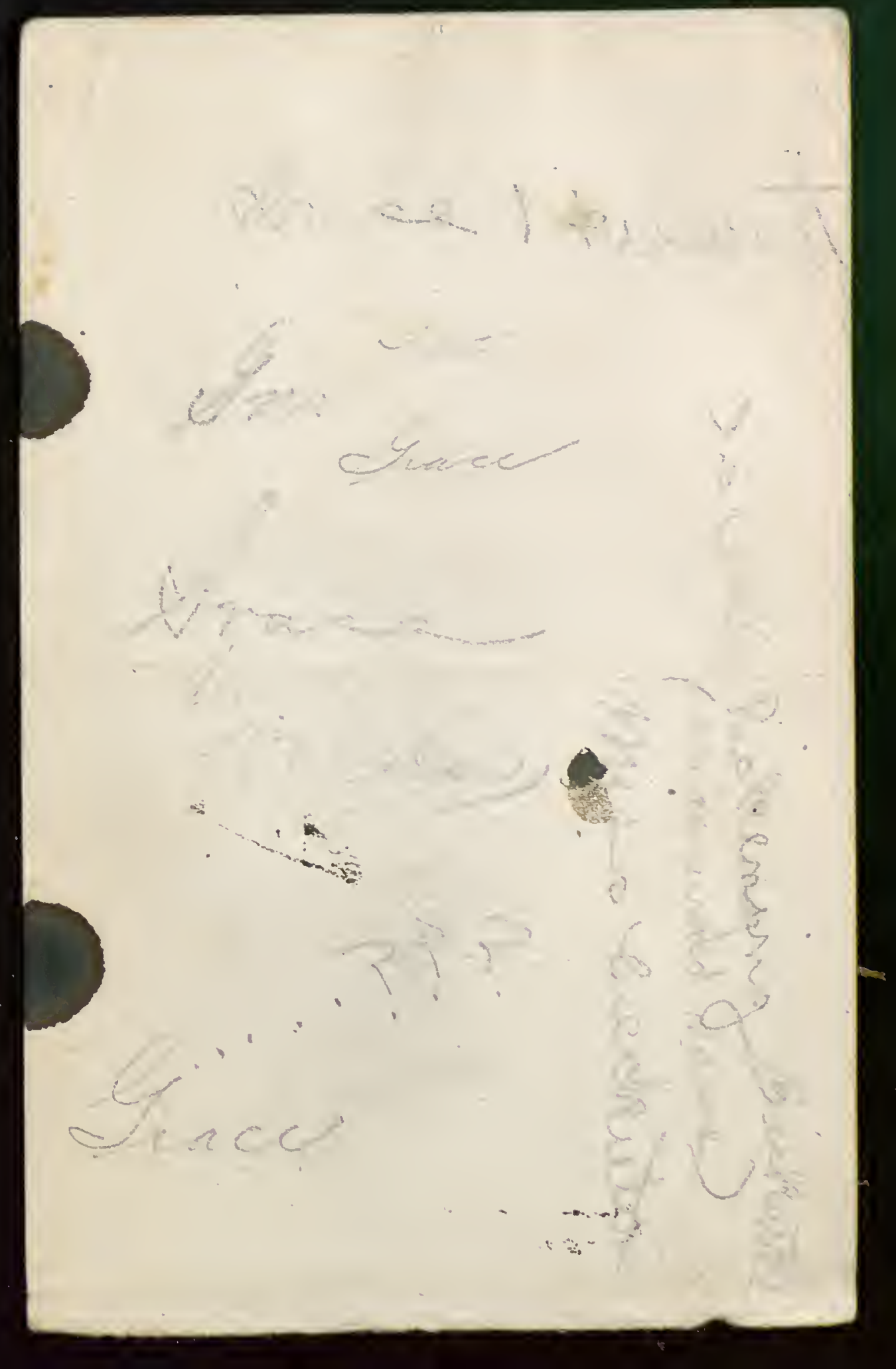




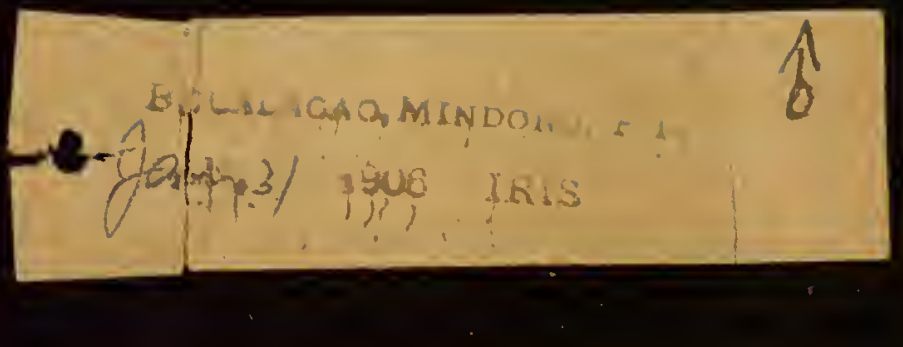




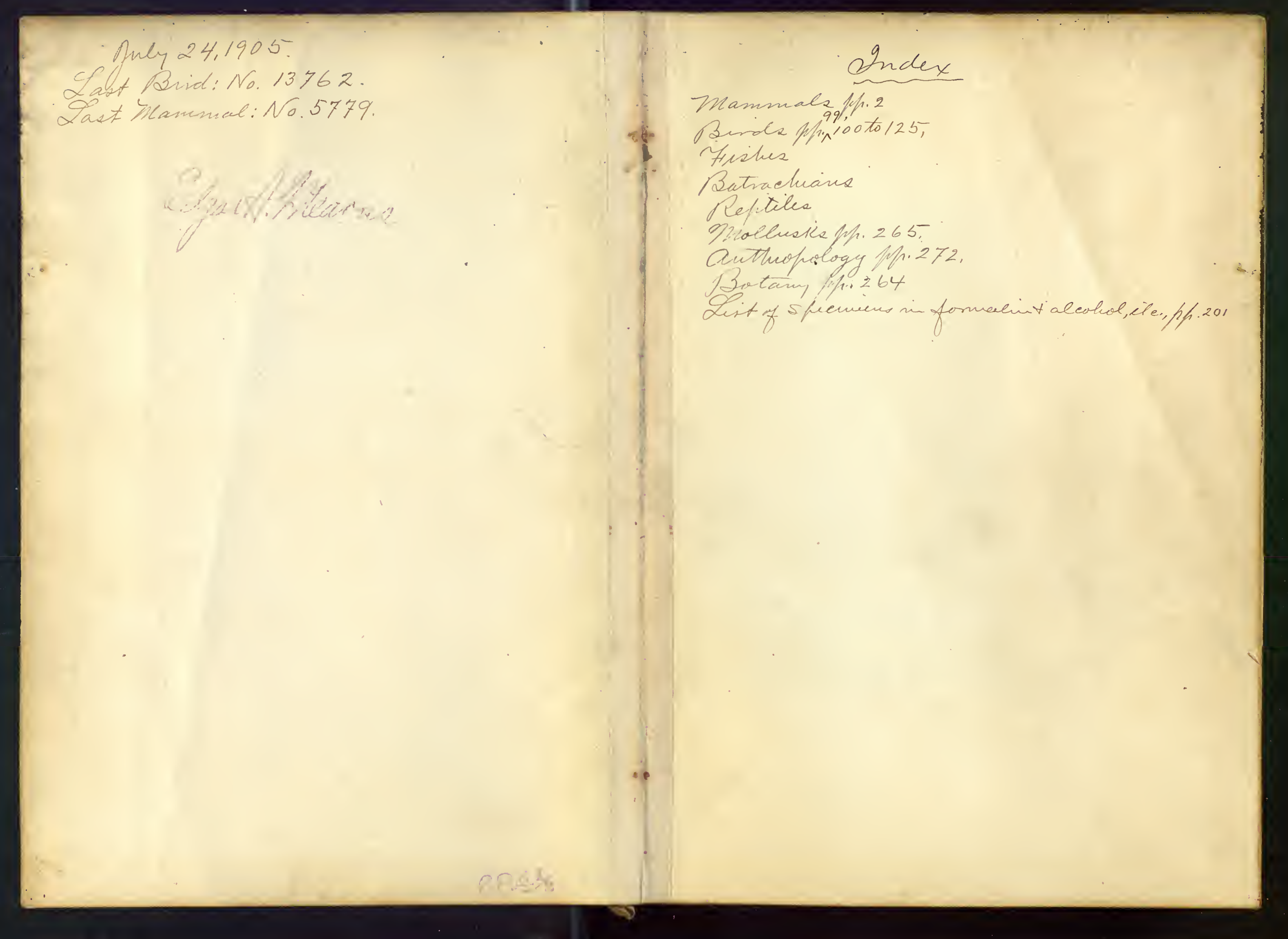


Cecranut Bucth = Batgahngan (Estritlus) lim-pe'-a = Eentipede. 


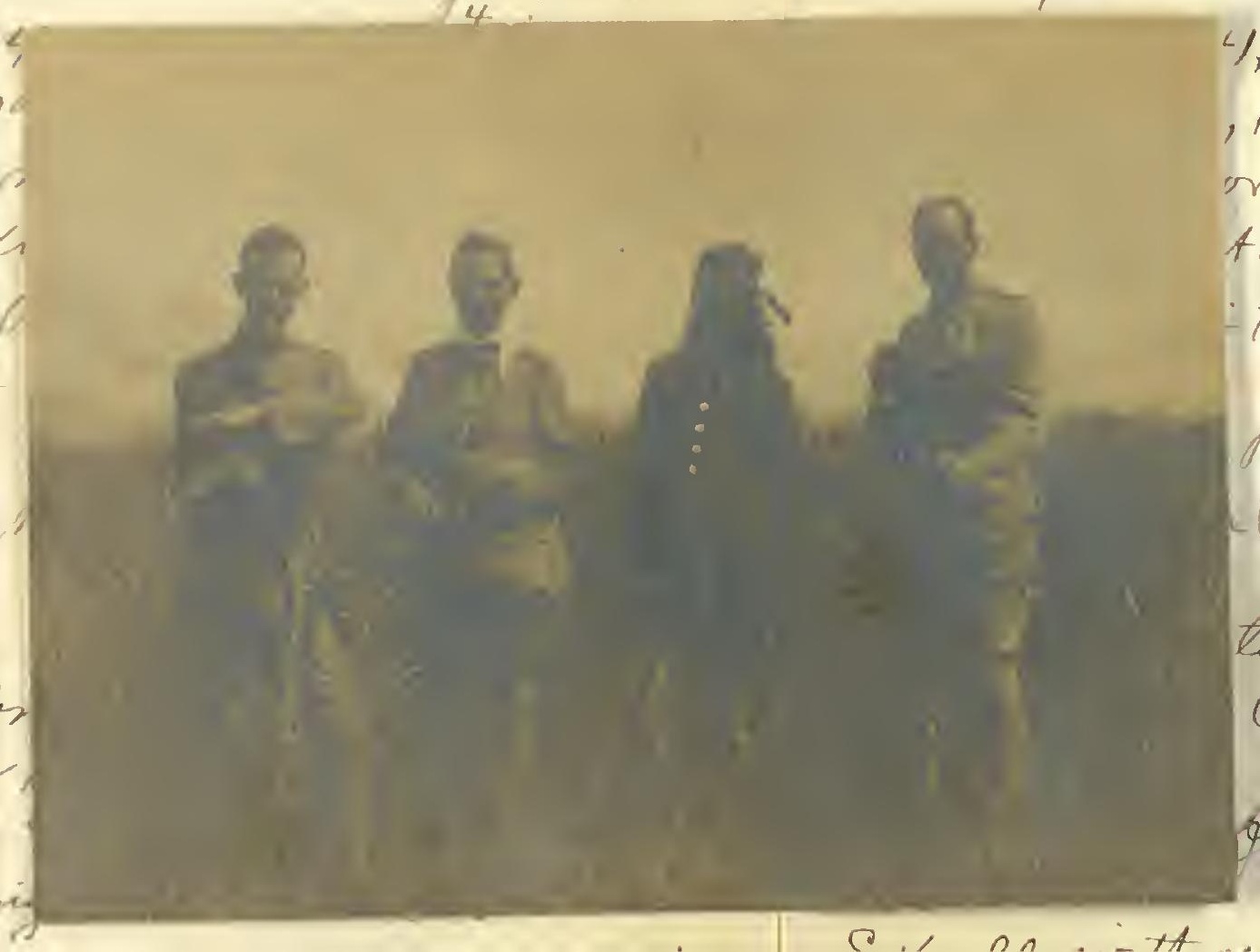


2 Seland of L 1 man, Paifie Ocean.

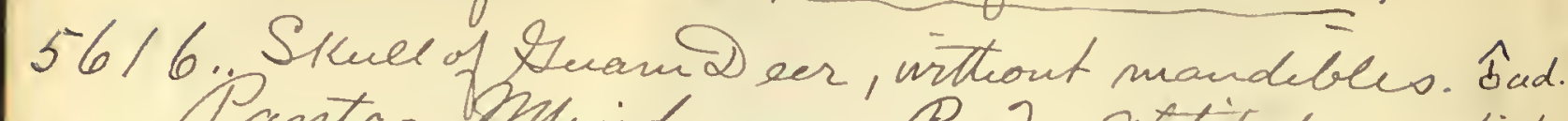
Santar, Mplenidanao, P. D. Attilude 1907 fict. 5617 . Mus Pantar, Mindanao \&ad 410;

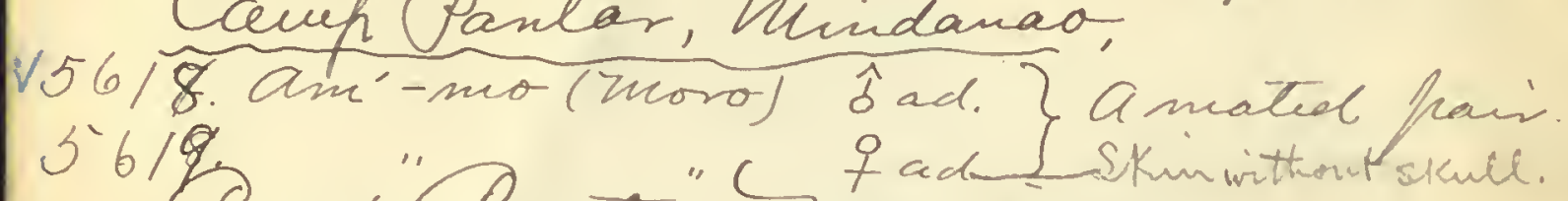
5"619 Canf "Pantai," Huidanas.

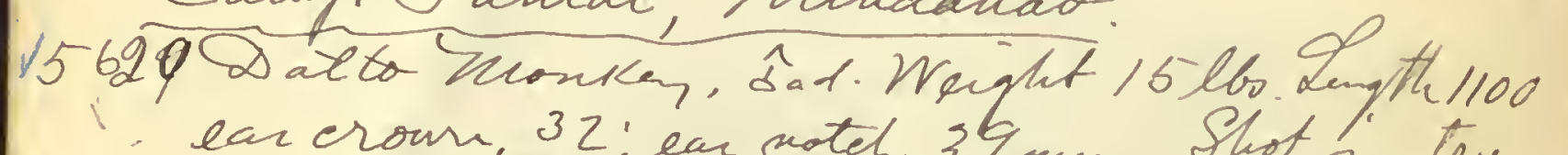

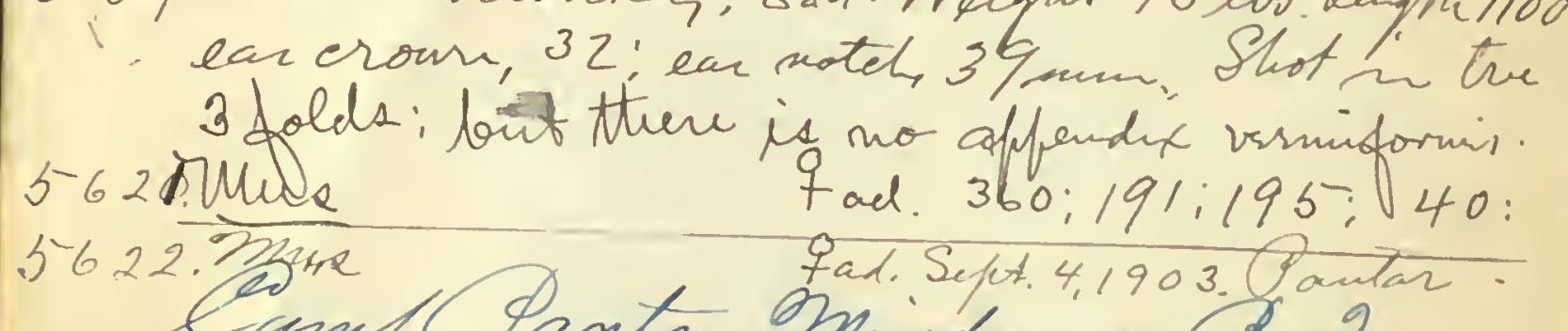

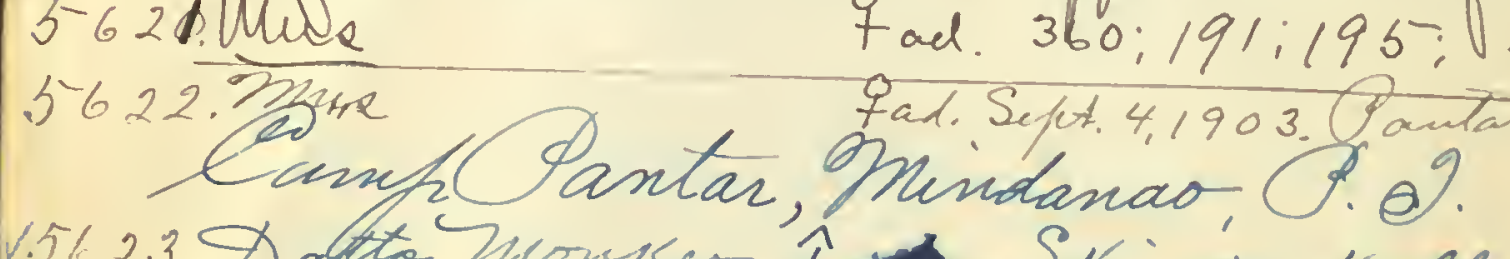

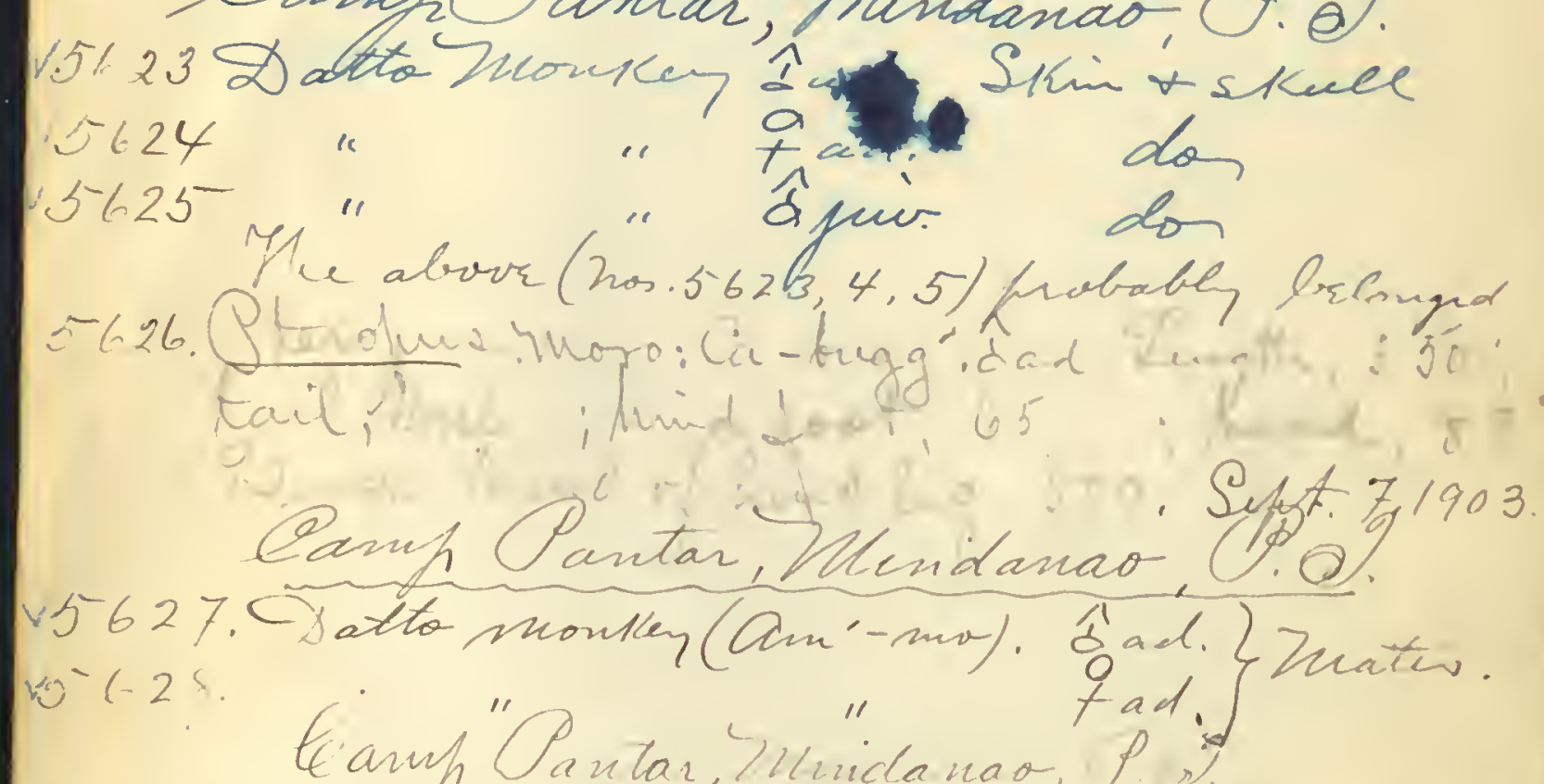

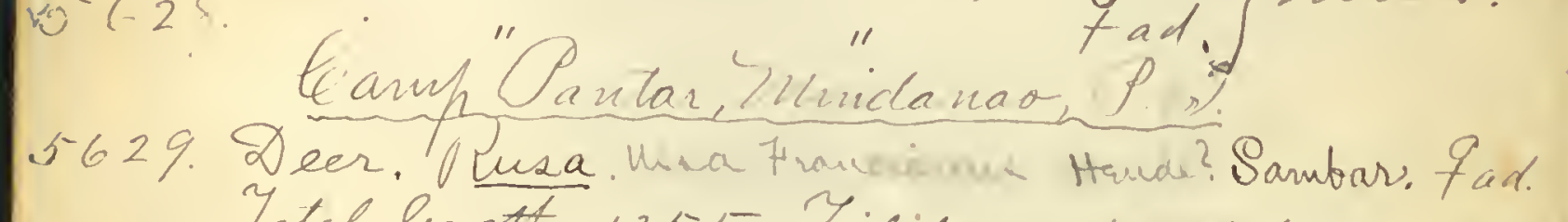

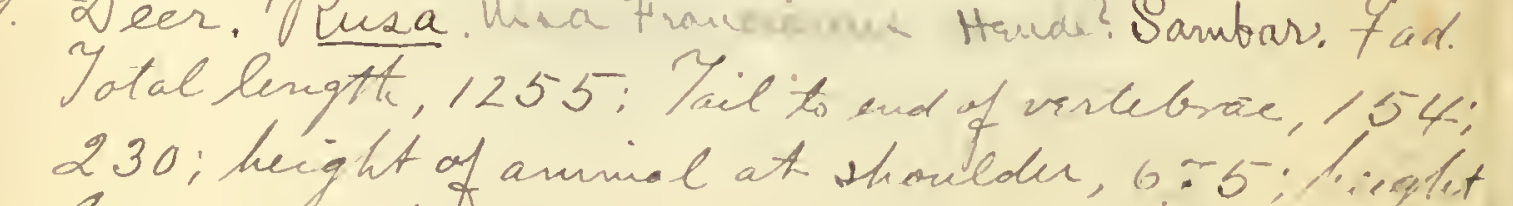

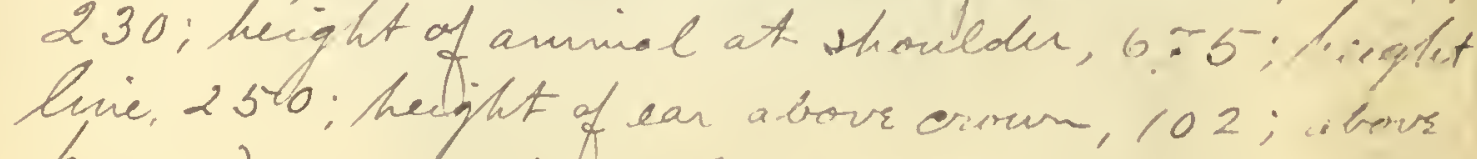

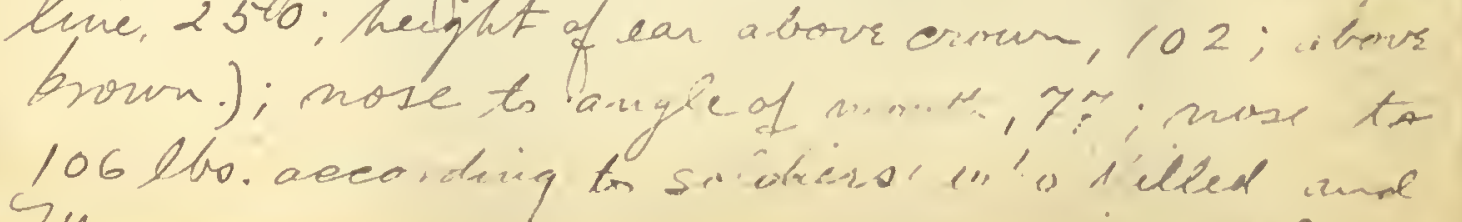

207:211:40:53: Onquat 18,1903.

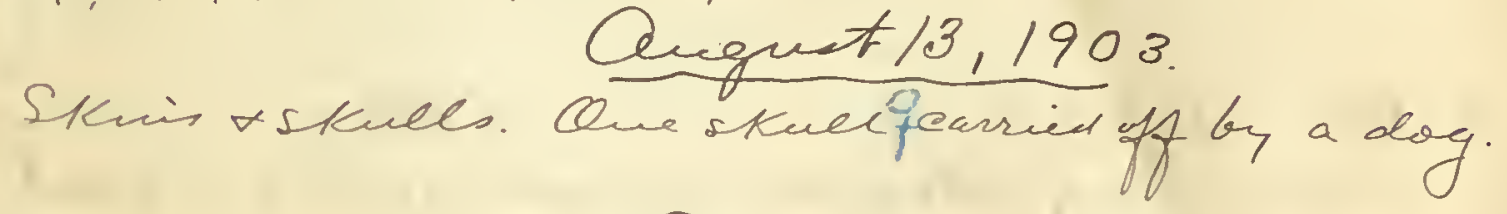
Quguat.26,1903.

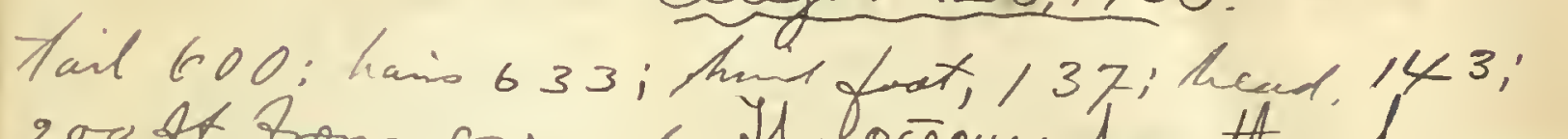
200 ft. From grond. The eâecum then th human 38;16; 21. Woro mane Re'-ah.

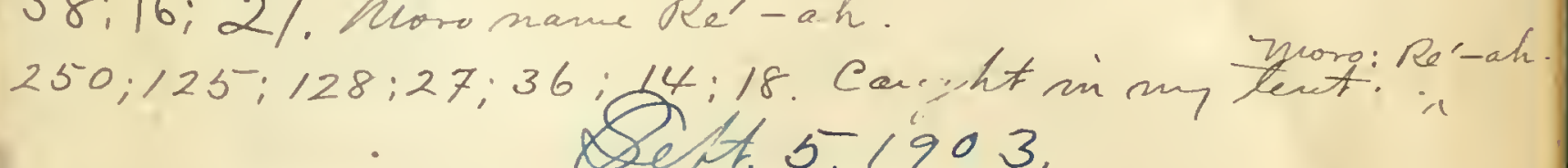

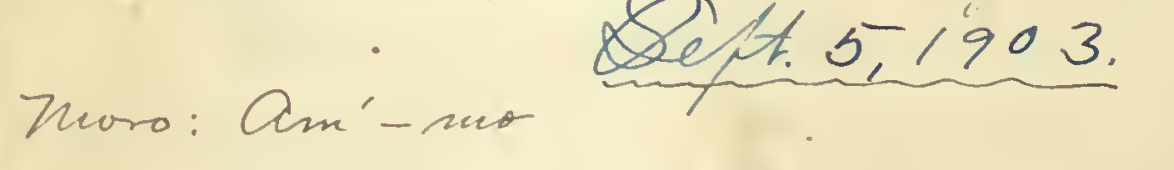

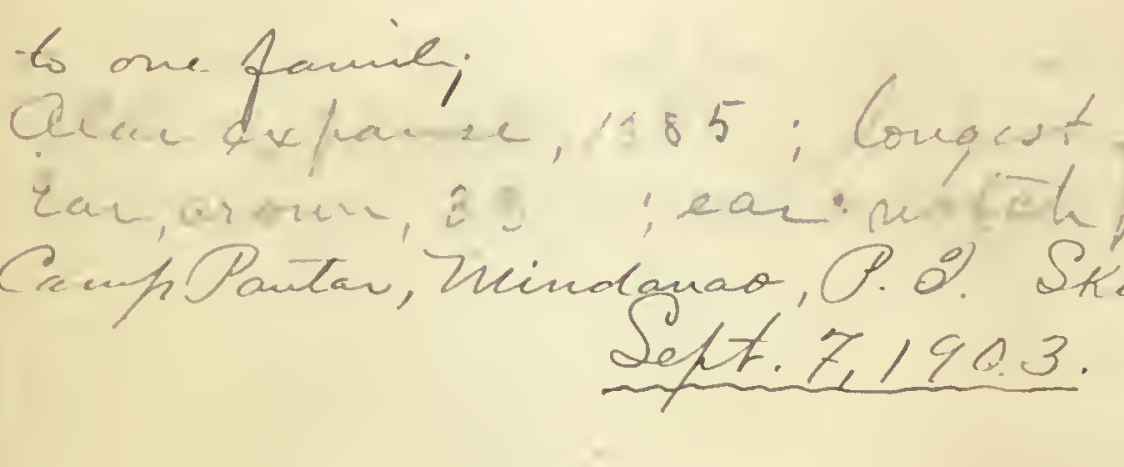

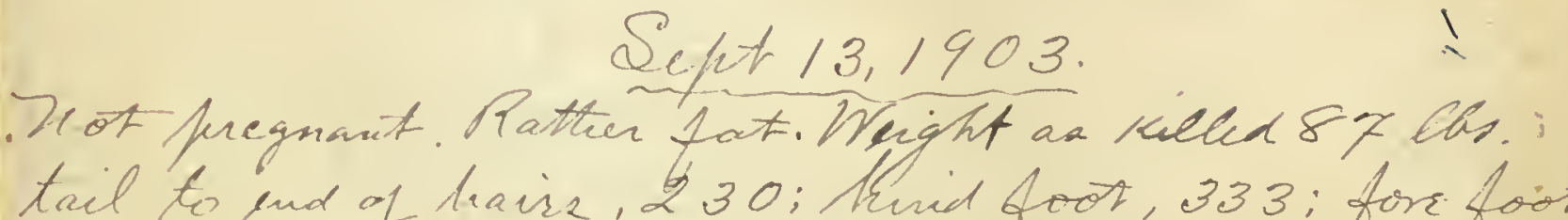
tail to und of hairs, 230: third foot, 333; for foot. moteh, 96: deameter of eje, 20, (leolor of eje, dane

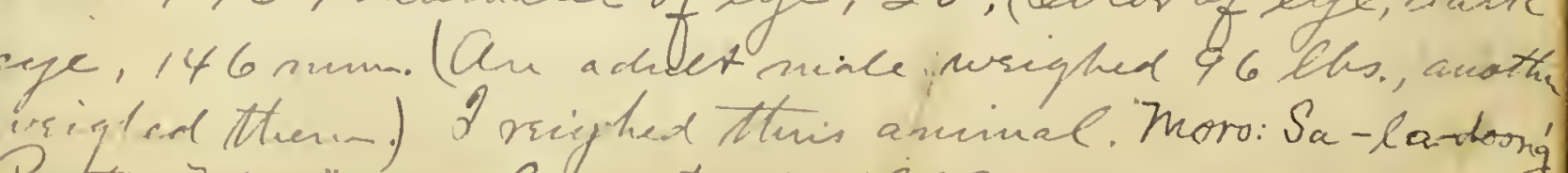


4 Camp Pantar Onindanao.

5630 . a. Thu 5631 .

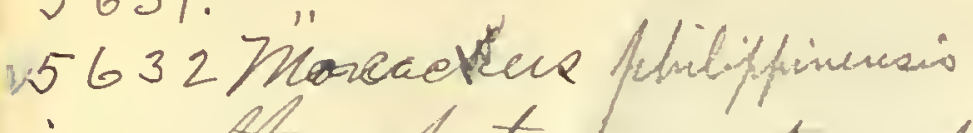

Ah 1 to g jur.

5633 . Homo safiens (Vhor). dad. whom then altoeked ncar carrp. The Sergeant but with gw. Th, sears. Yhe other Bergent klilid

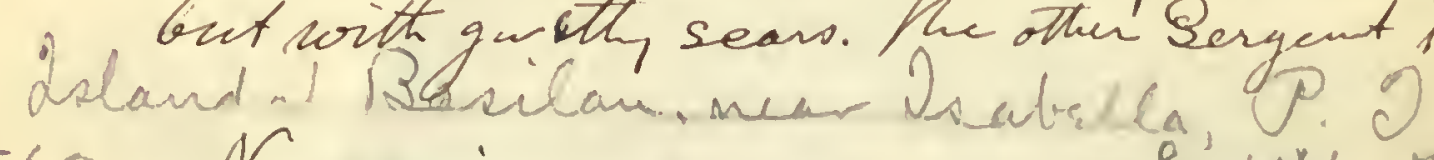
5634 . Nannosciurus $\quad+9,166 ; 7 \vec{i}$

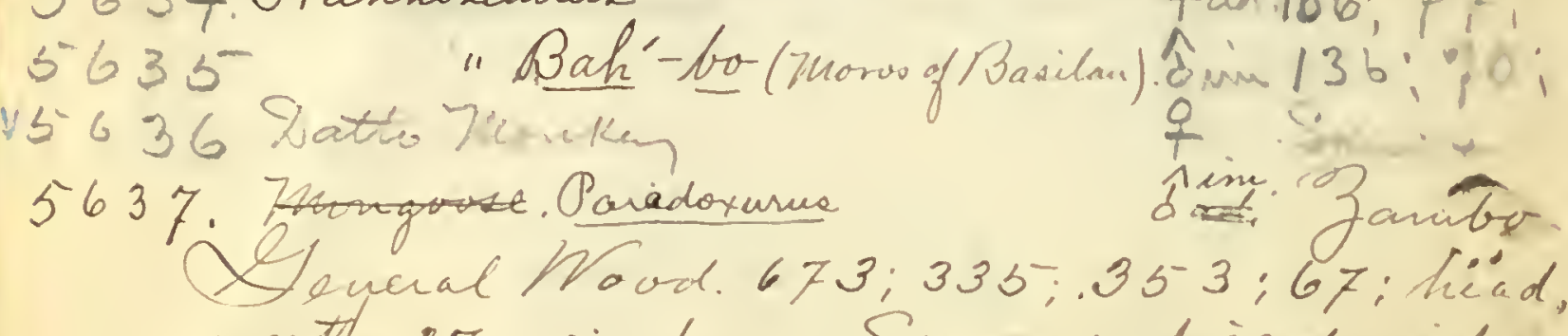

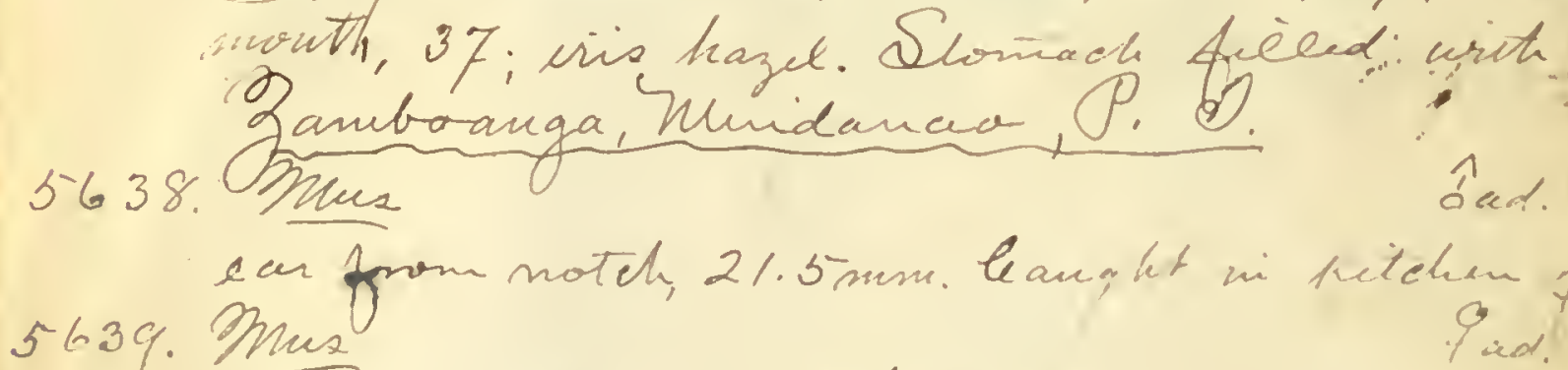

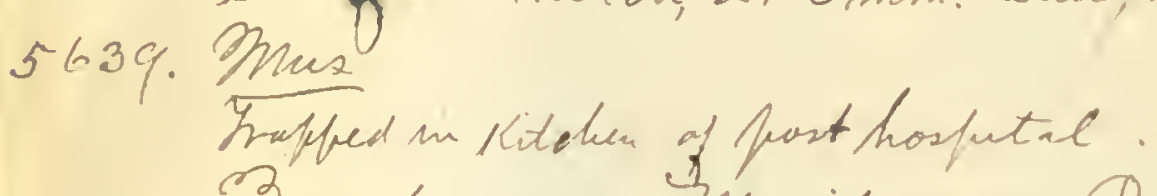

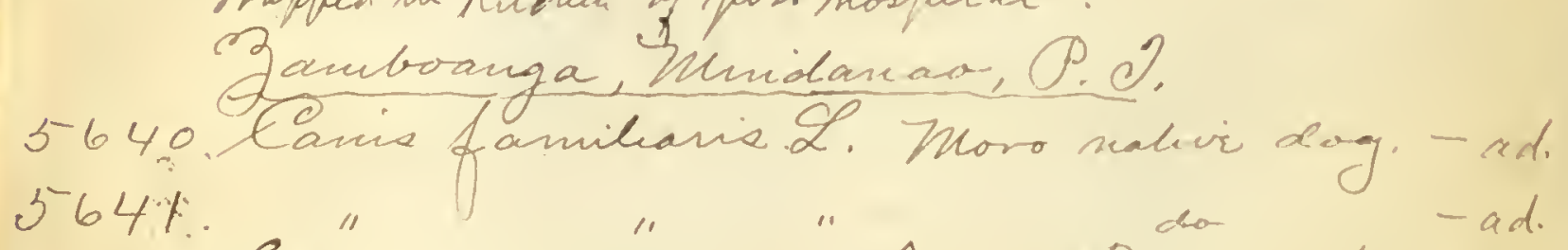
$\$ 642$. Teerver fhiliffinus. $\hat{b}$ ad. Pari of horne, 5643 .

5644. 5645 . 5646 $5-647$ 5648 $564 \%$

ốad. One "horn.

$\hat{a} a d$.

$\hat{d}$ ad.

$\hat{a} a d$.

âad.
Sefet. $20,1903$.

5

390:196:200:40:52:14:21; deametio of eye, 6.5. $377: 185: 189: 41 ; 54: 17: 23$; Majoir Aale and 2 ate some fit and found it edible,
Srim salted. Scull cleaved.

Que of three hioros who cut nif ane of two Sergeants who pros choffed with a canifelon everitualh recovered th Dalto and two of his slaves.

Jan.26,1904

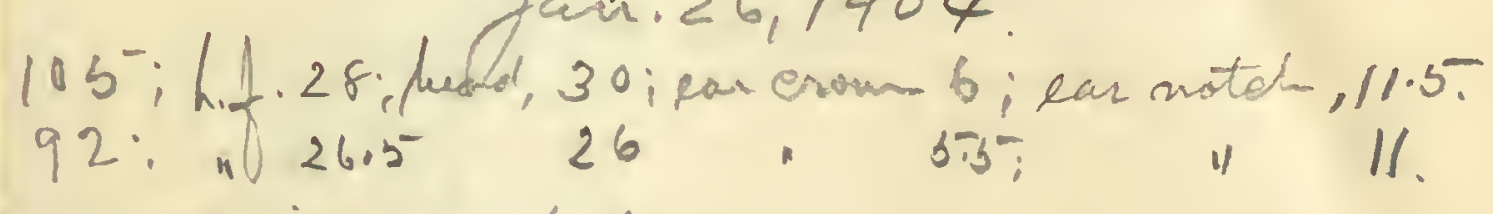

stude in acarbol.

mag, Windanas, P.d. Febr. 29,1904. Sent me by on: car erours, 36: ear noteh, 40 ; nose to angle of clicken meat.

Manuary 15, 1904.

420; 201: hind folot, 43; head, 56.5; ear from croum, 18; post hosfital. Osy stuel altached to skini? 405; 195; 199:42;53:18; 22. Mono: Ahm-bough'. Domstull all ited to sikin.

Skuce. Hetoncen 29:1904.

Skull withont mandibees.

Year 1903 with. part of sicuel.

Year 1903 
6 Dato Olin fort at Serenaya marsh,

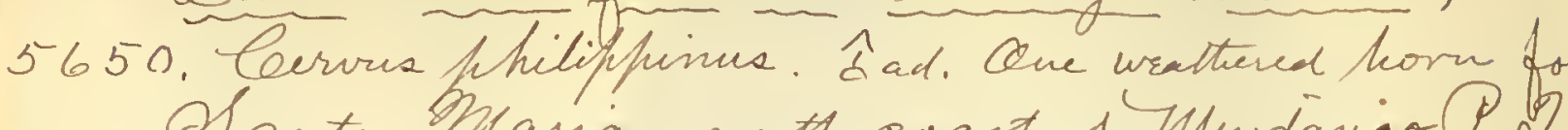

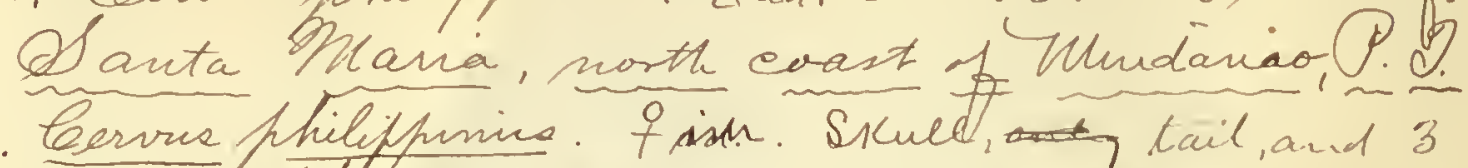

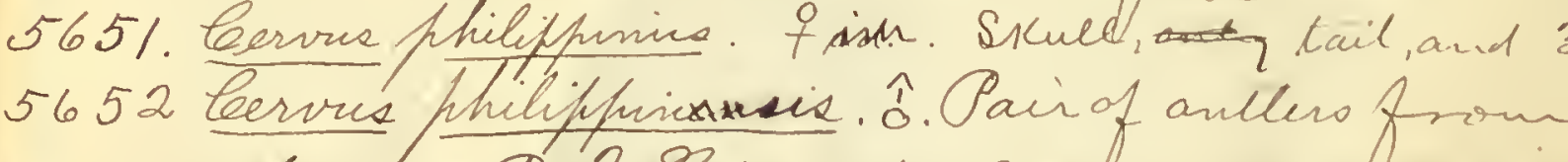
- danaw, D. e. Pray 25, 1904 .

5653. Snacl dact-colored But. ô ad. leaught by a eat 5654. Znux kantarensis? Inder a small tree,

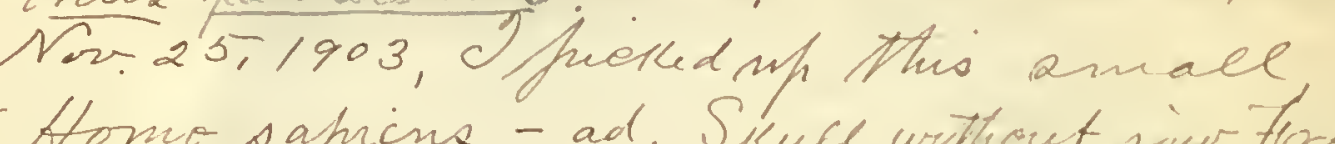
5655. Homo saprine, - ad. Skull wothout jaew, firem

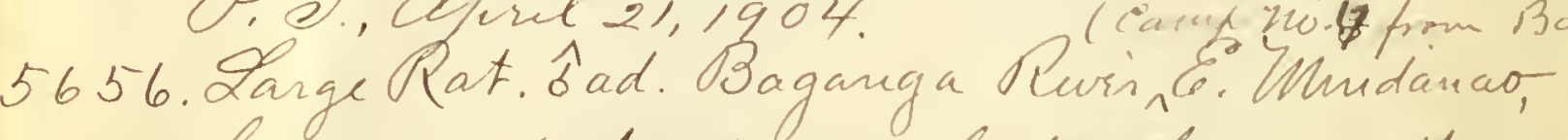
large roek, broich smy bed and nem the Ond poisoned the steni, which was dreed in $565 \%$. Peirt. Uiverra Salauan, ncar the foothills, on lift Nole. The mannmale of the Mut. Apo tory 5741. Amall Bat. Aad. Leollabato, Rio Lvande, the Oundanas, lynig at the doek. In aten= 5743 . - ad. Sua 5744 . Ceervire fhiliffumis. Ead. Pair of horns. 5746 . 5747 5748

sad.

sad. Onad. One horn/shed).
Rio Asrande Valley, Sundanad, P. 9. March 11, 1907. Afobruary 17.1904.

Simpitan, Ali's bätleground nean Lake Bulvan, Min= at Gamboranga, Mindanav, P. Y., May 31,1904. Frormalin.

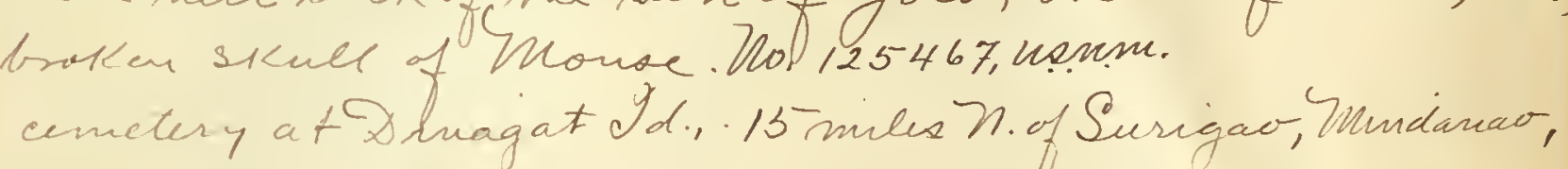
ganfa on the cranth, baited with frie foriod nuat suvi. The tip or aprial of tail was whiti. Istmicest. plonst paper, but altareced by anted Sreir and. Srucl Q.R. very destruchere to sureat fotators (comoti). The Mandayans (Skule lift at Bazayan and loot.) Sicin oney. fork of Soraca Riven, Lake Lavar Basin, Murdanas, P. . Nos. 5658 to 5739 will br entered subsequentty, The. Celeristeani Ode, from his hemp sanch s miles

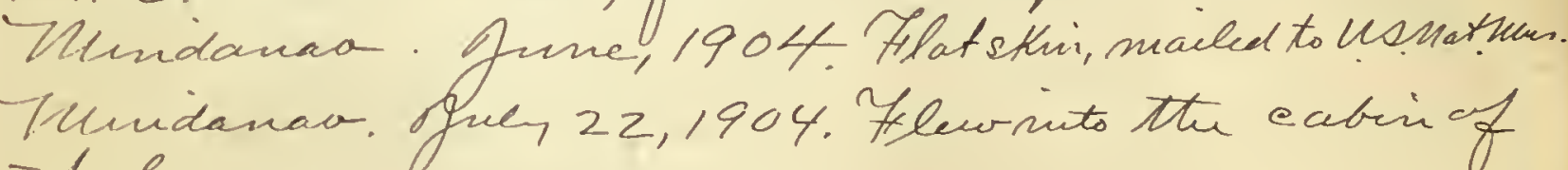
SHolle without jaw. Jamboanga, Inind, P. 2. 1903. Stinel.

Zamboriga, Mnidanao, P. P.

do

do

do 
3

9

5749. Bat

Fad. Zamboanga, Mnidanao, P. 2. Aug. 11904. Aleoholic.

25750. Mnowkey sad Laland of Sulu, P. ?

$575 \%$ Mouse

- ad.

Novanber 27, 1903. From stomach of Hawk.

5752. Mhe

Hind foot, 37. (Numbur 5732 dufleieated).

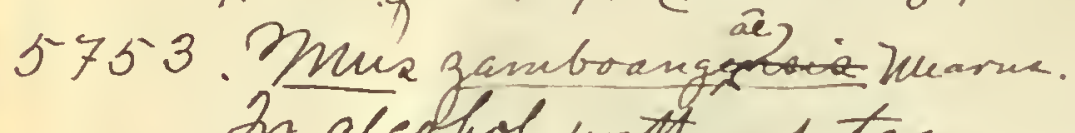

In alcolool without tag.

5754 . Surall Bat.

In aleohal. Hetal tag no. 166 .

$\hat{b}$ ad

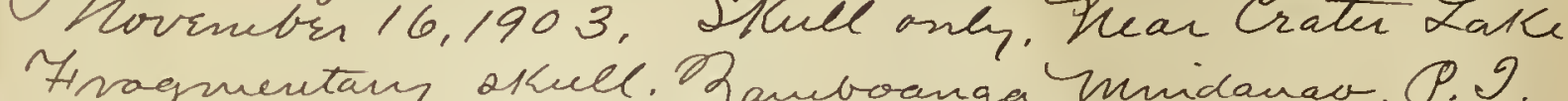

Cat. Ho. 125466 , usns.

Stin and skull. Davao, Mindanao, P. . . Fuly 19, 1904.

bad. Gamboanga, Mindanav, P. O. Panuary 20,1904.

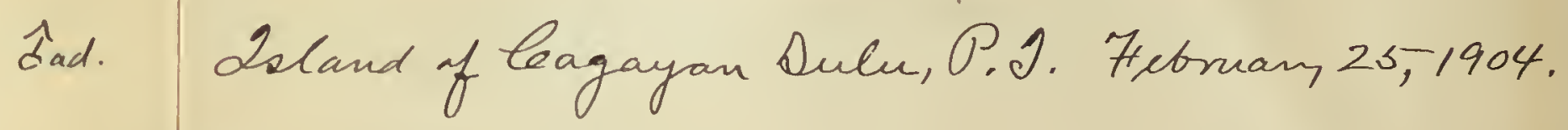

5755. Smaller Frritt Bat. Pteropua eagayanencis.

5756 .

ho. 125289 uenum. $\hat{b} a d$.

do

5757

- 125290 . $\hat{\delta} a d$.

dom

5758 . Since if evingete

. 125291 "quad. do

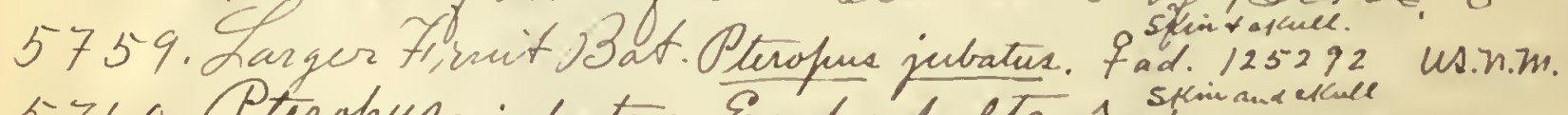

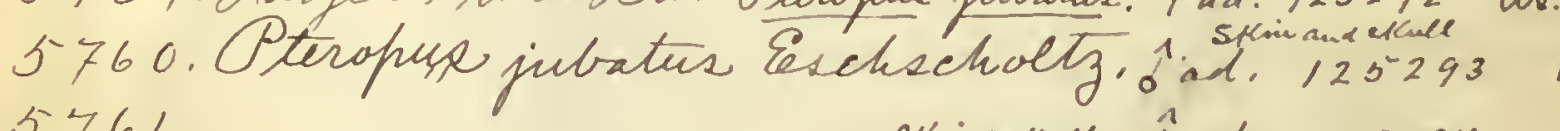

5761.

5762 .

5763

5764 .

5.765 .

5766.

5767.

5768 .

5769

5770.

5771. Macacus "stinitatuel. ôad. 125294

"Sthin (stuclesinde). fad.125295

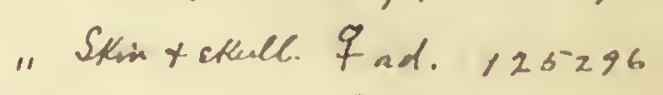

tad. 125297

diad. 125298

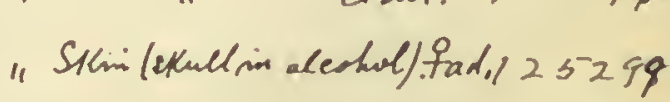

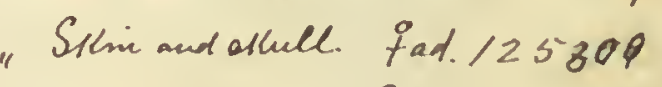

tad. 125306

íad.125302"

$\hat{o}$ ad. $12530 \mathbb{k}^{3} "$

$\left(\begin{array}{ll}25325) \\ \text { and }\end{array}\right.$

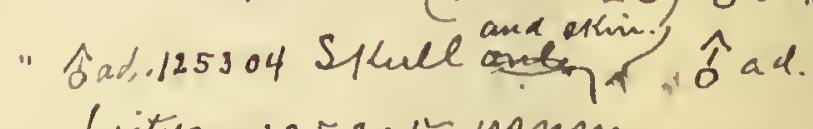

"foitur. 125305', nomm. 5773

5774

"

". 125306
Cagayan Sulu 2aland, Sulu Sea, P. 2., 7. eb. 25,1904. Banga 2sland, if \& sice of Mindanao, 7fer.1, 1904. do Hortue of one of abover fernake. 


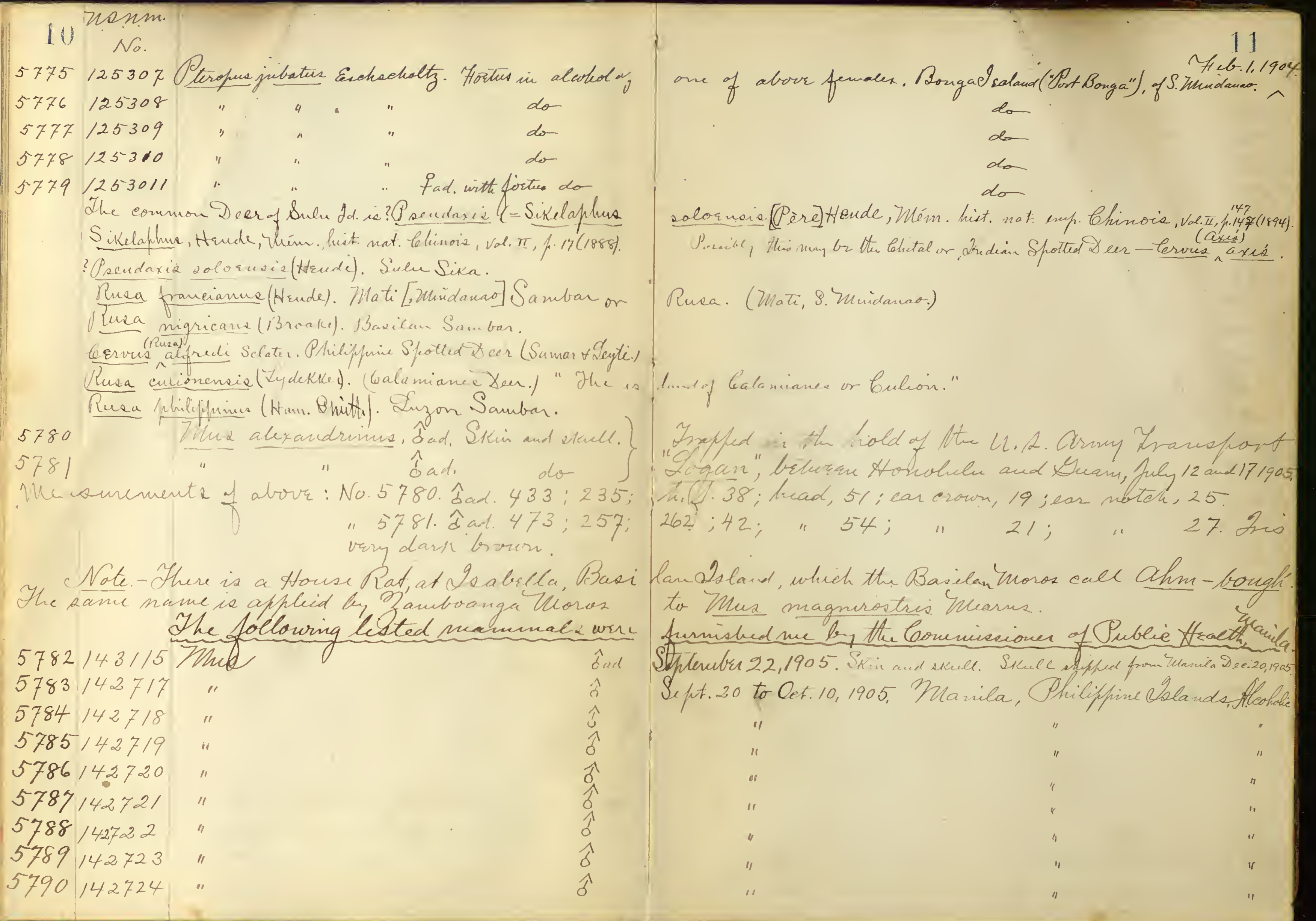



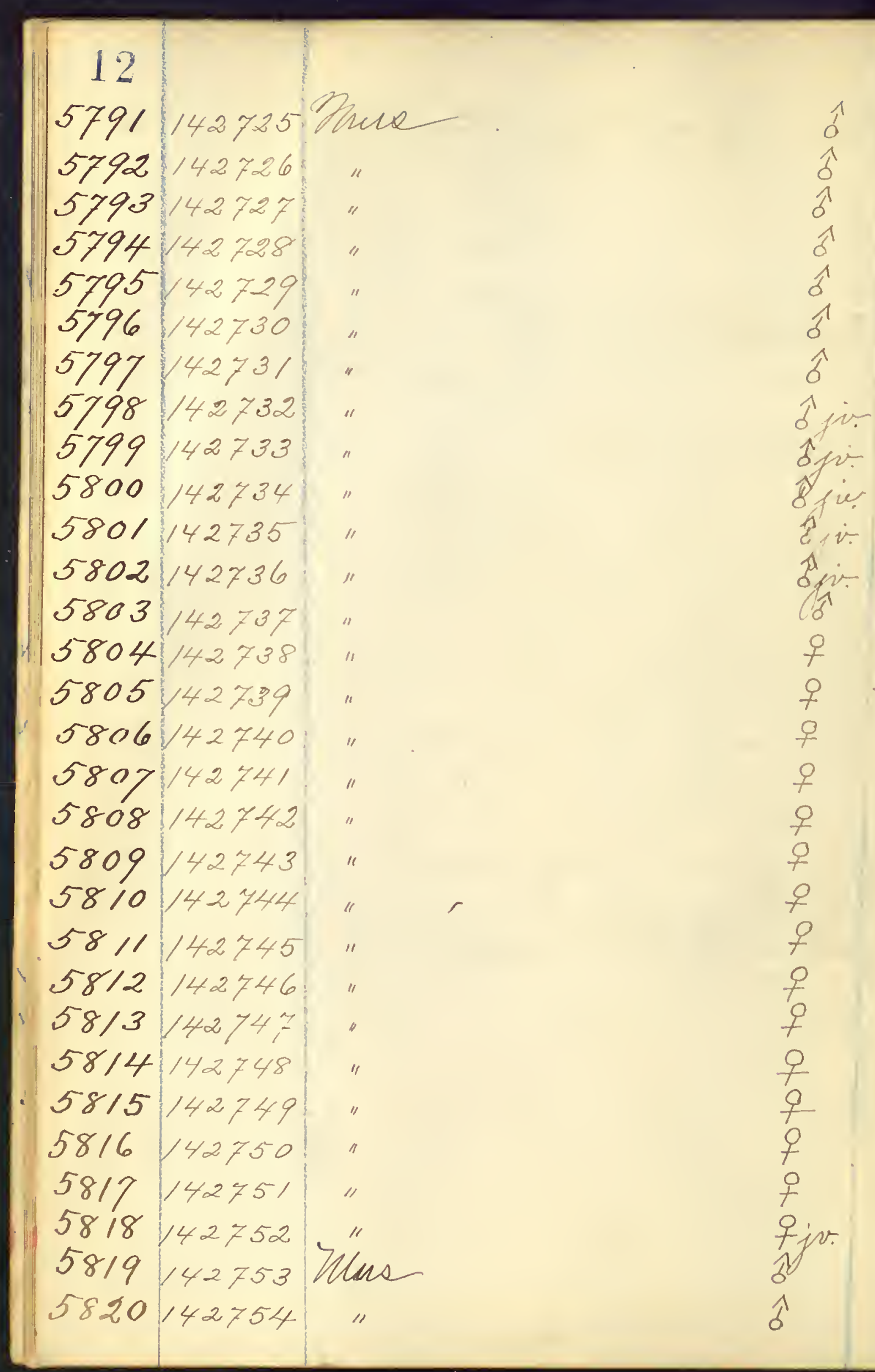







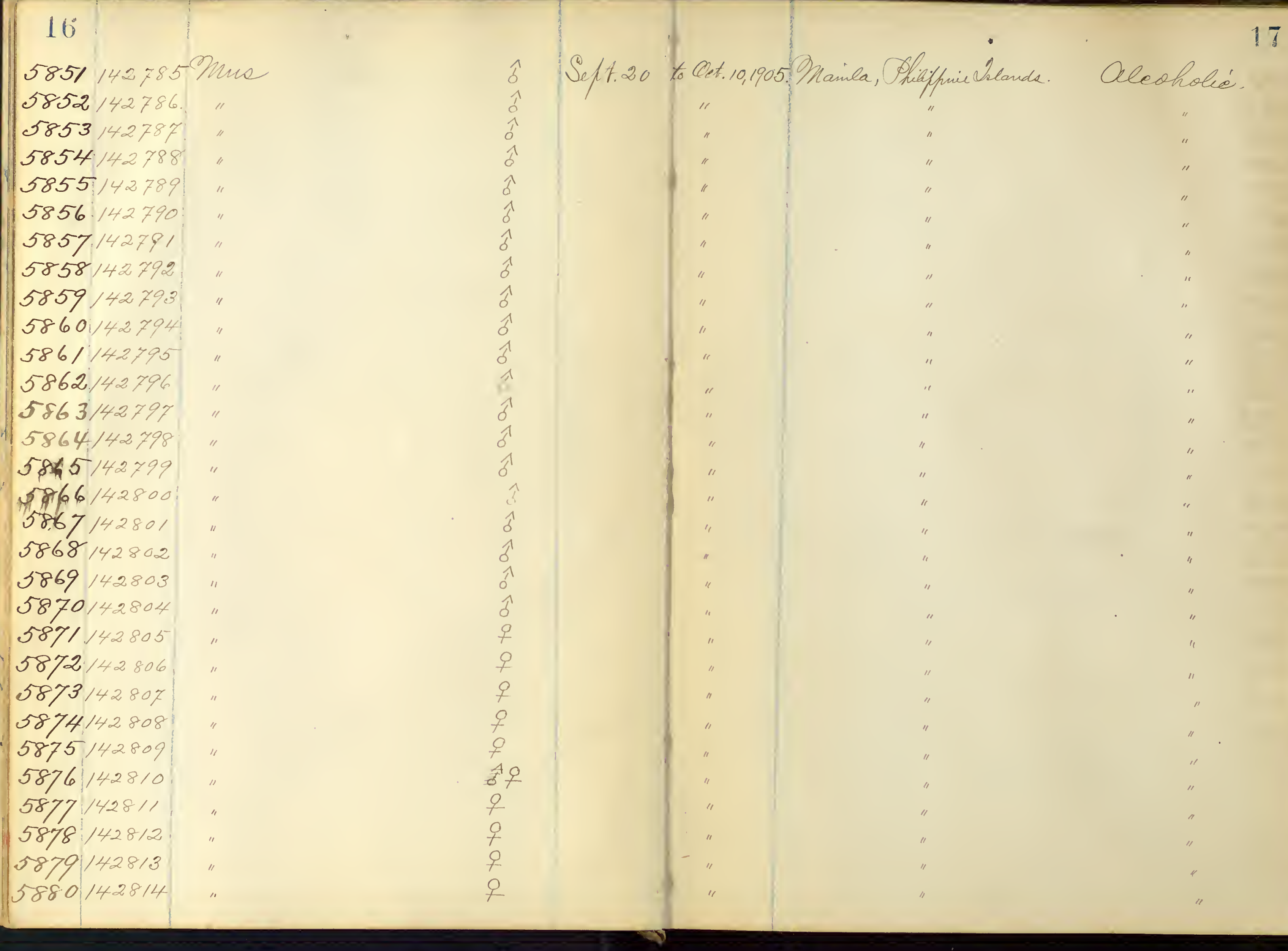




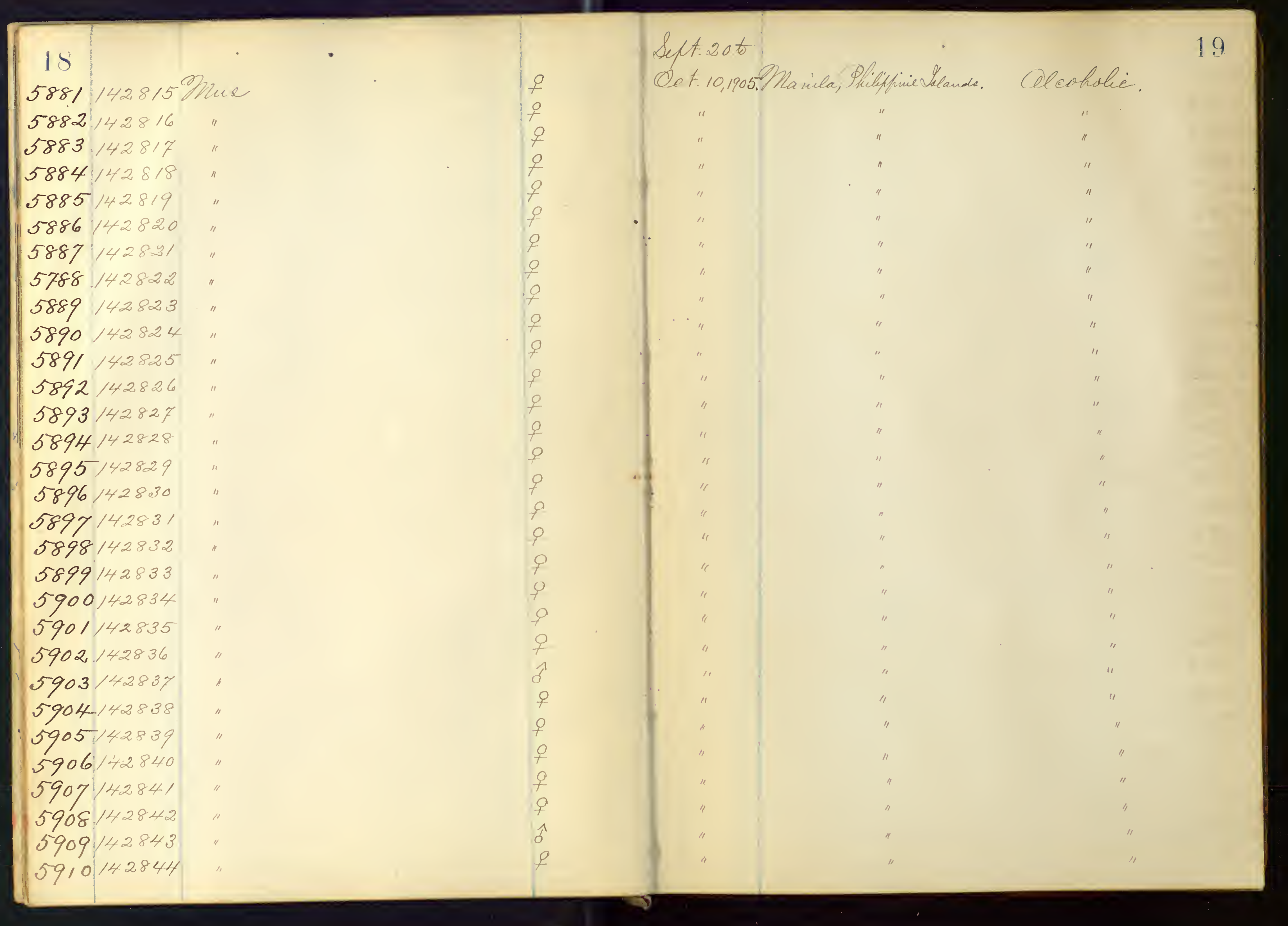




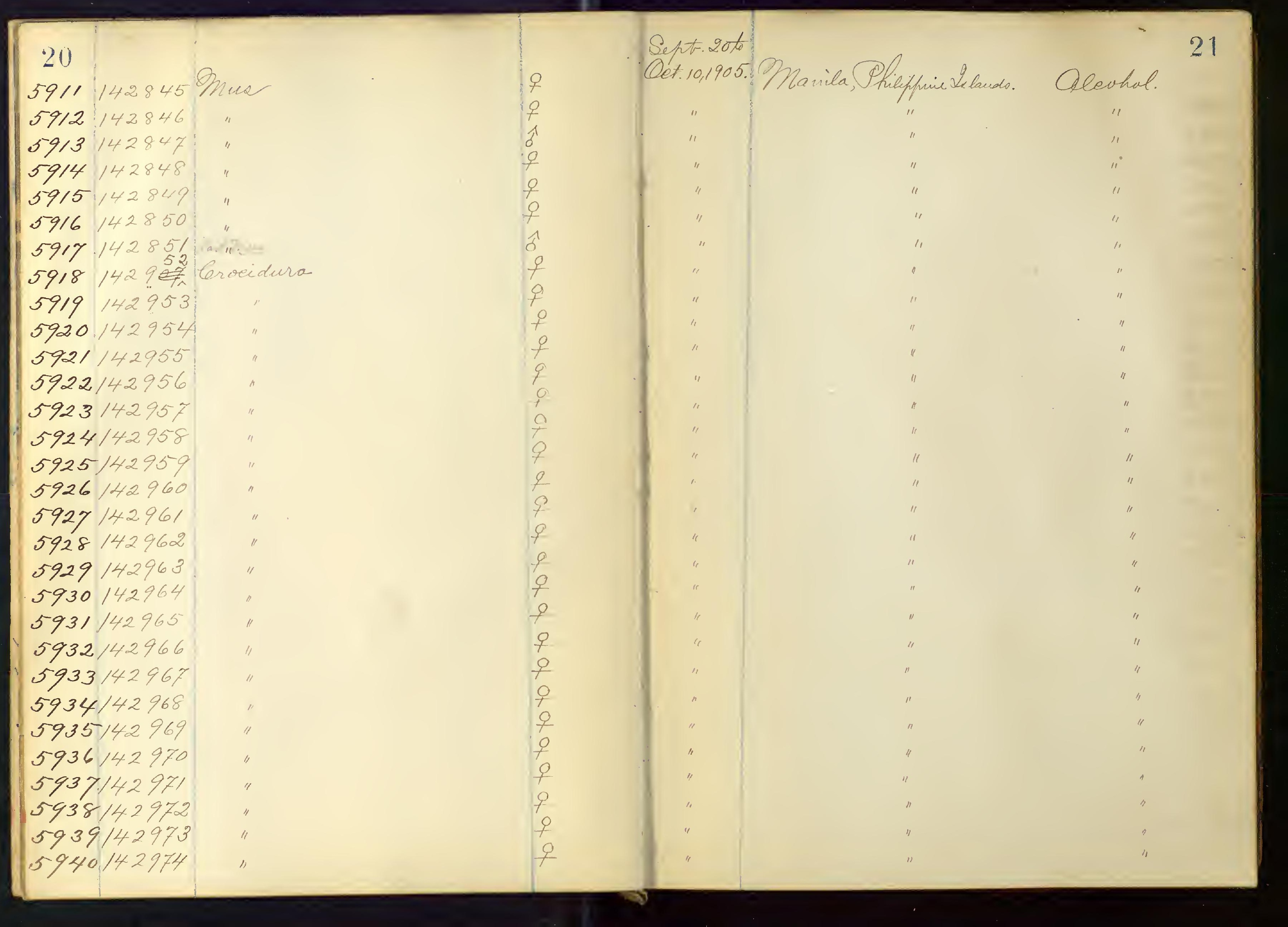




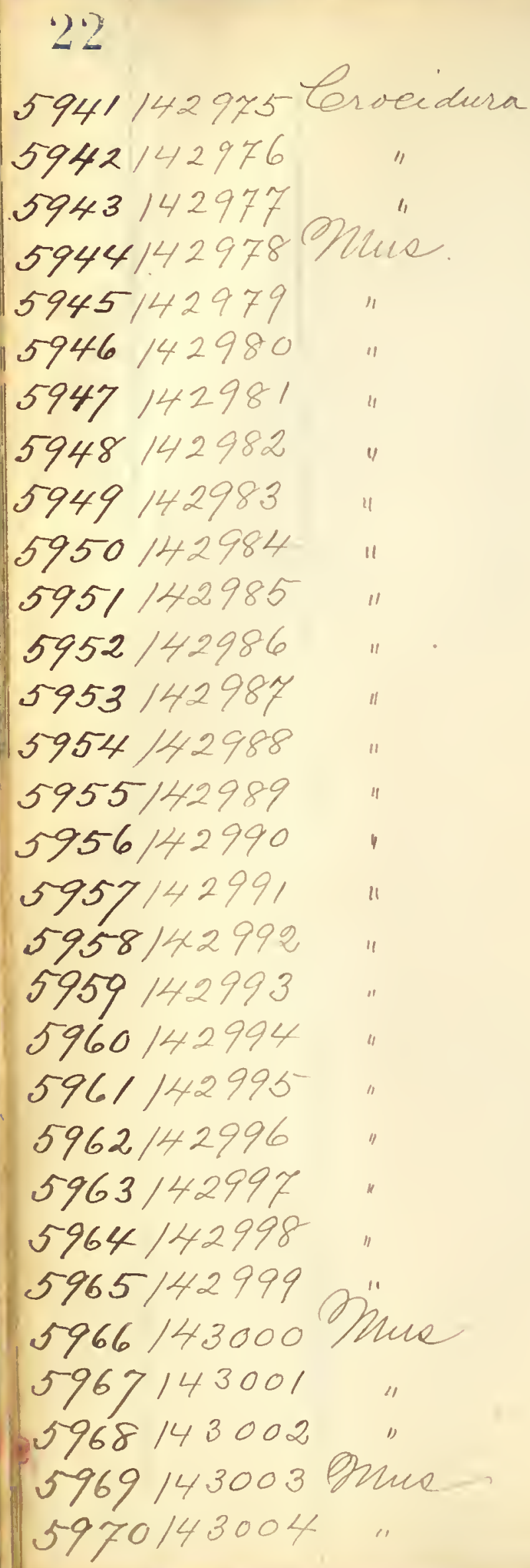




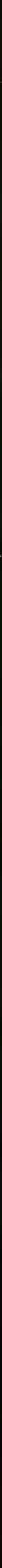




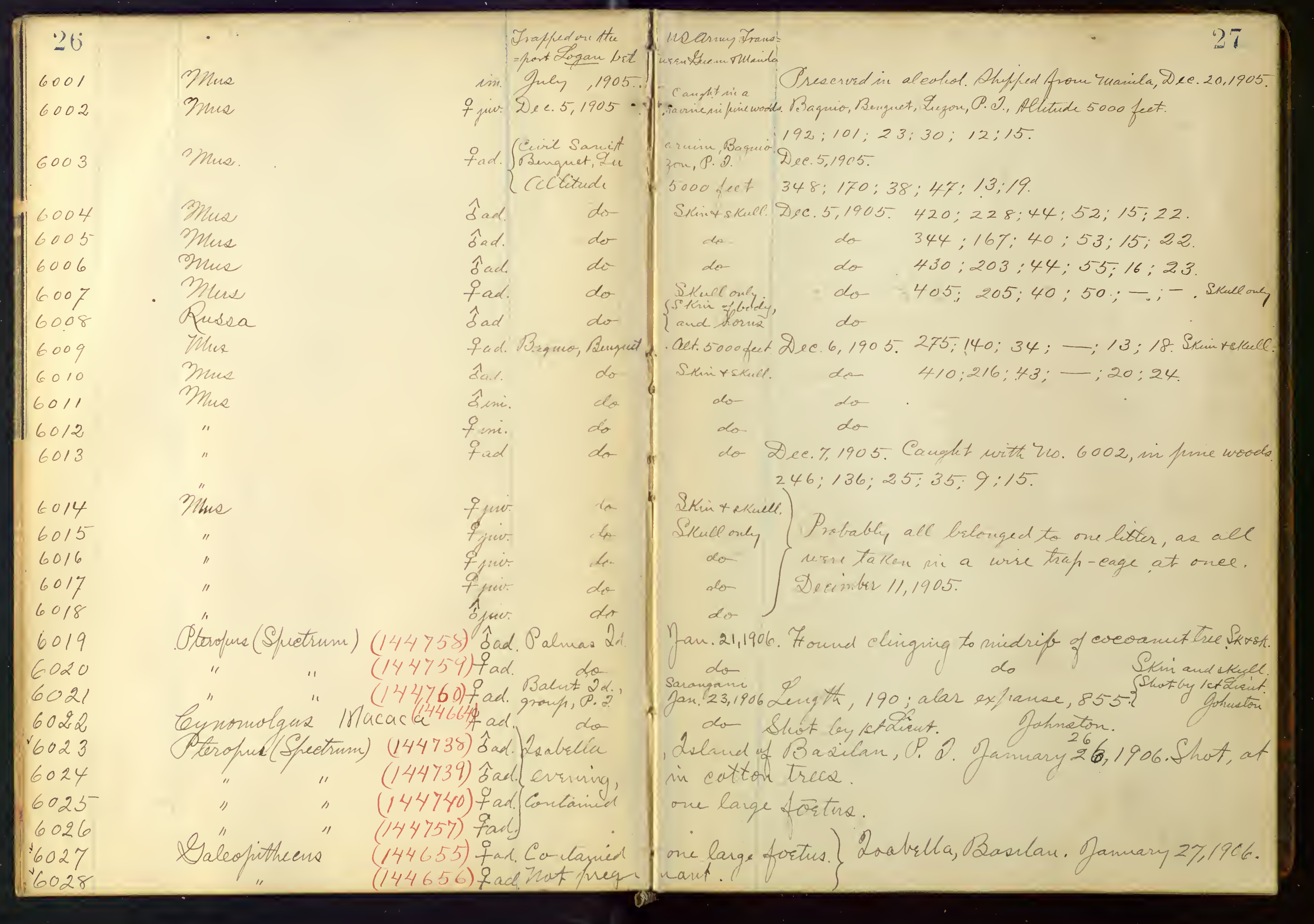




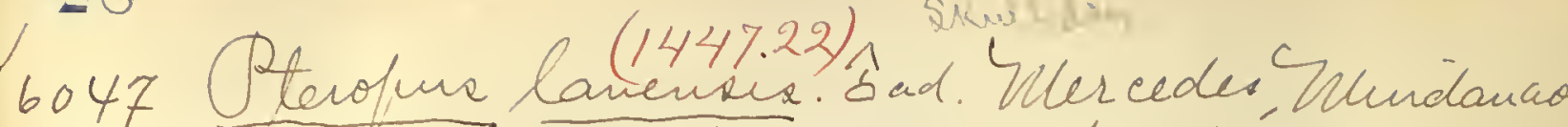
1265; Congect finger, 342; hid foot, 61 to end of hind livib, 440 Shot by Cafitani

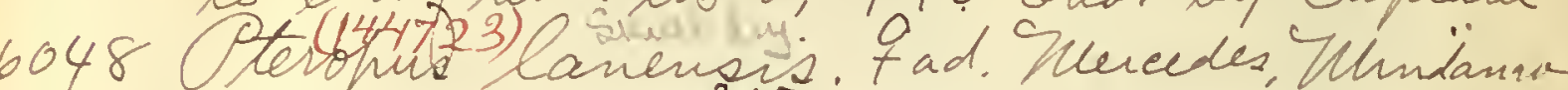
longest ginger ; hid foot. 58. head, end of trial livile, 430. Bleot by Oaftain Tamboanga, Muidanio, P. 0.

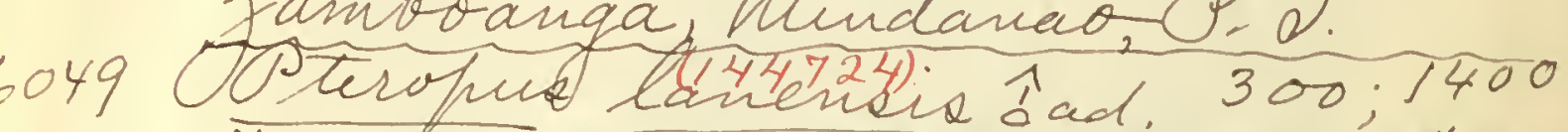

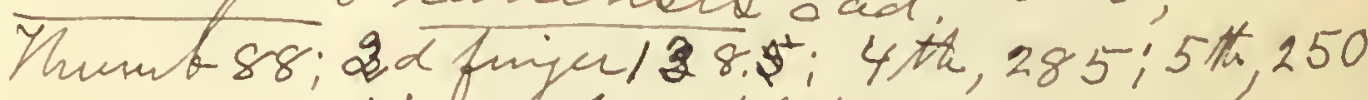
2.2; 2 nd fingu, elaw of (chord), 6; claws Ins hazet Bare porto and' Clavs blaek. Sheetrum $\quad(144741)$ àad. 210;910 4 th 117 ; 5 it 162 ; hind foot, 46; head, moutt, 27 ; to aye, 26; und hined linib 330 Bis dark hazel. Bare fearto blaek. Clans Samboanga, Mindanao, o. .

$605 \%$ Ccerodon $(144774)$ tad. 355 $390 ; 4$ th, 300;5th, 256; claw of Claws of hind linbss /st (inver), 20; 2net head, 94 : mose to uje, 36 ; nose, to

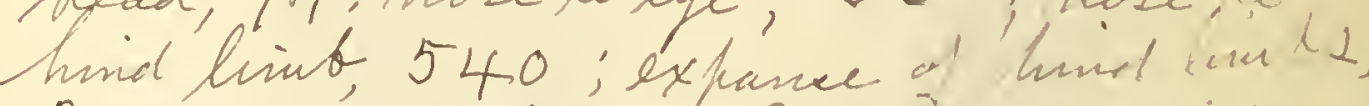
Zis yellowisl. brown. Bare prarto exeiht black; claus black, faintty dorn color on

6052 Ear abtor eroun, 35, eas abor notelo, 40', di=

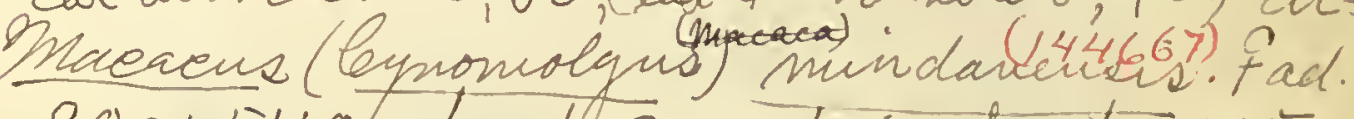
900:540; head, 97 ; hind foot, 15

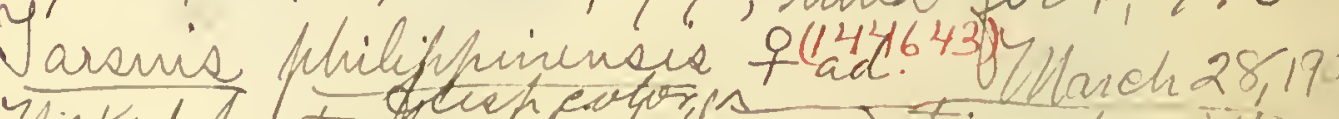

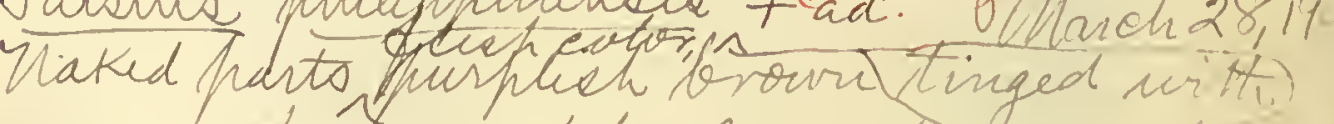
3.5 idrom noteh, 30 ; deaucter of cise. In anmae 3 paris, filled with milk
P.). Ylarch 17,1906. Length, Heorge Langhorme, II Eav., A.D. C., Usarmit. P.c. Wureh 17,1906. Length, 290; alar expanse, 1230 75; ear croure, 39 i car notoh, 39 ; lugth to Poore, 6tt Infty, Motruny,

March21,1906

$367 ; 62 ; 83 ; 40 ; 40 ; 500$

ifread of hind lumbs, 425, 8; claw titurb-(cturd), hind foot (mines to outer), 18, 20,20,18,17 ofijers 1st(tturd) 5y; 2 ned 90; turid (longest), 232 65; car crown, 26; noteh, 26; nose to angle of ontopread hind lunbs, $275^{-}$; derueter eye, 8. I bleck, fainteltipted with horn color.

\section{mareh 23,1906}

1545 ; firiqus: 1(thunt). $80 ; 2$ nd, $140 ; 3$ nd (longesdy thrmb, 25; 2 nd finger, 6,5; hind claws. 21 ; 3nd, 21; 4th, 21; 5tth, 19.5; hind foot, 63; argle of mouth, 36; mose to end of autseretelued 465 . Contanied one fortus (in formalin). unigs, black, ings prayish from motted witt edger and bifh. Sillot by Leonard Wood, ir ancter of eye, 12.

Iris yillowish boum; ear croms, 28; ear noteh, 36 Zautoanga, Minianao, P. .: Lis yellowish forom 4 or: 268; 274 ; 1.6., 68; head, 43; car from crour,

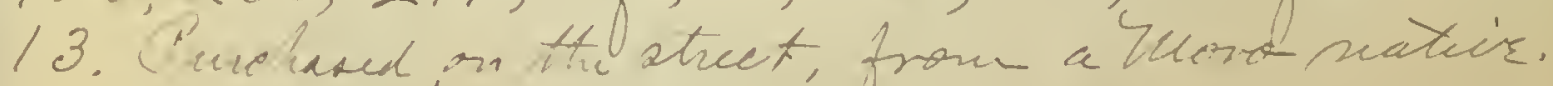


30 Prerceder, 9 miles ent of 2 anboranga. $6054 \sqrt{2 a t}$ (144812) q ad.

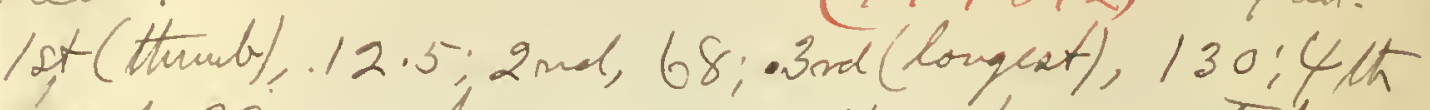
thead, 28 ; car from eroun, 14 ; from noteh 22 one large forstus frecerved in aleshal Stim

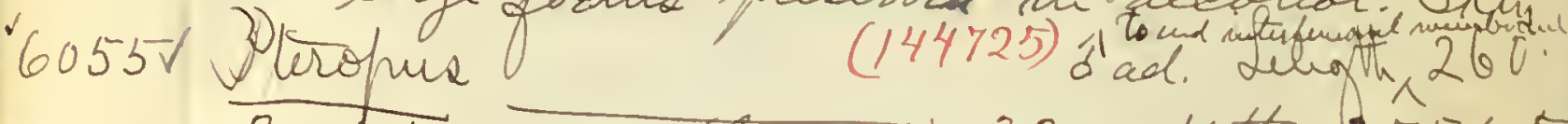
$2 m a, 130 ; 3 m($ longent); $330 ; 4$ th, 255; Aoréfinger (2nde, 6:5); hind doot, 57

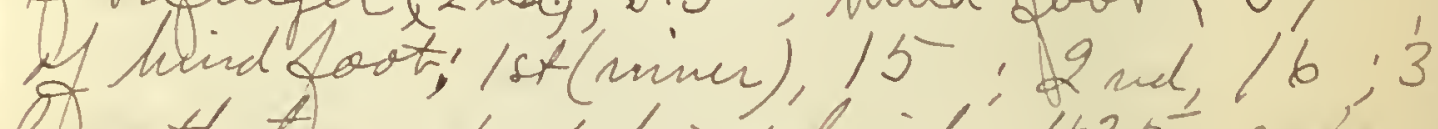

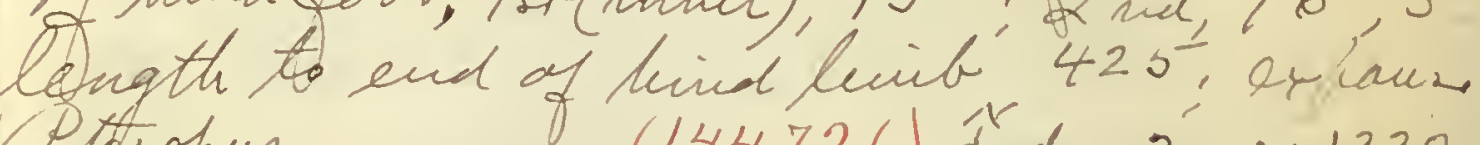
$6056 \sqrt{0}$ Peropus (144726) sod. 300;1330 $\frac{15 ; 17 ; 16.5 ; 16 ; 16 ; 12 ; 460 ; 410 .}{15}$ 605 F P Peropues (144727) Fad. 320;1320 $15 ; 16 ; 17 ; 16,5 ; 16 ; 460 ; 410$.

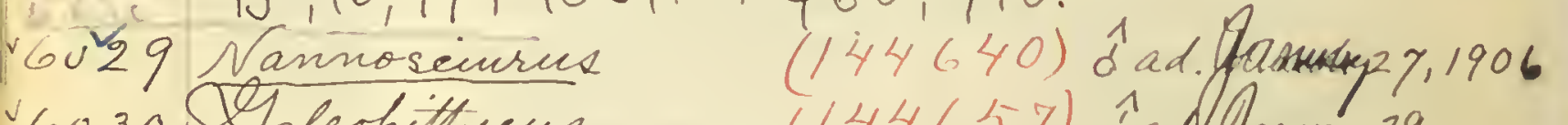

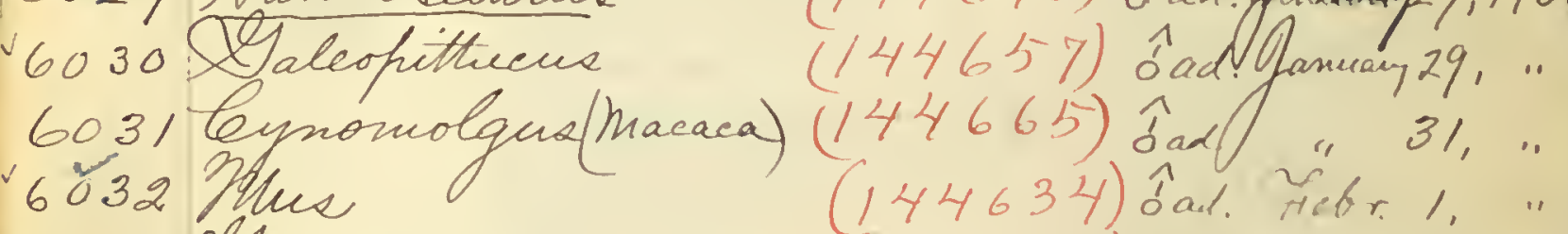
$\checkmark 6033$ Naleofitterens

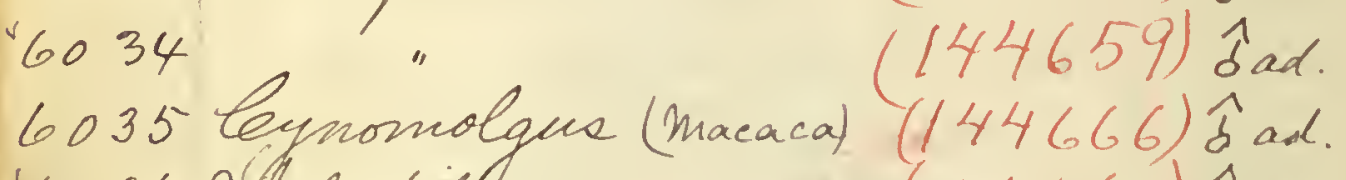
16036 Salespittecus 6037 6038 6039 Whes 60340

6041 Holespitterens

6042

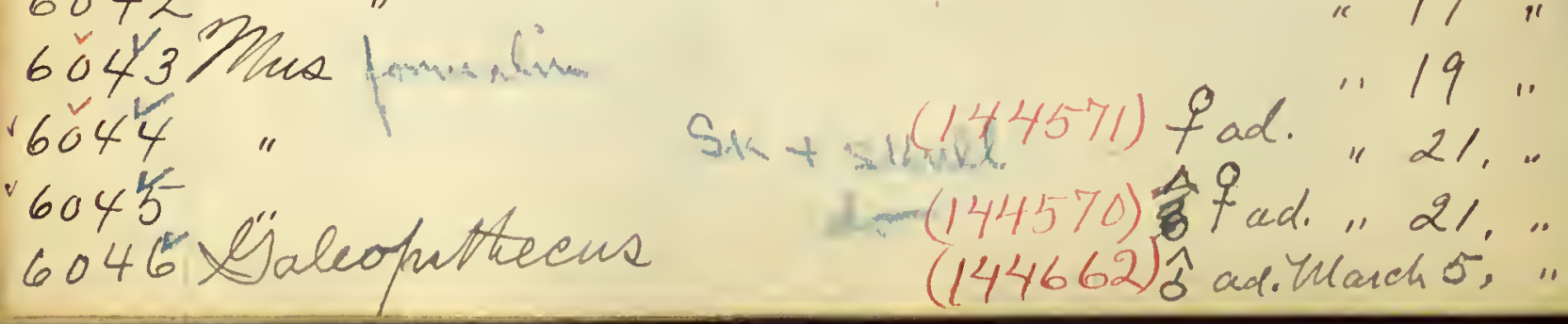

Wuach $28,1906$.

31

120; atar expanse, 500; candal vert 1, 25; fingess.

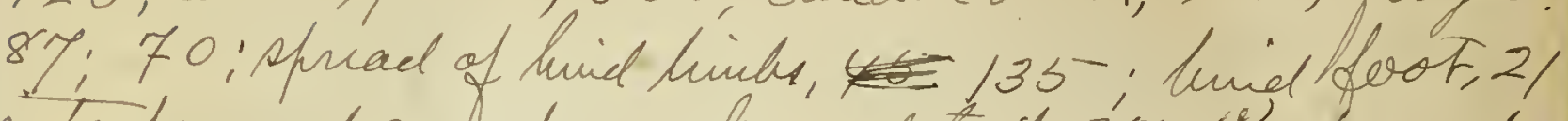
interfenoral nofubrame beyond tail, 2\%, 4500 y and and skell.

alar expanse, 1312 ifnigers: Ist (thunit), 75; th, 223 . Claw of thiub (chord), 19 ; claw of hiad, 77 ; ear leroun, 42 ; noleh, 39 ; Clawts

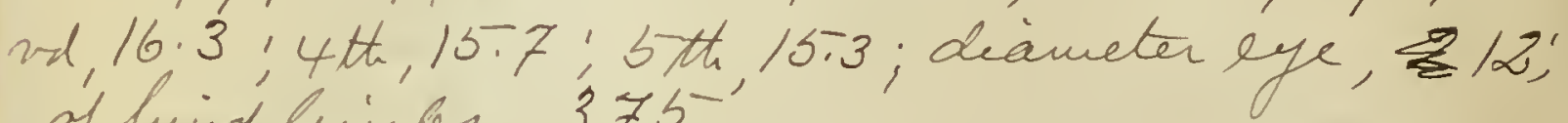
of hind livber, 375 :

$80 ; 140 ; 340 ; 250 ; 235 ; 18.5 ; 6.2 ; 60 ; 78 ; 41 ; 41 ;$

$78 ; 140 ; 336 ; 260 ; 233 ; 19 ; 6 ; 65 ; 78 ; 45 ; 43,5 ;$

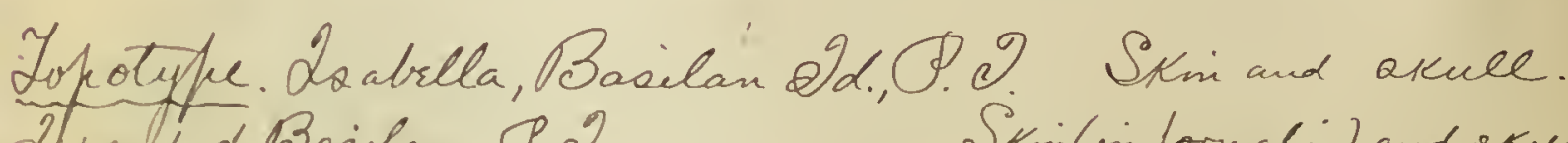
Strin (nifformatiin) and exwell. Skin and stuele. dow do

do.

dow

do

de

che-

do

do Shuce, the there y I liued

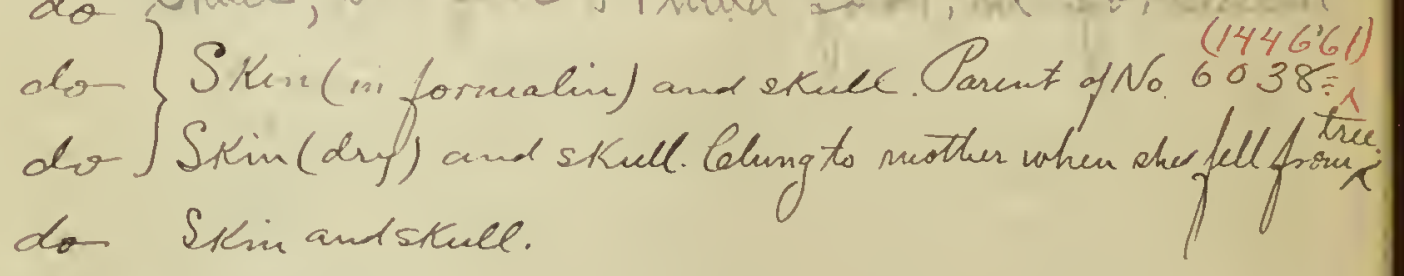

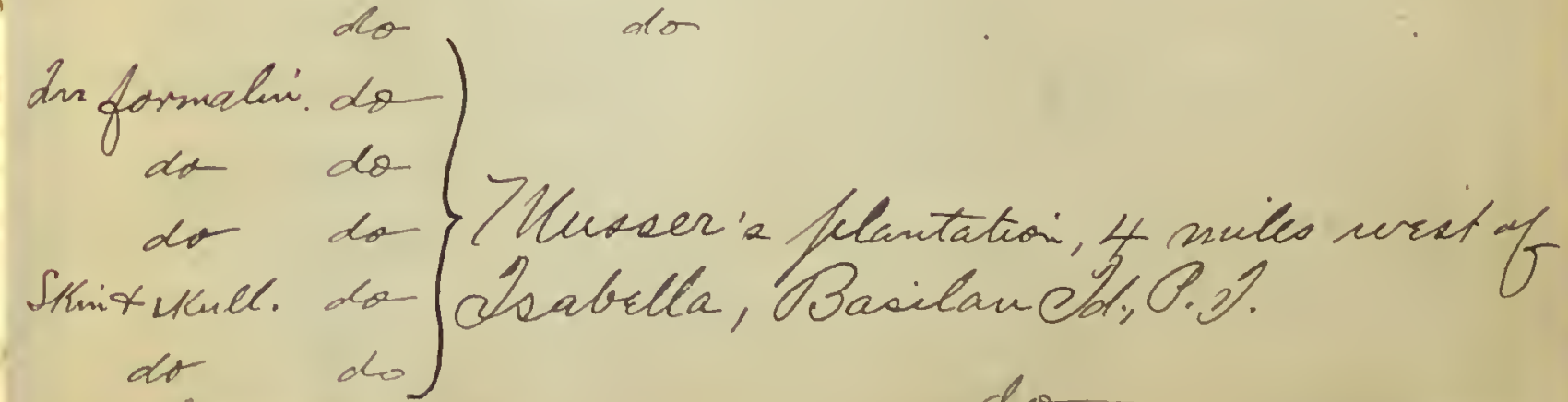


32

6058. Acerodon

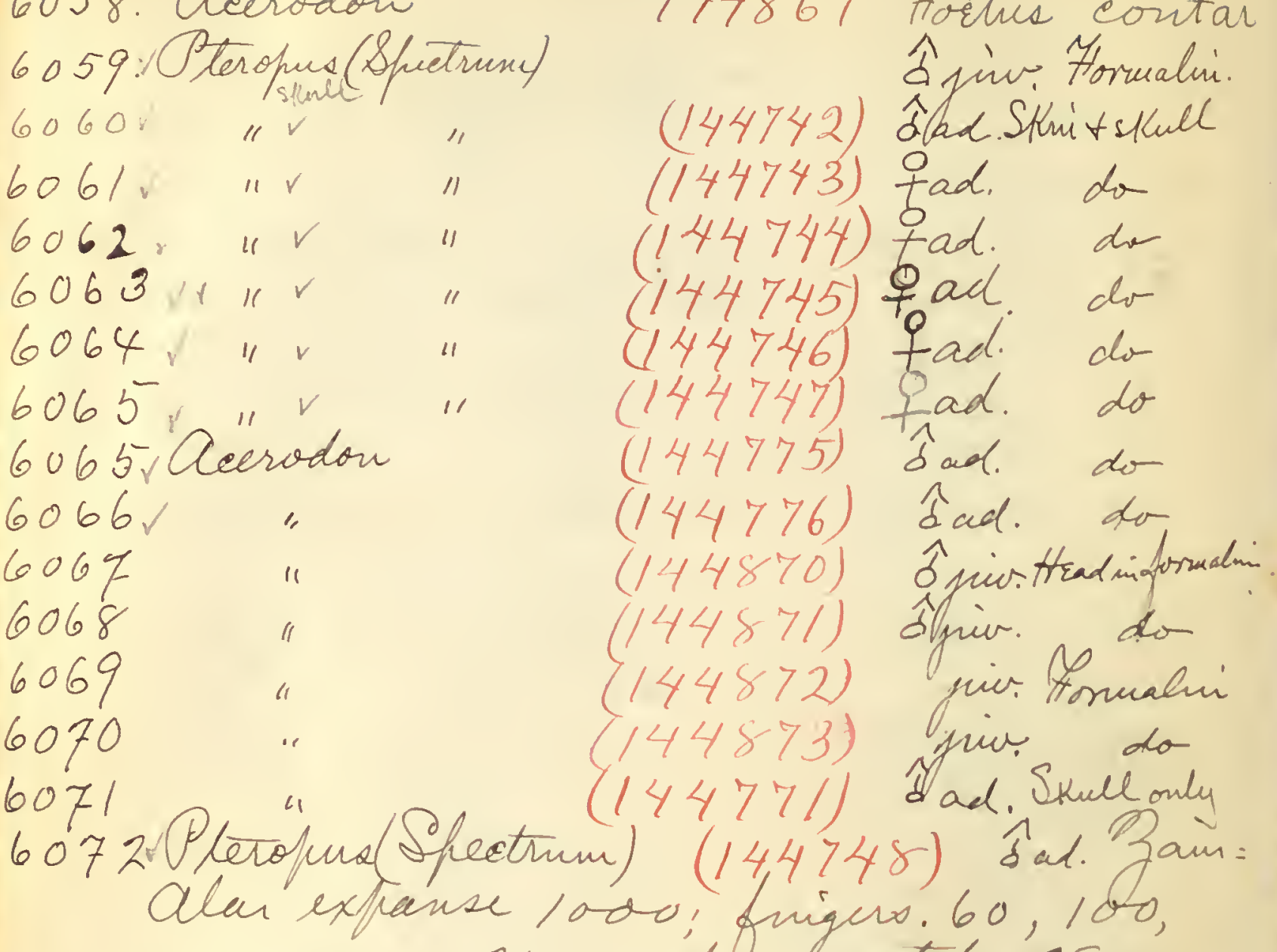

Alar extranse 1000; finigero. 60, 100,

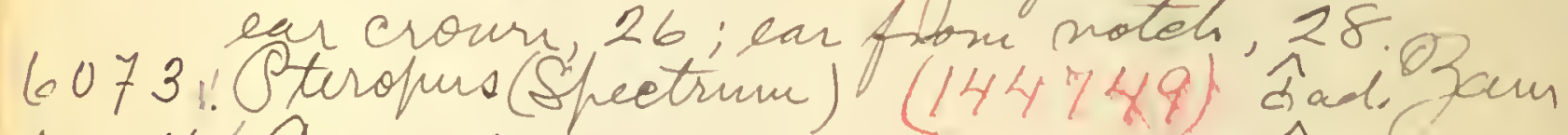
6074 . Accrodon

6075. Pleropus maceoyi. (144728) Aad.
6076 . Acerodon mad.leabo

hase to angle of month, 38; eyc, 38;

hind link, 560; ear above croun, 38

foot, 78 ; frigis: 1st (thunb), 95; 137;

thumb, '26: Claw of gorrafinger, 8.5.

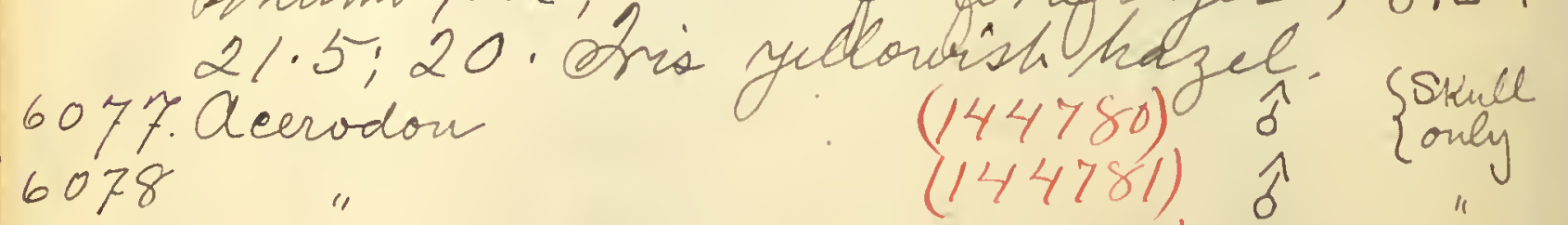

6079

$(144782) \hat{~}$
$(144783) \hat{\sigma}$ ned in cNo.605\%.(144774)

33 Malanifa 2iland, off D. Unudarao, April 1,1906.

da do

da

do

do

do

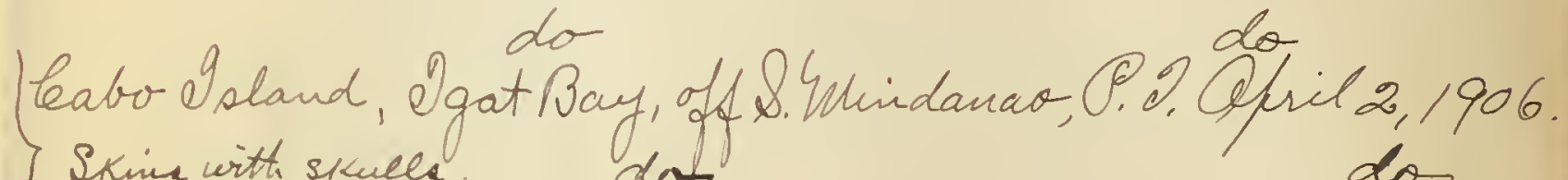

S Skinewith skuela. do do

do

do

do

da

da

do

bormpa, Inidanao, P. P.? April 5, 1906. Length, 210. 260,200,175; 'hind foot 5'2, head, 69;

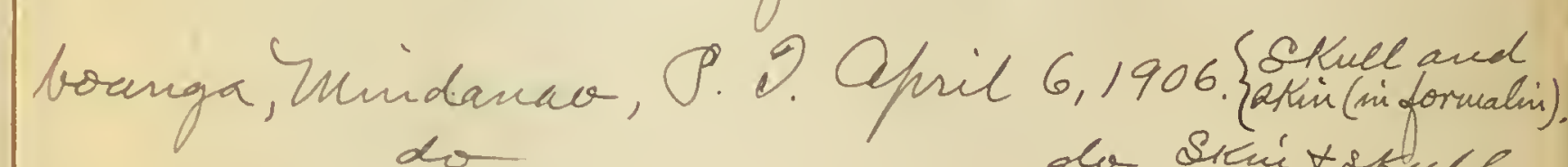
do

2el, Ggat Bay. Apil 2, 1906. Dugth, 400, ear 83; ocieiput, 100; end of aulshetolued abour noteh, 37; alar expduse, 1545; hind 3d(lougent), 403; 4to, 320; Sth, 275; clave of Anid dawa (iniver to outer): 19, 22, 21, 22, Wing, leg, and skull, in alcohol.

leaboliland, Ogat Bay, off \&. Mundanar. Afril 2, 1906.

do 


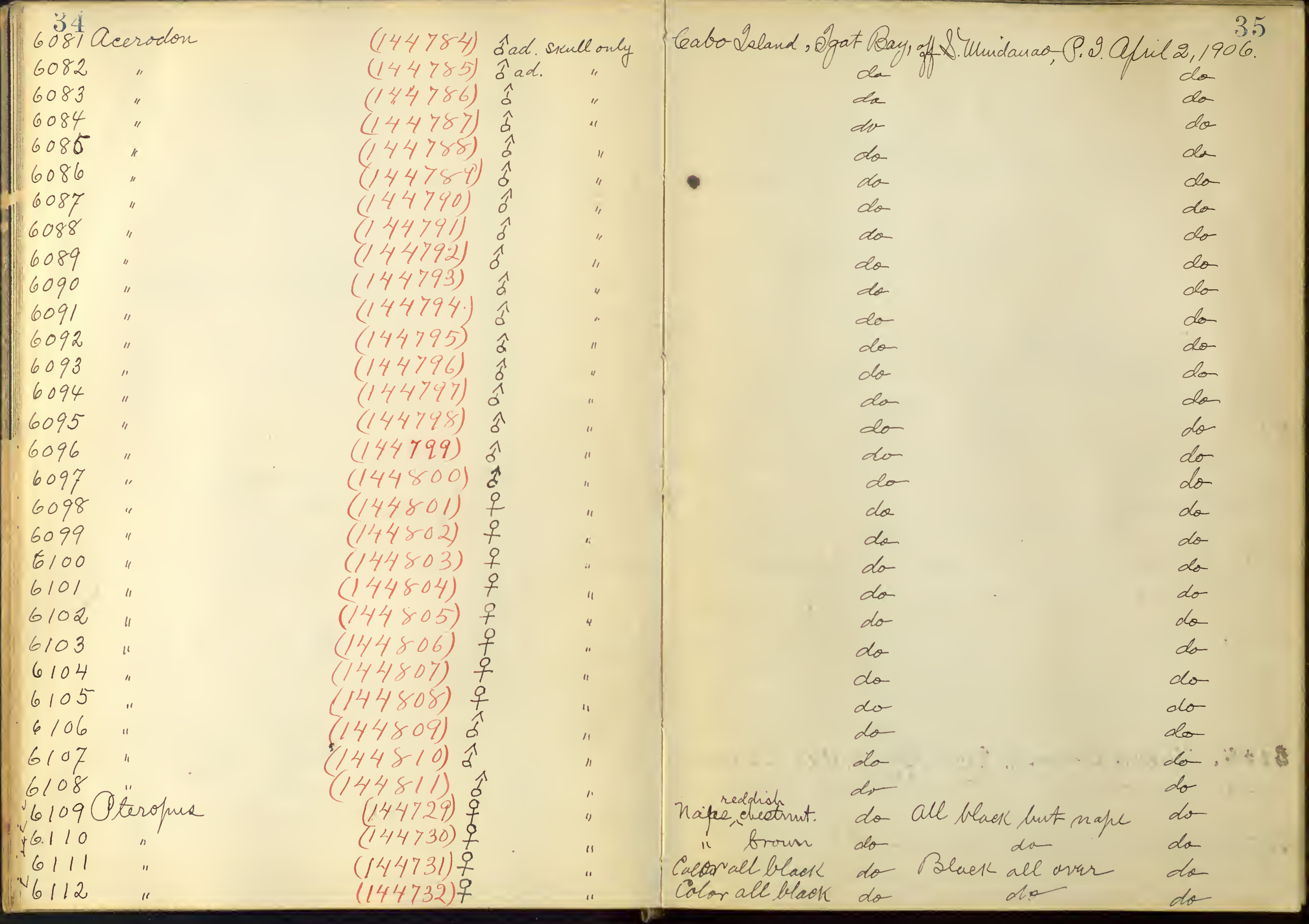




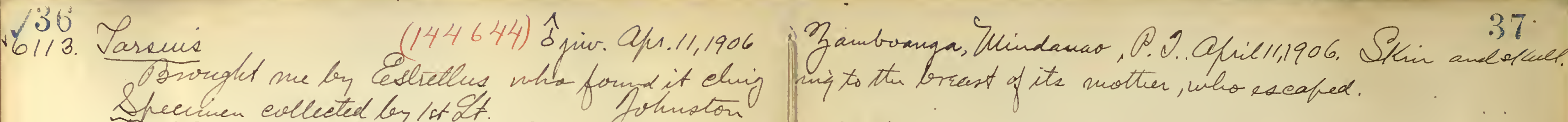

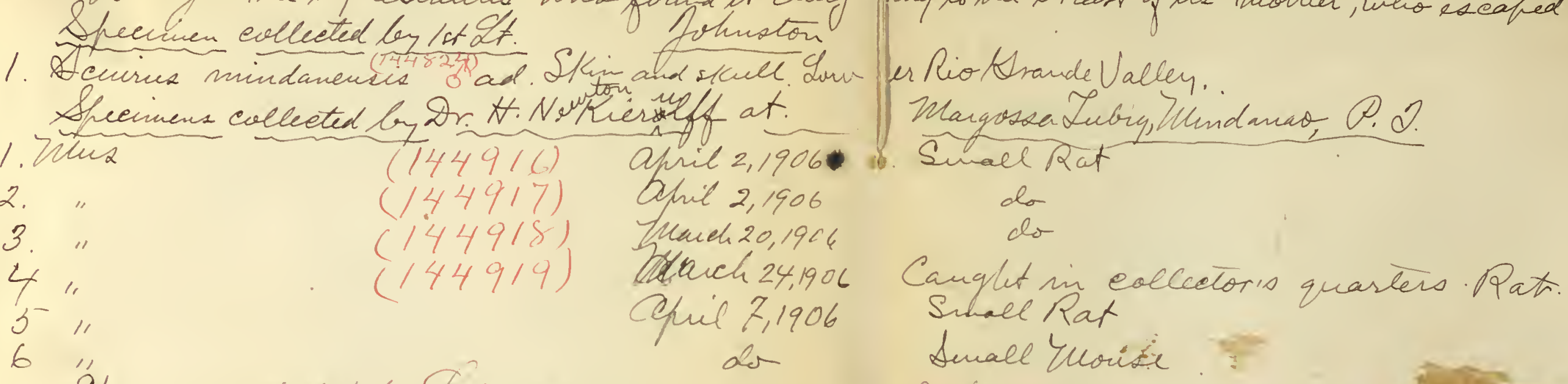

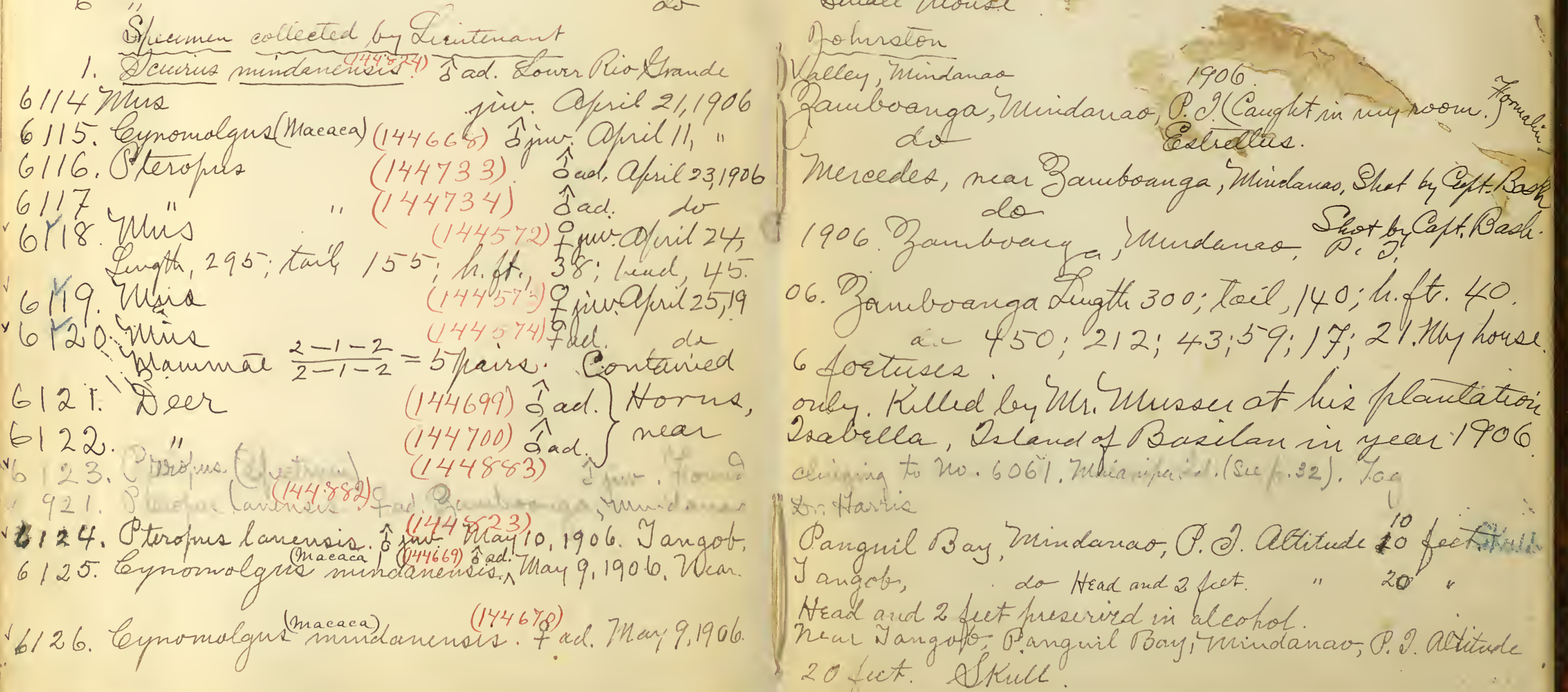


35

39

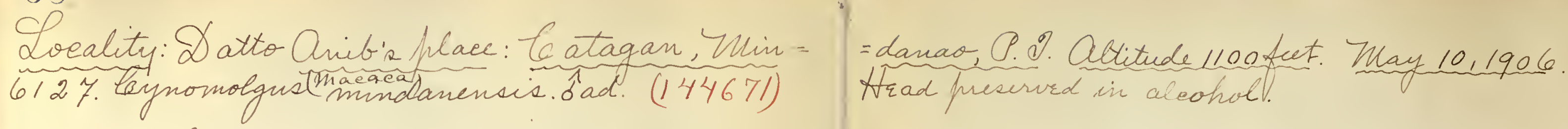

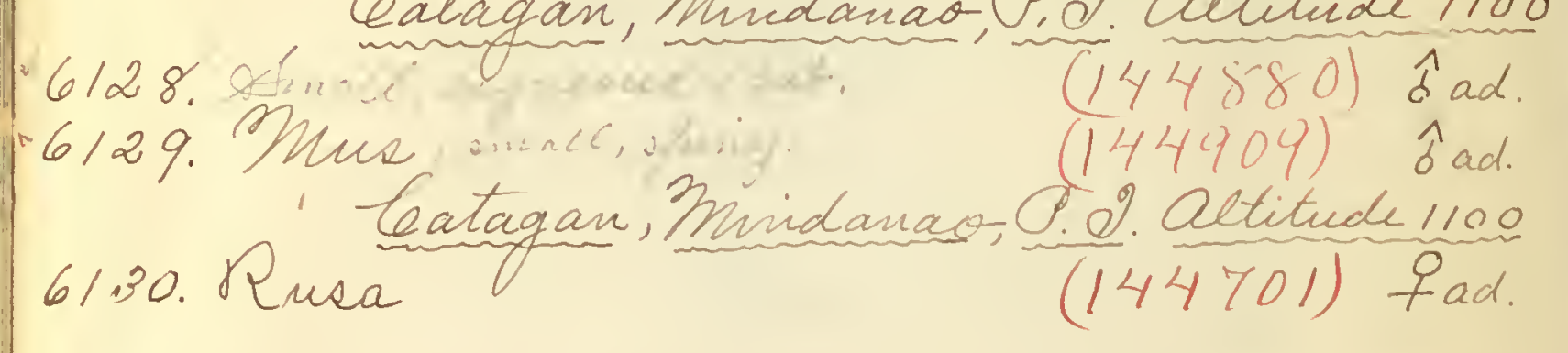

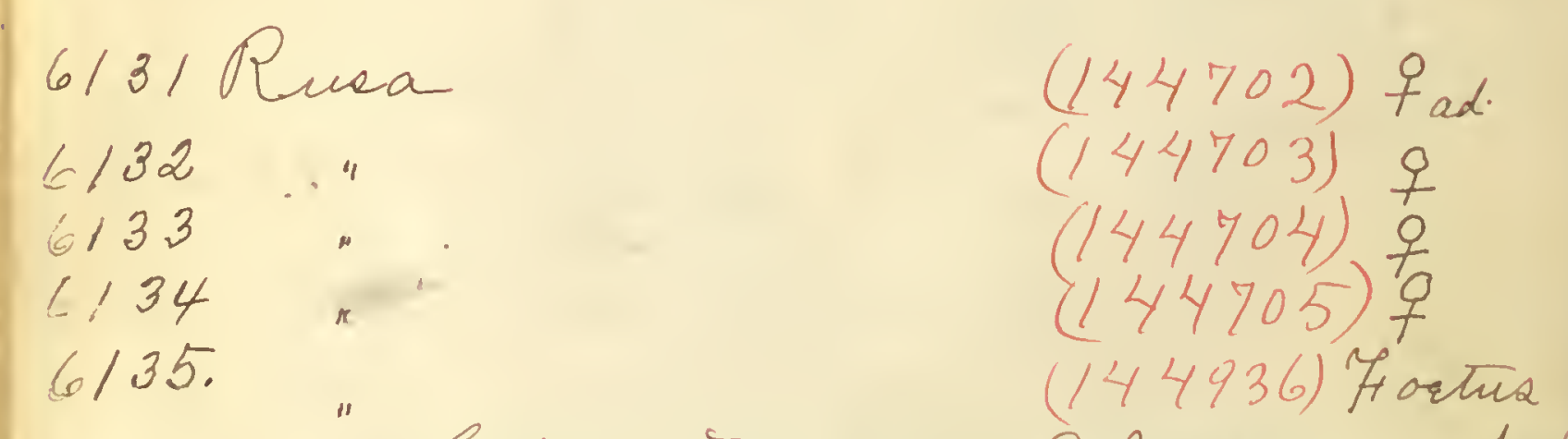

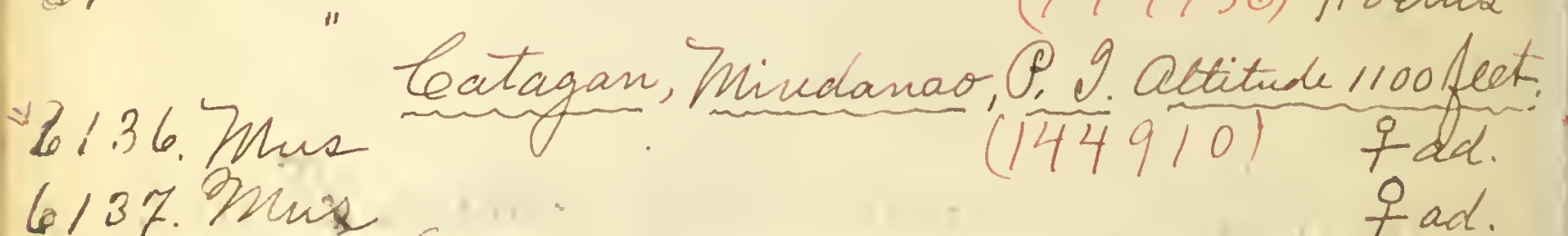

Tut May II, $19 a 6$.

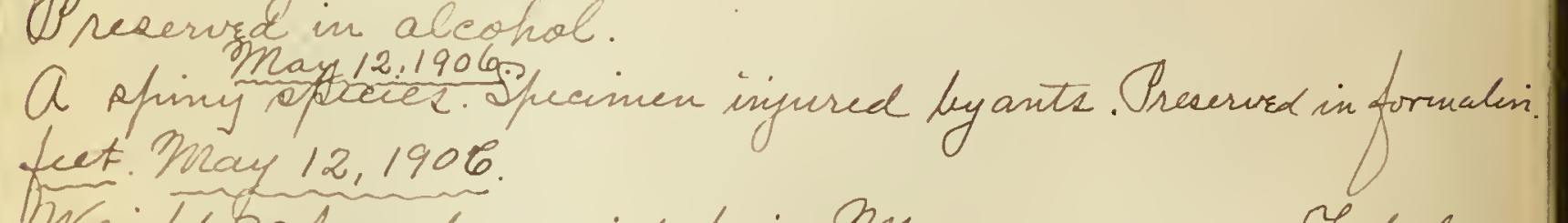
Weight, 90 foundo avoridufoir. Measurementa: Lotal Cength (otraight), 1620 millimeters; girtt of chest, 850; of belly, 1120 ; girte \& bins, 910; of thinnest, fart of heck, 288: ingth of head, 300; forefot, 270 ; hindfoot, 360 ; tail vertebrae, 150; tail to end of hairs, 240, ear above crown, 130; aborr noteh at base of opening, 110; brad ras fiad to dugle of moutth, 90; to eye, 160; to base offear, 245; to tif of car, 390 ; from head of humerus to Dhead of femur, 660: height of animal at shoulder, F80; hight of anisual at hife, stso. Skin with steull.

Young of ho. 6130 (14478) reserved in formalin. A smalebat. Preserved in formalin. May $15,1906$.

Precirrd in formalin. A male Rat. 1300 fect. Mnay 15.1906.

Subano nasne: Kahl-Lagh-chí-e. 

4U Catagan, Mindanav, P.Q. Allitude 1100 feet
6140. Pufta 6141. Muna 6142

$6 / 43$

6144. Rusa

6145. Paleopithecus

6146. Rusa

6147. Mne $6 / 48$

6149. Shis

to 150. Viwra togoluma

6150. Rusa

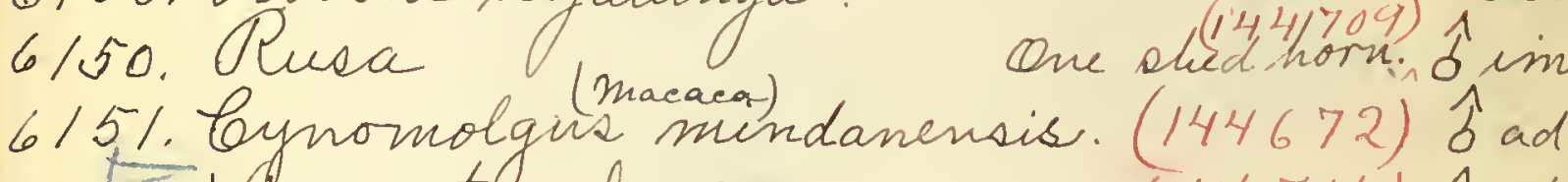

$6 / 52 . V$ verra tagalunga.
Subano name: Loó-bing.
Hiay 16,1906.

Skin and lower jow.

teet. Bnay 1 , 1906.

41

ASutano name Joo'-boong. Preurved in formalin.

Mecauremente: Fotal length (atraight), 1465; girte of

do cheat, 850; of belly; 1050; of loin, 880; of thimelet rart of neek, 446; head, 365; fore foot, 240; hind 400t, 345; tail virtebrae, 145; tail to end of hairs, breadth of ear following eurve, 94 ; distance from frowt of mavald/had to: angle of mouthe, 100; Je, 160; to base of ear, 250; to tiff of ear, 380; he ad of humesur 790 : wove to base of hom, 245 hight at hif, 840; greairst length of hornkchord), 318; greatest brractte of

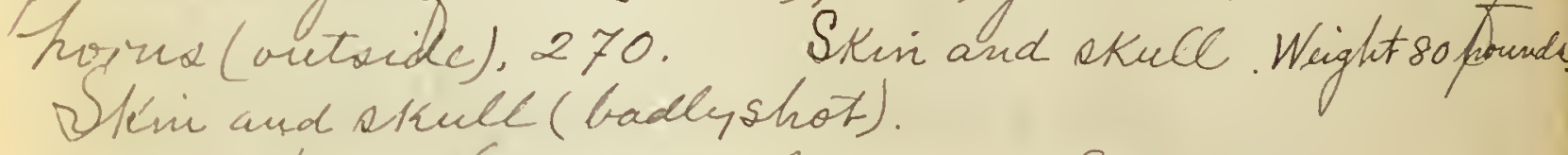

Home jrom Ceatagan. Hnom a Subano im deprualui: $223 ; 108 ; 27$. Qne lookte, Subanoname: Loó-bing. Sumnit of Mut. Bliss. Eletitede 5750 feet May 30,1906. leatagan (Datu Aricis place). "1100" "14," Length, 955; tail virtebrae, 350; hairs, 366; hind " foot, 84; head, 136; car erown, 38; car noteh, 40; nose to: angle of mouth, 65; eye 55; base of car, 111; tif of ear, 161: end of outstretched hind lisit, 890; diancter of rey, 14. 
12. Ceatagan (Datu Aritis fluce), Mindanao, 6/53. Oaradoxurus
Q.2. Actitude 1100 feet. OMay 20,1906.

Lugth, 870 ; tail vistebrac, 410; hairs, 440 ; hind folt, 78; head, 112; ear eroun, 38; notch, 38; mose to angle of mouth, 50 ; eye, 40 ; base of car, 90; tof of ear, 135, ind of outstretched hind litit, 635, diamecter

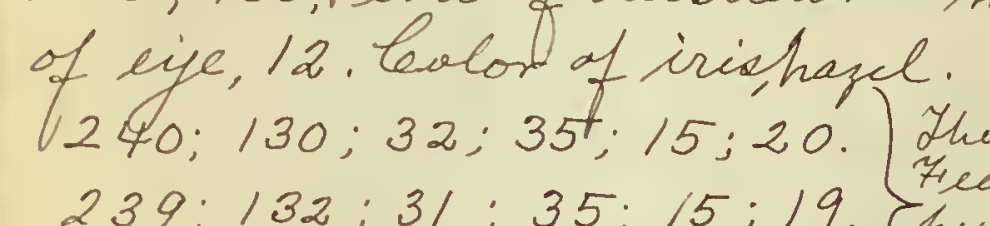

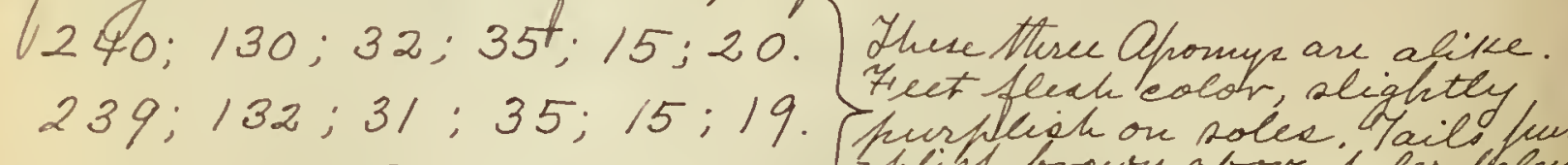

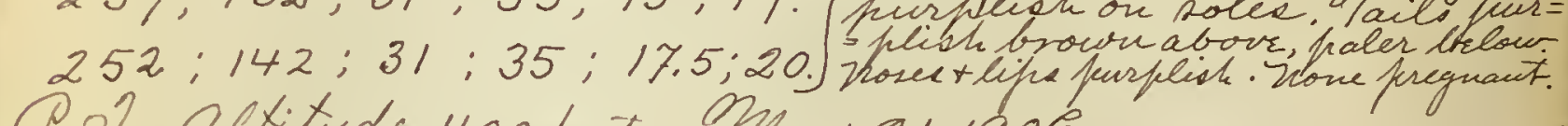
Q.2. Altitude 1100 fect. OMay 211906. 235; 127; 25.5: Sthin and arull. 210: 133: 45. Snie oefia. Vubanoname: Whh-maht'. Q. O. Altitude 1100 feet. Mray 22,1906. 1. Q. Werticude 1100 fut. May24,1906. ant-eatern. Prenerved in forme

Horne, only.

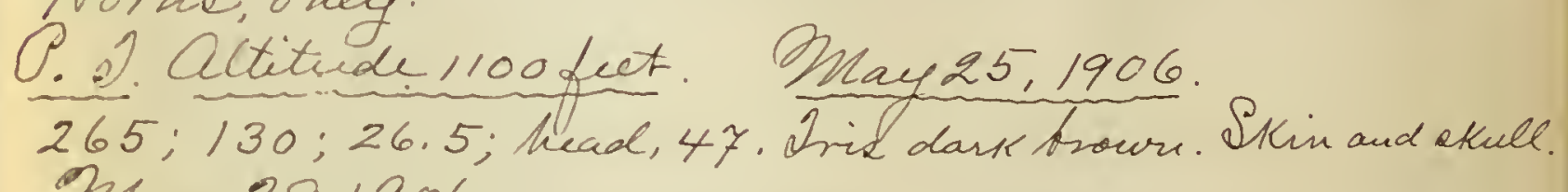
Mlay 28,1906 . $16 \% 64$. Dhponize 16165.

"6166. F" "6romys

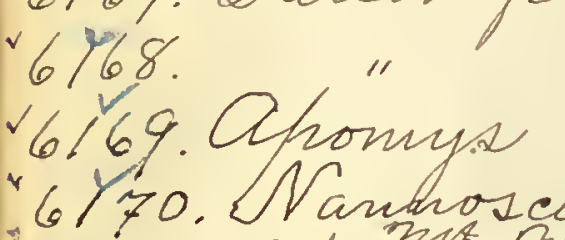
$248 ; 143 ; 31 ; 36 ; 15.5 ; 20$

2,$28 ; 134 ; 31 ; 36 ; 15 ; 19$

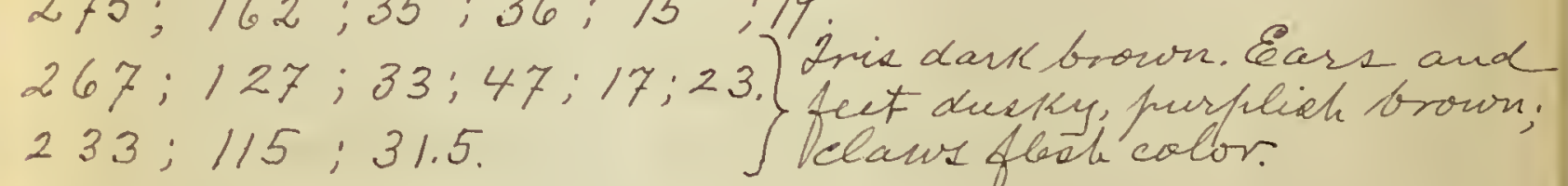
$270 ; 151 ; 31.5 ; 36 ; 15 ; 19$

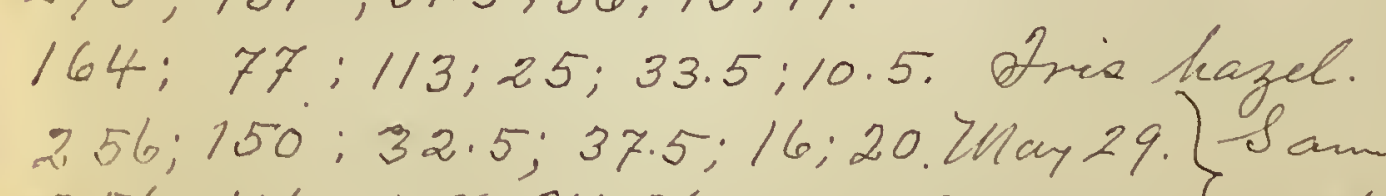

6 .

$16 / 72$. Aforment Bher may 3011906 .

$56 ; 156 ; 158 ; 34 ; 36.5 ; 17 ; 21 . " 30\}$

Same trape

6773 


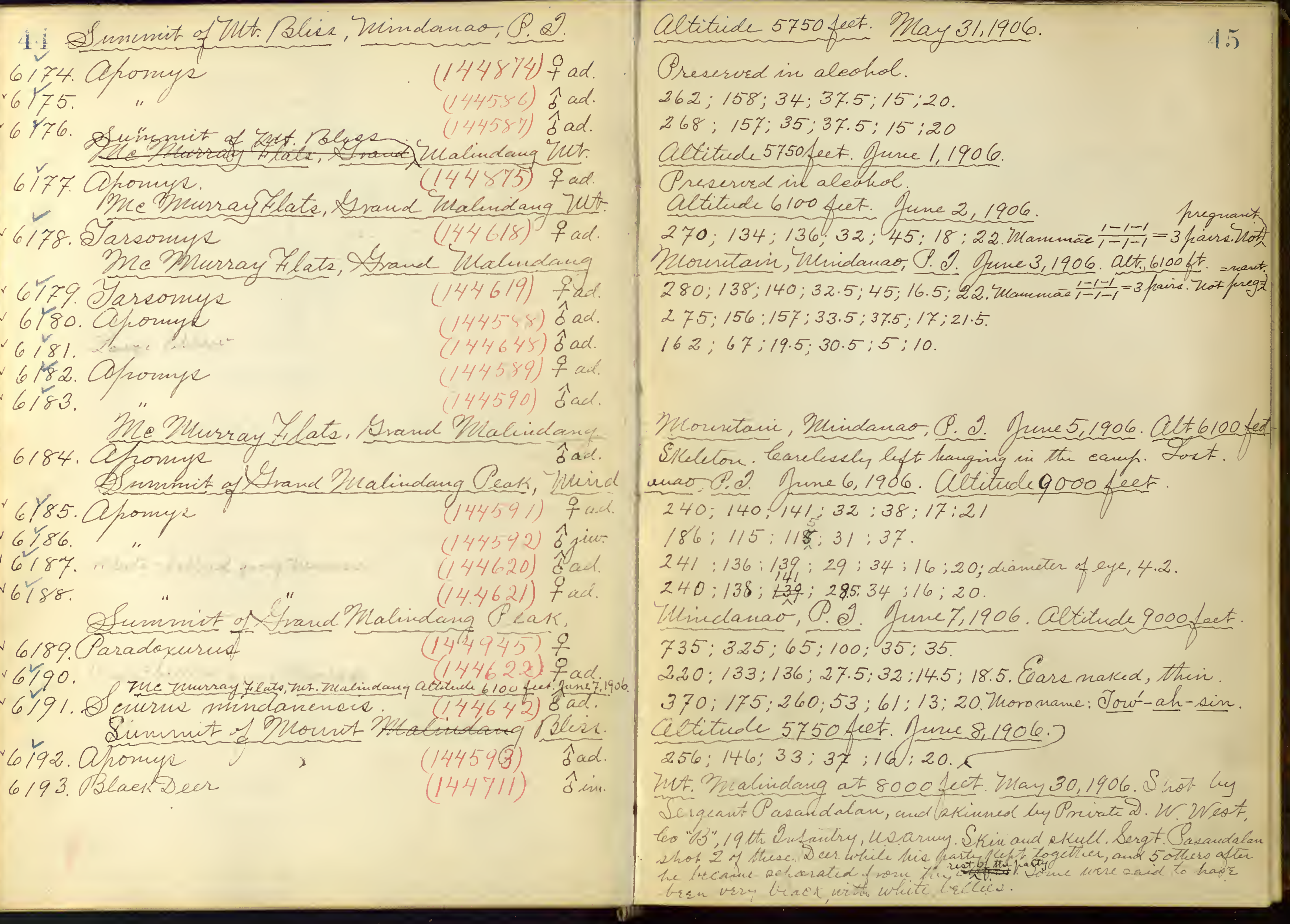




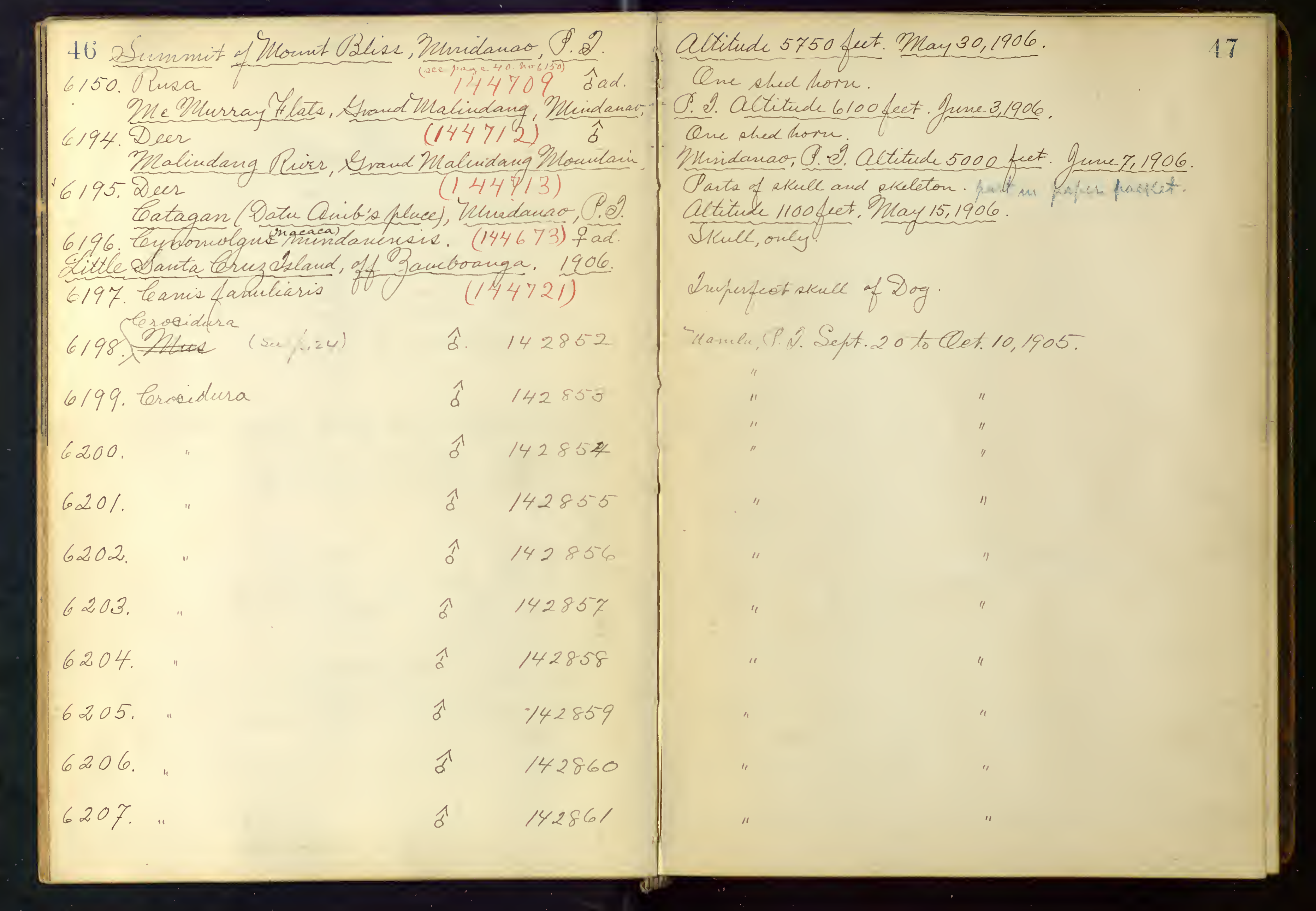




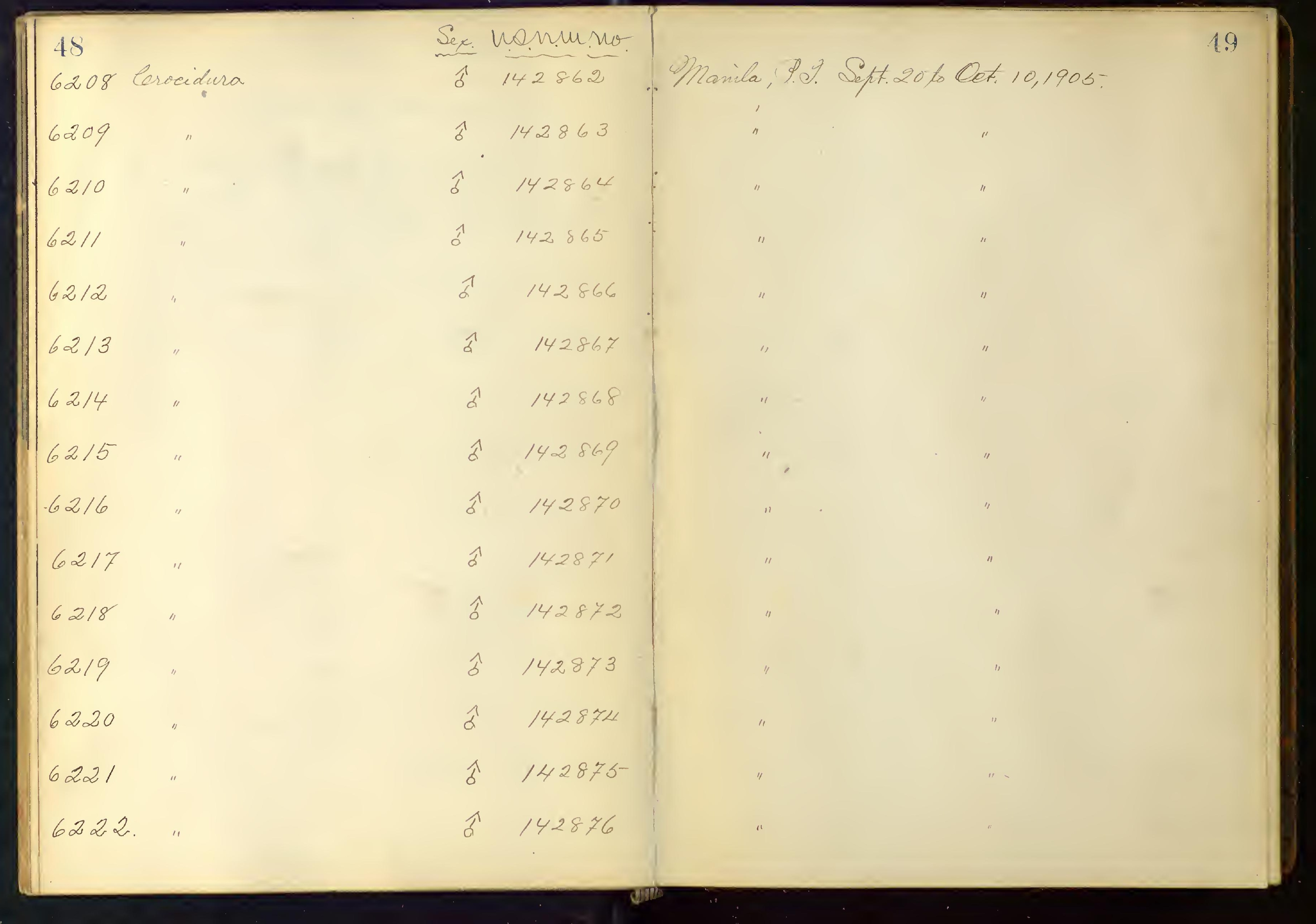




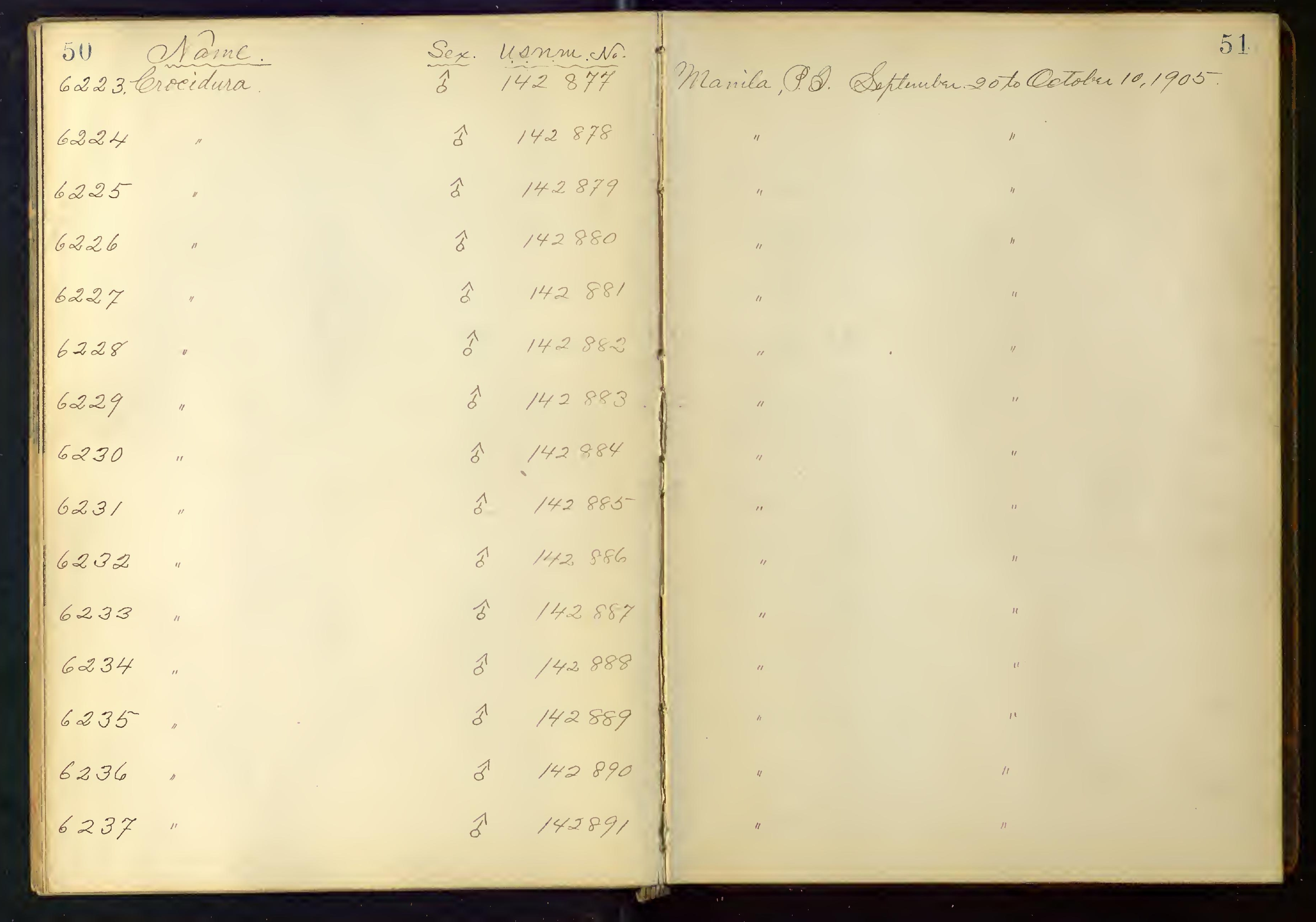


52

6238 Crocidura

6239

6240

6241

6242

6243

6244

6245

6246

6247

6248

6249

6250

6251

6252 $\frac{\text { Sex }}{\text { s. unne vo }}$

$\hat{d} \quad 142893$

ㅅ․ 142894

$\hat{\delta} \quad 142895$

$\hat{\delta} \quad 142896$

今. 142897

$\hat{o} \quad 142898$

के 142899

$\hat{0} \quad 142900$

त 142401

1 142902

1142903

$\hat{0} 142904$

a 142905

ले 142906

Manila, P. I. Sehtenbrr 20 to Cetobr 10,1905-

5.3 


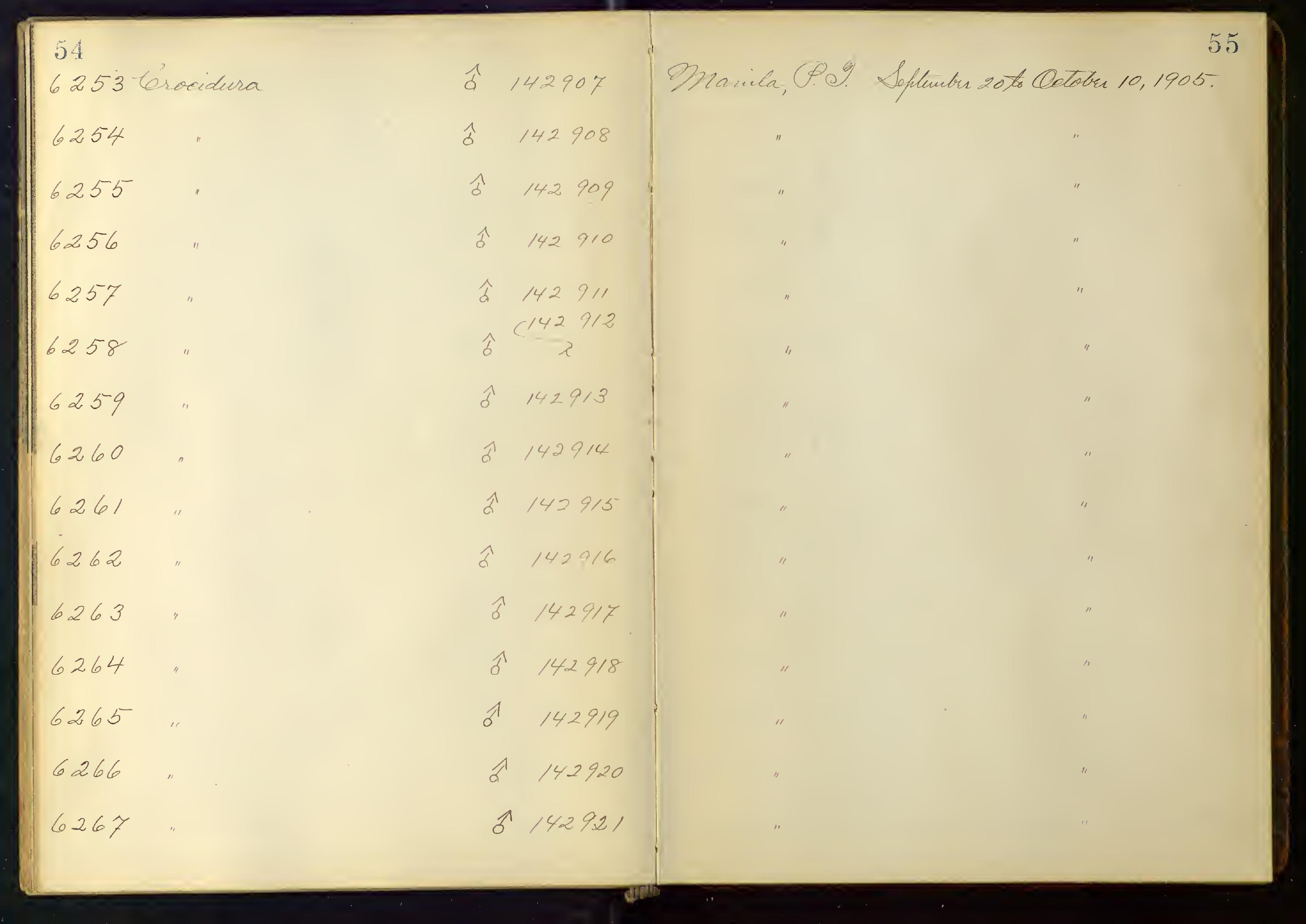




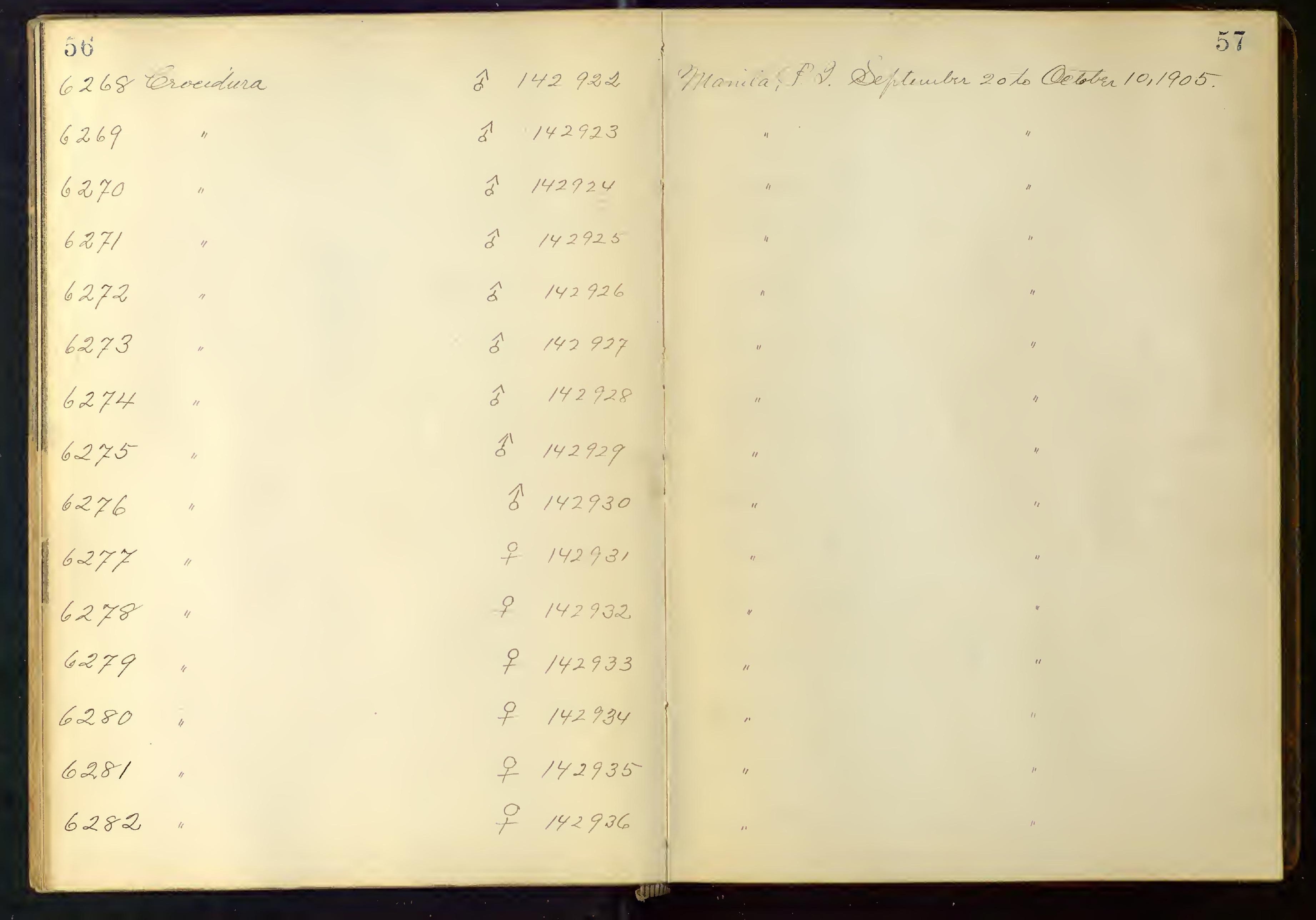




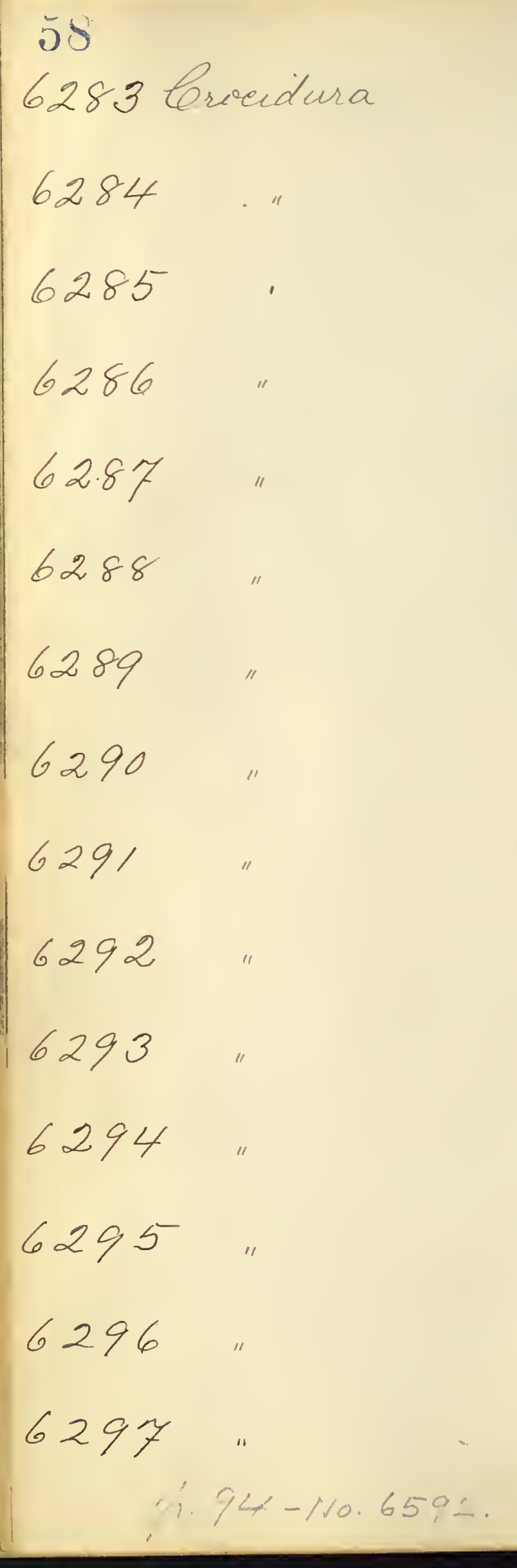

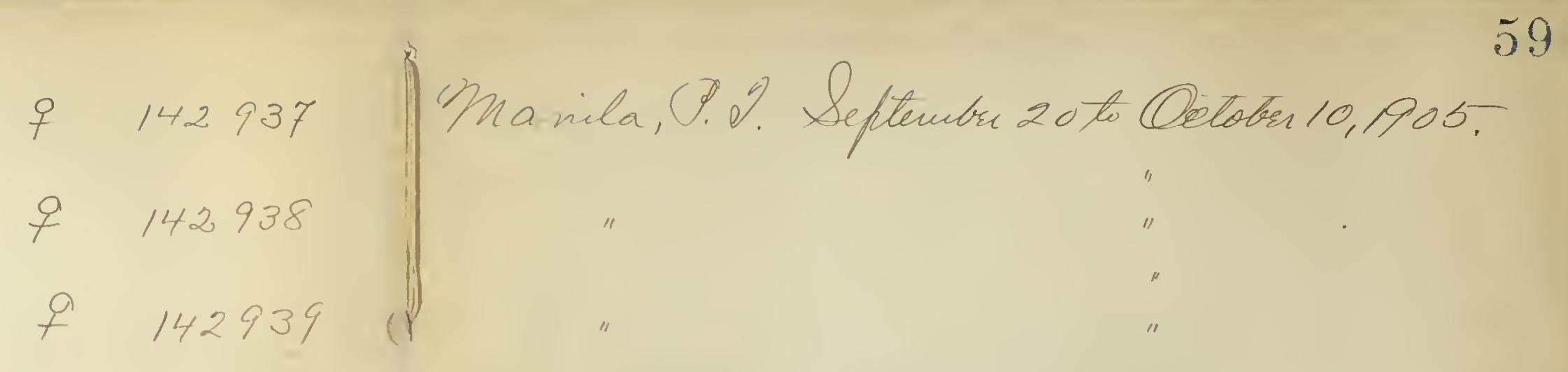

f 1172940

ㅇ 142941

f 142942

9. 142943

q 142944

$+142945$

‥120 946

f 142947

ㅇ. 142948

p 142949

$+142950$

9142951 
60 Mangan clearnig, left reide of Alag Rivir, Muidoro 6298.2 Mus

6299. Mne

6300. mue Eanif No.2. Alag Puir

6301

teynomolgu

mindane

\section{$x^{2}$}

Alagl Pwir. Winidow, at 450 fut. Yhor-81906. 6302 . Plus

$(144924)$ tad
$(144632)$ 9

6303.

6304

Oilangyan Lelearing, Sifte side of Alag Ruis Sf pur of main ridge, 3Ut. Halcon. Nov.14.1906

6305.

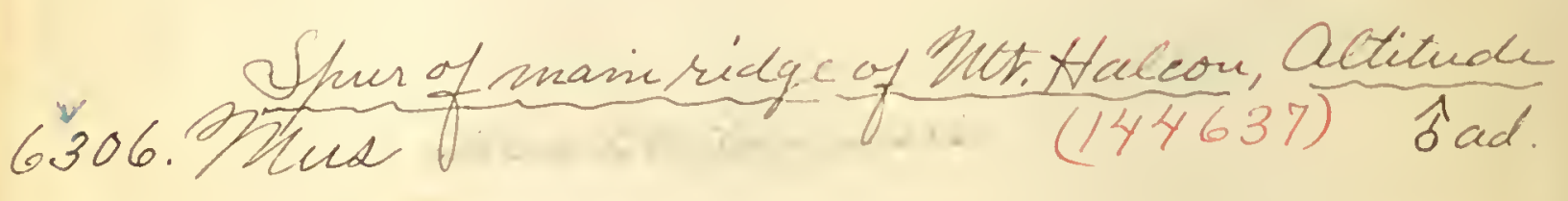

6307 . 6 orex.

6308.25 orex 6389 . DPMus
(144649) $\hat{\delta}$ ad.

$144650)$

tad.
sad.
Leland, P. . . Altitude 2250 feet.

Put in alcohol by Antonio. Mutal tag Yro.83.

, 1906.61

$$
\text { do Netal tag tho } 72 \text {. }
$$

do Unetal tag 2ro.81.

P.e. Altitude feit. Novmen 1906.

Stin and stulle. 1160;580;140;155;35;39; from nose to angle of month, 38; to eye, 47; to ear, 115t; gir the of cheet, 390. Anis yellowish brown. Bare space? whore sye, grajish white. Bare skrin of nuzzle light brown. Hect alive-flumbeous; claws phumbens-blaric Serotum browrist Slesh eolor. Wr......

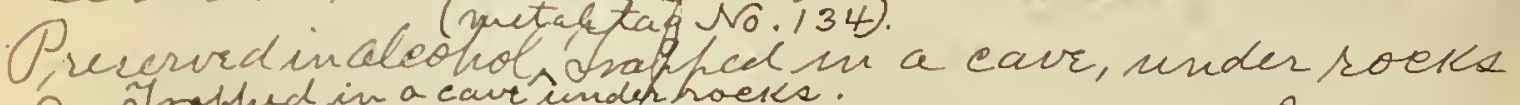

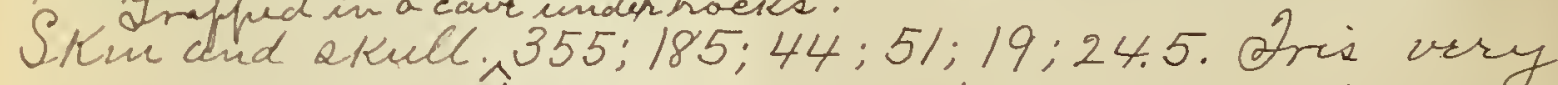
cask brown; fect flumbrous-furfile. Metaltag No. 906. Attitude 2250 flet. Novruber 1t,1906.

260:133;33:40;20;22. Anis viry dase brown. Thetal tay attached to skull, No.227. Irapped under a log Shot, where chinging to a banch where it was seen to alight, in the canf, at twilight. fect. Novenuber 15,1906.

$307: 166: 169: 32: 42: 13.5 ; 18.5$. Oail furflish brown, concolor. Naked areas flechy brourn, the fore feet halest and the cars daknest. Outer postrior tubrele of hind foot divided, conposed of two district tebercles, suaking 7 . For fect 6-tubreuldte.

$135 ; 68 ; 62 ; 15.3 ; 27 ; 5 ; 9$. haked areas dark furfish brown. For and hind fect 6-tubreculate.

Strin and stuell (bratcent).

Btini. and atuel. 
0.I Dhur of main ridge of 7ttr. Haleon. Altixude

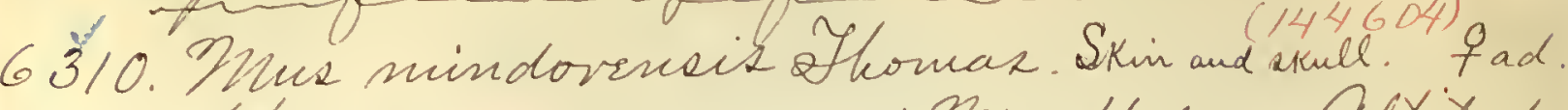
$\checkmark 6$ Hom of main ridge of Met. Haleon. Altitude 6312 . Porex 6 6313. Dorkx $63 \%$. Plue mindorensis 8thoncas (144606) $\hat{8}$ ad.

Efrur of main ridge of Nut. Haleon. QCtitude

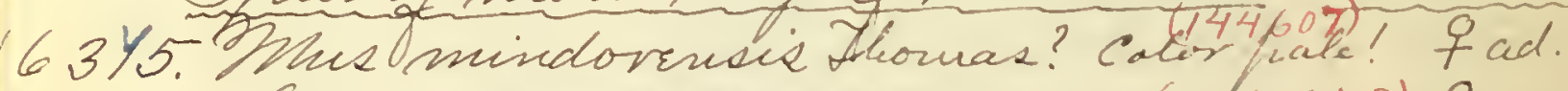
6316 Shrew. (144652) 웅 6317 . Shrew (144653) 9 ad.....

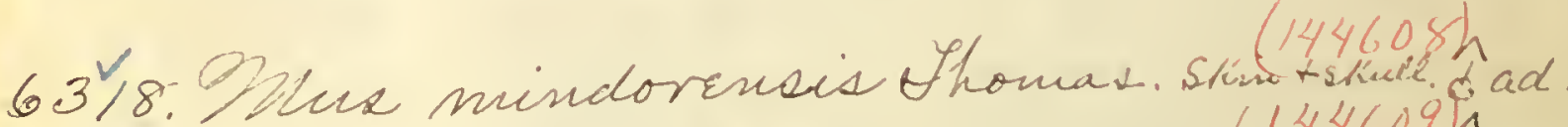
$63 \%$ " " " "44619) त्रेad. $63 " 20$. Hllux mindoreneis thomar. (144610) qail.

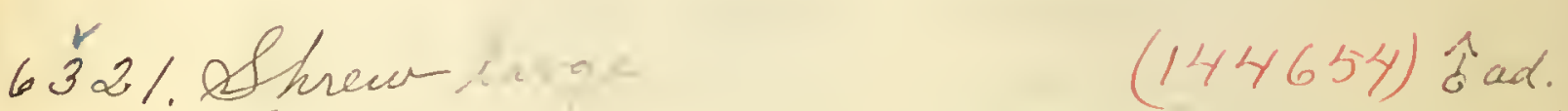
Shum of main indge of Prts thalcon. Altitude 6322 . Toynomolgue $\quad(144675)$ tad.

632.3. Porex a (144855) - ad. Oant zo. Trailersonig, sumuit of main
4500 dect. Novrubrr 15,1906

$310 ; 155 ; 158 ; 35 ; 45 ; 15.5 ; 19.5$. Ma $\frac{2}{2}-\frac{1}{1}-\frac{2}{2}=5$ fairs 4500 Leet. Noverubrr 16,1906

$205 ; 100 ; 28$. . . . -

An atcotol trotag. $130 ; 52 ; 53.5 ; 15 ; 26: 4 ; 8.5$. Laercal glande, developed.

4500 feet. Noveruber 17.1906

In aldohol. inotag. i... 1:.79;. 20

$361 ; 185 ; 188 ; 38 ; 50 ; 15 ; 22.5$. Ans vry dark brown Lipe ears, and feet frusfich brown. Srim and skell. 6300 tect. Novruber 18,1906. "Posifilate."

$317: 165 ; 34: 45 ; 13 ; 20$.

$128 ; 54 ; 14.5 ; 25 ; 4 ; 8.5$. Secth white

167:78:80;17:31; 4; 8.5. War nussing young. Wam $=$ mât 3 fairs, all inguinal. Feeth white.

$310 ; 148 ; 151 ; 33 ; 45 ; 13 ; 20.5$. Molting. Covlors licitu. 6320. $340: 176 ; 179 ; 37: 46 ; 14 ; 21$. 6300 fcet. Povrnerr 19,1906. "Poney Hlats."

$318 ; 158 ; 162 ; 33 ; 43.5 ; 14 ; 21$. Molting. Wose, lips, ears,

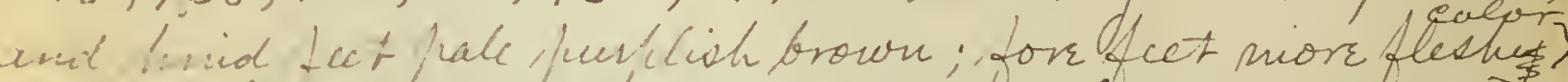

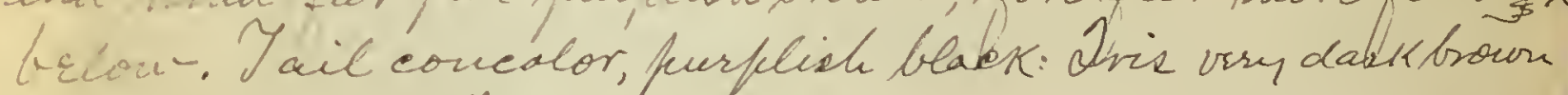
Zlot inegnant. Atkin and skull.

$163: 72: 76: 18.5 ; 30 ; 4.5 ; 9$. QLette white Body (No.121) . 6300 fect. Novenuber 21,1906." Posey Hats." $815 ; 1410 ; 420 ; 112 ; 106: 30 ; 35$. Matud parts-nose, face, ears, fect - purflish brown, exceft palfebral requor whlè is bluish white ax is a mall shot on anterior base of ear. Mannuas 2 pair, pectoral

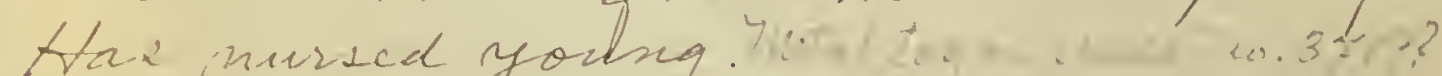

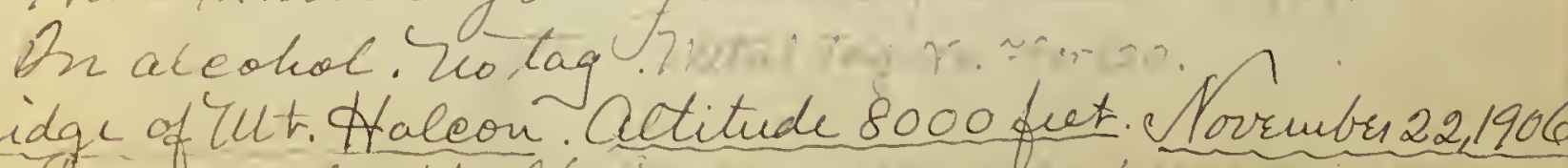


61 Sfhur of main ridge of Utr. A aleon. Altitude 6325 . Ontur mindorisisic Shoular. 611 ôd. Hhur of main ridge of Mot. Halcon. Altitude

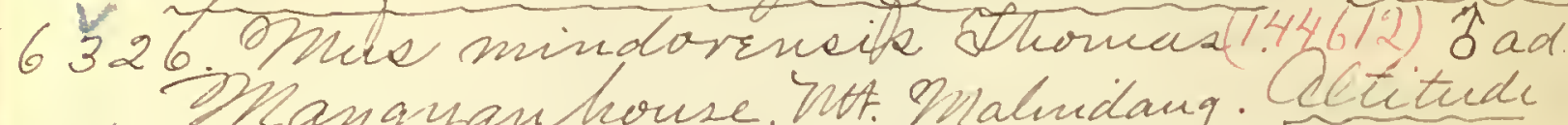
$6327.9 m i s$

$6328.27 m x$ 6029 . $(144623)^{3}$ tad.

6330 . Alag Puir,

6031 . Mus

6332.2 mie

6.33 .9 ine 6300 feet Novruber 233,1906. Soser Hetata."

6300 fect. Povenubr 25:1906. "Pory Flats."

3000 fuct. Noverubr27,1906.

$385 ; 208 ; 210 ; 38 ; 53 ; 18 ; 23$. Shin and skull. Contained 3 large fortuses. Frapfed manarpield Aris dark brown. Aket purplish Jlesh colotic Cawz White. Ears flesh color at base, purplieh brown a fieally. Bare skin around nose and mouth halc Jur kiak brown. Jail unicolor, darkfurflish

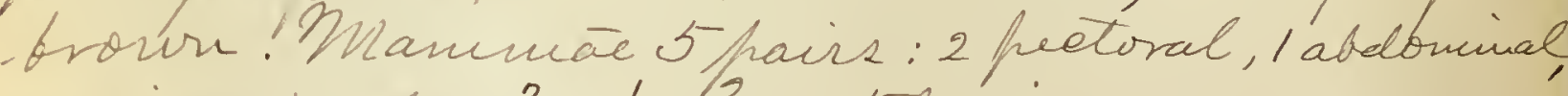
2 ing ninal $-\frac{2}{2}-\frac{1}{1}-\frac{2}{2}=5$ flaik.

ail" qone; skult booken. Anid foot, 38 mun. Sk. t skele Alag Puir, Mindono 2d, P.9. Cttitude 2250 fect. Nov.22. $390 ; 205 ; 212: 38.5: 51 ; 15 ; 21$. This Pat differe from thore collected elsewhere. orie vary darterown Fist and claur flesh color, Eare, nose, and lifis hight furfilish ofown. Urider frasts white fanitty washed firth yellow. Skin and skull (broferen). fut. A Novruber 30,1906. Camp no

340;183:185;39:47(bromincead); 17;23.5. Belly light brown. Stin and skell (broken).

mature? Tettes amall). 358;186;189; 39.5;44;19;22 Relly yellowish white. Skin and arull. $385 ; 201 ; 206 ; 39.5 ; 51 ; 16 ; 22.5$. Belly ercamy white. Lifra and fect pale furplish flesh color; Claws white. Hose and care pusplish brown. 2.68;142; 33. Belly ercamy white. Sarre specie as abovr? Bare farts colored the same. Bkitt srule 


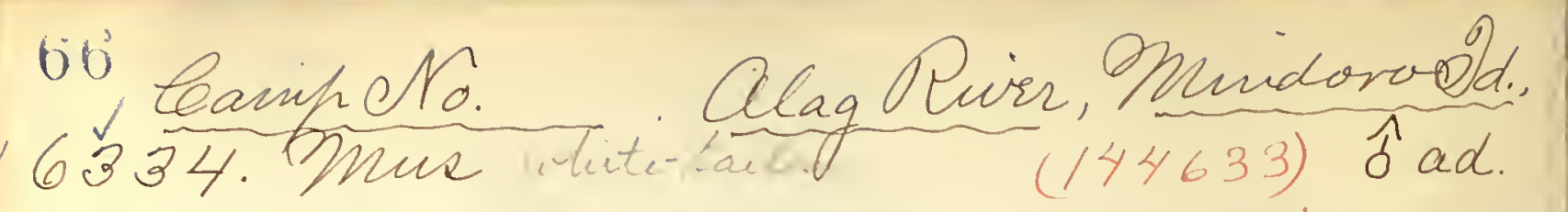

$6335.974 x$

$(144630)$ iad.

6336.7mus

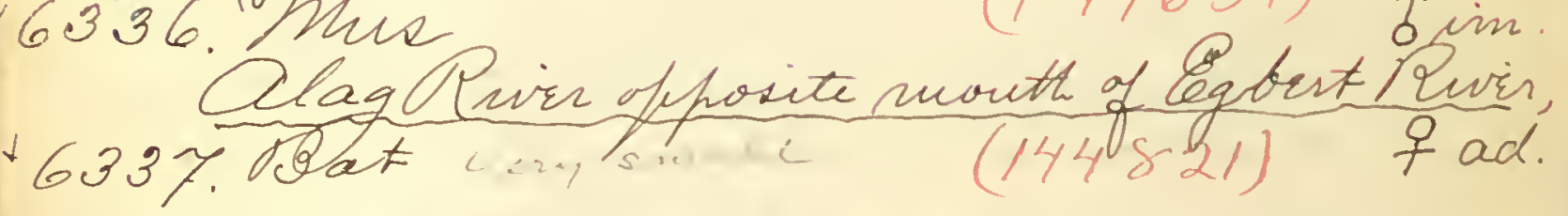

60338.9742

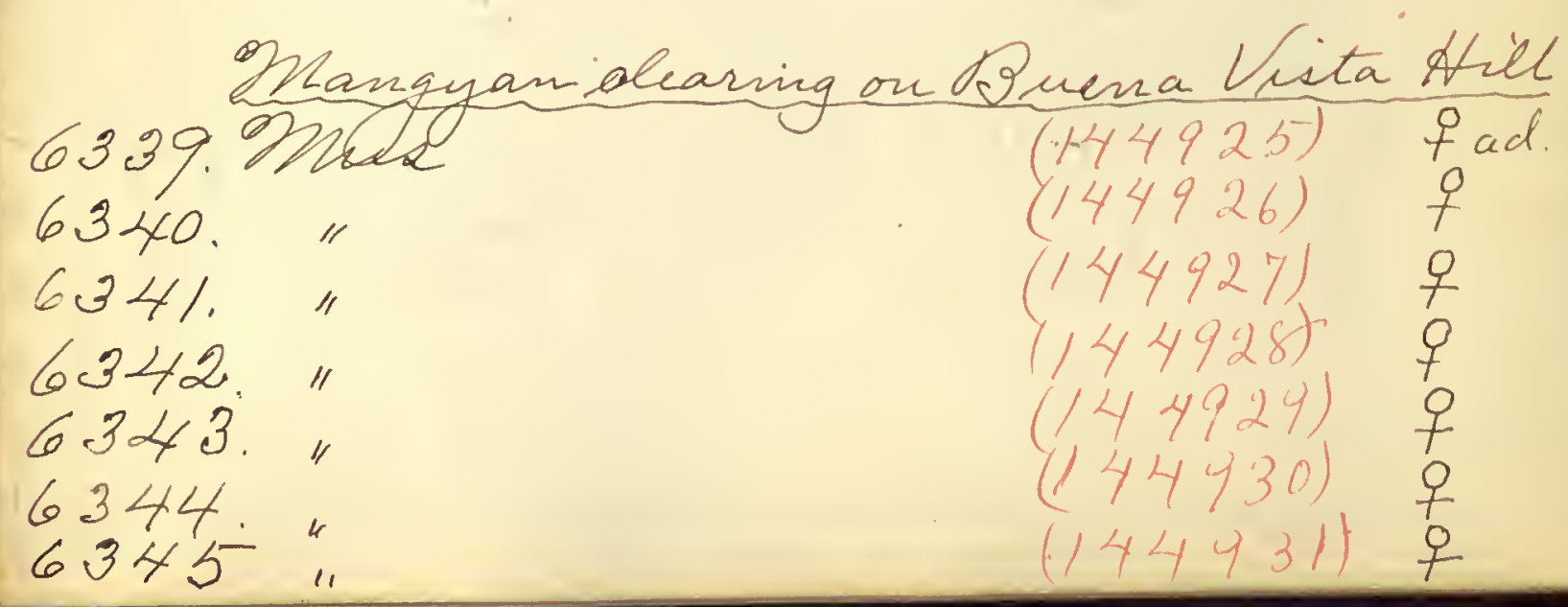

Q. Q. Altitude feet. Novmubr $30,1906.67$ 450:241 (tif naride); 48:61:21:26; white hortion of tait, 125.2 ris dark brown. Fect dak purplesin brown; claws furplish flesh color. Bars, eyplide, nose, and lifs paler futhphich brown than feet.

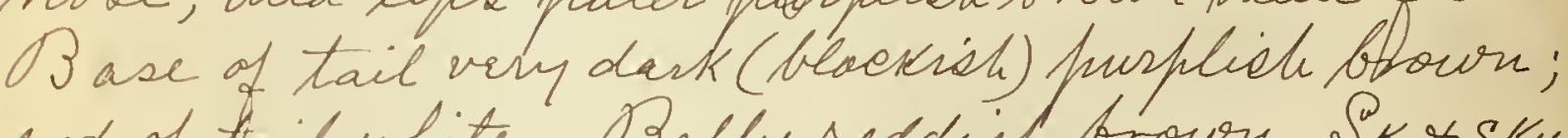
ind of tail white. Belly reddich torown. Sr.Y shewe. Feet, lifs, and clatus fleah dolor. Hose, eyelids, and cars purfelish bfown. Oris dark brown. Mlammat 5 fairs: fuetoral 2, abdonnial 1, inquinal $2\left(\frac{2}{2}-\frac{1}{1}-\frac{2}{2}=5\right.$ pains $)$. Containied 5.fortuser (3-2). Miasurements: 436:227:231: 390, 54:18.5;23.5. Sim and sruel.

330:175:38. Alitude fut Deceubr 2,1906. Dength, 40; al ar expanse, 244 ; fingers: Ist (thumbt),

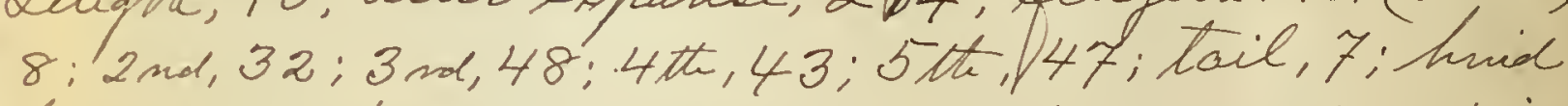
hint, 33 ; head, 16; car erown, 13; nated stene of head, flesh eolor.

$280 ; 149 ; 151 ; 29 ; 36 ; 13 ; 18$. Belly white. Fore flet and claur seshe color. Arind flet clothed

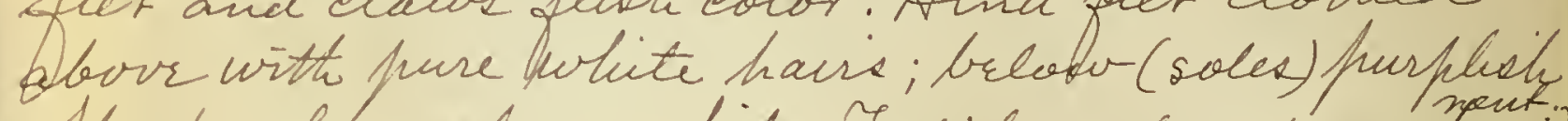

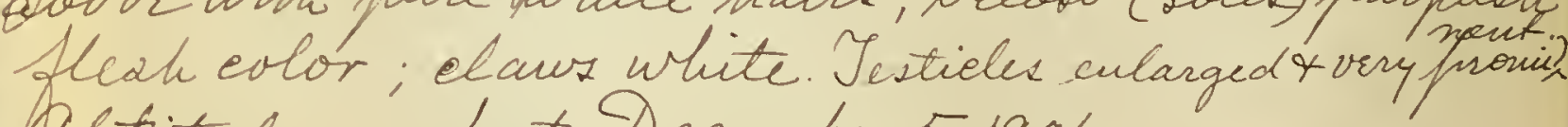
Detitude fect. Deenber 5,1906. Preserved ist alcohol. Wletal tag vo. 4084. do do dor
76.

74.

d's

73

77
78 
¿s Calahan, Windort daland, P. Q)

$63 \%$. OPlux

(144576) $\hat{b}$ ad.

6348.

6349

6350

Ceauglt aboard the UsA.Y" Inindanao", $\checkmark 635 \%$

635\%. Baquiv (leanff John Aay). Benguet Provine
6352 . Saradoxurus.

Batae, Llocos horte Provine, Luzon 6352 . Bteropus (144761) Gad. 6353.

6355

6356

6357

6358

6359

6360 .

$(144764)$ tad.
Altitude zofect. Decenber 8,1906.

69

$475 ; 215 ; 280 ; 46 ; 59 ; 16 ; 21.5$. Manumae 6 prairs: 3 thoracie, 1 abdonimal, 2 ingrinal $\left(\frac{3}{3}-\frac{1}{1}-\frac{2}{2}=6\right.$ pairs). Ceanght in a dureling

$470 ; 230: 234 ; 46 ; 57 ; 16 ; 21.5$. in the Chinia Hea. Decuubur 22,1906.

Total length, 170; tail, 90.

- Luzon 2iland, P. ?. Actitude 5000 feet.

Sim, reull iuside. Shot retunied fy leontiact Sirgeon Durray, Us. Anny

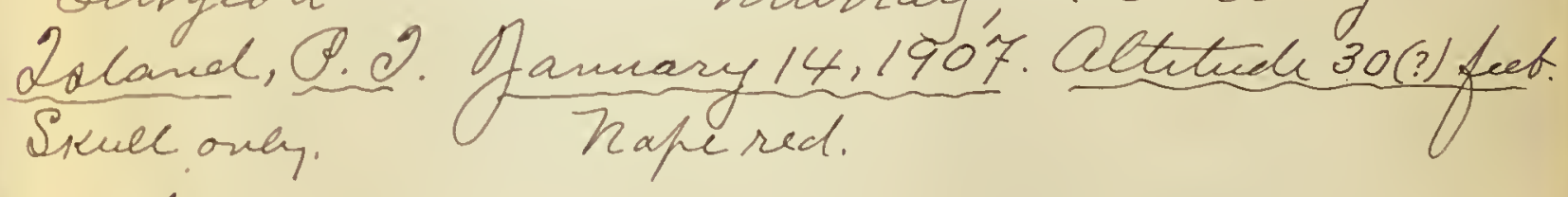

do

(144765) tad.

(144766) qad.

(144\%67) 余ad.

(144768) foad.

(144769) 今ad.

Strie

do arull. "red

do

do 


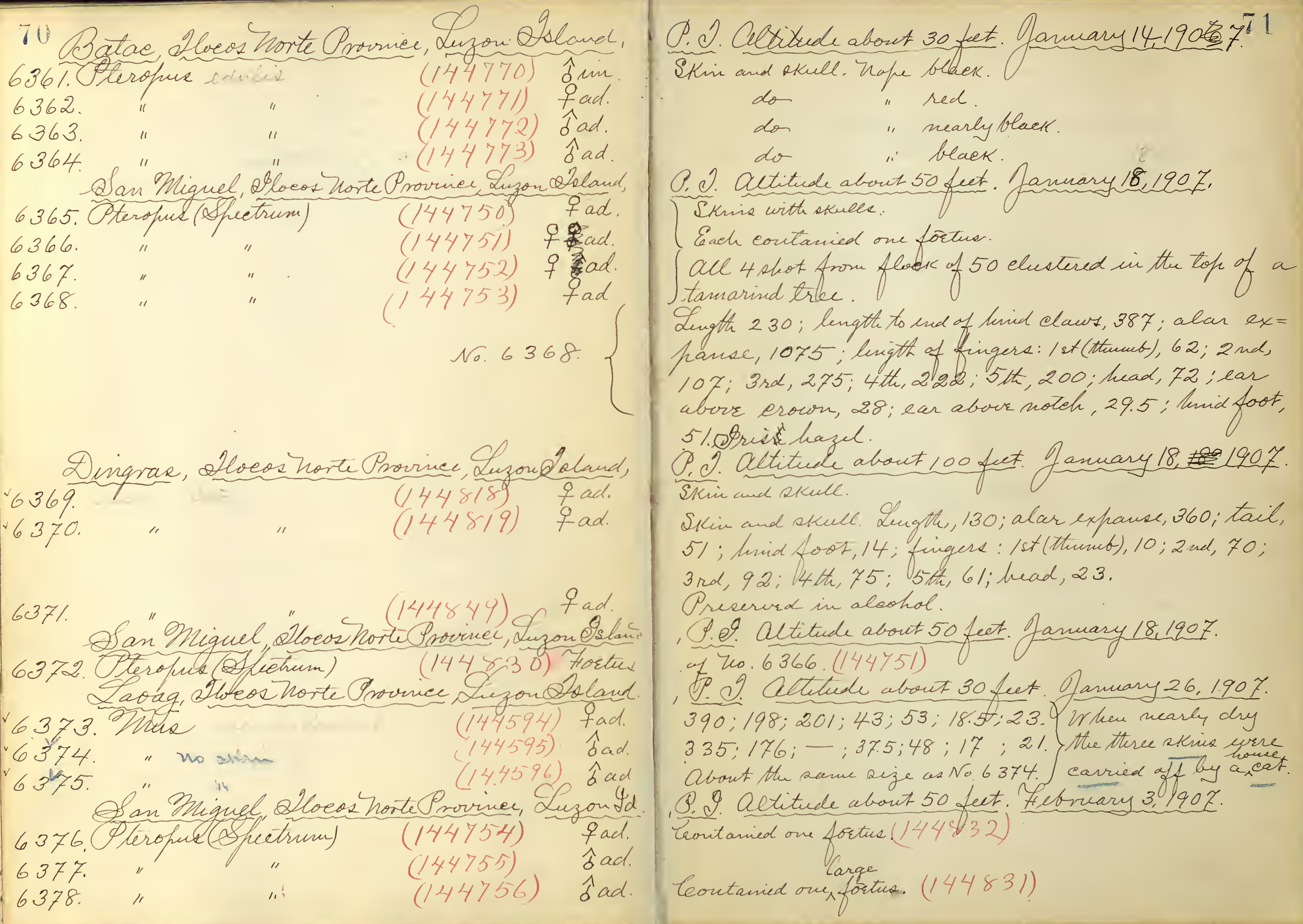




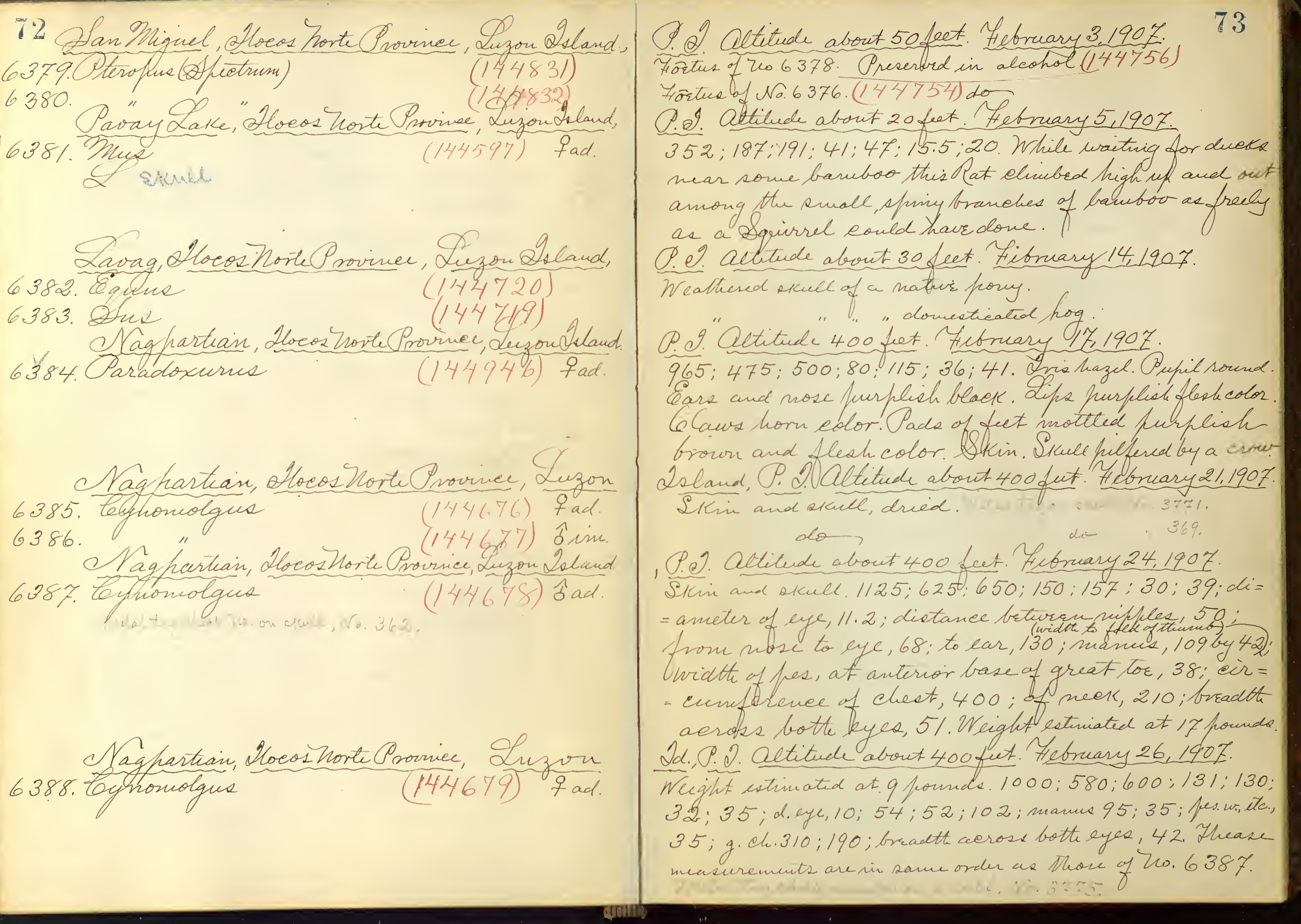




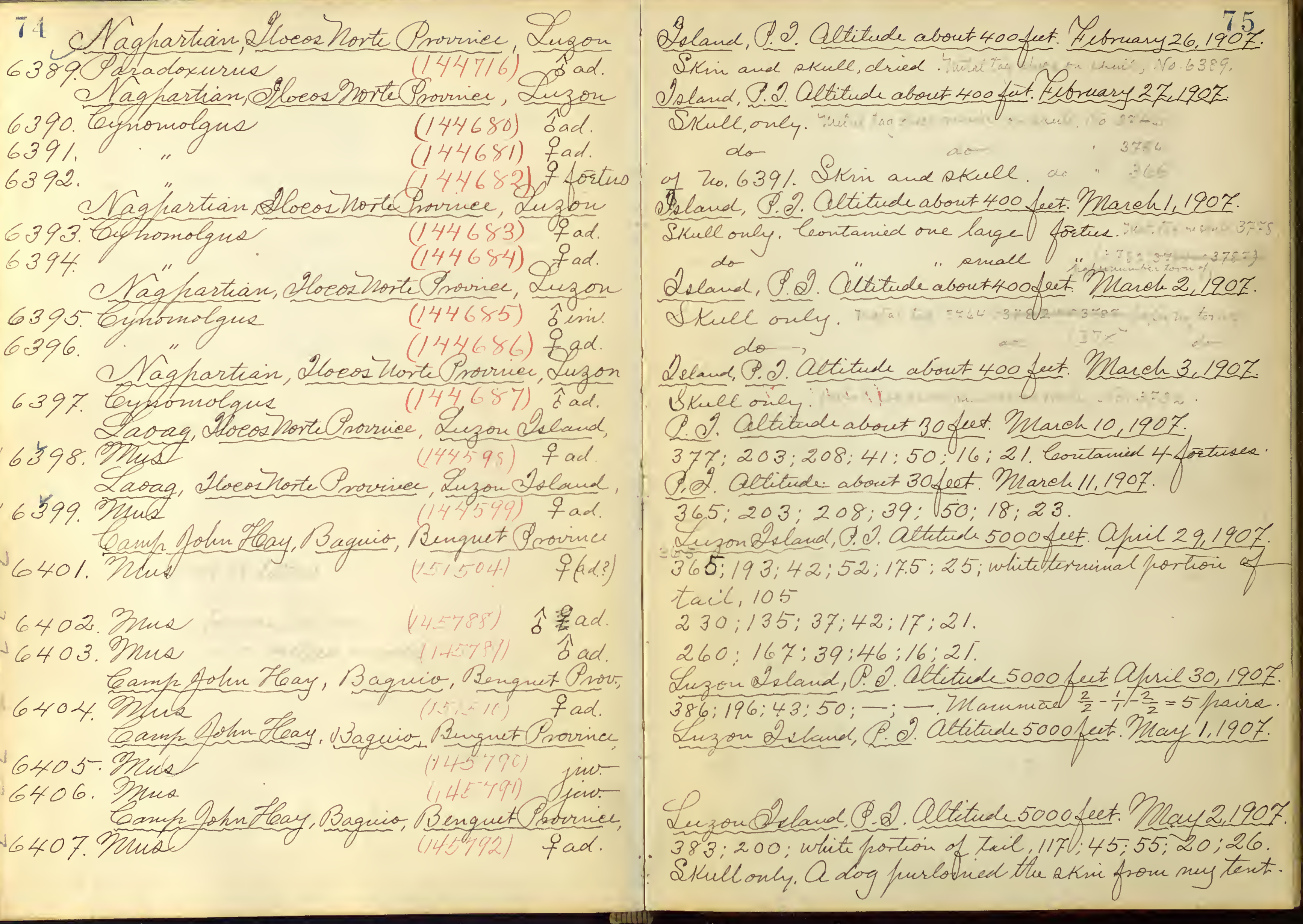




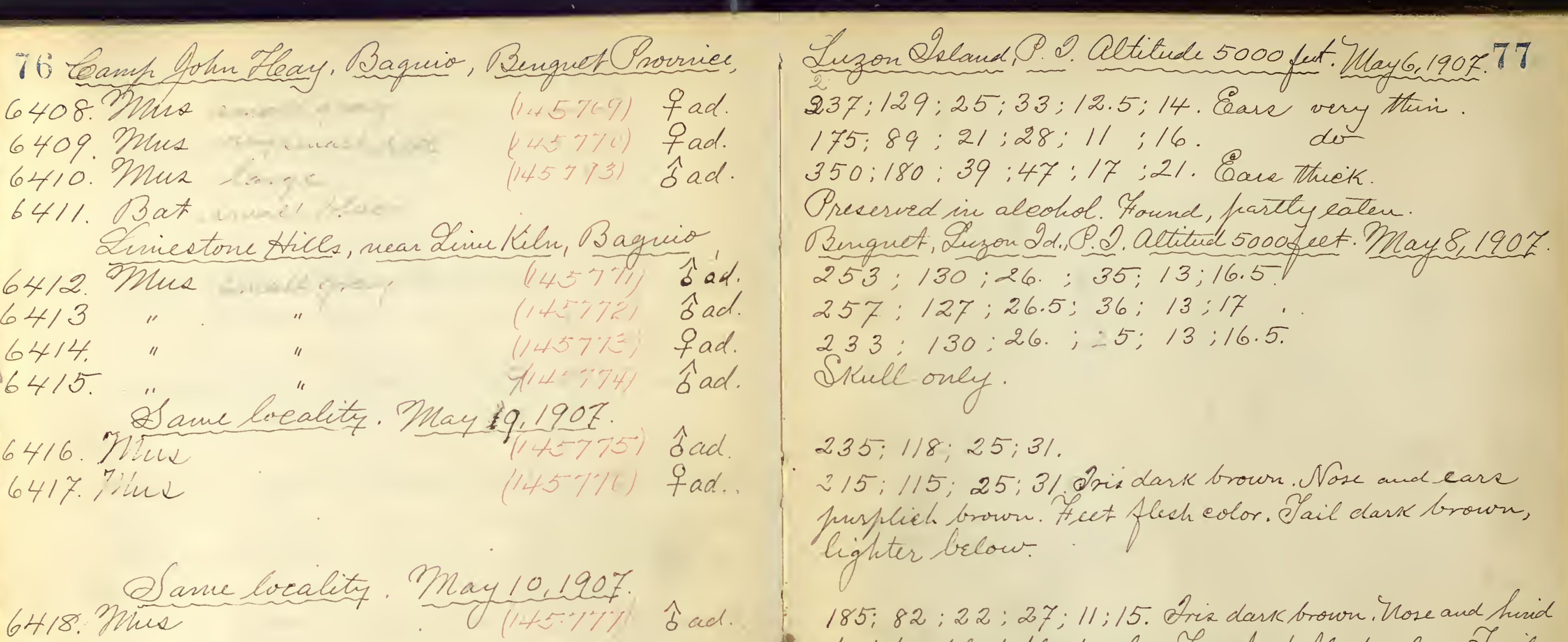

6418. Muse locality. May 10,1907.

185; 82;22:27;11;15. Ariz dass brown. hose and hind fect prurfich flech color. Fore fect flesh eolor. Ácil furplide brotur above, flech dolor below, Ear furplich

6419. Pus

$(145778)$ ô ad. $255 ; 130 ; 27 ; 33.5 ; 14 ; 20$. 2ris dark brown. Ears kusplish brown. Fore fect fluch eolor. Arid fect purphish brlow. Tail dark brown, slightty pden brlow.

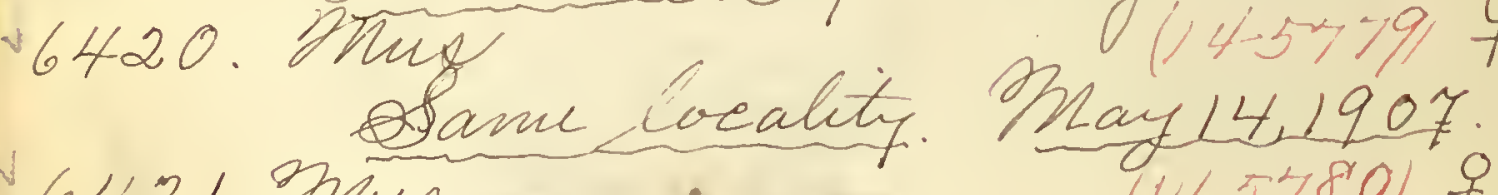

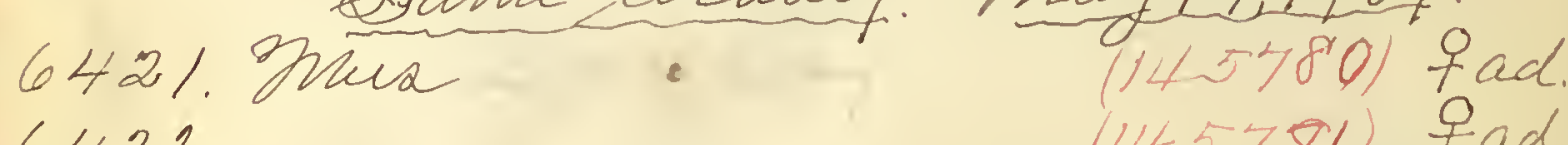
$243 ; 123 ; 25 ; 31 ; 13.5 ; 17$. 6422 .

Skin and stull.

06423

"Same locality. Mnay15,1907.

do

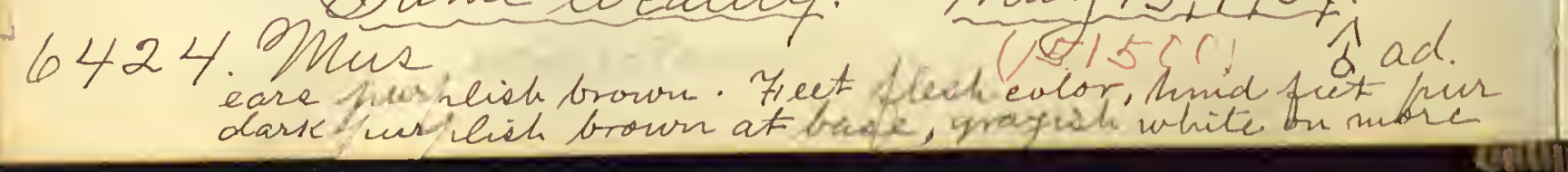


78 Limetore Hbill, mar Lime Kiln, Baguio,

6426. Dnus

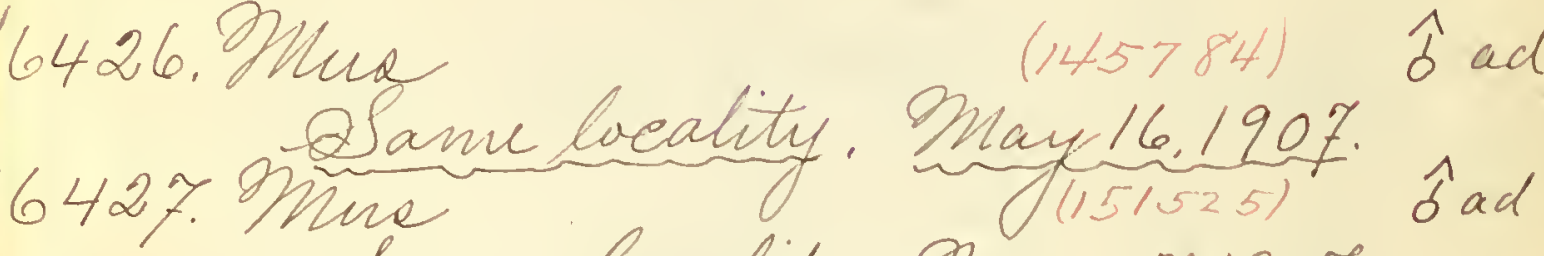
6428. Onus beality. Mnay 17.1907. 6429 6430 6431. 6432

6433. Mus "Same beality. Mlay 18,190\% $\mathrm{o}$ ad. 64344. Mme beality. Mnay 19.1907. qad.

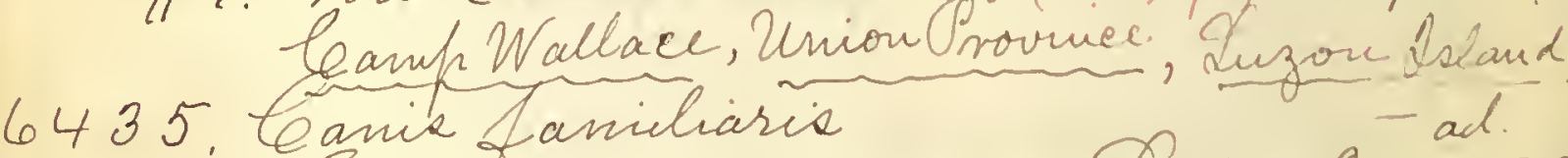

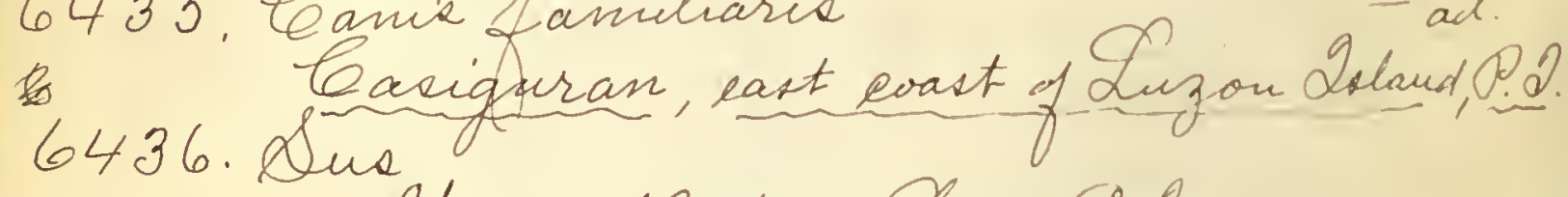

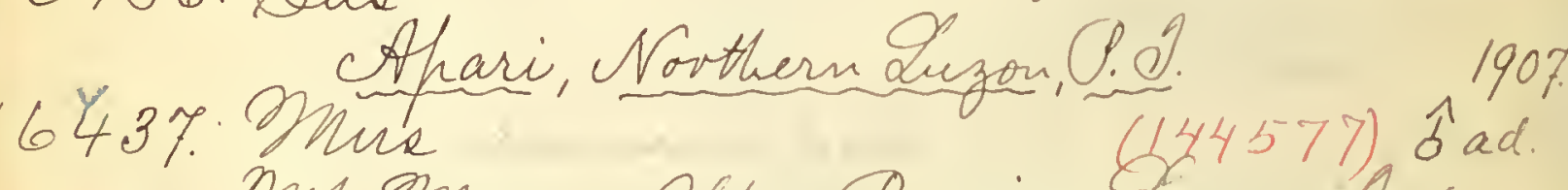

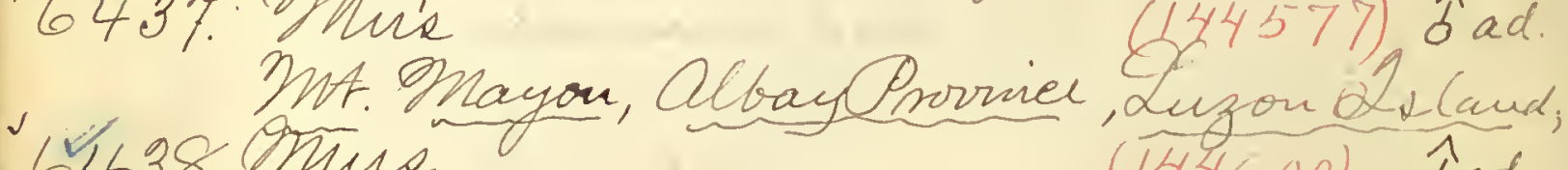
6438. Mure 144600$)$ îad.

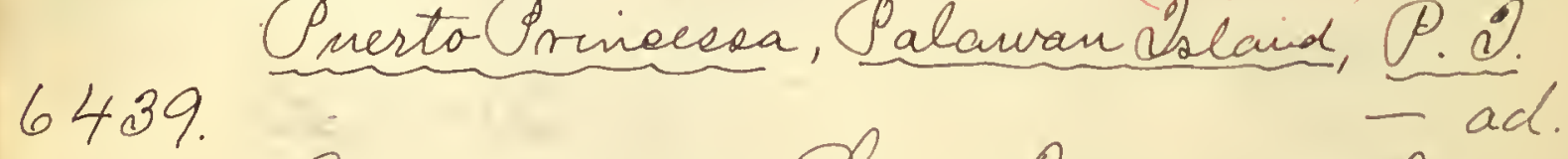
6440.

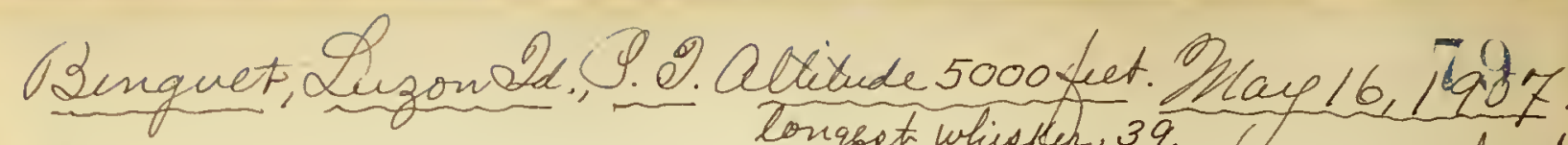
$239 ; 133 ; 25 ; 34 ; 11 ; 16.5 ;$; min dic dask brown. Feet flech color receprig soles of hind fut which are furfhisth. Evail dark brown, flaeer below, Bars purphish brown,

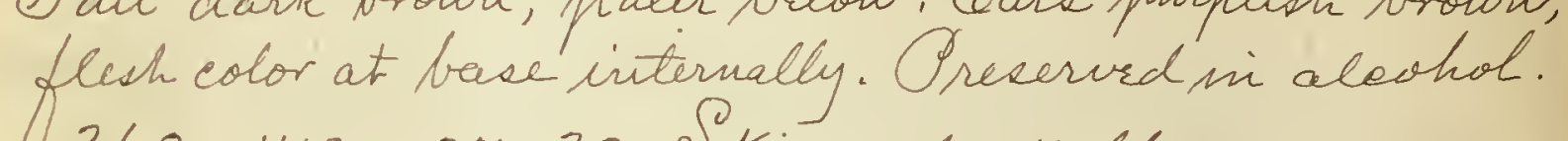
260;140;27:33. Skim and skull.

$245 ; 130 ; 25 ; 33 ; 13 ; 17$. Preserved in aleohol.

$270: 137 ; 27: 37.5 ; 13.5 ; 17.5$. Lange.

$252 ; 128 ; 26.5 ; 35 ; 13: 17$;

$232 ; 123 ; 24 ; 33 ; 13 ; 16.5$.

Rotten. Hrad in aleohol.

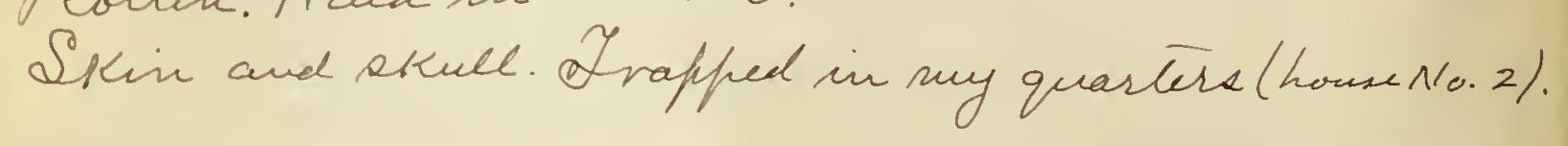

$435 ; 225 ; 229 ; 41 ; 51 ; 18 ; 23$. Skin and stuel. Thannuñe 5 pairs $\left(\frac{2}{2}-\frac{1}{1}-\frac{2}{2}=5\right.$ paira $)$.

Stin and (Prull in aloohol).

P.2. Allimde 30 fect May 25,1907.

Frapment of upper jaur

Actitide 10 fut.

Weatherced berull of pig; ; frobably wild.

Q. 2). Actitude 4000 fect. Jume 5,1907.

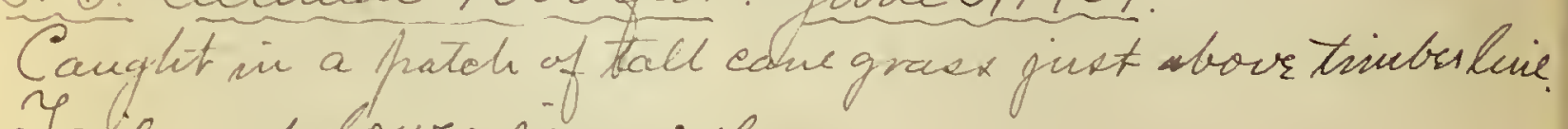
Sail and, Courn jeour, only.

Sume 7, 1907.

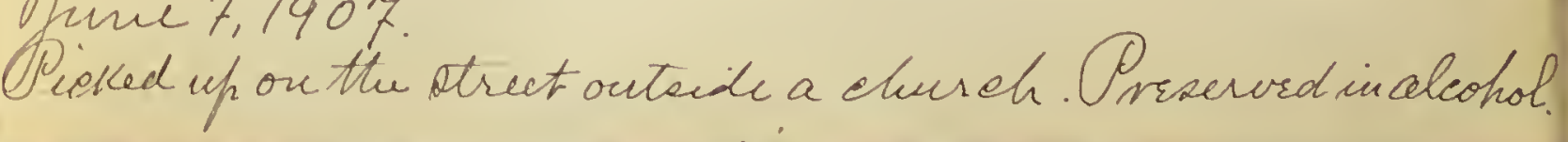


SU Malabou (nem Marila), Luzon 2oland, P.Q? 6441. Chrocidura.

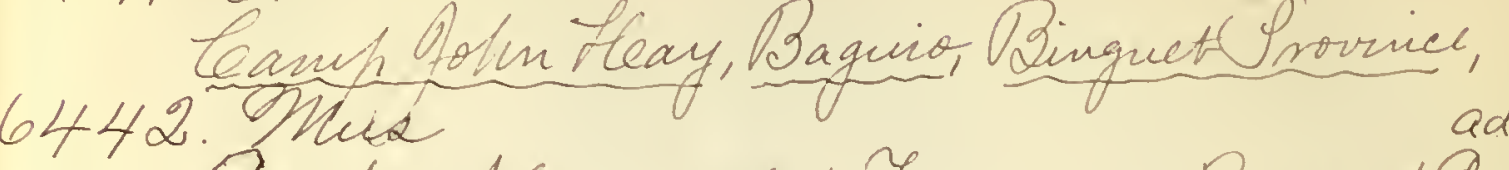

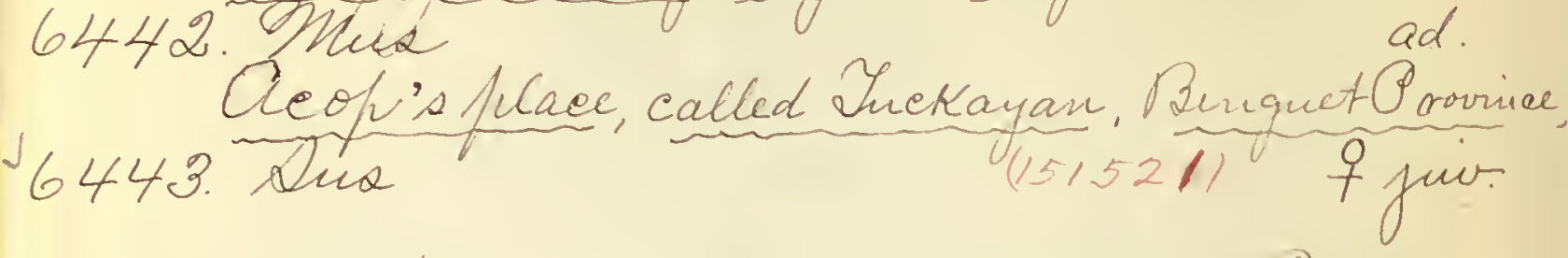

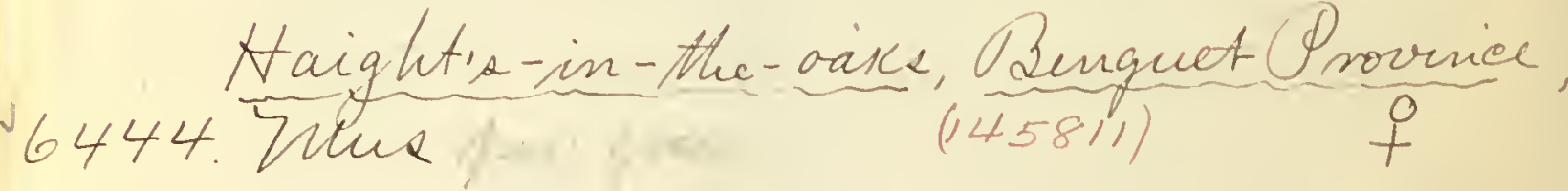
6445. Same locality and datc.

Same beality. July 23,190\%. 6446. Campr folm thay, Baquio, Burguet Provme 6447. Mtur. Note. - Ceriaco fut fool numbira on the numbris of tag attached to foot. 1644.7. Haightin-in-the-aake, Benguet Province, 6448. Phus 6449.

$\hat{a}$ ad.
Abtitude 10 feet. Opril, 1907 .

81

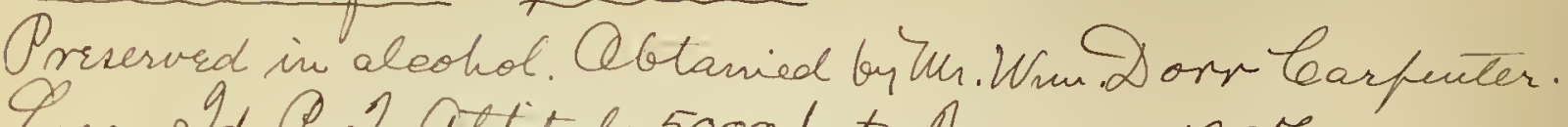

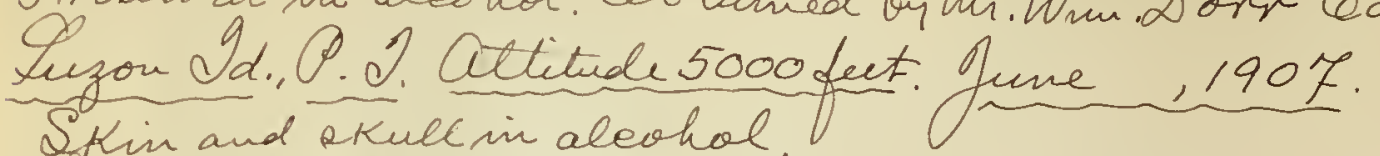

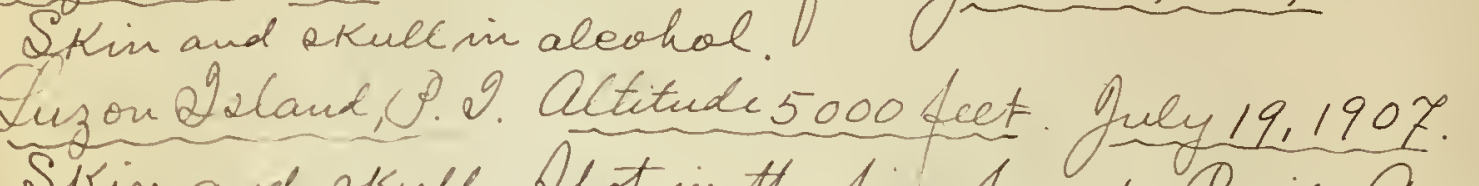

Sirin and okull. Bhot in the fine forrat. Paid Acop $\$ 6$ gold for it. Mistook it for a wild Pig. Aizon Qslaus, P. . . Altitude Yobofet. Guly 22, 1907. 260;135;138; 24.5; 37:14; 18. 2nir dask hage, Ban hale, purftish flesh color; claure horn color. Bane skin of tail, bstursen hairs, purplist brown. 3 ant nae $\frac{2}{2}-\frac{0}{0}-\frac{2}{2}=4$ pairs.

$270 ; 133 ; 136 ; 28 ; 39 ; 14 ; 18$. Ani dank hazel Eare furplish brown. Stin of tail, betursu hair, fun= - Huih brown. Feet and claws flesh calor, aolee purplich brown.

A. .9. Altitude 5000 fut. Grly, $190 \%$.

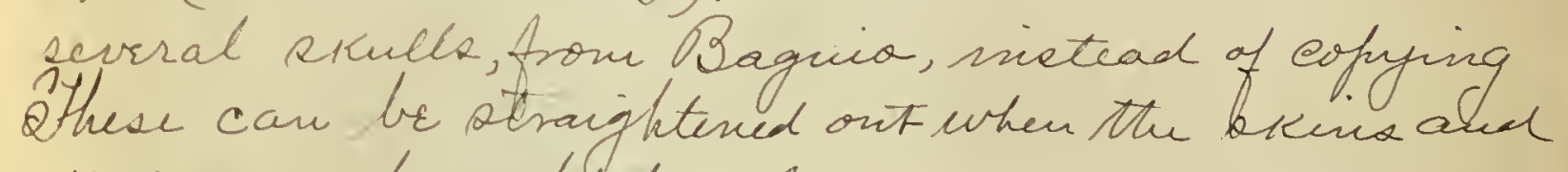
zKulls are brought to gether.

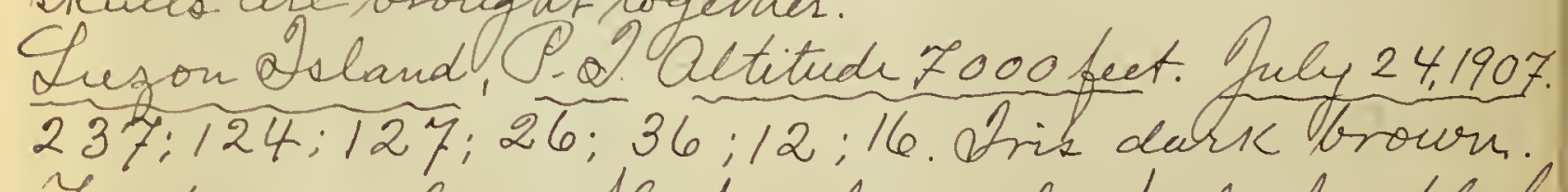
Fect and claws flech color soles pale furphes. Eare, nose, and ttail furplieh boown. Mammase 


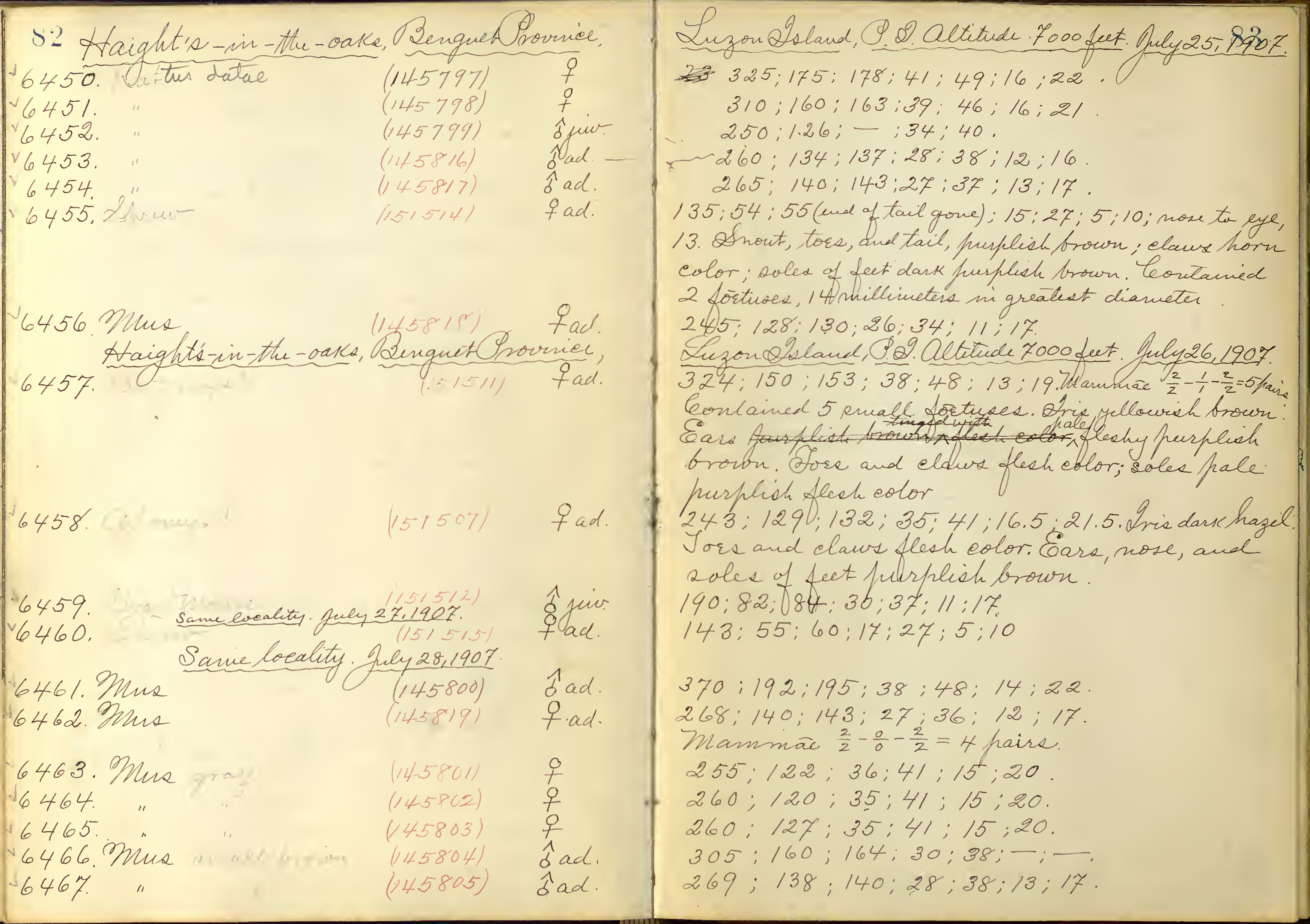


81 Haightis-in-the-oake, Benguet Provine, 6468. Ome 16469 . Dma 6470. mue $647 \%$

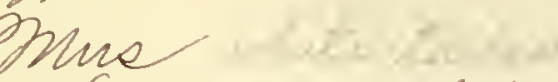
Same beality. Auly 2q,190Z. $6472.27 m s$ 6473 . Onue 6474 . Durs 647 紫5.

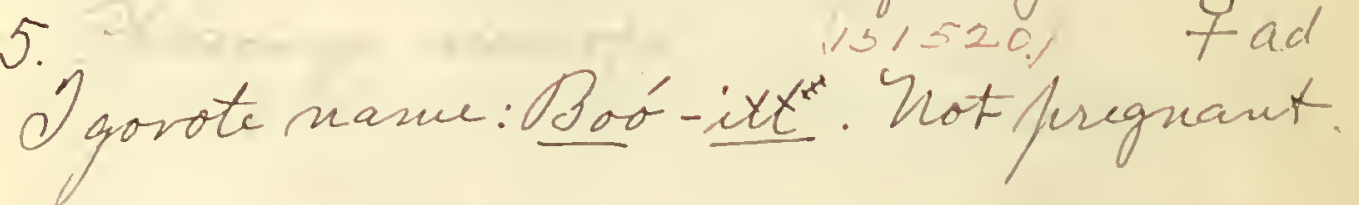

Luzon QDland, 9.9. OotitudeFooofut. Puly28,1907. 85

$270 ; 140 ; 144 ; 27 ; 36 ; 12 ; 18$. Mammae $\frac{2}{2}-\frac{0}{0}-\frac{2}{2}=4$ frairs $265 ; 131 ;-; 27.5 ; 37 ; 13 ; 18.5$.

$450 ; 240$; white portioin of tail, 113;245; 49;58;20;28.

$-212 ; 108 ; 110 ; 26 ; 32 ; 10 ; 15$. Shie rfeciés was

ouly tound in banks of a nueadow

$193 ;(103 ; 105 ; 24 ; 29 ; 12 ; 16$.

$\{265 ; 133 ; 136 ; 26 ; 36 ; 12 ; 17$.

Inamae $\frac{2}{2}-\frac{0}{0}-\frac{2}{2}=4$ paire.

$820 ; 400 ; 428 ; 98$; width of fer, 33; 303;21;34;

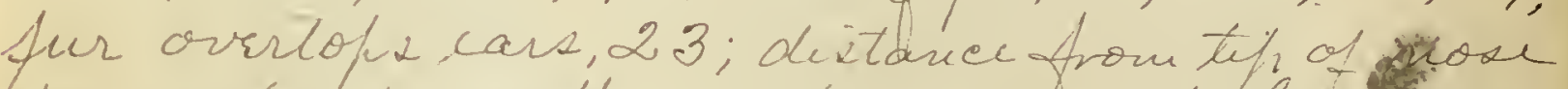
tto anglc of moith, 43; to eyc, 47; to base ear, 84; diameter of sye, 9; longest whisker, 106. Wcight, 4 pounbls avoirdufiois. Tthe young cling to the two nigunal teats, and are dragged by the mother. Mr. Haight sow one (1) young bing dragged in this way, which causis a imareed Mongation of the teats of millifara. IV heu not sdekling foung the thats colafac. withric the mamnary of land, rut may be

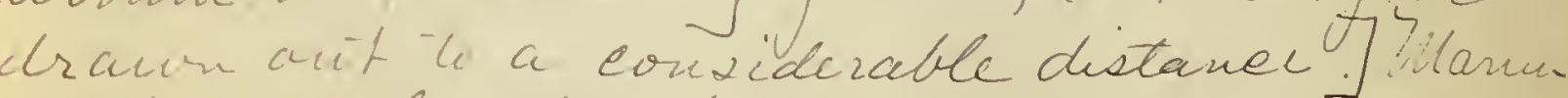
63.1.y 26 ; length of hind claws (chord): 1st, 11; 2nd, $15.5 ; 3 d, 15.5 ; 4 \mathrm{NA}, 15.5 ; 5 \mathrm{Nh}, 13$. Aind elaur: Ist, 3.5 (a Alattened mail); 2 nd, Groken; 3nd, 10.5; 4 the, $11.5 ; 5$; th, 10. Length of uffer ricisore from qume, 9; width of hair at gune, 10.5; becer

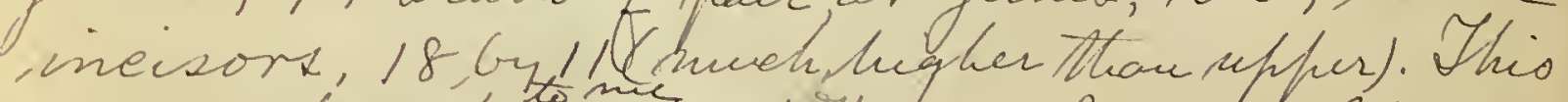
one was tranght trive, fione an 2 gonte. It ate Leveral bodeles of akiunded birds at might (curning) and the larger part of a eamole ni the mopningo. 2talzo drank water frcely. Durng the night it of. 


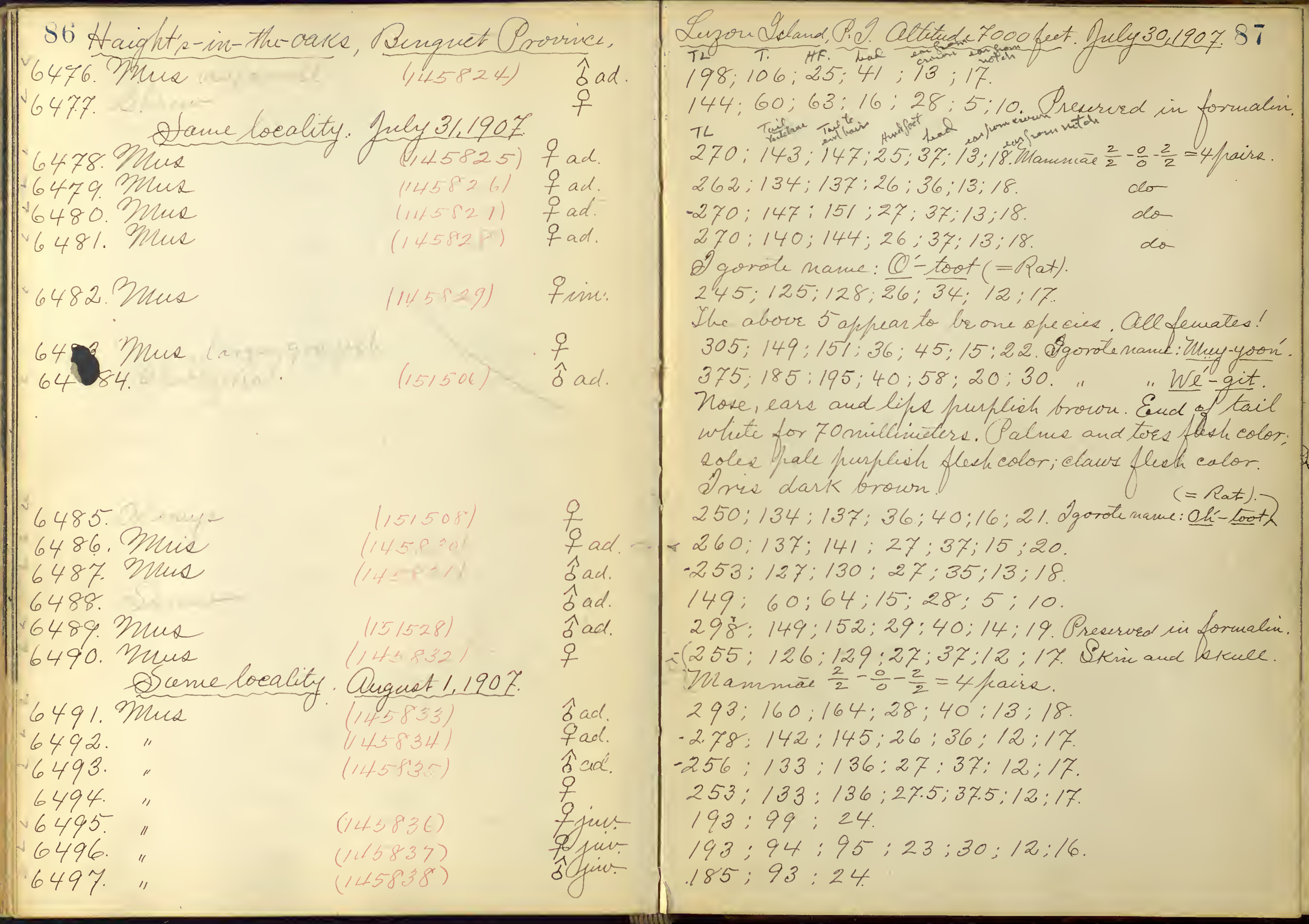


88 Naightin-in-the-cakls, Benguet Provind 6498.atroney

16499. Omus utitithend (151502) qad. $\checkmark 6500$. Pnux 6501. Mmas 6502

6503

6504

6505

6506

6507 . Mun

$6508.07 m$ m

6509 . Bma $651 \%$

6511 . 2 ins

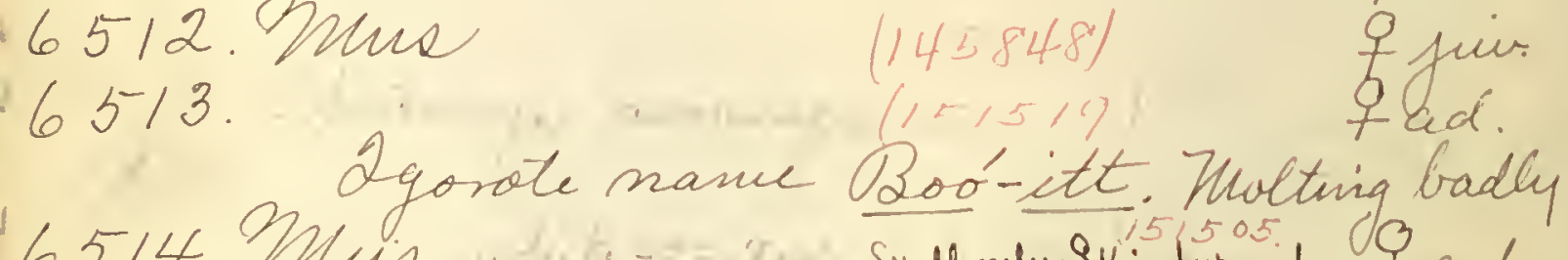

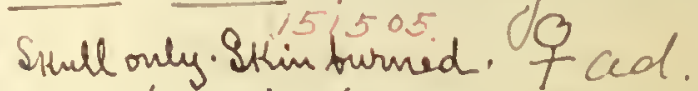

6514. Mnv

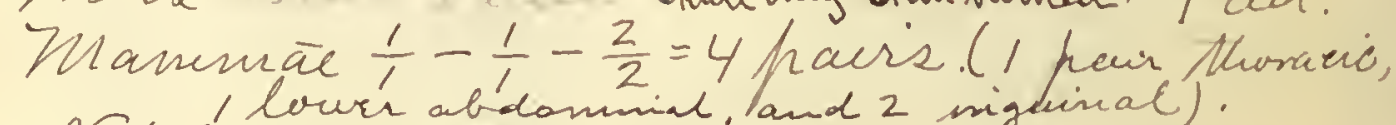

6515.3 mine

6516

$651 \%$
6518

6519.97mes
Luzonodand, Q. A Atitude Fooofect. Ang., 1907.9 $285 ; 150 ; 153 ; 38 ; 44 ; 18 ; 23.5 ;$; 1 mic brown Earx, nove, hind Roles, and tail furplish brown.

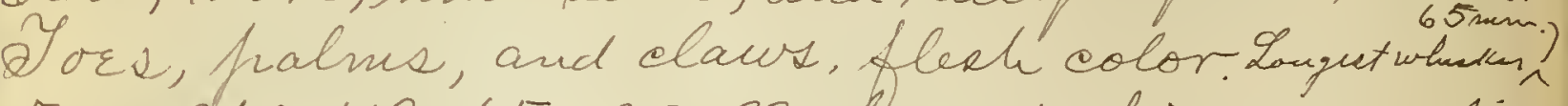
$500 ; 265 ; 49 ; 65 ; 23 ; 28$; bongest whisker, 80; white fortion of tail, 155. Mnammae. $\frac{2}{2}-\frac{0}{0}-\frac{2}{2}=4$ paire.

$402 ; 210 ; 47$

$-266 ; 146 ; 150 ; 28 ; 36 ; 12 ; 17$
$252 ; 133 ; 136 ; 27 ; 35 ; 12 ; 17$. Preacerved in formalni $255 ; 130 ; 133 ; 27 ; 36 ; 13 ; 18$.

$248 ; 131 ; 134 ; 26 ; 36 ; 12 ; 17$

$174: 78: 24$

$143 ; 68 ; 72 ; 16 ; 28.5 ; 5 ; 10$. Suitabe for a type.
$255 ; 129 ; 133 ; 26 ; 36 ; 12 ; 17$.

$265 ; 135 ; 138 ; 27.5 ; 37 ; 13 ; 18$.

$260 ; 142 ; 145 ; 28 ; 36 ; 12 ; 17$

$246 ; 135 ; 26.5$
$261 ; 126 ; 129 ; 27 ; 37 ; 13 ; 18$.

$182 ; 83 ; 30$. Fuct very ptout!

$560 ; 130$ (and gone); $92 ; 106 ; 30 ; 36$. Waight ty fornde

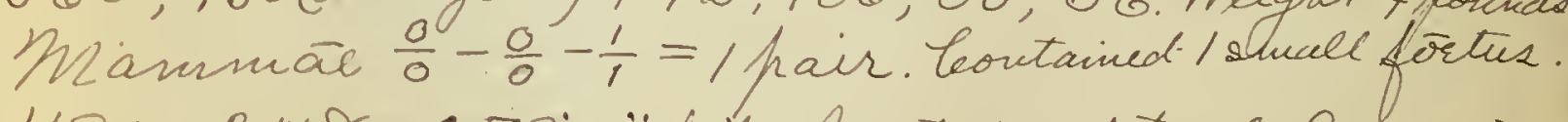

$480: 2.48,+252$, w white protion of tail, 95,55 ;

$70 ; 26$; 34; nove to eye, 34; to car, 60; to ti, of

ear, 94 ; diancter of eye, 8.6. Ogarote mame: OL' Toot.

creverd ni formalm.

$280 ; 142 ; 145 ; 29 ; 39 ; 13 ; 18.1$

$262 ; 134 ; 137 ; 27 ; 38 ; 13 ; 18$.

$300 ; 167: 170 ; 29 ; 41 ; 14 ; 19 ;$ mone to eye, 17: to car, 33; to tif of ear, 53 ; diameter of efe, 5 . 
YU Haightis-in-the-oaks, Benguet Proviner,

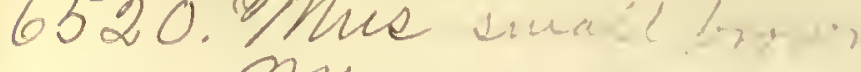
6521 . NMU⿰

6522

6523

6524

6525

6526

6527

6528

6529

6530

653

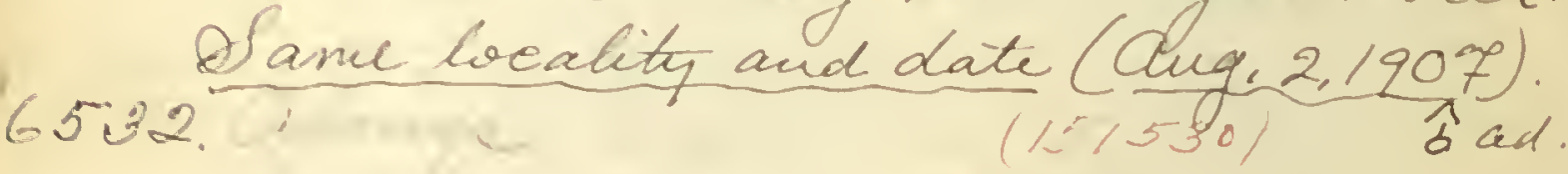
$65: 2$. Sanc locality and date (Oug.2.1907). Nate- The tere following are probable

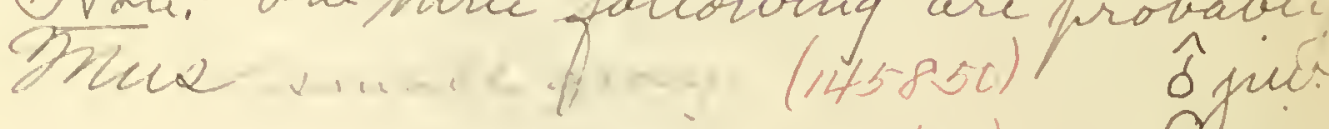
$(15,491)$ $(151492)$

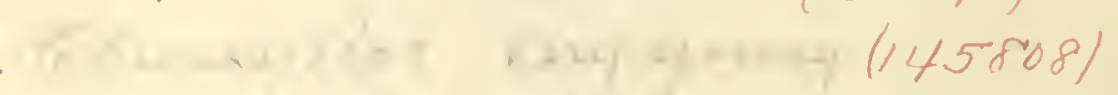

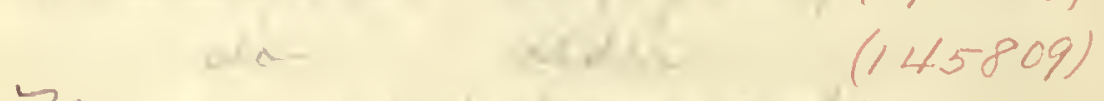
(Cossibly the rane apeice ar the two freceding.)

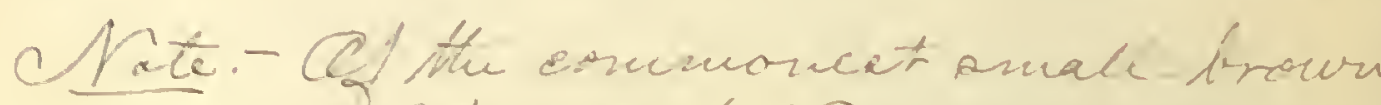
and 6 temales (Not.65-21, 6522, creve, in formatin solution, to eaclected by Mrs. Haight latcr.

$1533.27 m e$

6534

$653 \hat{0}$

6336.

6537.

6538.

6539

6540

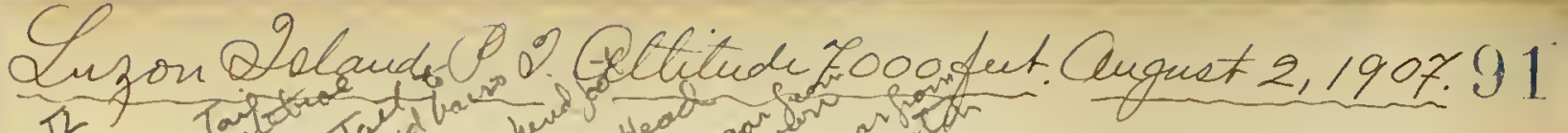
$278 ; 141 ; 144 ; 28 ; 40 ; 13 ; 18$.

$267 ; 139 ; 142 ; 28 ; 37 ; 12 ; 17$. Wanmaie $\frac{2}{2}-\frac{0}{0}-\frac{2}{2}=4$ frair $258 ; 137 ; 140 ; 27 ; 36 ; 13 ; 18$

$248 ; 125 ; 128 ; 27 ; 36 ; 12 ; 17$

$260 ; 134 ; 137 ; 27 ; 37 ; 13 ; 18$.

$250 ; 138 ; 141 ; 27 ; 35 ; 12 ; 17$

young of the above species: -

$211 ; 114 ; 26$

$199 ; 106 ; 23.5$

$255 ; 127 ; 128 ; 36 ; 40 ; 16 ; 21$

$310 ; 148 ; 149 ; 36 ; 44 ; 15 ; 20$.

$287: 144: 145 ; 39 ; 47 ; 18 ; 24$. Doubtful if the rame

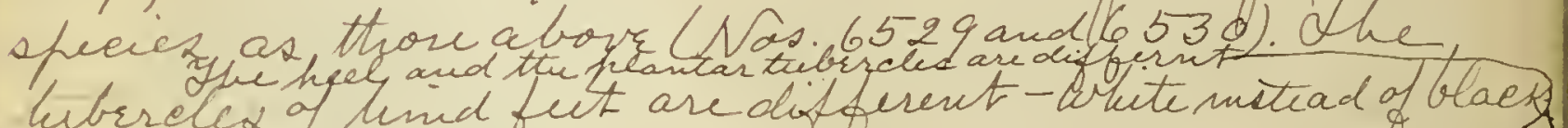
at, 6 mates (NAts. 6515, 65t16, 6517, 6519, and 65t20) 6523,6524 , and 6525) wre fut in Mh. Hanght's be forvarded to the Uesum. with othere to be

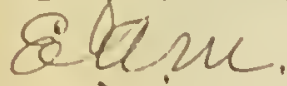

$277 ; 145 ; 147 ; 35 ; 42 ; 18 ; 21$; nose to cye, 20; to ear, 35; to tif of ear, 57; freserved in formalin. Yail defectir.

$-285 ; 146 ; 149 ; 28 ; 37 ; 13 ; 18$

$265 ; 138 ; 141 ; 27 ; 36 ; 12.5 ; 17.5$.

$270 ; 143: 146 ; 27 ; 36 ; 12 ; 17$.

Jail defectir.

$257 ; 134 ; 137 ; 26.5 ; 36.5 ; 13 ; 18$.

$135 ; 56: 58: 15: 5 ; 10$. 


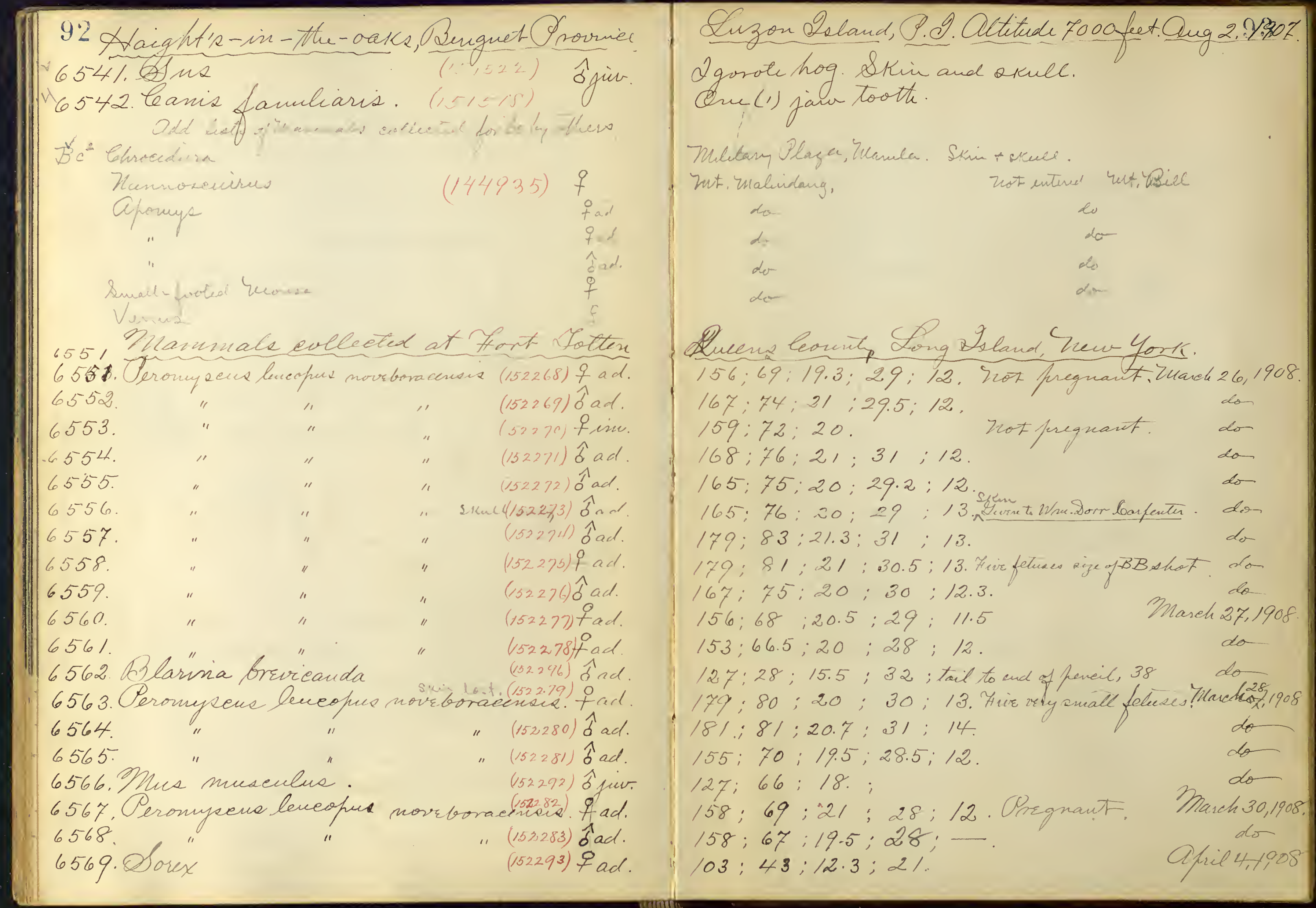


94 Mammale esclected at Fort Sotten,

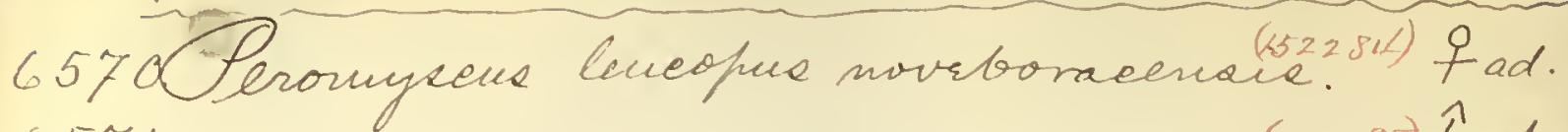
6571 .

6572 .

6573. Mne" novrgicus 6575 . Dorex

(132285) $\hat{\mathrm{b}}$ ad $657 \%$. Peromizens lencopue noveboracenciel $152288 \hat{\mathrm{b}} \mathrm{ad}$. 6578 . 6579. 6580

6581. Eorex" 6582 . Oorax

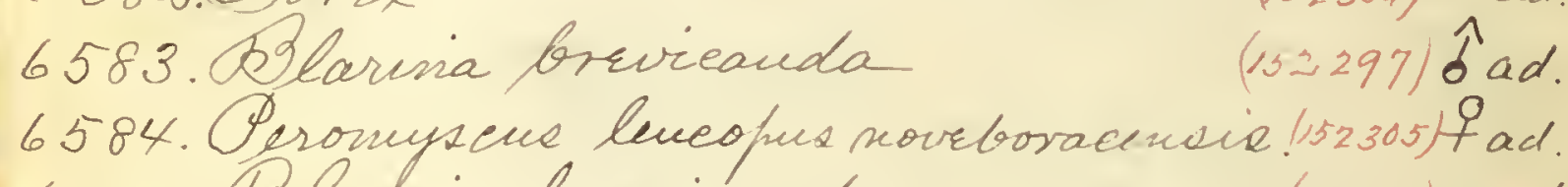
6585. Alarisia brevicauda (152307)-ad.

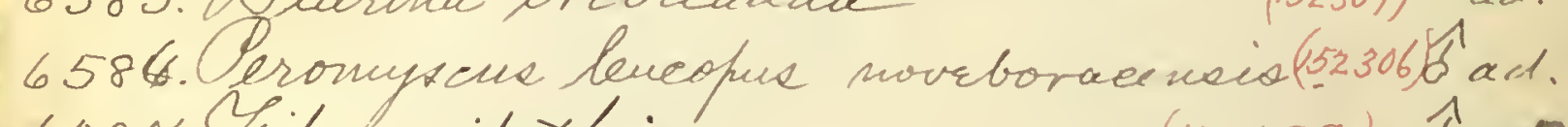
6587. Fiber zibathicia 6588 . 6589. 6590. Al"mirus carohinenis levestie 6592.143043 Terocidura

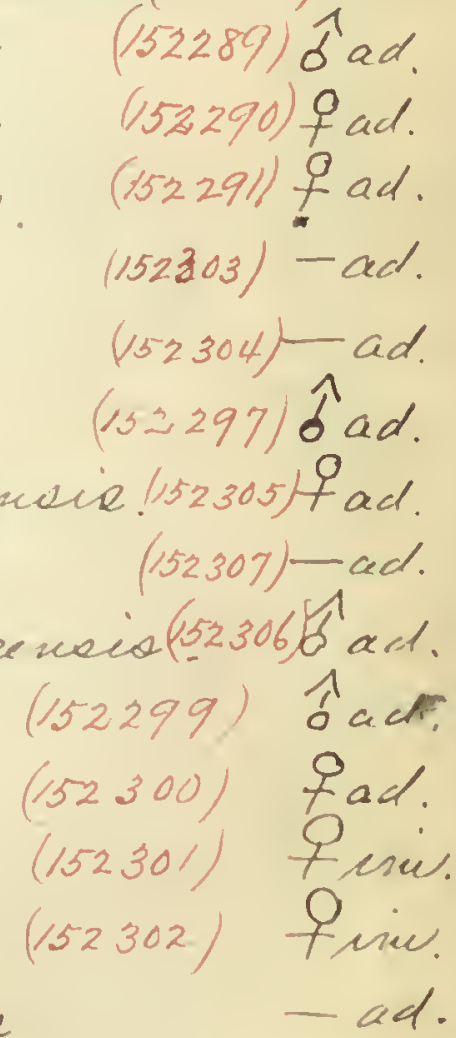

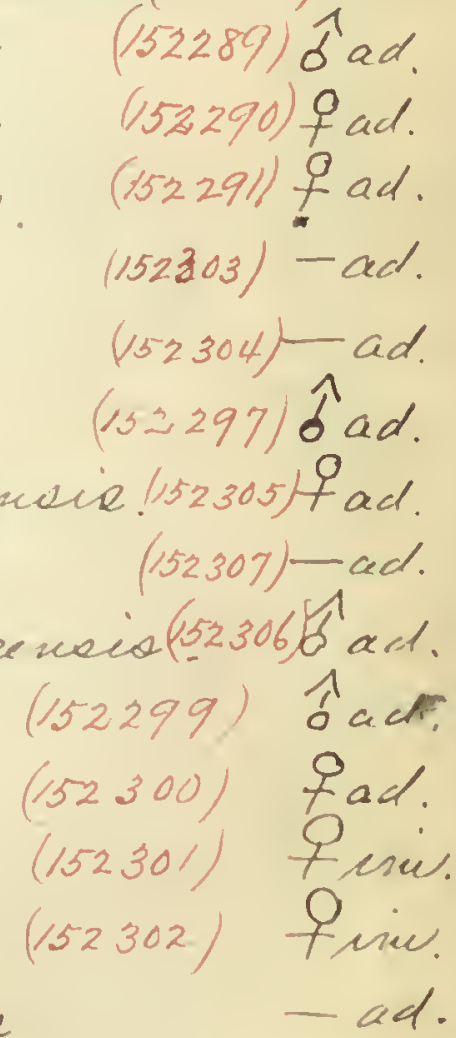

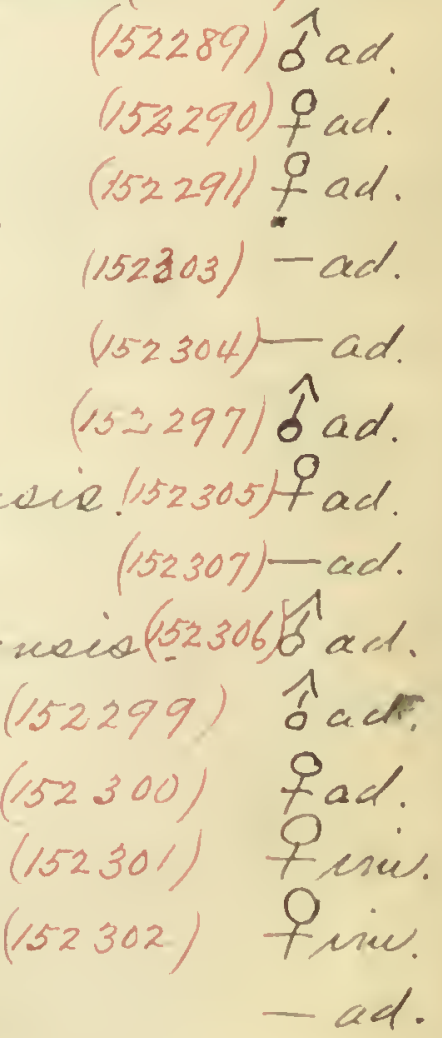

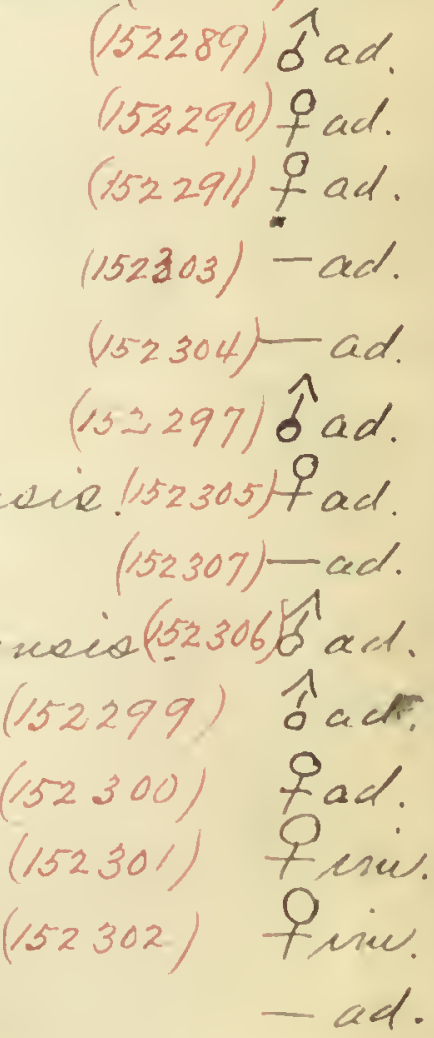

q

$6593 \quad 143044$

6594143045

$6595 / 43046$

nummal deitalo jue entinuied on haye 208 . 


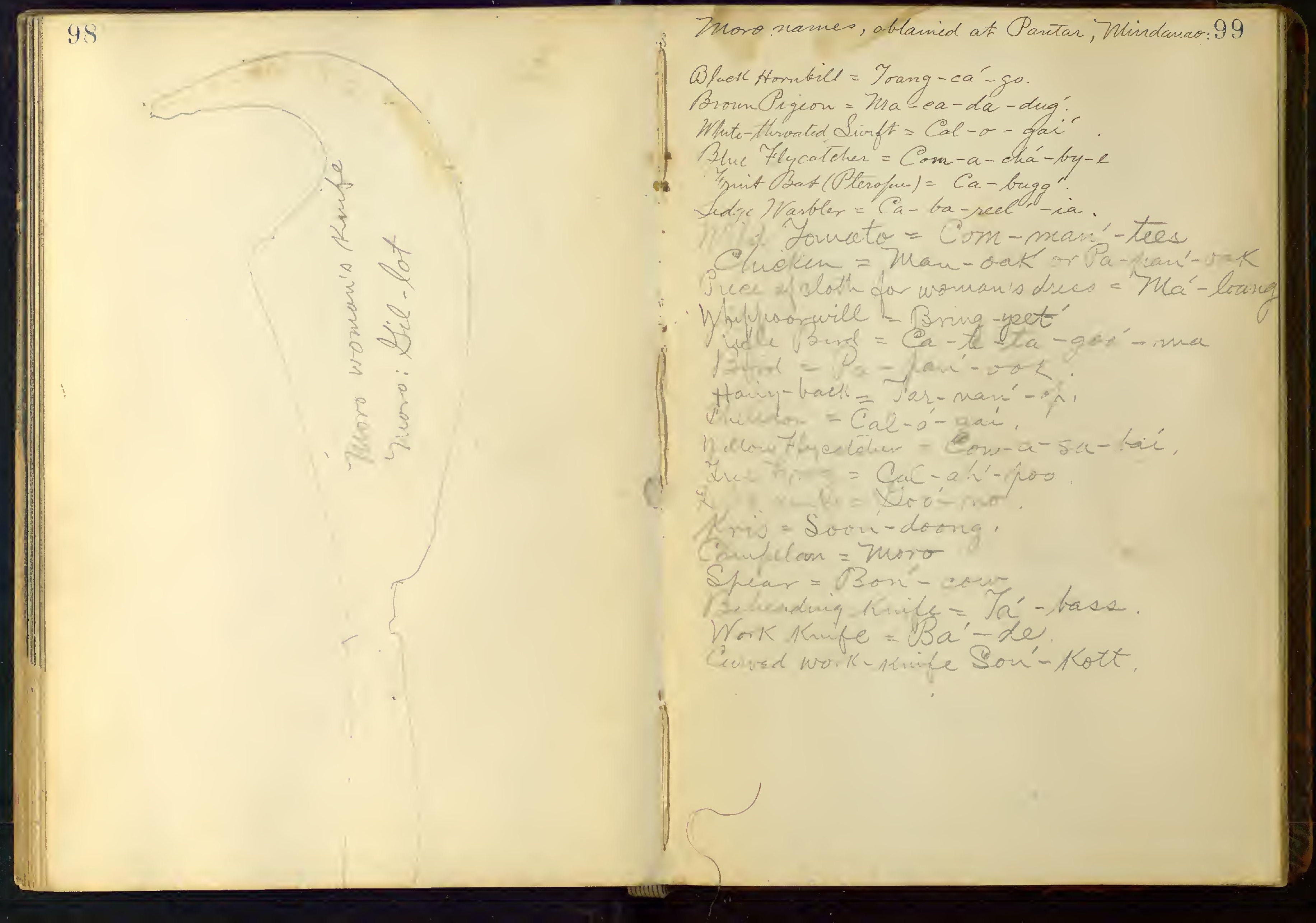


1011 Soland of Unam, Paifie Coean.

12856 Red lerupir., íad. 153:235;77;59;18;22 $12857 "$ "रad. 148; 227;77;56;19;22 12858 "llariviles tad. 135; 205; 72;5;: 16.5; 2859 Juidur or Lote 12860 Pans elegans tad. 12862 , Sray Martin. 12863

12864 12865 - Hun- yellow Narbler

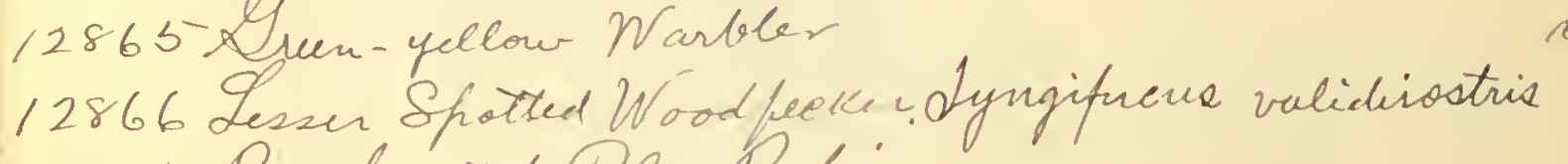

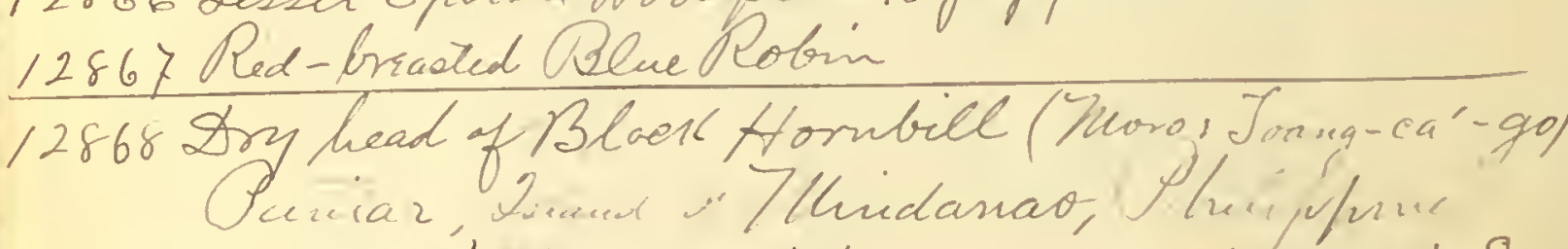

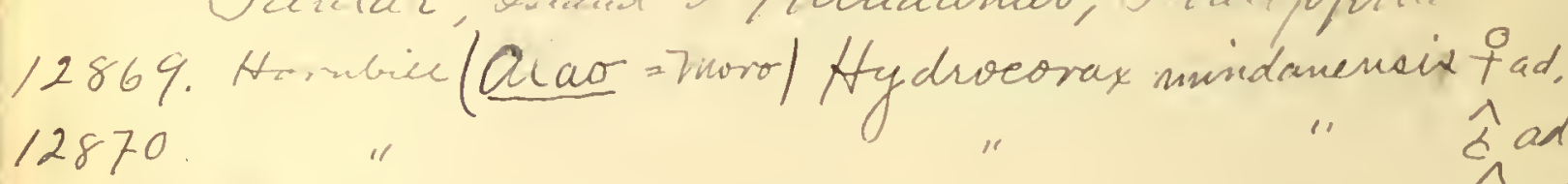

12871. Parqu" Bolbopieitacus 2873.

12874 . Brom squerov. "Mlumia fajori 12875 : 12876

12877. "12878, Roostir "enck oo. "Eintrofuce" 12879

12880

1288 Roller. Ceniptomene avinstalia tad. 12882 Broun Pegeon. Macropygia temurostris. Sad.
Ouly 21, 1909.

101

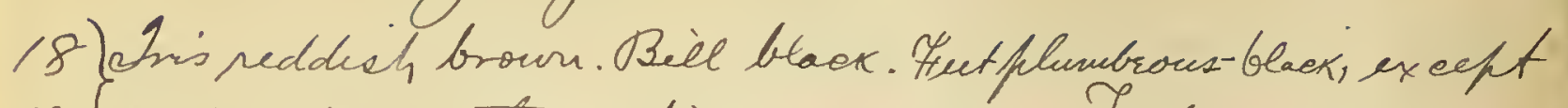

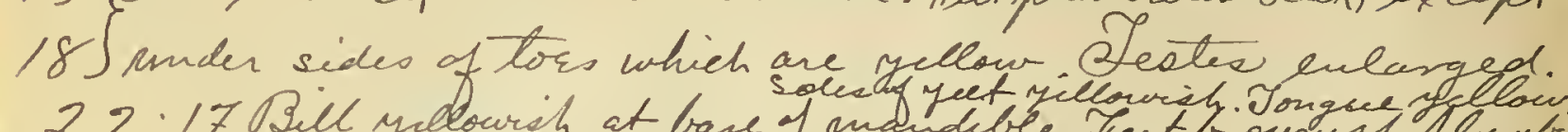

22:17. Bill yelowish at base of madndere. Fo

Word Sivallow.

do

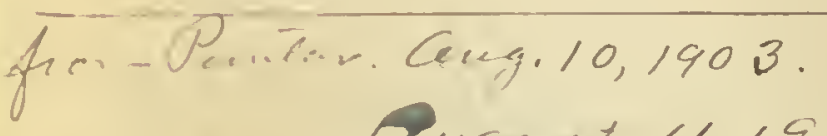

Ouguet 11,1903.

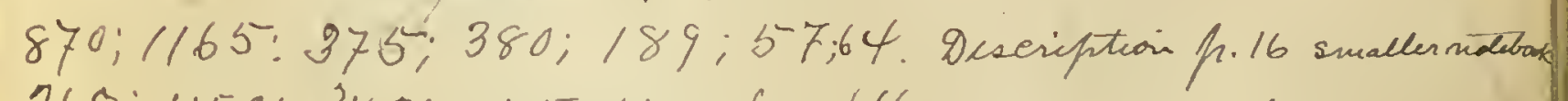
$760 ; 1150: 343 ; 275 ; 150 ; 60 ; 64$

$750 ; 1135: 336: 270: 137 ; 57 ; 64$ 


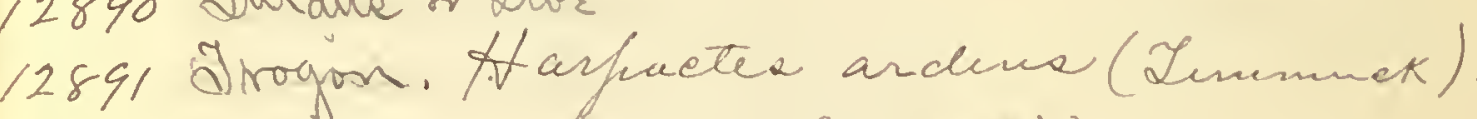

12892 ant, smuil dusk. Callocinia?

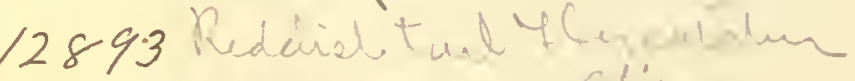

12894 tal alitio

$12896^{2}$ inrdus or 2lor

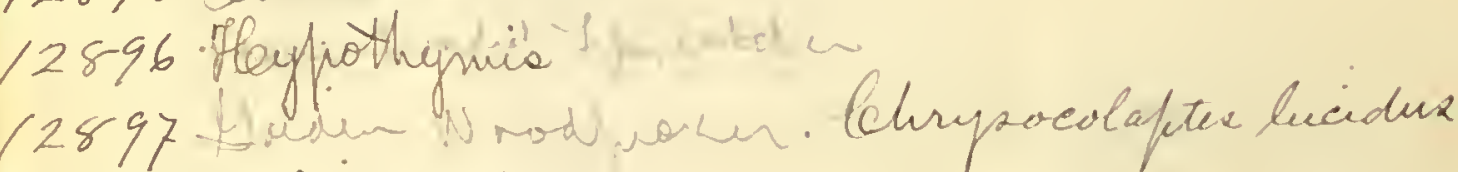
12898 Hairy-boek Mundar dussumer $P$. 
101

12911. Merops hhiliffinms

12912. "M. "Munia fagori.
12913. Black-ant-tan Spurrow "
12914 ".

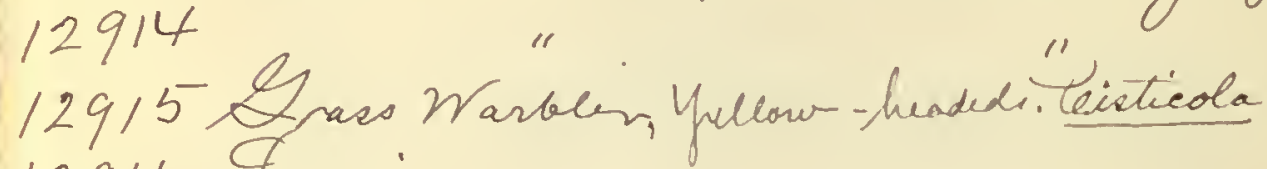

12916. Lamis

Pantar Theidanao P Q

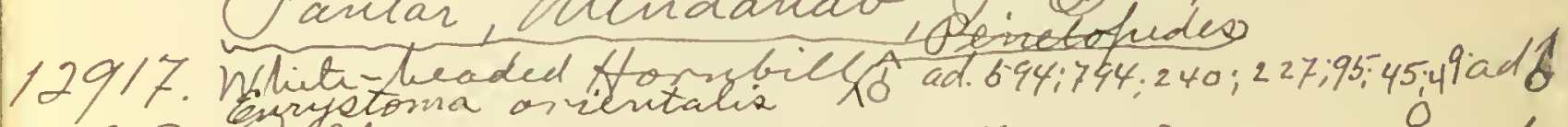

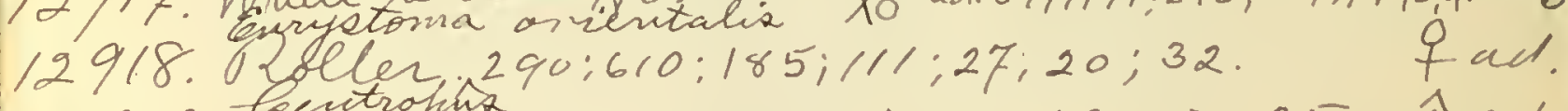
12919 Painchow $335: 420 ; 135 ; 170 ; 23: 35 ; 35$. ô ad.

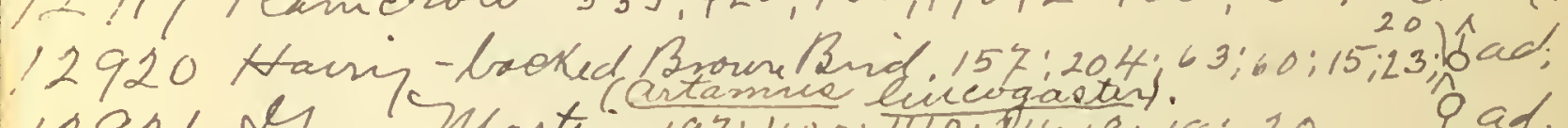

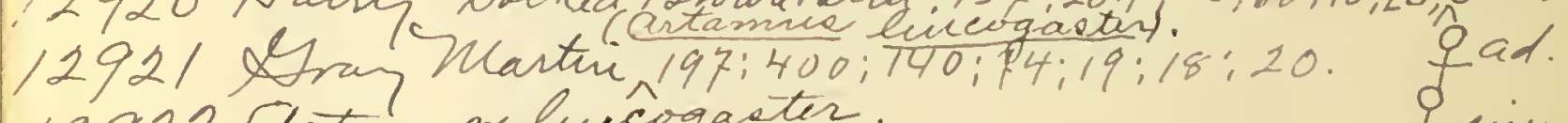
12922 Astanno luecogaster. P. P. Pyiv.

12923 . Mneropa philiphimu. Fad. 12924. Dra, Martin. Eutame leveogaster - jiv.

Pantar, Unindanao P. Q\%

12926. White-breasted Rail Smaniomian marahni. oे ad. at base. Bill afple grein. Iniciramal skin china Claws olvi-plumbrons. $318 ; 500 ; 155 ; 68 ; 38 ; 55$;

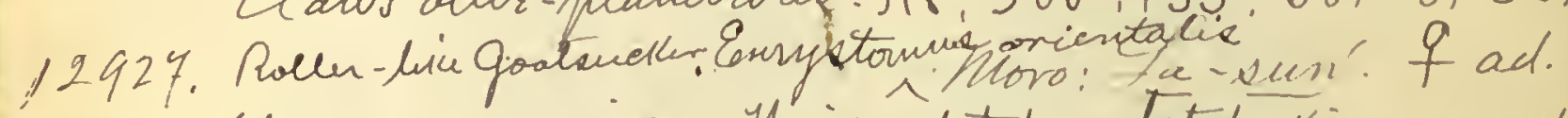
bleck on maxilla. Hheicued tubrentented skim around Claus plumbrons-blowh. 300;622;183; 112; 31; 12928. Rain-Crow. Ceentropus, Niro: Sa-goo-soak \$ad. 400,$495 ; 165 ; 210 ; 26: 41: 39 \mathrm{~mm}$. Pout of a Cantar, niendarao P.e?. 12929. Red-tailed Filycalter. 12930. Hain-back 12931.

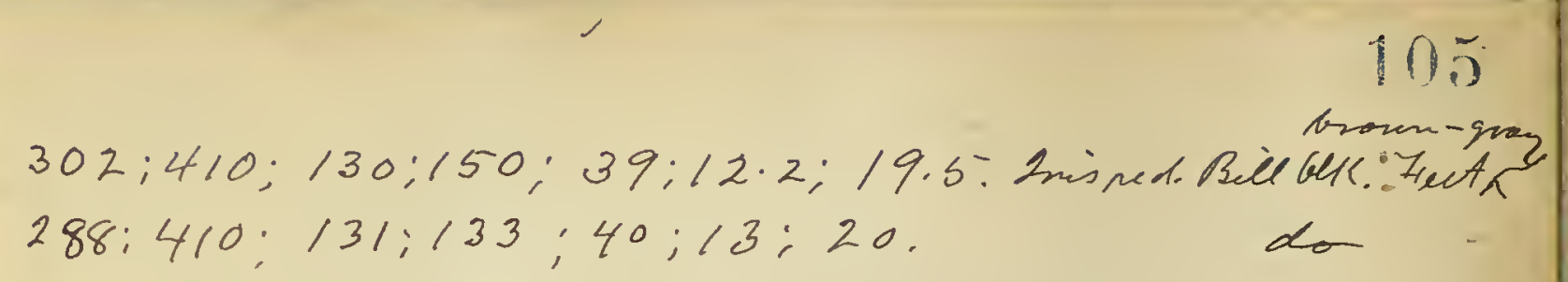

varie August 17,1903. dusk pendisan tores

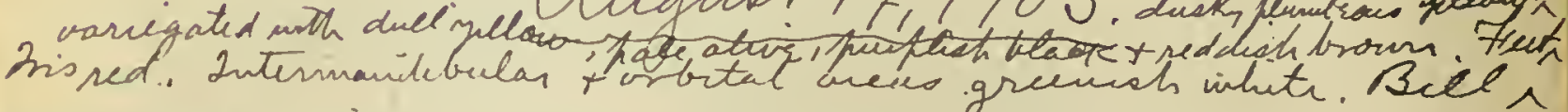

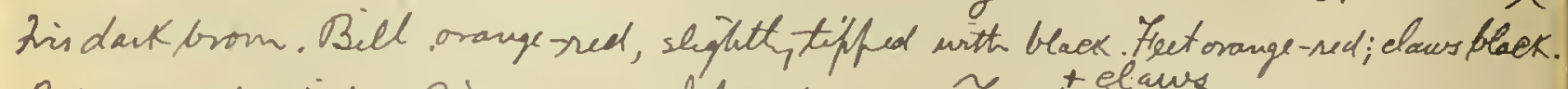

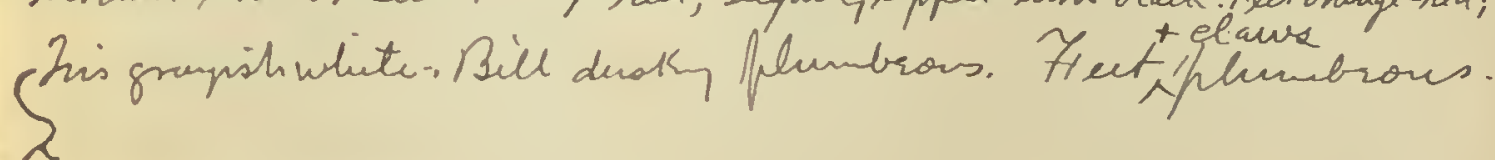

$248 ; 395 ; 127 ; 100 ; 35 ; 12 ; 19$.

$180 ; 340: 105: 44: 20 ; 15 ; 22$.

August $2 z_{c} 1903$.

Iris dauk sed. Frontiel shild osange edgee wrth yellow

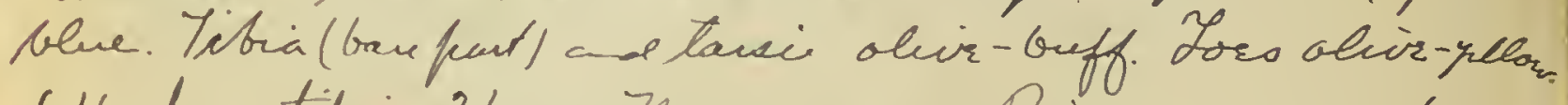
64: bare tibia. 31 inm Noro name: Bing-co-norg'.

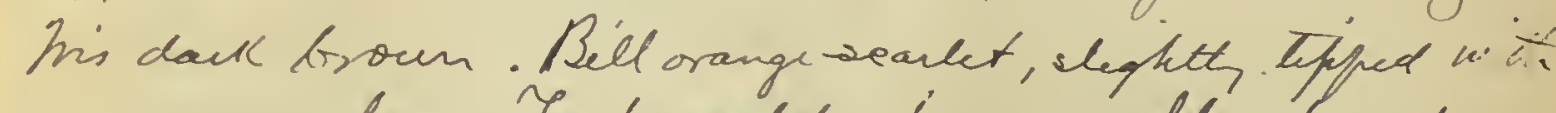
rye maracou-bry. Fect neded dragon'a blood red.

$20 ; 28$; wiath of bill, $25 \mathrm{~mm}$.

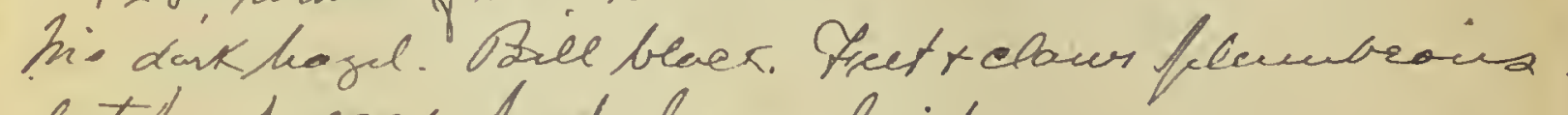
dntch of egge had bran laid.

angert $24,1903$. 
12932. Bene Kniffisher 24.8:388:106;71;43;16.5;24. $\hat{d}$ ad.

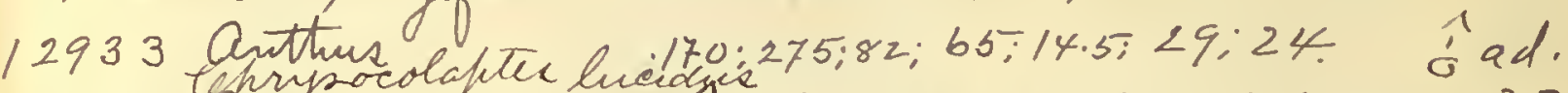
12934 . Uald Woodfecker. ó ad. 293;465;140;94; 38;28; 33 12935 . Iendus

$\hat{b}$ ad. 235:322:102:102:22.5;:5; 19 12936. Black Bind Clilua it ad. 270;437; 138:118; 30;25, 25.

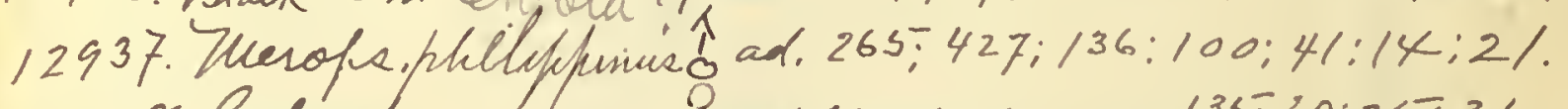
12938. Cevlumba fad.360;645;210:135;20;25:36

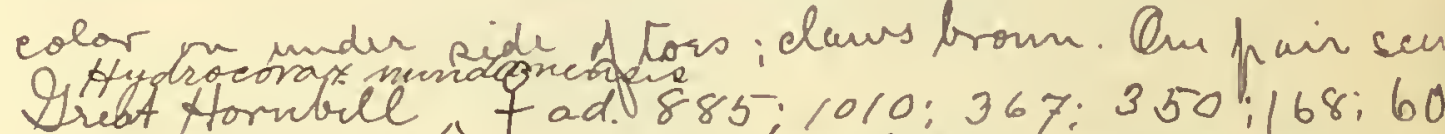
12939 . Ireat tormbll a t ad 885; 1010:367: 350;168;60 ned Claver olive Calor whitish Bill bleck 12940. Hrat tormbilun ad 920:12 30; 390;350;205: @amp Pantar, Unidanno. Halinatie $13 r$ 129.4 intit headed trawe 7 ad i.520,1350; 435, 245

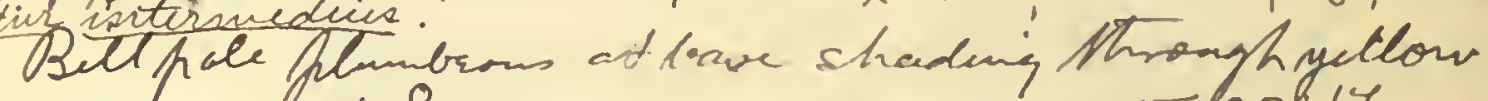
12942 Yellowbrast. + ad. $166: 265 ; 83:(-9: 15 ; 20: 1 \%$ Hreen wabler fad. Philloseopues

12944 Roed Warbler $\hat{\delta}$ ad. Eisticala

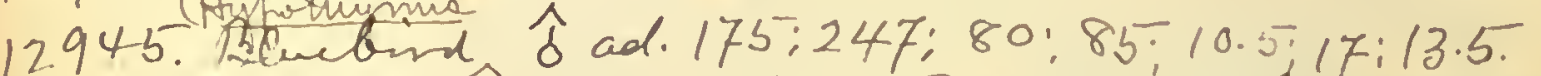

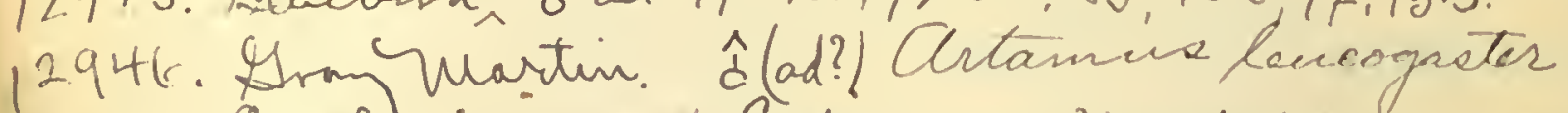

12947 Qriolus iture;! Fad. 262;368; 118;112:23;22;21

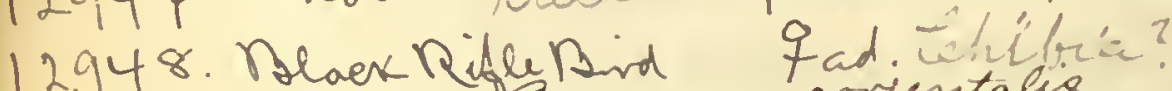

12949. Roller cursstoma oored 308 . 620: 185; 116:.28;

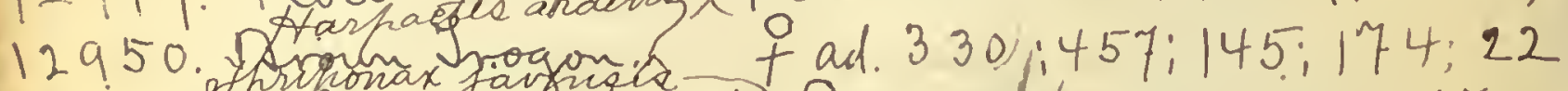

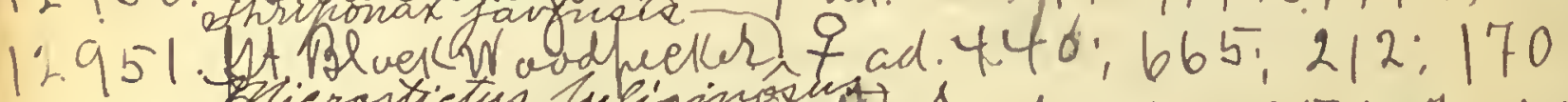

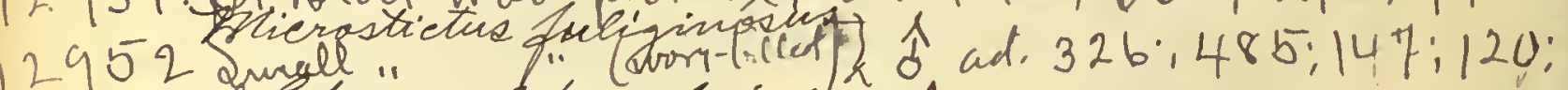

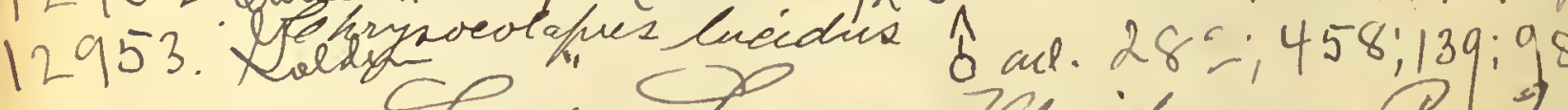

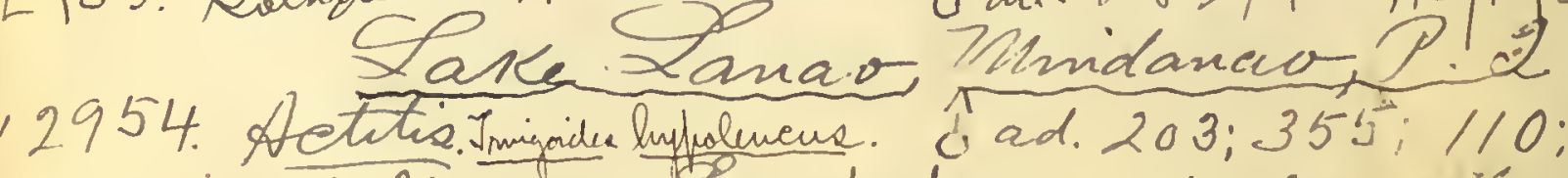

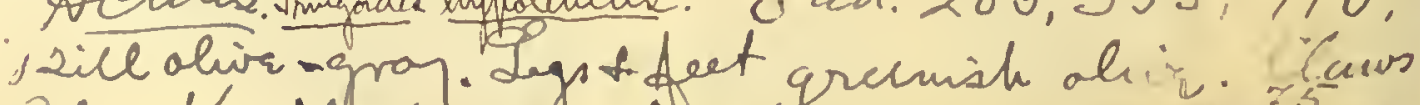

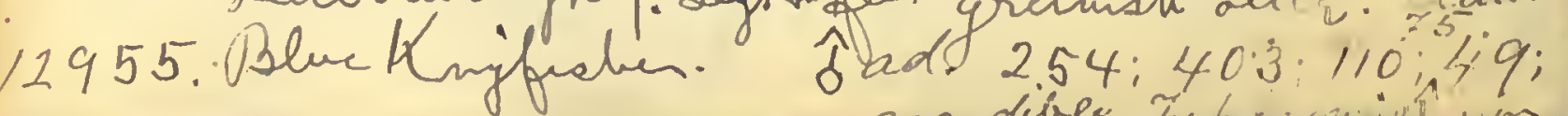

Ins white. Bill wine color tiffed inth white. Feet rnaceoces, flesh 68 i hight of bill.75. His brom. Bell blacks. Fiect dull orange 62;70. Ims Nali blue. Color redech, ete. ahminy lite). Auguat $26,1903$. 24.5; 52;46; cere, 12 . 2ni hagel. Cere hele yellow. to whit.. Fut yelow. Dews blecer. Moro = Da-pry' - O

$$
\begin{aligned}
& \text { Thoro name: Tlla-ma-ma-reeg. } \\
& \text { Pah'- loss. } \\
& \text { 7urvo mane: tro-lélu'-nah. } \\
& \text { lea-tc-fa-qootún-ma }
\end{aligned}
$$

20:31. 7Horr manc: La-sun'.

$14 ; 25$.

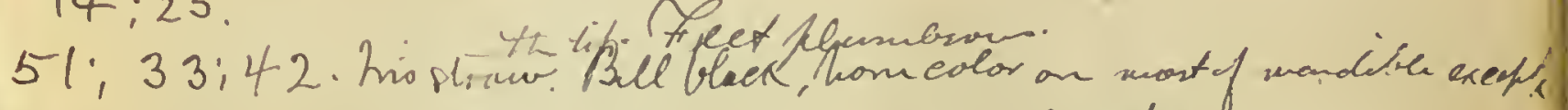
$32: 27: 34$. Ins grenich whet Bill nory. Fiect flumbarrs. $39: 26 ; 30$. Moro: Bah-la-la-took.

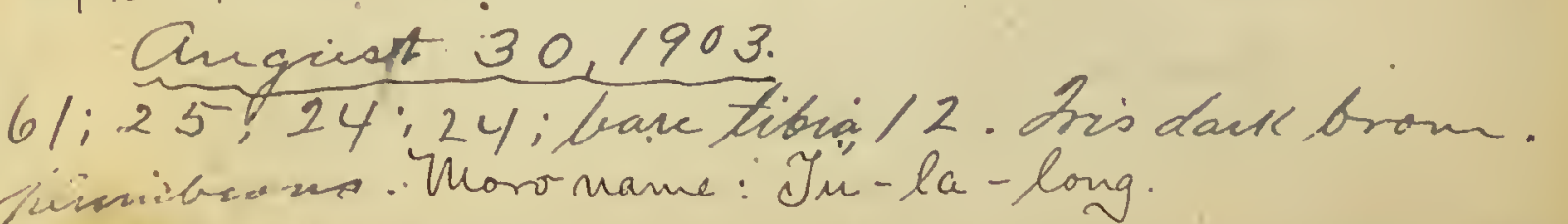

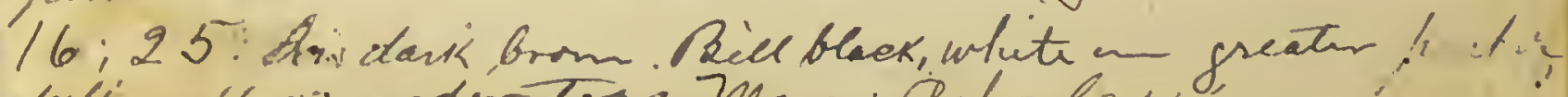


108 Gurgth to nd of clawo, 5240:390:590:174.

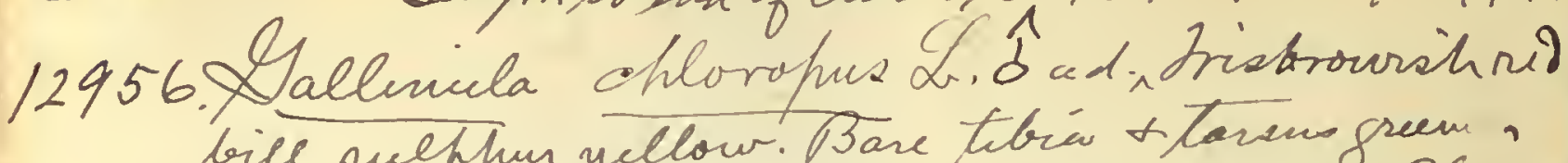

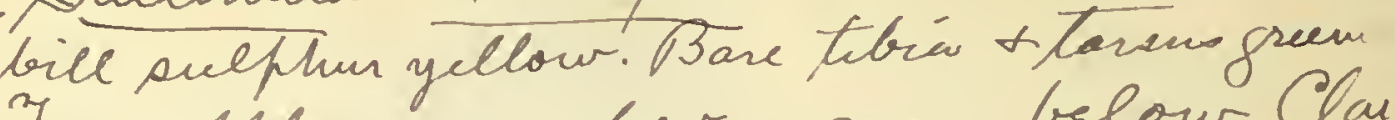

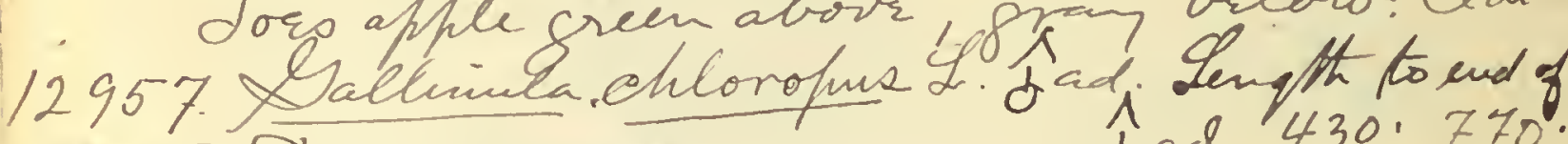
12958. Dendroegrna dod. 430; 770 ;

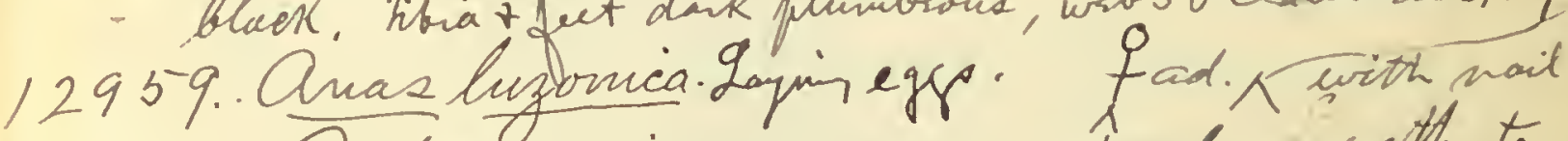
12960. Ardea cinerea bad: winth to 147;145; 153; bere tibia, 90; his yellow hingd

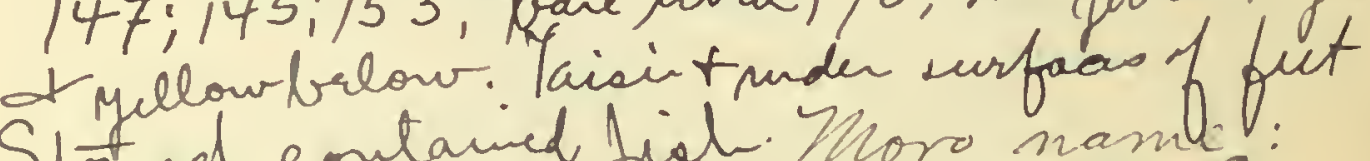
Camad equanied fiel. Thoro name?

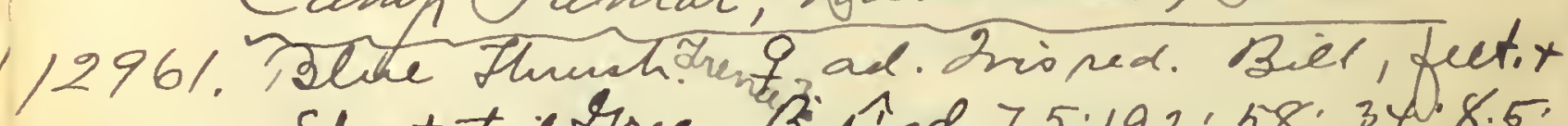

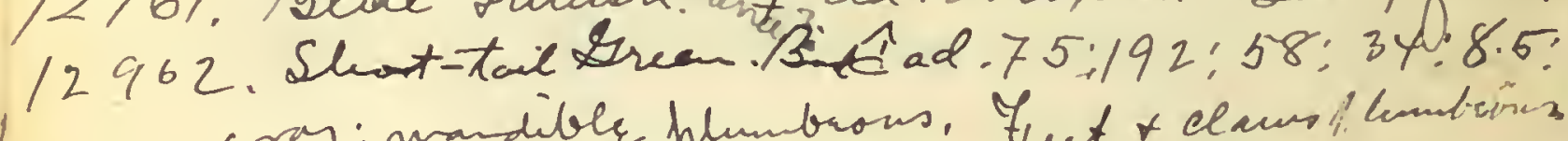

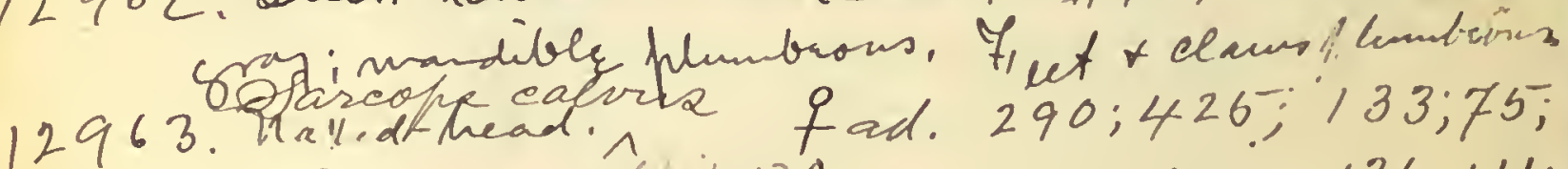
12964 . Rife Bind in $200.422,136,111$ : 12965 , of ad,264;428; 138; 116; 12966, Asmy mastin. of ad, 200:395: 135:68,

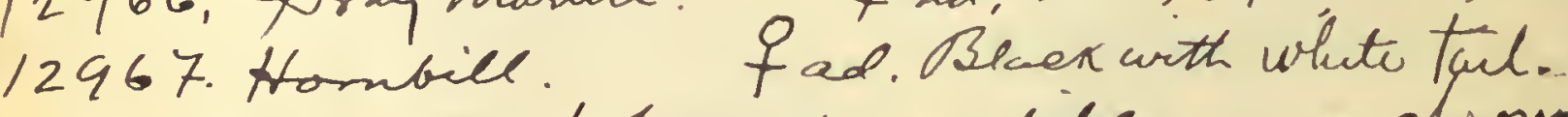
ene and at bare of mandible orange chwors

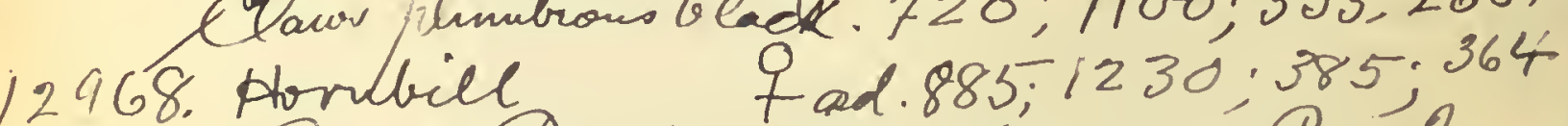
Camh Pantar, Minidanas, $P$. 9 ?

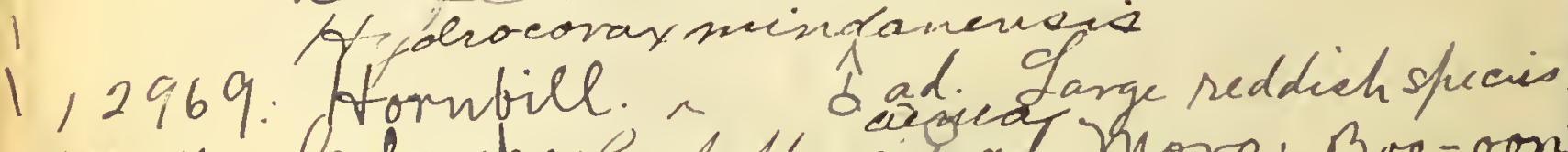

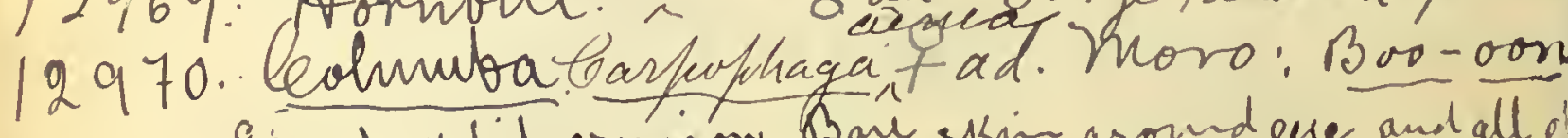

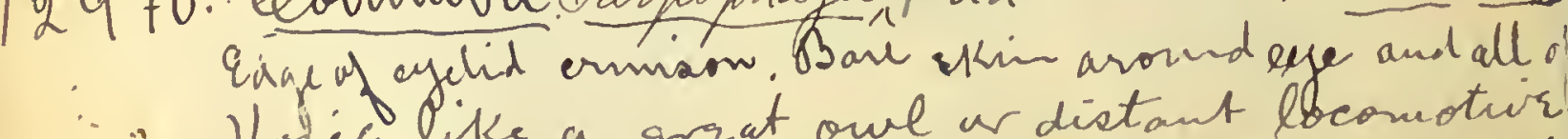

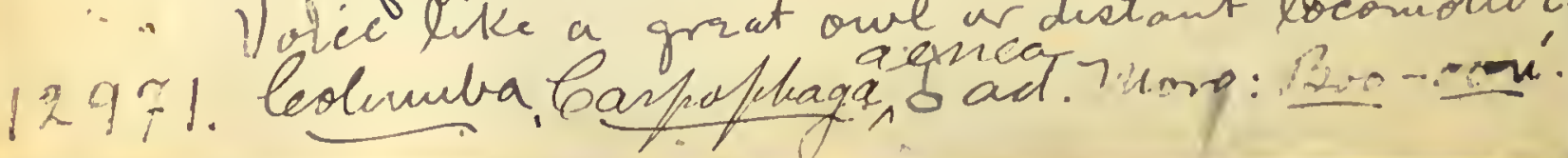

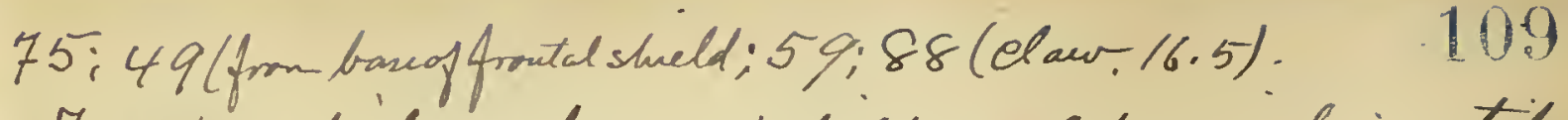
Fronter shield t base of bill searlet vasuilion; tiff of yelow, the fermer singed (meanfecter) with orange

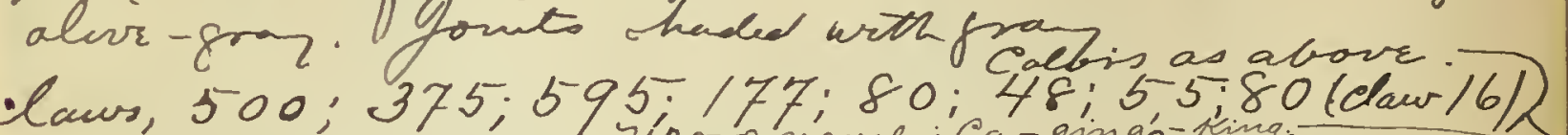

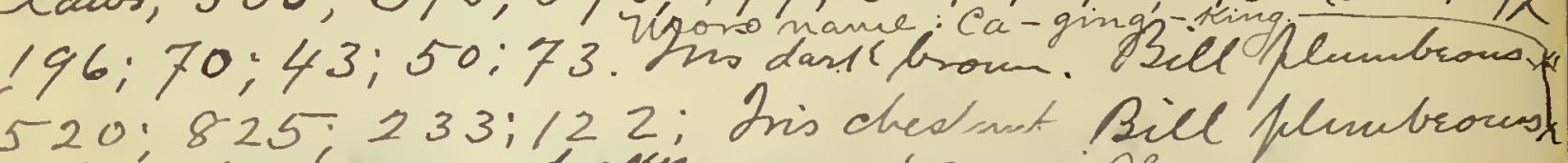

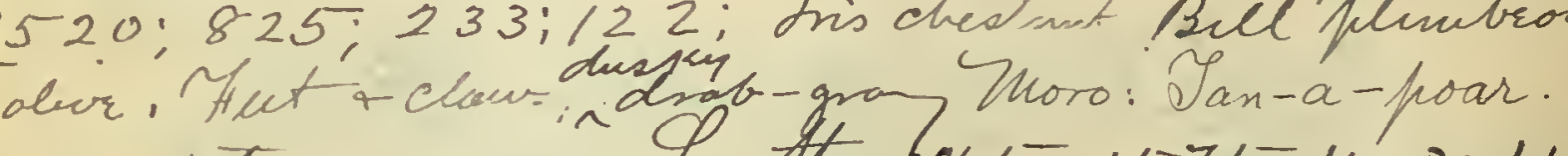
and of tos, 1450 : Le th, $1065 ; 1575 ; 400: 160$ with la mine Bare faity foer yellow. Bill alive abour

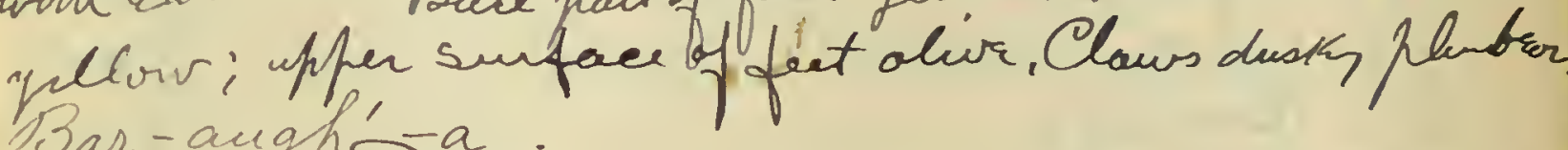
claus, black. 260:390;123:105; 26;19:24

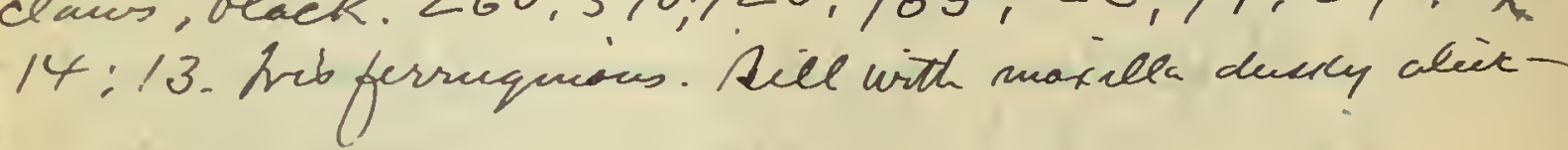
19:32:33.

$28: 21: 24$

130:22:25

$20 ; 20: 21.55$

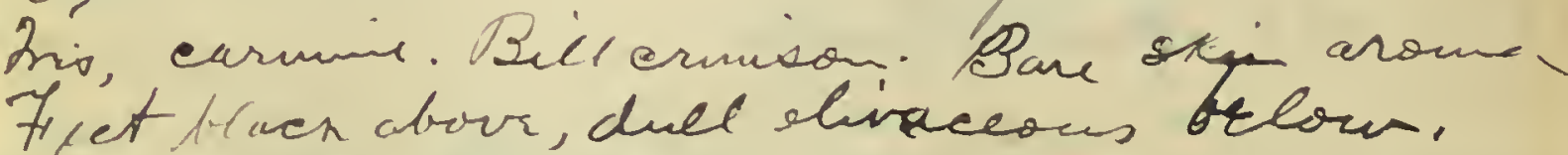
$125 ; 5-4 ; 60$. Moro: Oorang-eál go. $192 ; 62 ; 74$ Broun. Juow aC-anw'. Sept. 3,1903.

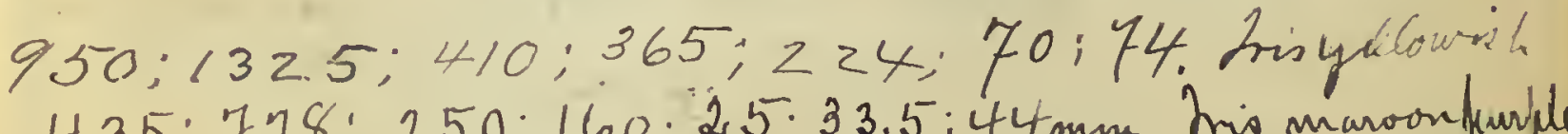

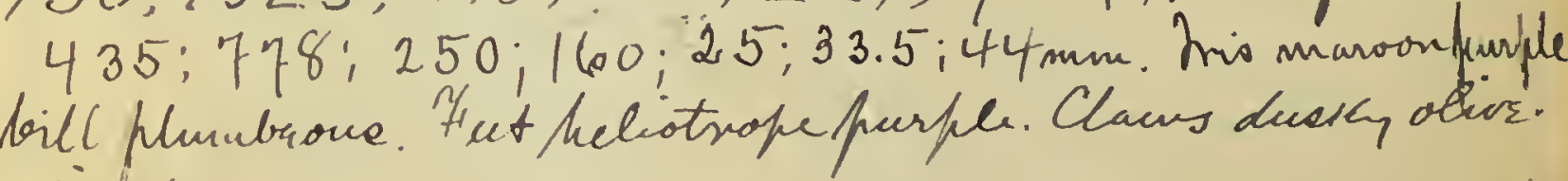
450;790:255;160:25;35;46. Colom as above. 
110 Centropus Sa-goo'soak Sad. Sualen than funale!

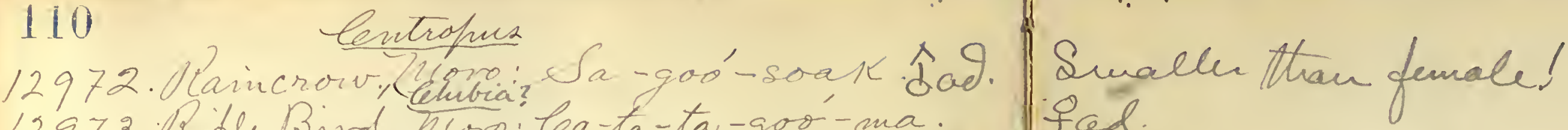
12973 . Rifle Bird Nuro: lea-ta-ta-goó-ma.

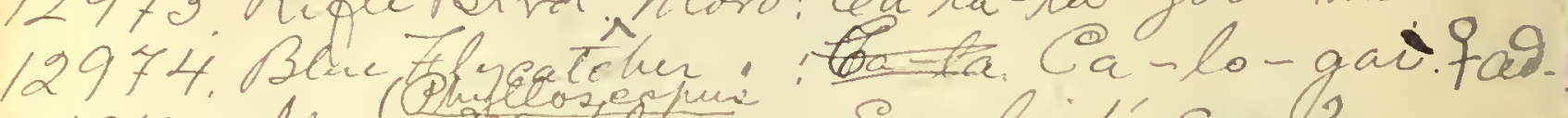

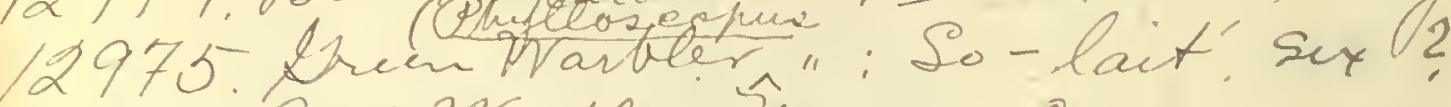
12976 . Reed Mrabler. Moro = Com - a sa-by'-e

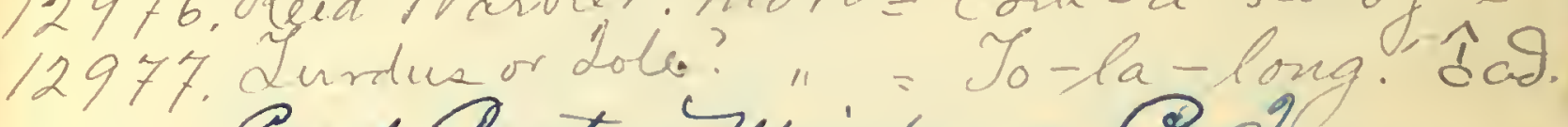

Eamp Partar, Mindanao O. ?

12978. Dove. Iurtur desenmieri (Lenum.). Tad. 12979 . Paraquet. Bolbofieitacus iad. 1.2980. DedgeWarbler. Eistie ola 12981 . Lurdus or 12982. Rain-Crow, Centropur Ŝd.

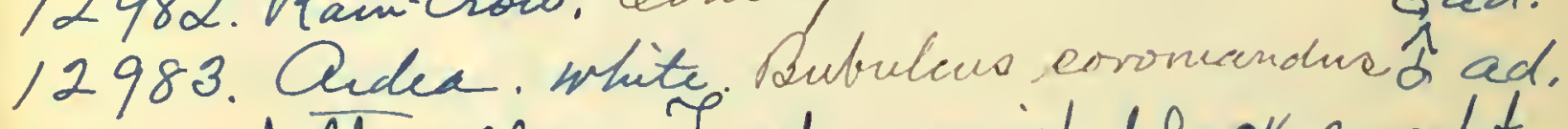
dull yollow. Fect yremish block exceft $530 ; 920 ; 252 ; 97 ; 610 ; 90 ; 77$; bere tibien 52 Cam/ Pantar, Nuidanao, P. Q.

12984. Anttura 12985 .

12986. Bene Fiencateler (Hypothyme 12987 White- Atroated Sirft 12988 "Dyenonoters goiarir (seopoli) + ad. 12989 Sulphum-vanted White headed Fily eatotien? is ad. 12990 Larger olior Crefper. Arachnothera Sad. $12991 "$ " ". 12992 Sualler Sulphur-vented Breper.

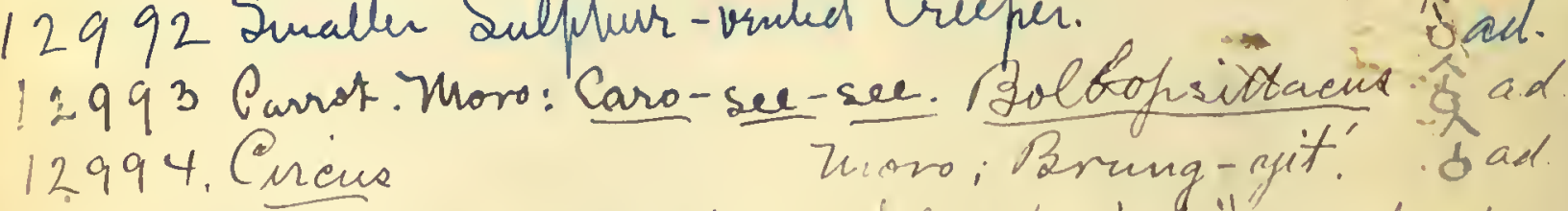
unovo; Brung-nit'. ob ad

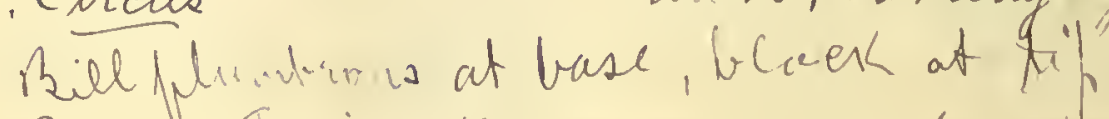

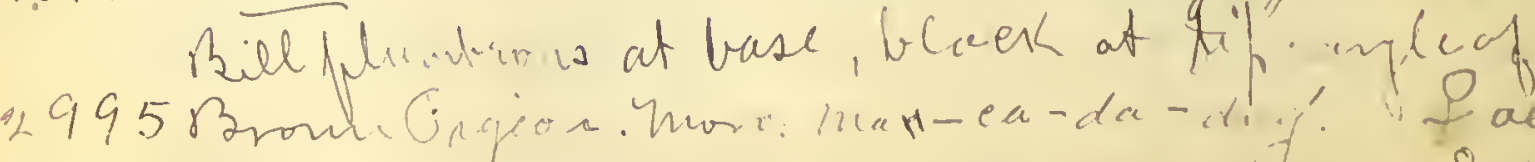
12986. Haing-linek. iad.

Hypothymie

ard. Fad leisticala

Sefit. 6, 1903.

Thoro name: Caro-see-see.

: Com -a-sa-by'-e.

Jo-la-long'.

Tris, bare skin of faci qall of bill, yellow. Li Tris, bare skin of faec qall of bill, yellow. Libia Stomnch filled with grasshopherex brittes.

Sift. 7,1903 .

$170 ; 270 ; 82 ; 65 ; 15.2 ; 28 ; 23$.

molting ball,

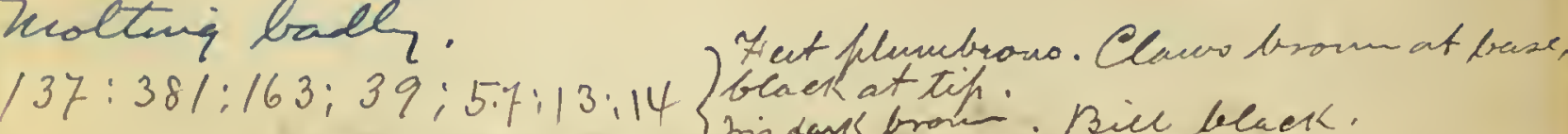

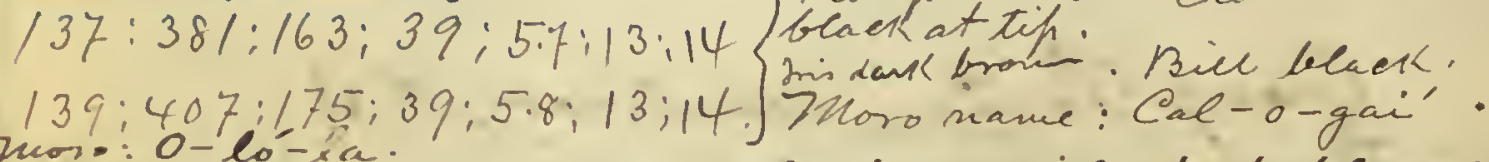

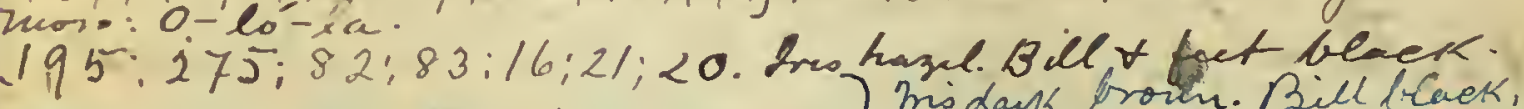
$198: 307 ; 99 ; 56: 37 ; 21 ; 21$. $194: 303 ; 95 ; 51 ; 37 ; 21: 20.3$ $136 ; 186 ; 5,7 ; 40 ; 28 ; 14 ; 16$ $180 ; 326 ; 104: 42 ; 18.5 ; 13.5 ; 24: 23$ $450 ; 1030 ; 327 ; 225 ; 15 \cdot 3 ; 70 ; 40$. Dis yellow. Cere appie grien mon th durk grem. Feet yillow. Claws plumbrous - black. $370 ; 523 ; 1$ \&8:182:14:23;30. Macropygia temirostrio. Thoro name: Yar-nan'-of. 
112 Qumf Pantar, Mendanav, Q 12997. Atains-baek 149:195;63:60;15;18;18. Fad.

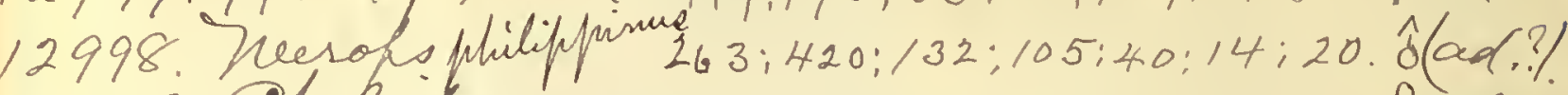

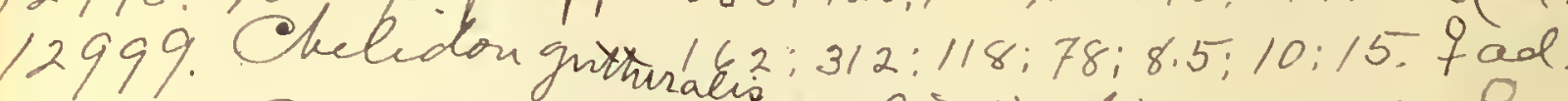
13000 . Gualomekr. noe like" nhip-poor-will t ad.

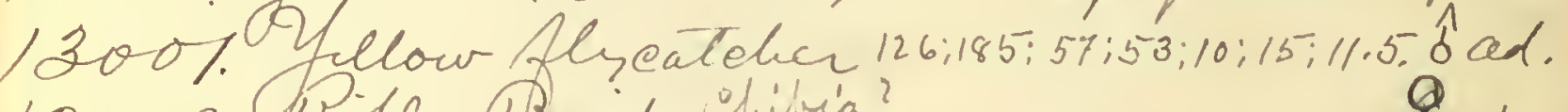
13002 . Pifle Berd Chibia? 13003

Brown Sharow hest and ot Oudar, C Pur.17,1903 Shape elogati oyate. nest fresered

13004 . Trat Bluek Yollow-brasted Noodfuaker. F ad is 13005.Ungar Mantin. Artamus lencogaeter ôn. Connfi Lantar Mindano, P. 2. 13006 . Fradu (Hanot (ned bill) Tanizunatus ô ad.

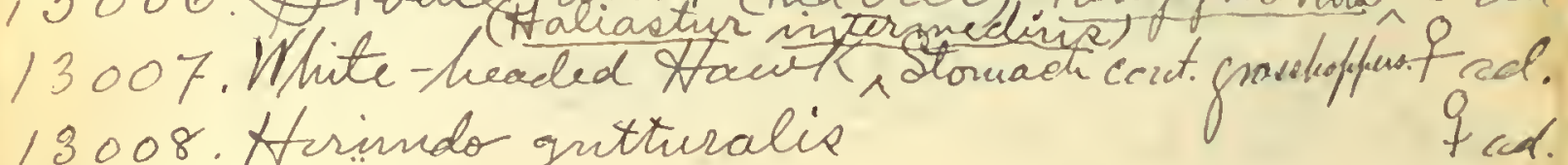
13008 . Hirindo gutturalis 13009

13010

13011 ac Caruh Pantar, Miridanat, P.

Sept.9,1903.

113

Zleor name: Dor-man'-ofe. Iis red:

$272: 547: 212 ; 1,26: 24.5 ; 19: 25$

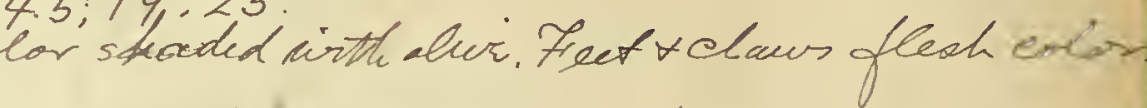
$263: 420 ; 140: 118: 29 ; 20 ; 23$ sum.

verollecy bad?.

Gas 4, white, mearuring $17 \times 11.5 ; 17 \times 11.7 ; 16.3 \times 11.2 ; 16.2 \times 11.3$

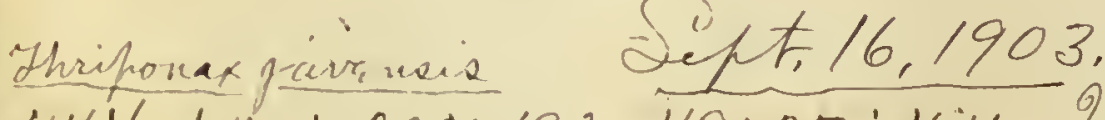

$444 ; 650 ; 220 ; 193 ; 49 ; 35 ; 4 \%$. nis straw calor. $204 ; 102: 75 ; 21: 19: \frac{\text { Seft. } 19.1903}{20.5}$

$328: 600: \quad 196: 123 ; 35:$ deptheil, $38 ; 02$ $545 ; 1.380 ; 420 ; 245$; antece, $96 ;$ cul. $26 ; 60 ; 50$.

Muiled at me shot an tefthone wire

Seft. 22,1903.

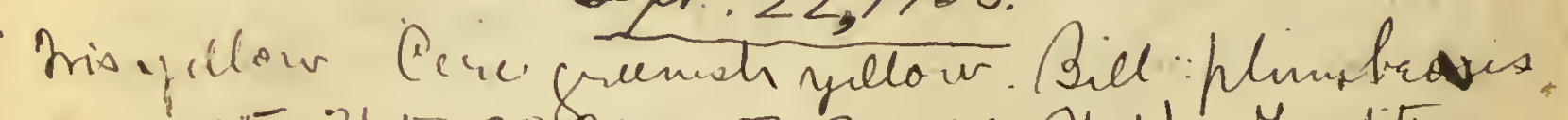
$510 ; 1145 ; 365 \cdot 23{ }_{3} ; 17.5 ; 80 ; 48$. Shot fy Mrmatita $940: 1235: 330 ; 265 ; 84 ; 45 ; 80$. Jris somen sice

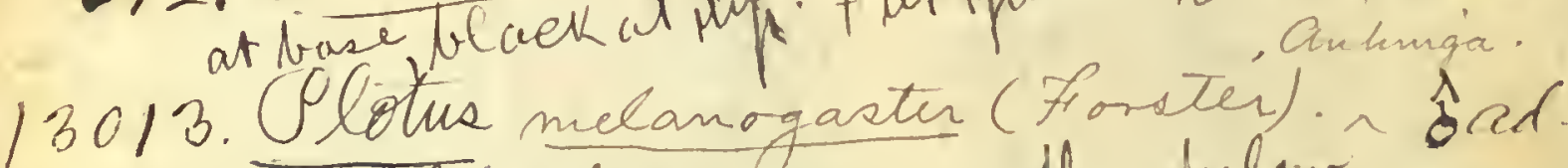

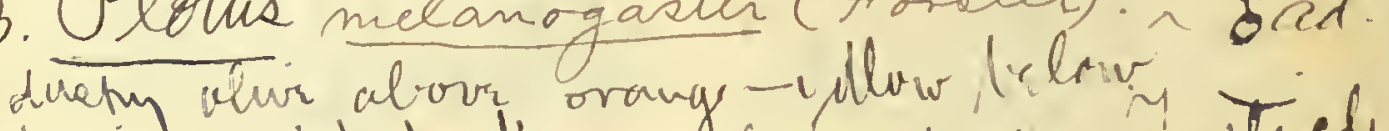

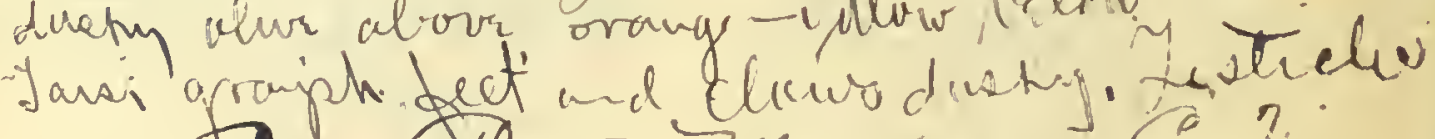

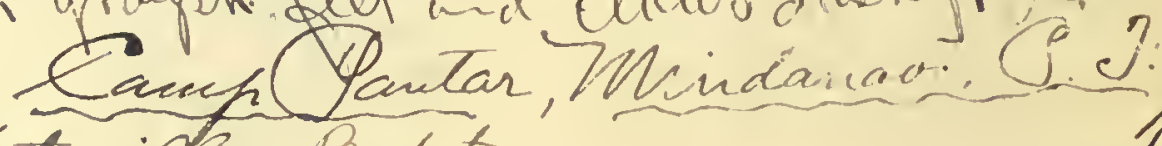
13014. Motacilla Budytes $130 / 5$

13016 . Anttine
Sod.
o ad.
$\hat{s}$ ad.

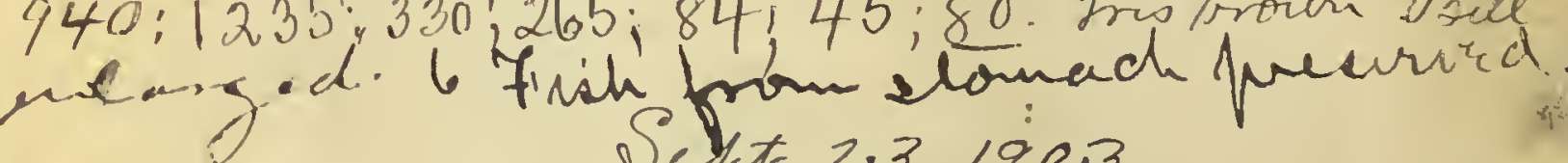

Seltet. 23,1903 .

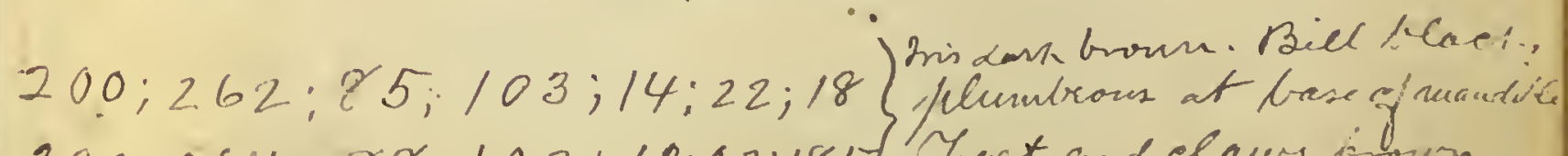

$200 ; 264: 88 ; 102 ; 13.22: 18.5\}^{\prime}$ Frat and elaws is sem $175 ; 274: 84 ; 107 ; 14.5 ; 29 ; 23$ 


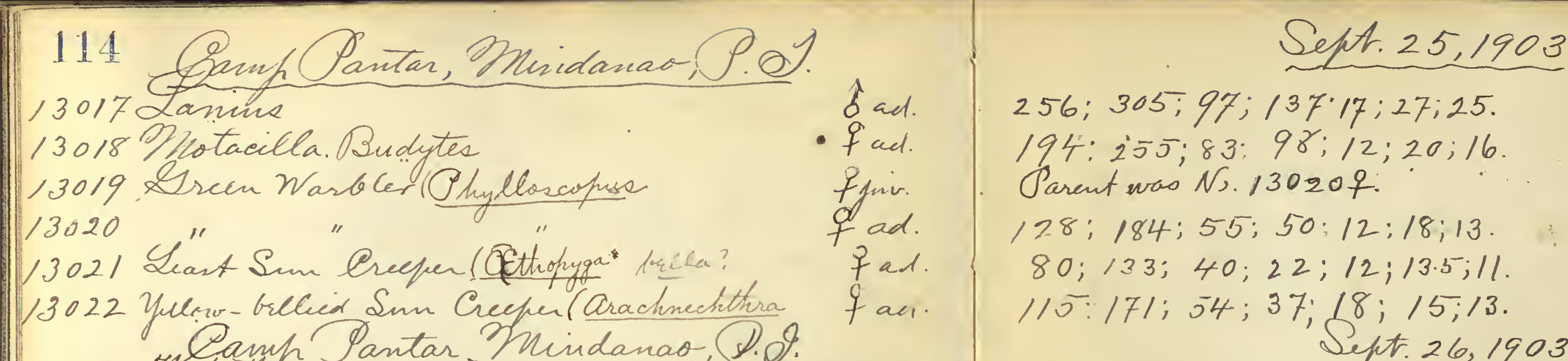

115

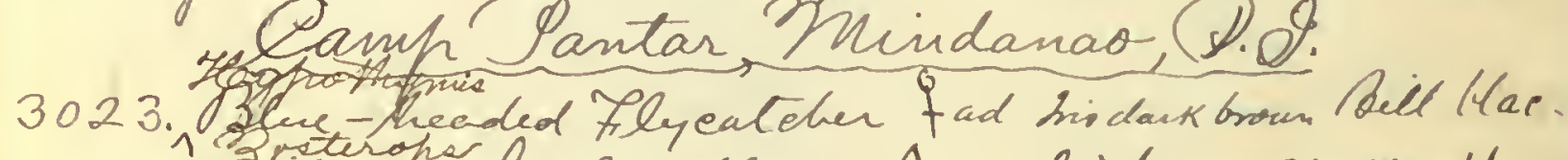

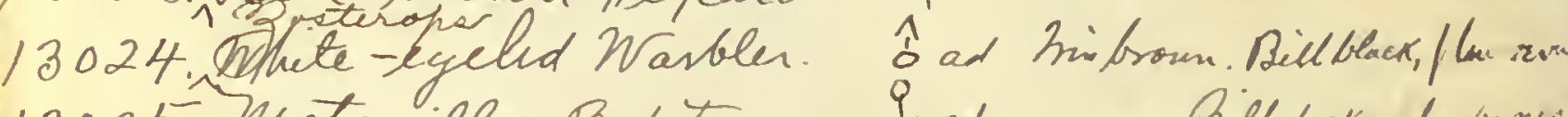

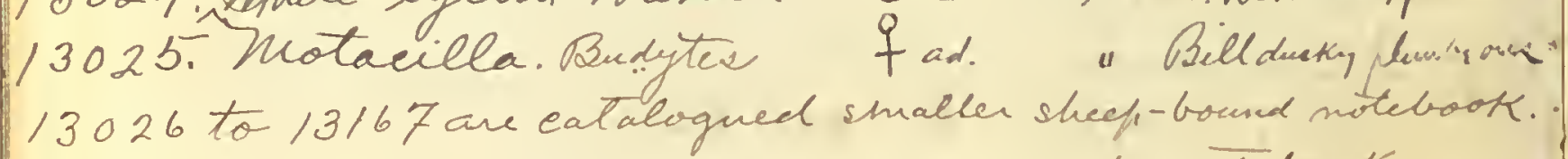
13168 to 13323
Heetblue. Hypothymis

ept $26,1903$. at brabe of numadite. Frect flemetrona. $117 ; 181 ; 56 ; 45 ; 11 ; 15 ; 12$.

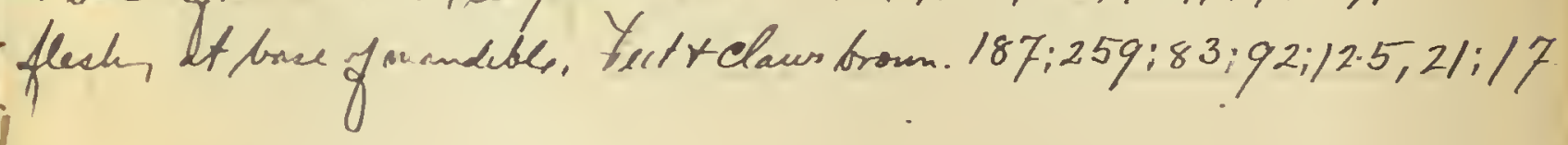
13324 ta 13436 .

Bids collecled on the Agusan Privir

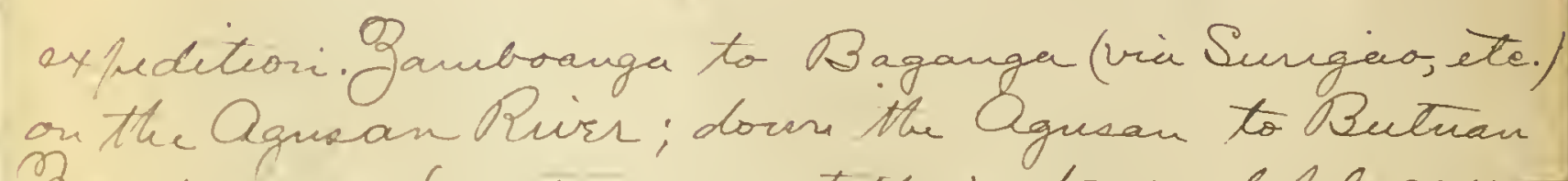
F at the mouth of that Rivin; thereer wast to [343\%. Dlosurs melamogaster (frowter). Aphriga ad.] $[3438$. Mniafra.

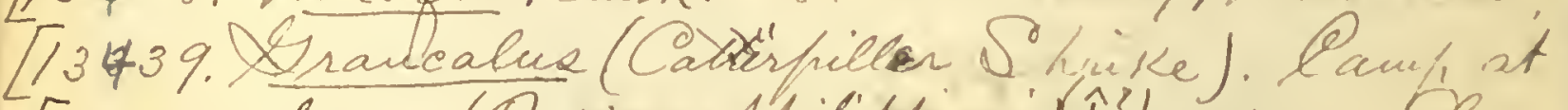

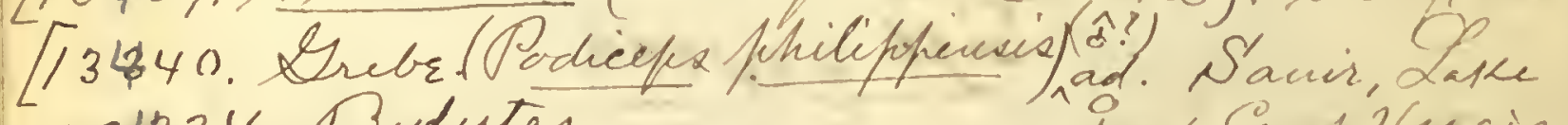
13424 . Budytes

13425

13426. Lark" "Nhirafra

13427.

13428 . Drail

13429. Suace Aedge Warbler Cisticola F̂ad. 1330. Pail-Prake. Lallierex cinerea. Sad

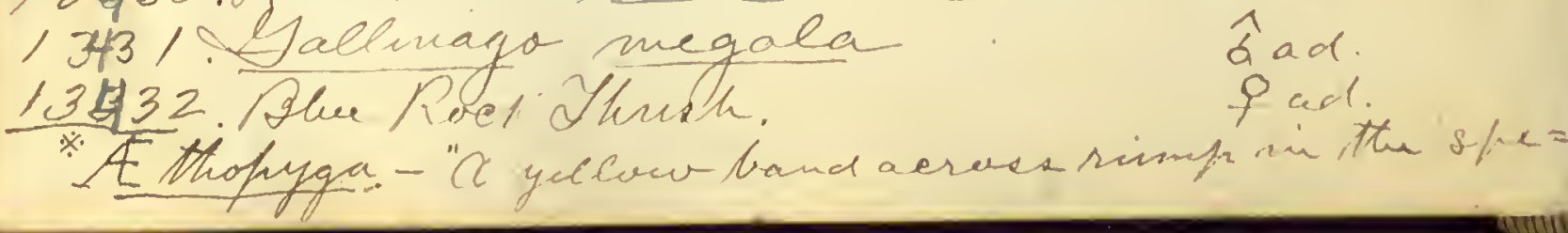

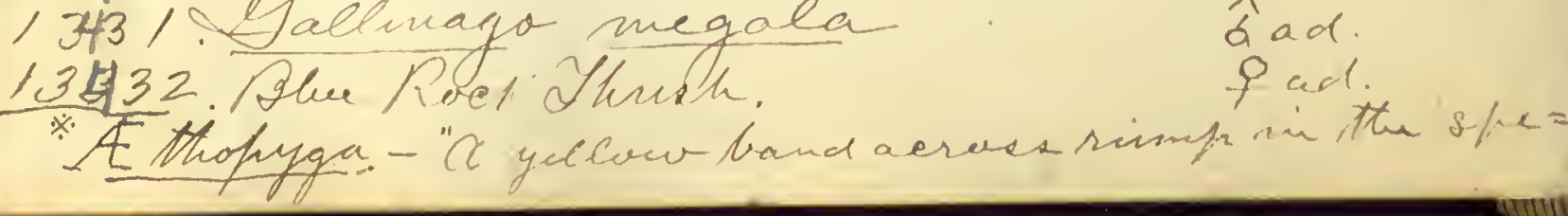

P. 9. Qiclobr 7.1903. On grassy läget range.]

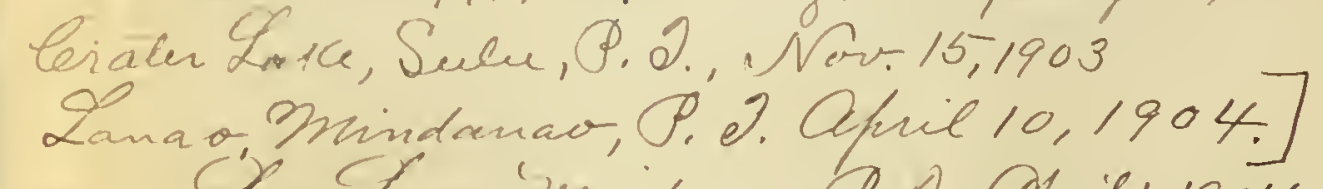

fad.

p

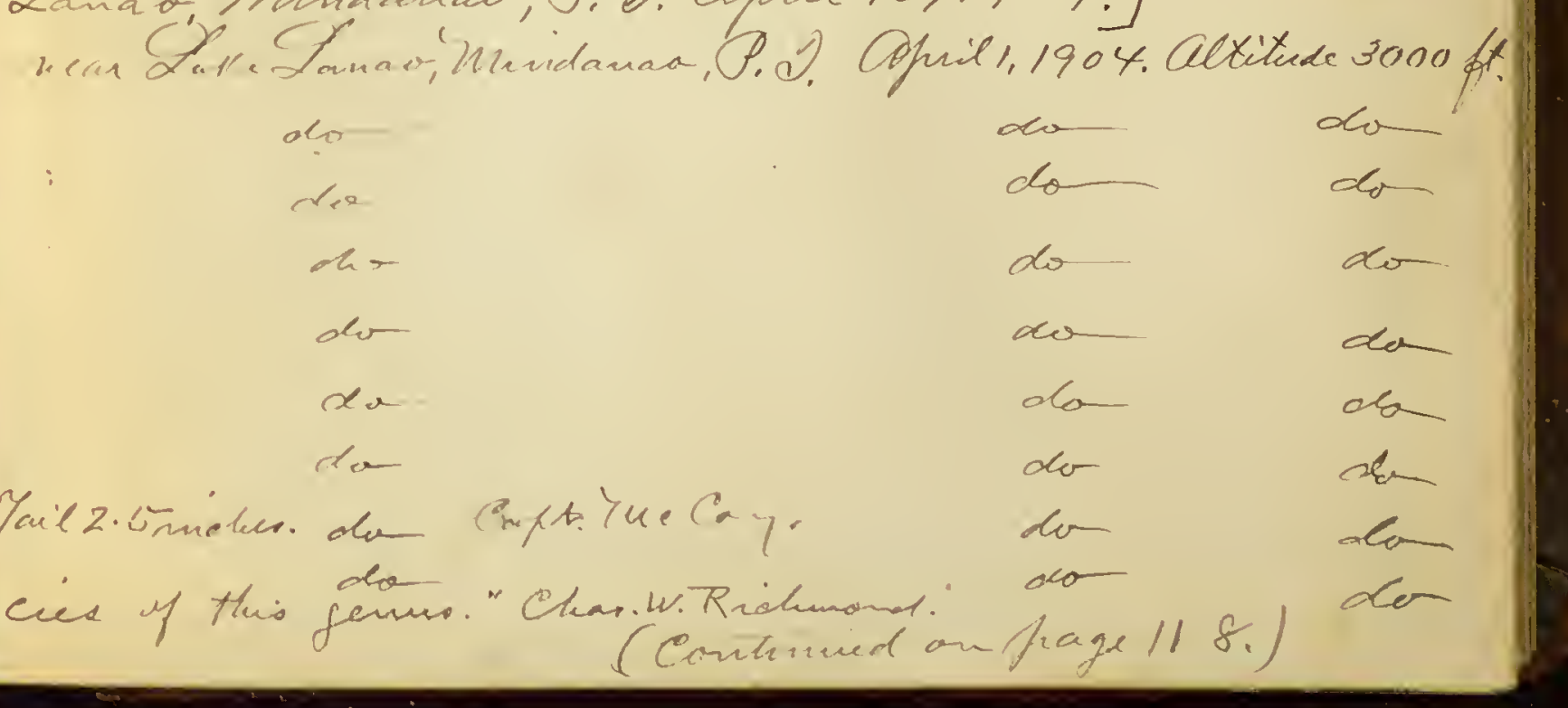




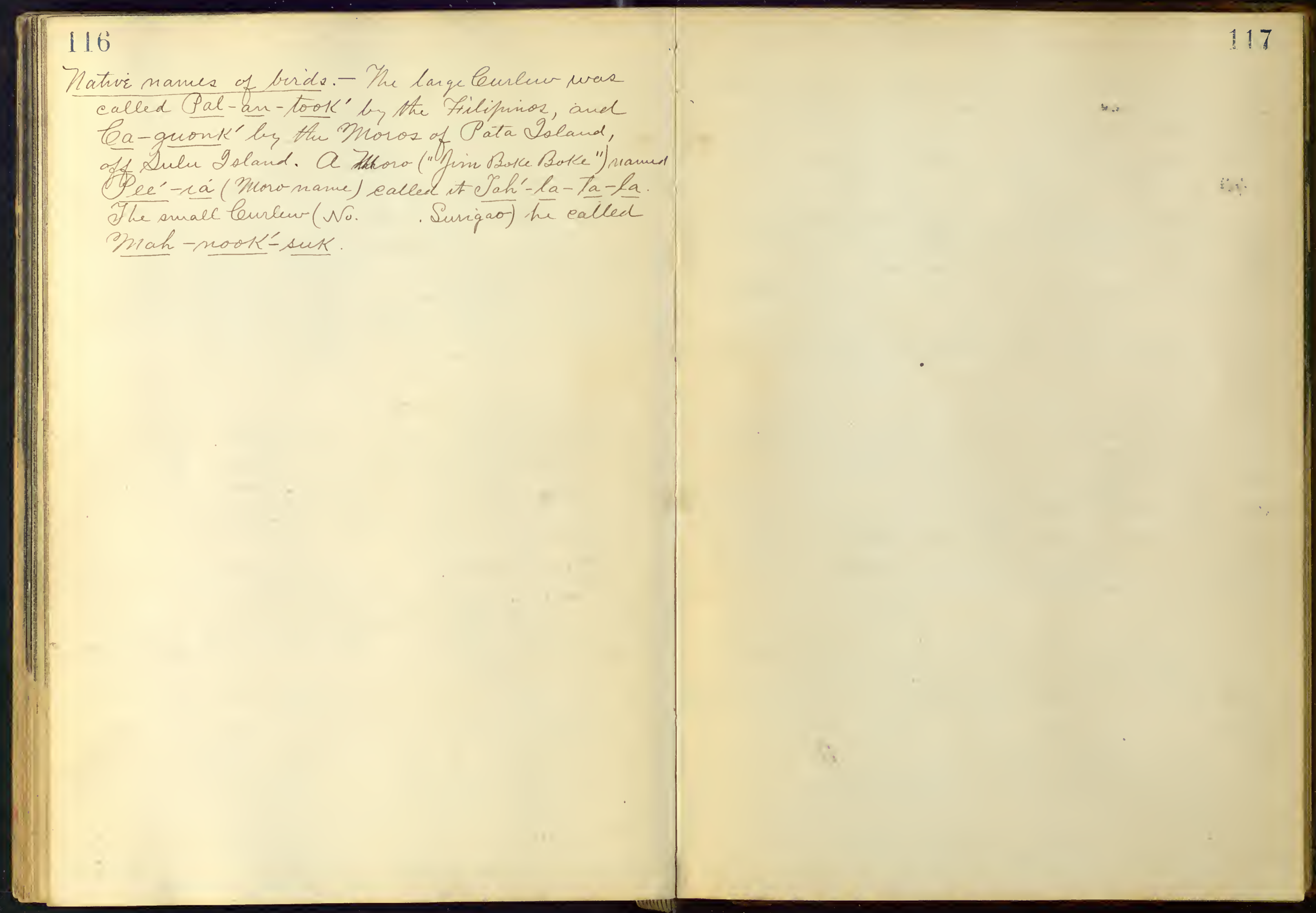




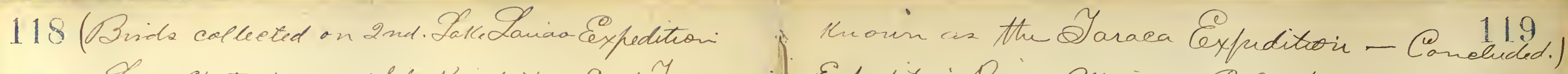

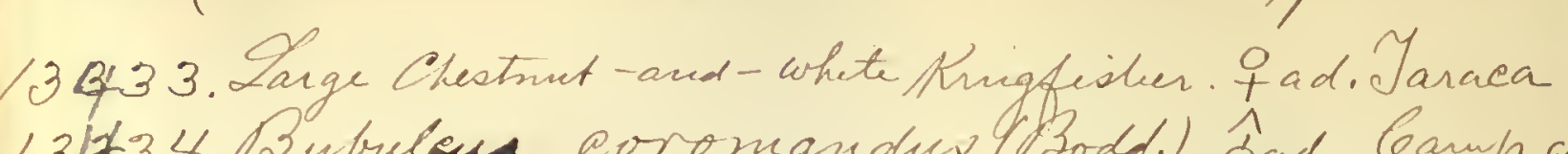

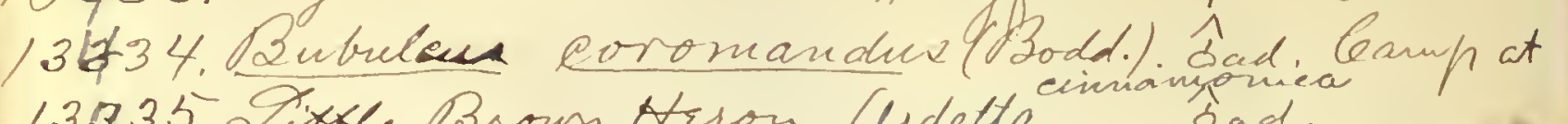

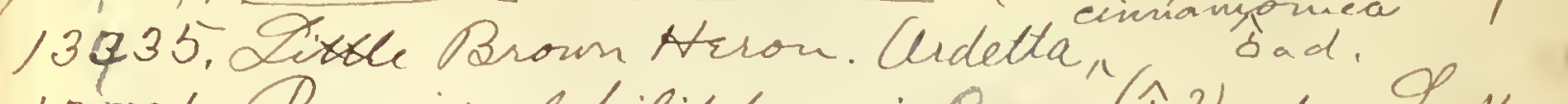

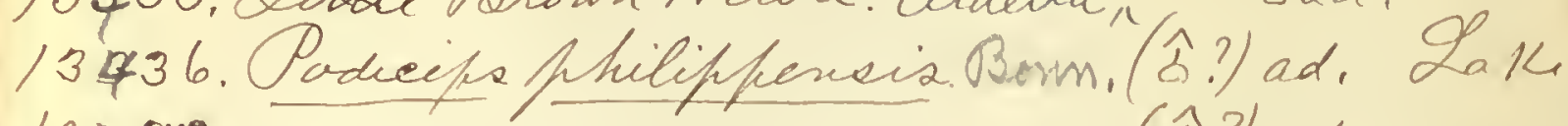
13890.

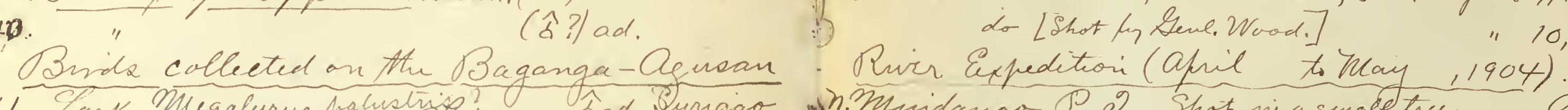
P. 2. Opril 6, 1904. Basayan, bsturan the Yaraca R. and Ragayan. April $8,1904$.

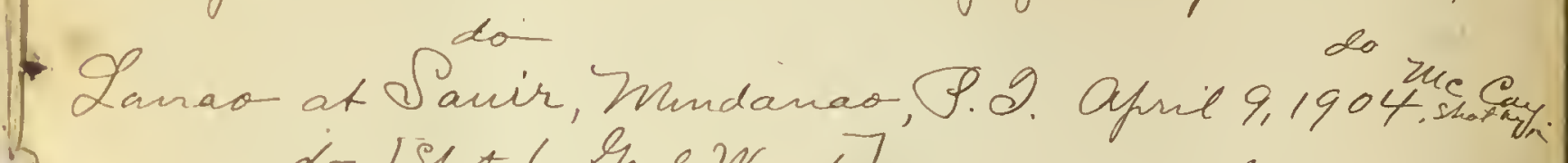

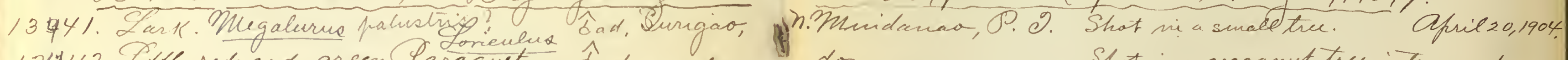

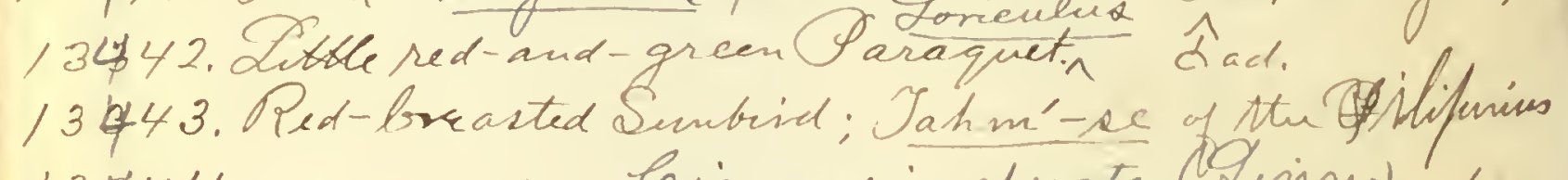
do Shot in a cocoanut tree intown. do

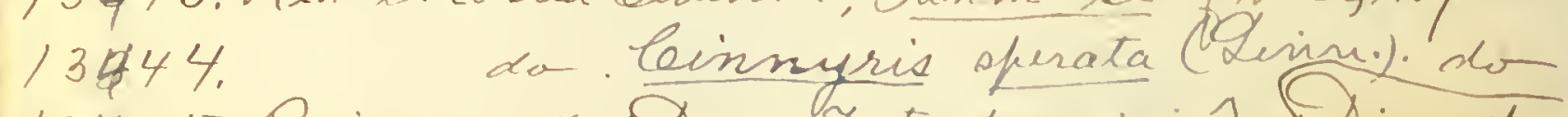

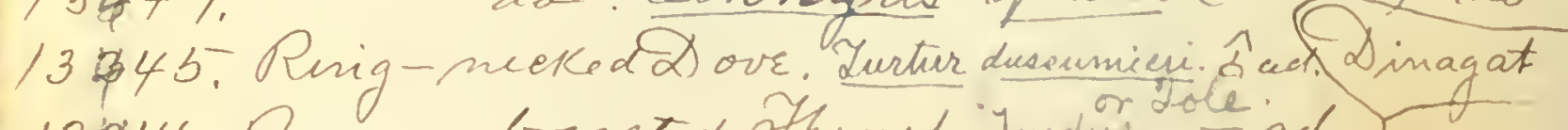

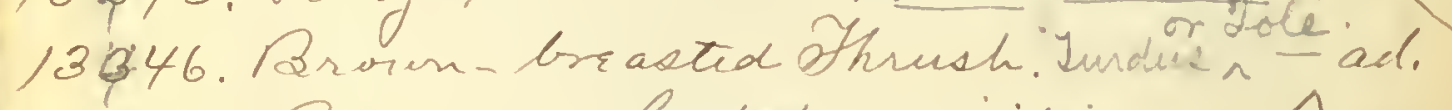
13\%4\%. Rainerow Clentropus viridie. 13448 . Ked-hreasted Barbat,??. D iedeum id. Dimagat 13449. Black- throated a Sunbid loinnyrinc 13 i 50 . 2ask. Mmegalurus paluotri:?

1305, Anall Conslew Numenia

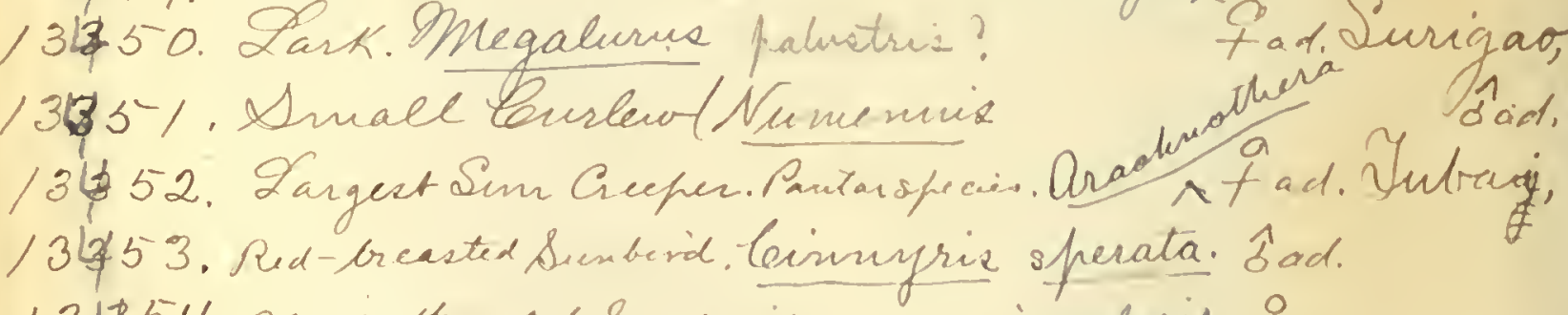

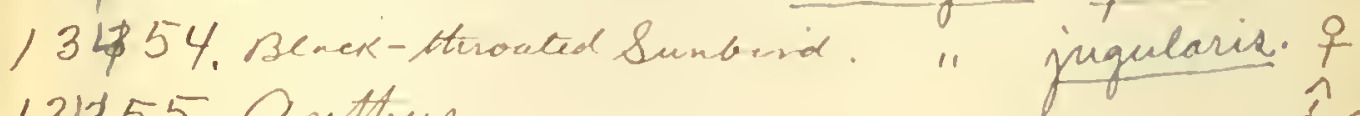$$
\text { t"Boholanos". Bacuag, Ininidanas, P. 2. Sad. Apul 21,1904. }
$$

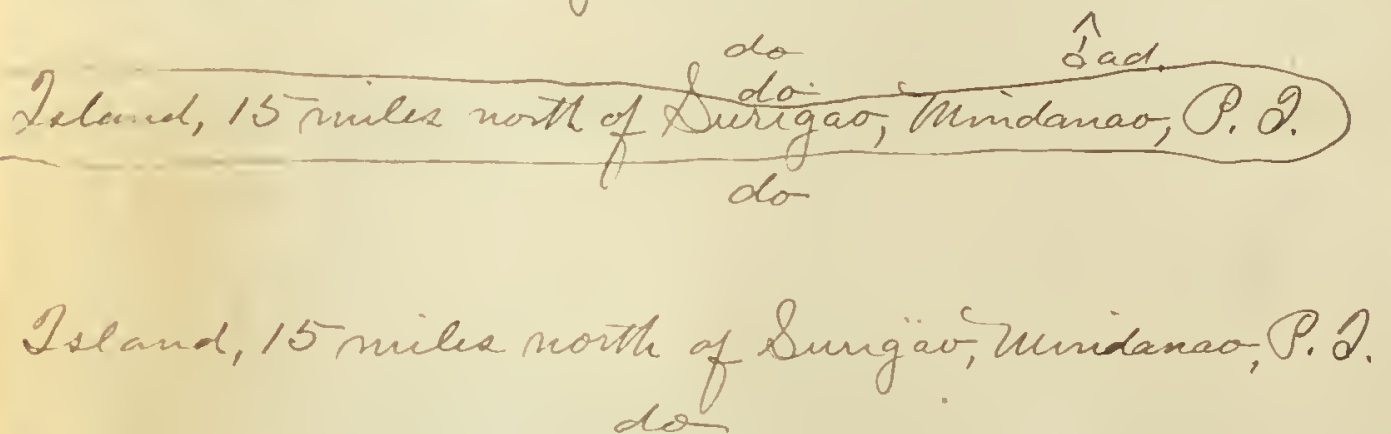

do

do

do

da

131555. Anthus

$\hat{o} a d$.

Tirindancas, P.e. Shotinamall tree. Thatiof tho. 13341.

da

13456. Aypothymix

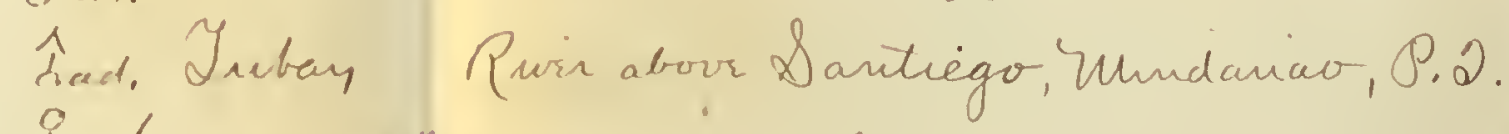

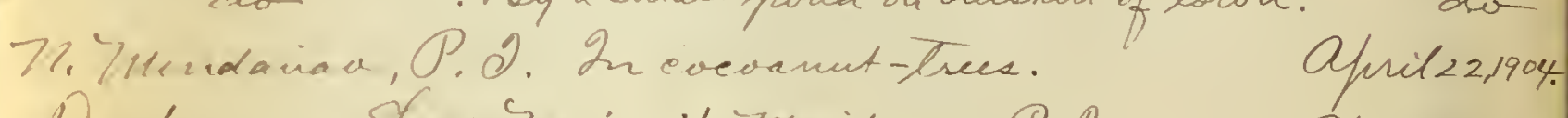

Jabongra, Zak, Mainit, Mnidanao, P. ?)

Afuil 24,1904,

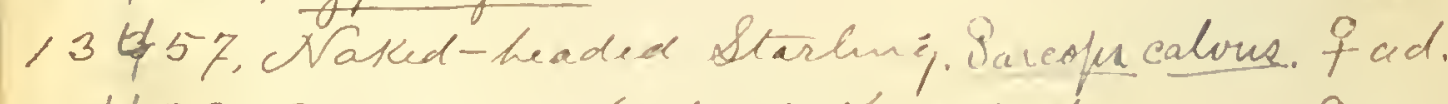

13458 . Beve-and-chestmot kingfistur o ad. Iutrayl

13159. White-hisaded Kingfiaher.

fad

13461. Paquoth-taled Parrot. Prioniturus

13462 . Hyfothymi

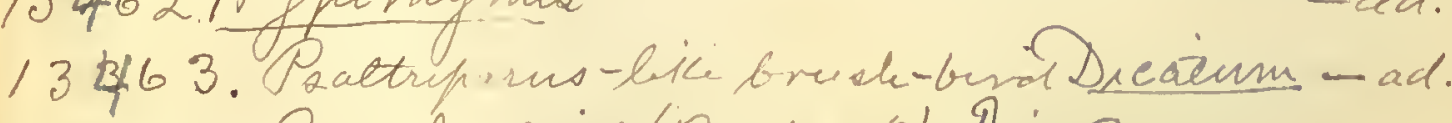

13464. Red-brelied (Barbit(3). Dicaenm

R cla Arforitnig.

do

do

do

do

do

do dom

der

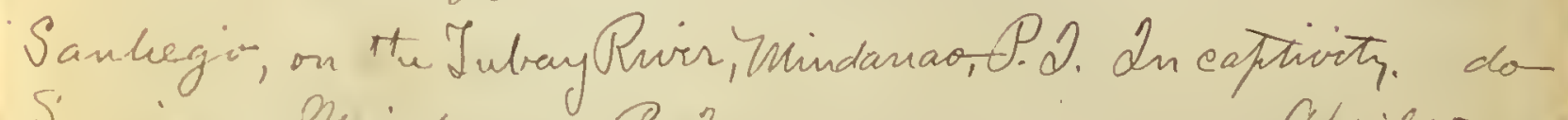
Sinrigor, Mt indanao, P. 2).

April25,1904.

det

da

do 
120

Bolbopittacue

121

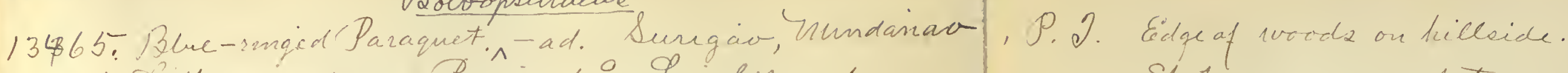

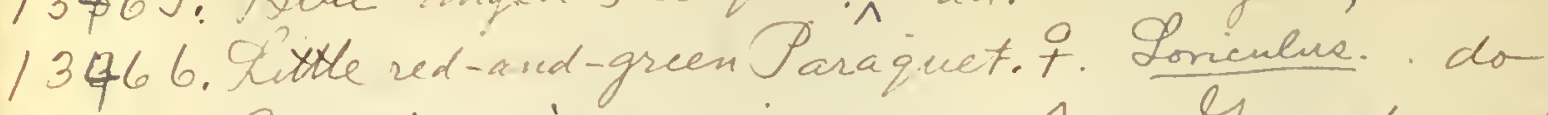

13467. Butrides jaramea Sad. Osandea, repher

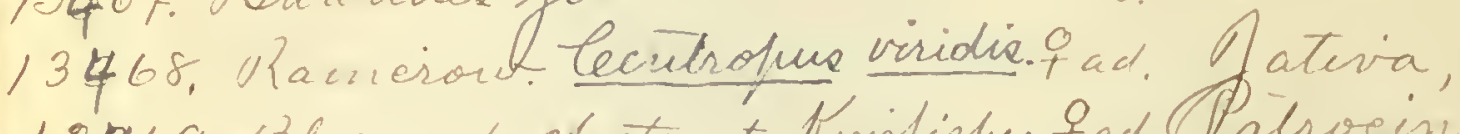

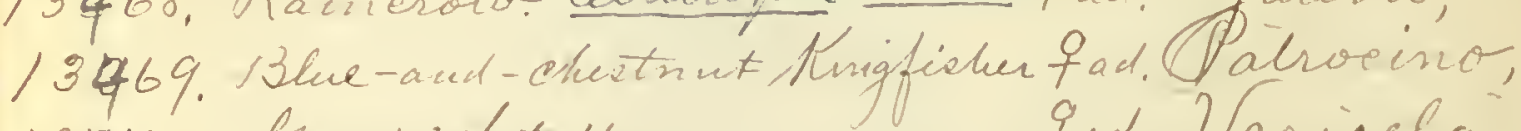

13970 . Suat whete Heron. qad. Varicelai,

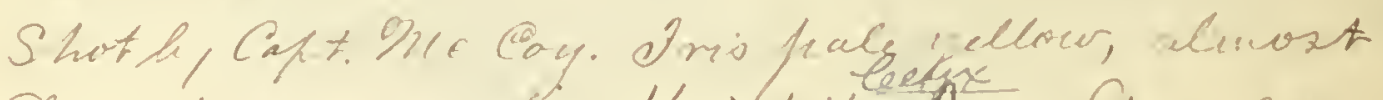

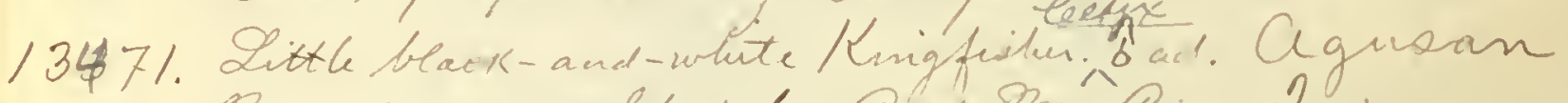

May 9,1904. Shot by Cakt. Mne Ooy. Qhi viry clanobrownish black.

13472. Hiniantopur levereephalux 9 fould $\hat{s}$ ad. Gimin

13473

13474.

13475

$\hat{s}$ ad,
$\hat{s}$ ad.
o ad

Aquan Ruir, N.Mundanao, P. 2).

da

April 25,1904.

da

dow

Mayb,1904,

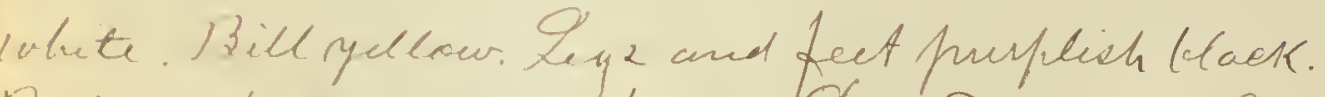

may 5,1904

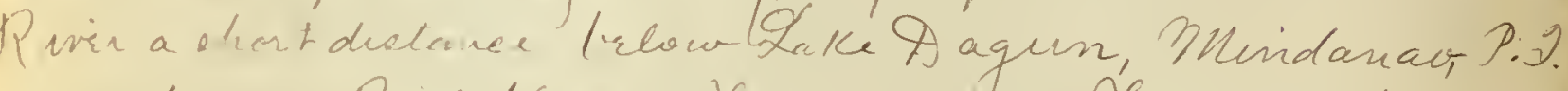
dank trown. Bell black. Yoas, 2-1=3. Fret varmilion;

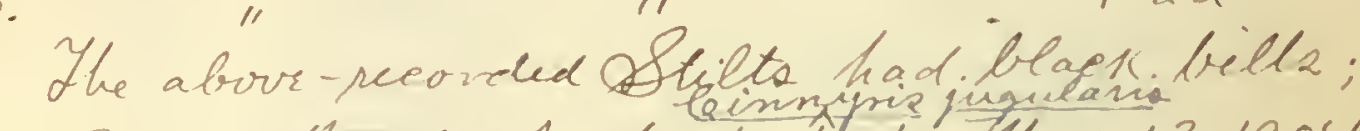

13476. Beach-Dhroatied Sumbird. Adad. Hoy 13,1904.

13197. Anthus

13478 . Bhe 2uaie.

limeata sad.

3, 'M. Minidanao, P. 2. May 12,1904. Murce fielde.

do

do

do

13479

13480 . Thang:ove 2varbeer.

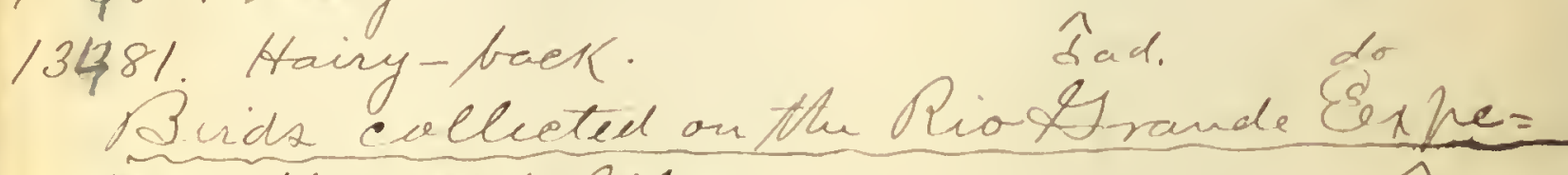

13482 . Hydrochelidon

13483.

$\hat{s} a d$.

do

do

red iriv; purplish-red le gs and foet, with duekr clava. Larigaron, Mi Mnidarav, $P_{2}$. S. Shot in a cocvanut-tree.

do

Baliangao, M. Mundanao, P. I. Shot ni a eonotifield.

der

do

- diterir (Vkay 14 te , 1904).

2rie boun. Bill dark red. Feet dask sed, cleurs

13 \$ 84 . Little qray Tail.

13 485.

do

iे aी.

The two above Rails wre colernd: Baic yiconer,

138186. La-wr'-ong (moro). Hyfrotanidia tral.

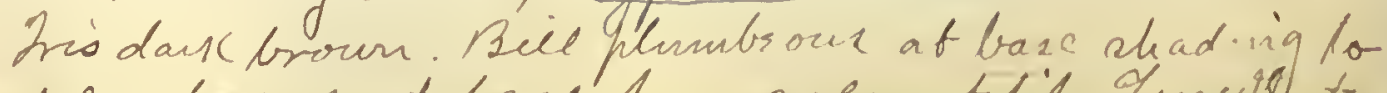
plumbrous at base, hom solor at lif, Lencith to

do 278;712:242;8:2; culn, $33 \mathrm{~mm}$. Thay 18,1894.

do

det 220;320;97;47 mum.

dor

al aceous aberr. Iris red. Feet and claus alcir. do Zuke Bulwan, iundanao, P. I.

Thay 22, 190\%

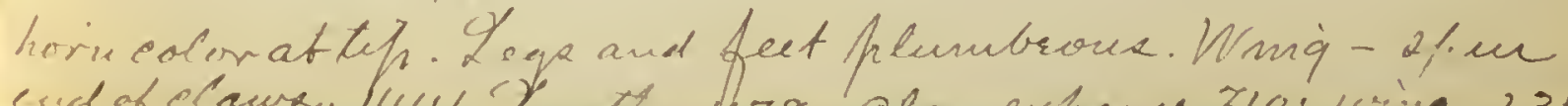
end of claure, 444.20upth, 478; alar expance, 710: uning, 235; 


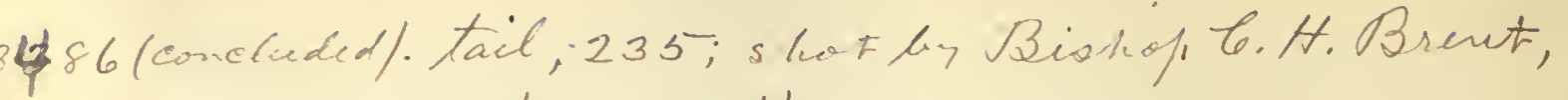
22, 1904 (2rhitiounday!)

3487. Pa-han-ook' (7icoro). Hypstoremalia? fad. P. I. Pnay 27,1904. Shot with buckelest without Frovial ahield, with crestheel, vermillion, quindy tase; teinimal half black, but of anite, horn jinte; claws duckypliv, lmed brlow with quantity of grave, small red eceds, and inscct it carlies its spurs stiexiniy vile cally, upuard.

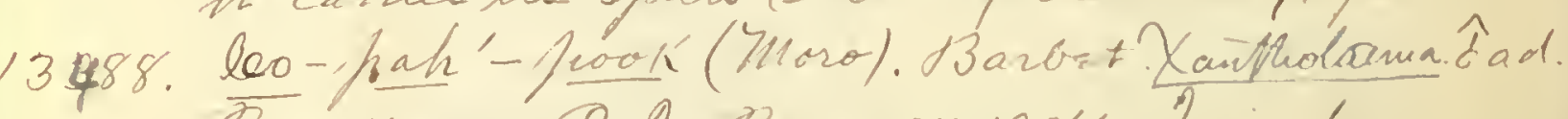
Bundanao, P. Q. Dhay 27, 1904. Ani brown. opace around eye dech wive color. Ficet red buid was sir a small, deceduous thee, utum

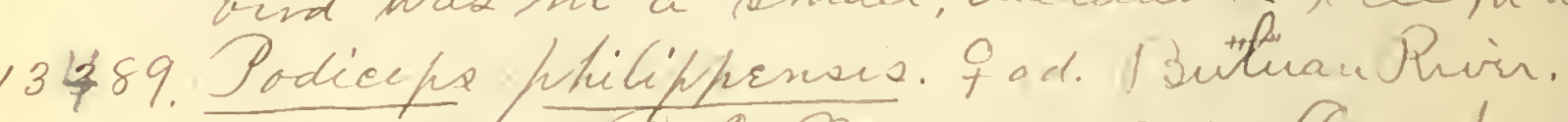

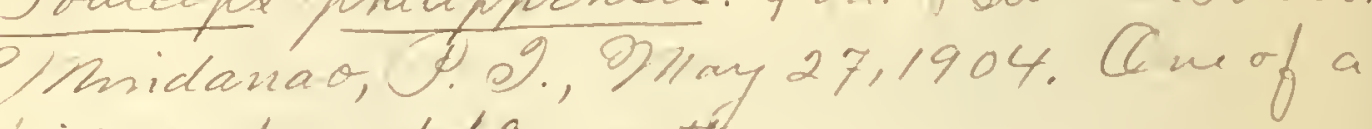
filled with vegelable miatter.

13490. Beaek stäsiniy. Coalormir hanayensis. Ifiw.

13735 Prange - breasted Ereen (tragion. Insthic

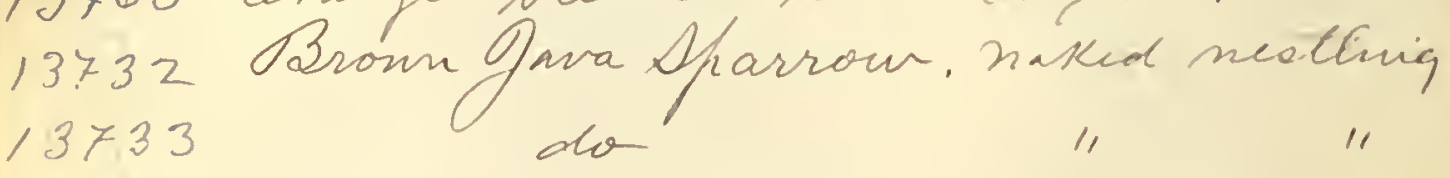
13734 do Nos. 13736 to 13762 antered in covocenhis exlatogue of Boston, inass., on Lake Bucuan, Imindanao, D. 2., May Buluan Purr, Mrturem lakes Buluan and Lequasan, Mnndarrao, sporknis spenien!. 2ris wood brown. Bare eyclid hate beue. changning, after death, to dule, purficich yellow. Sisll white at culor at extreme tip. Legp and futdule gray, gremsit at homevlor. Hezjand nearly empth; cosila mid a small remanis. Trote a low whistled hemulo. O urnig fleght Atoundant on Take Buluan, I. Regrasan and Butran Piver. Pahn'-dog, Butuan Pivir, Geturan lakes Buluan and Suguason, Bill blark, horn ealar at ex lrene base of mindible. Bare Claws hlumbrous-black. 177; 288; 86:45 mm. This to actrons were like those of an ordmain bird.

below Pasdory, beturan Eake Buluan and Legivasan, flock. This femailewould ven aoon have lard egga. Hzzand Zambronga, Mhriana, P. Y. May 6, 1904. From Corlonel torbusier. Mercedes, Mindanao, P. \&. Dece,20,1903. In formatin

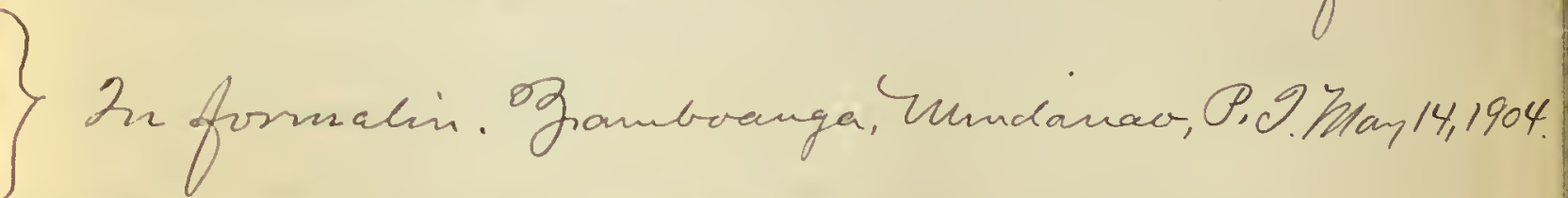

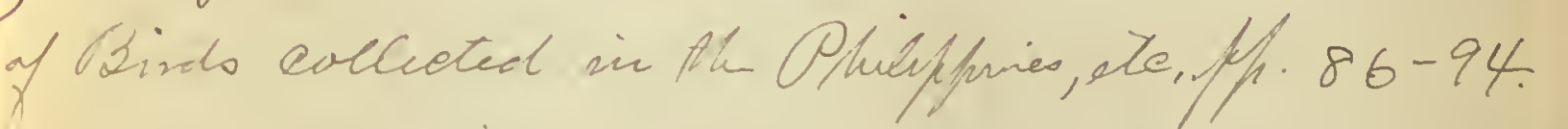


12.1 Birds obsenrd from Ma nila to Seft.19,1904:- Nagasani Day Serns of tho sfecies wore seen. The larger one was probabery Dterna brigie, the otter about the sprearance of

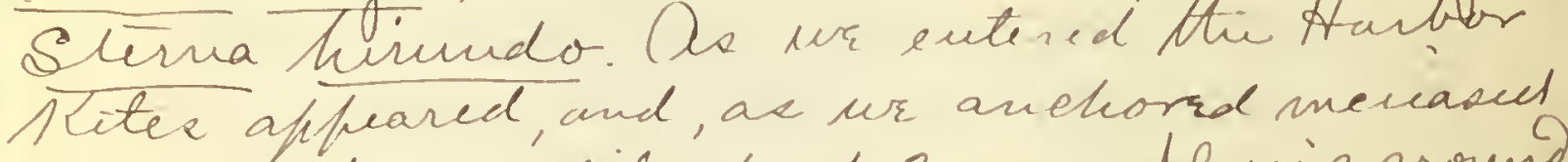

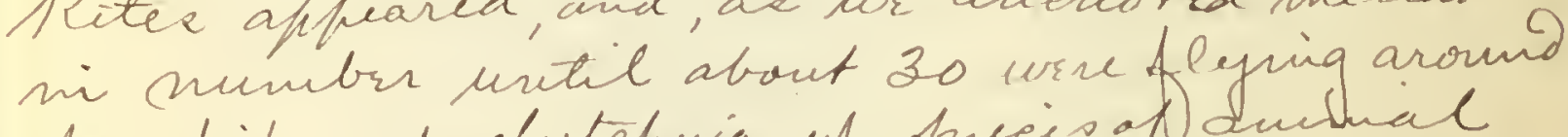

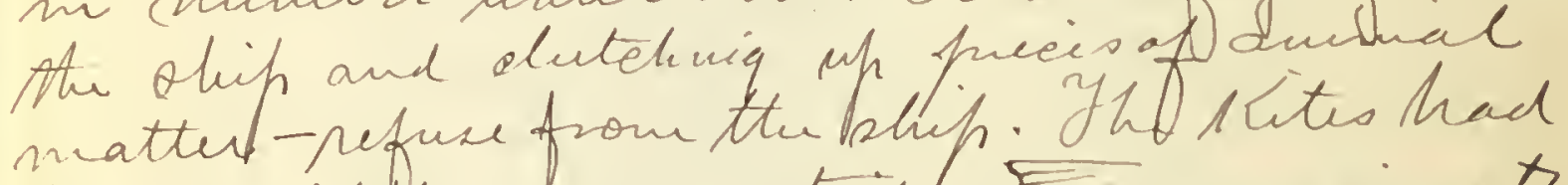

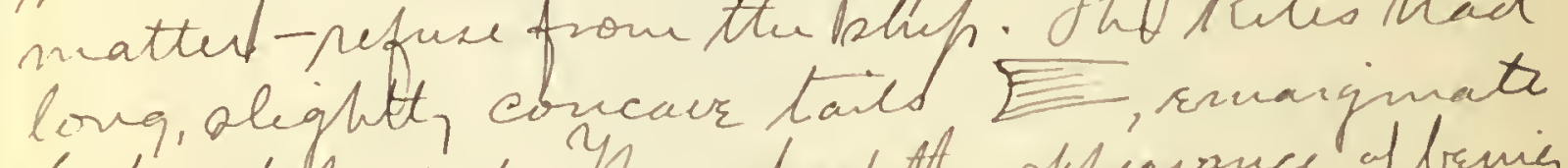
but not forked. Ther had the apfinsuce of bermis spotted abovr, longiturinally stifieilbrlow, dark theoth surfaces. Sone wre nolderig quills,

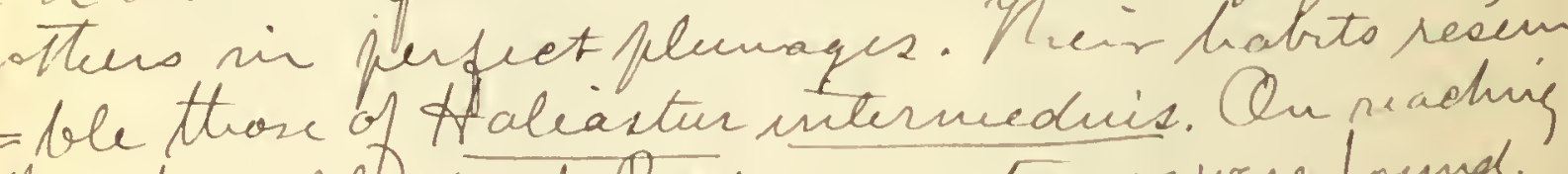

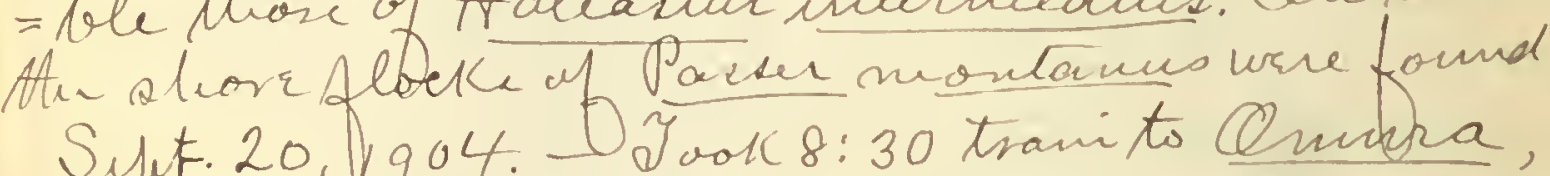
S.p.t.20,11904. Jook 8:30 tranits Qmera,

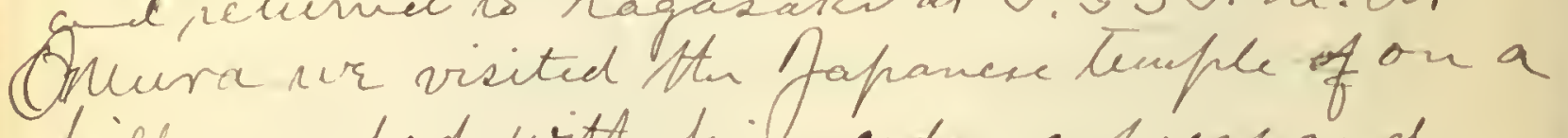
hill uroveded with firl, cedar, cyperse and

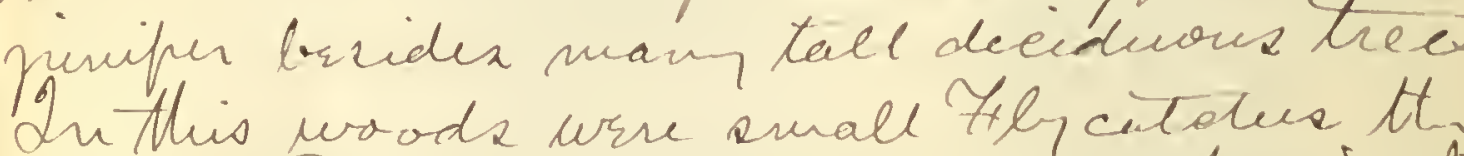
aize. of Cenfidonax; yllowish bids kavring Ithe

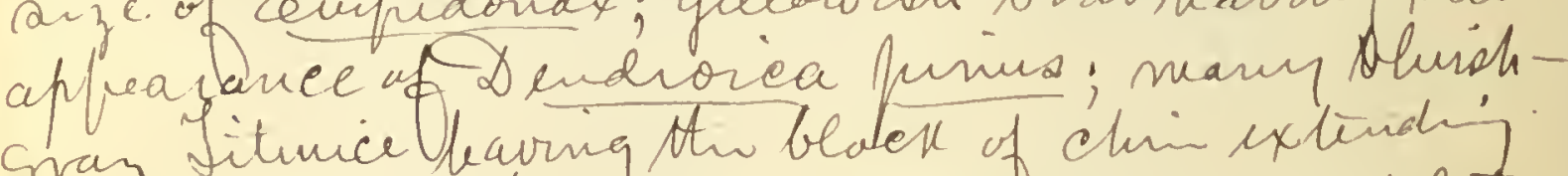

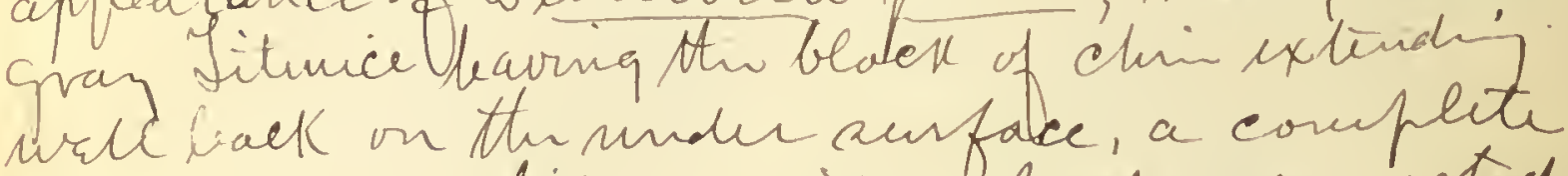
bluer collar, white on ride of head, not arested. These wire nimerons, tamie, a e canne clase ux when clusped to. A foird ahofed like Andyter herd a black strife on Nie under sich exkendung beers and corrring the whole1 25 maderside of the tail; lager broum birds wre Tourne nitennediate in reze v vorieb beneran a large while - branted L t auk flew overhead, Suallows a de Wagtails wrefrequenter

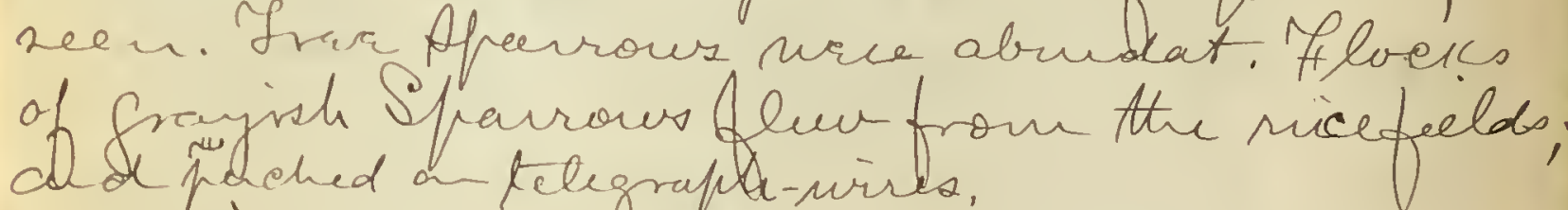

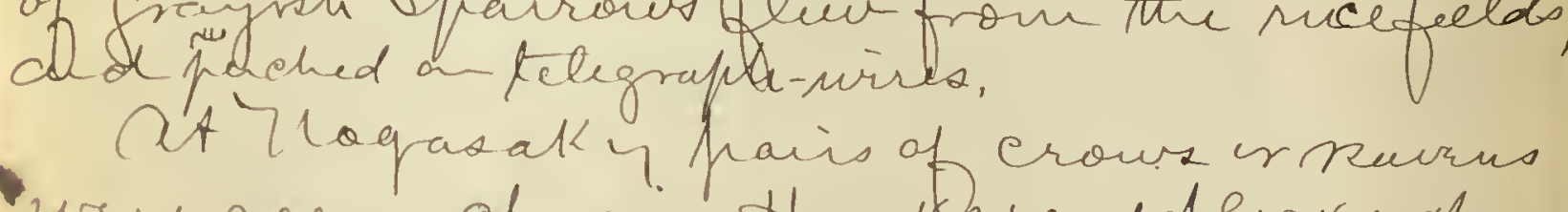

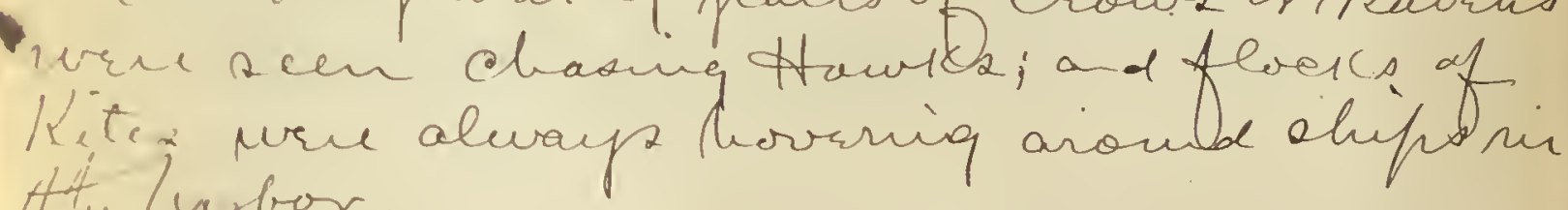

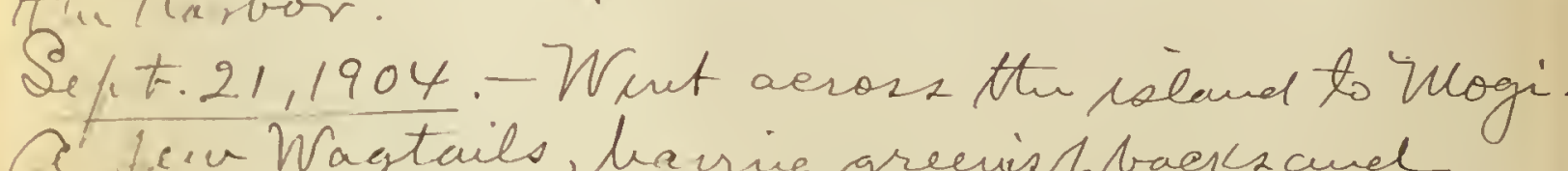
Wagtails, harring greevishtoencsanel Felcon- brasels frequented soekn bede of streanes.

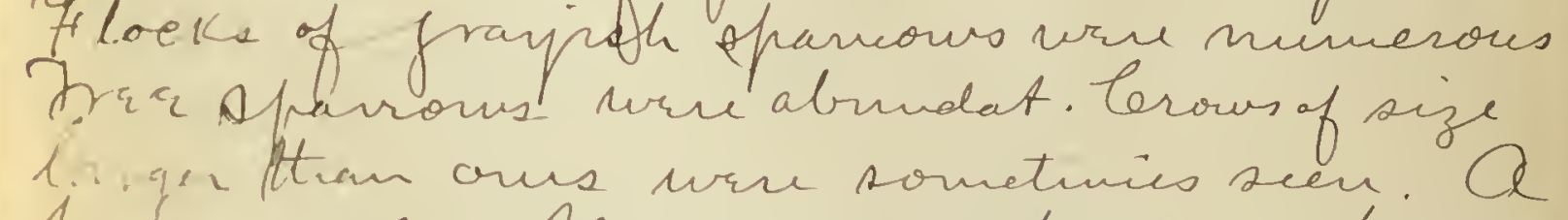

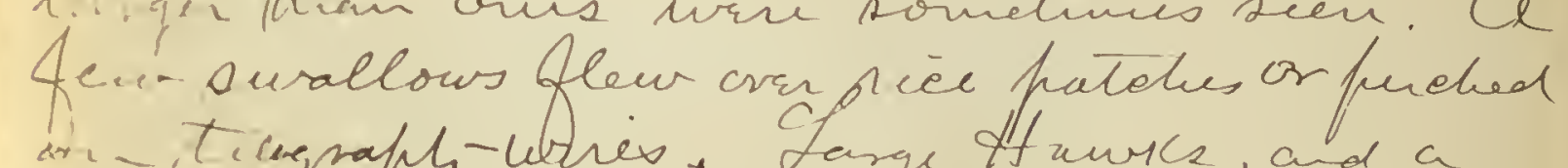

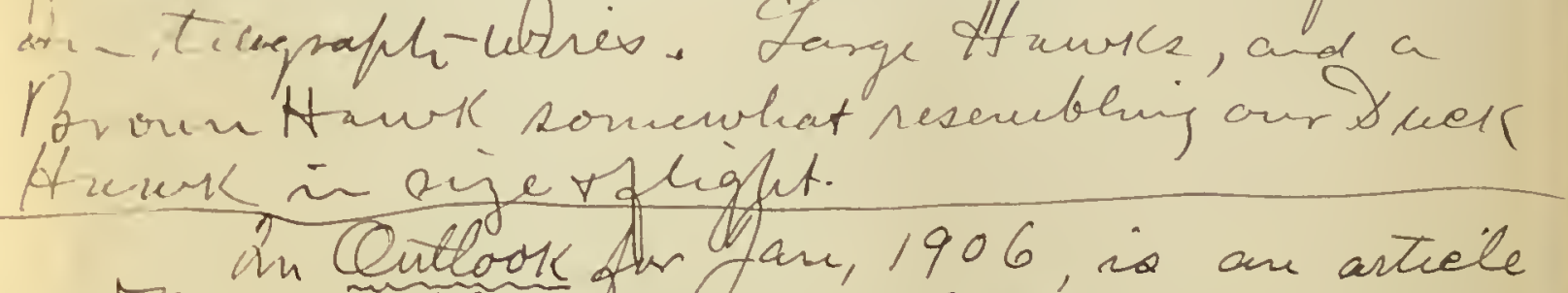
on Thoro fioblem, leontaring frictiun of Ataboad,

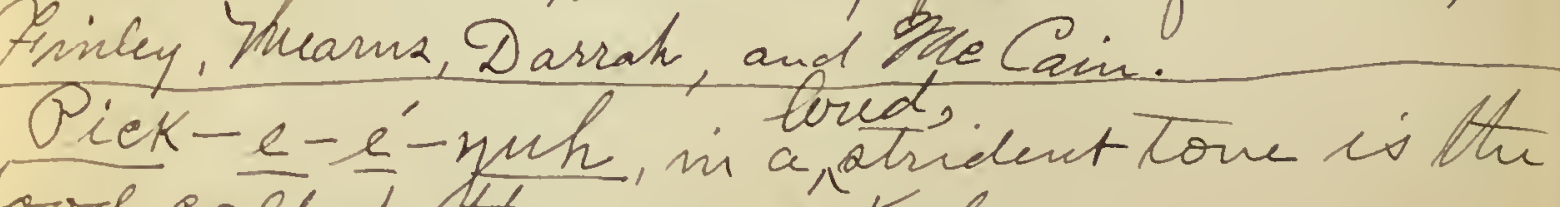

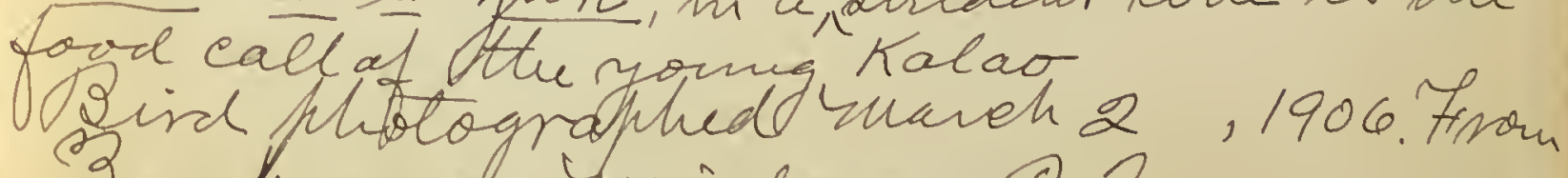
Zamboanga, IUindanao, P.C. 
126 San Ramon Farm.

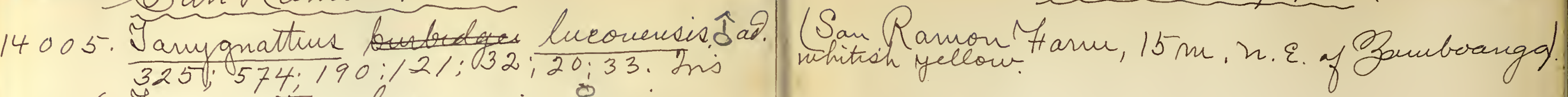
14006 Tanygnatitus luconensis. \$f juir. 14008 Aaleyon chloris. $A^{7}$ ad, Contaive a full-pized egg. 14009 Faleyon chloris. 1 ad Unated with th abodr 14010 Artamide Kochi $\hat{s}$ ad. Ore of a hra ir sen togettier. Dris whitish ycllow. Bell, bet and claws tor $290 ; 490 ; 158 ; 119 ; 29 ; 28,27.6$

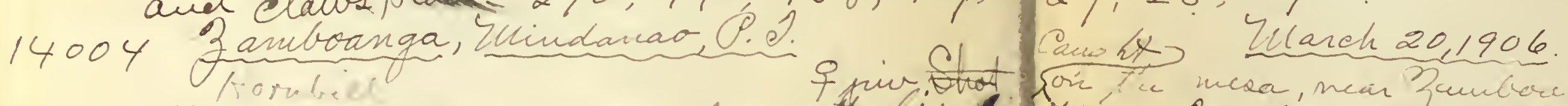

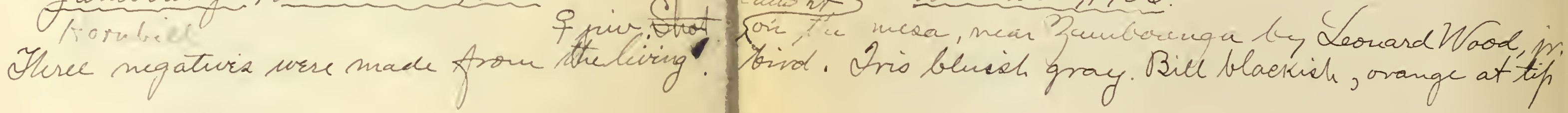




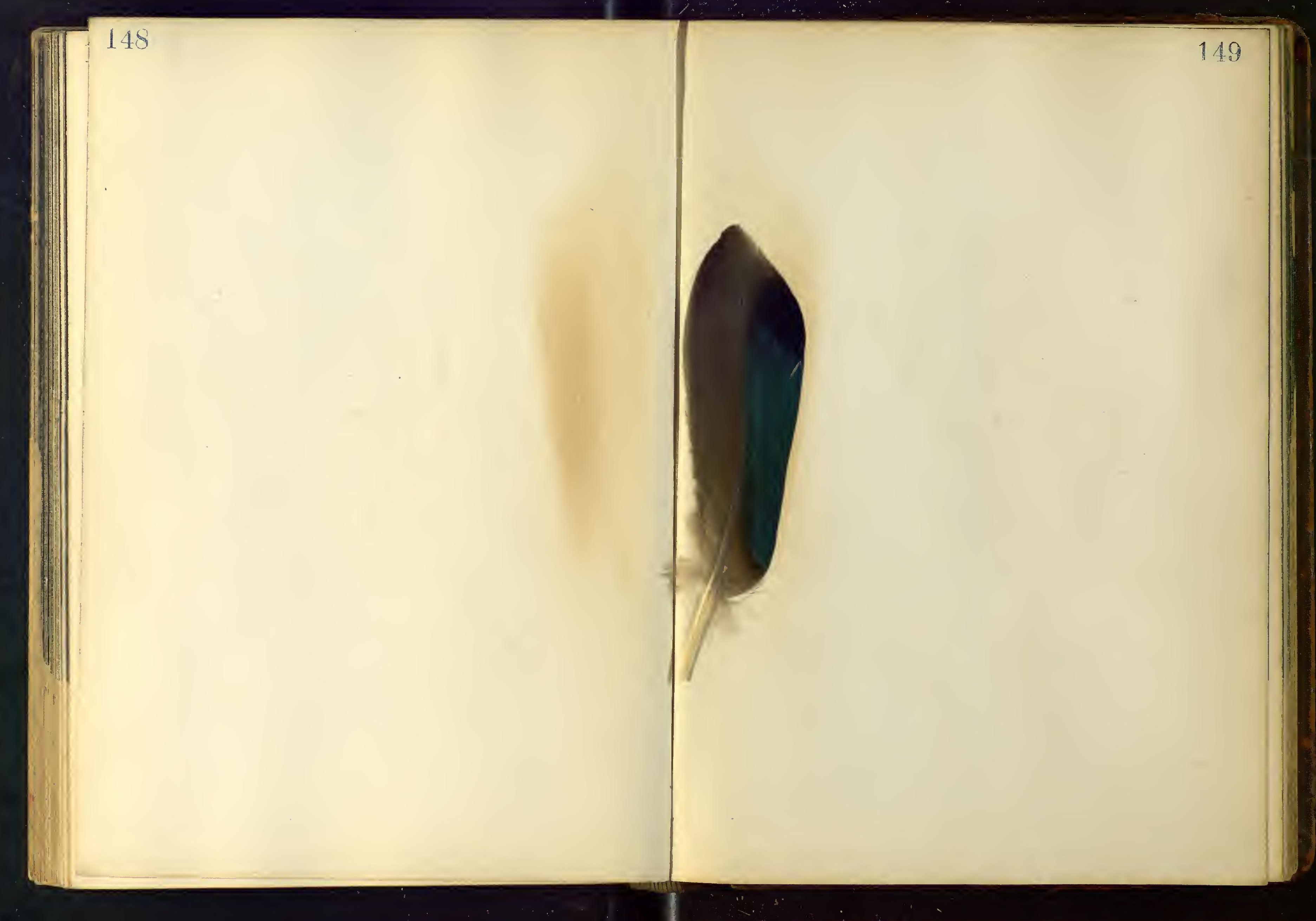




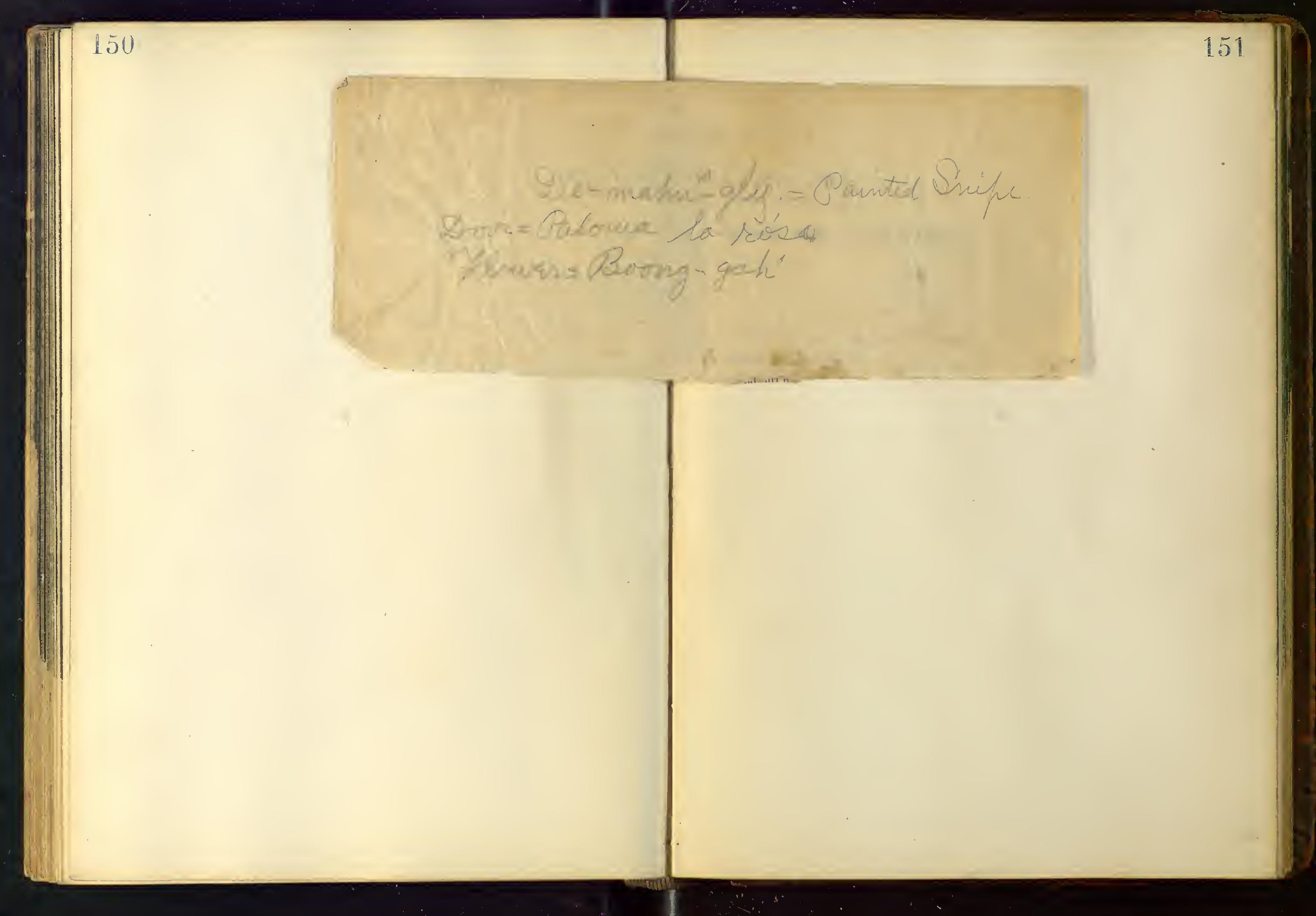




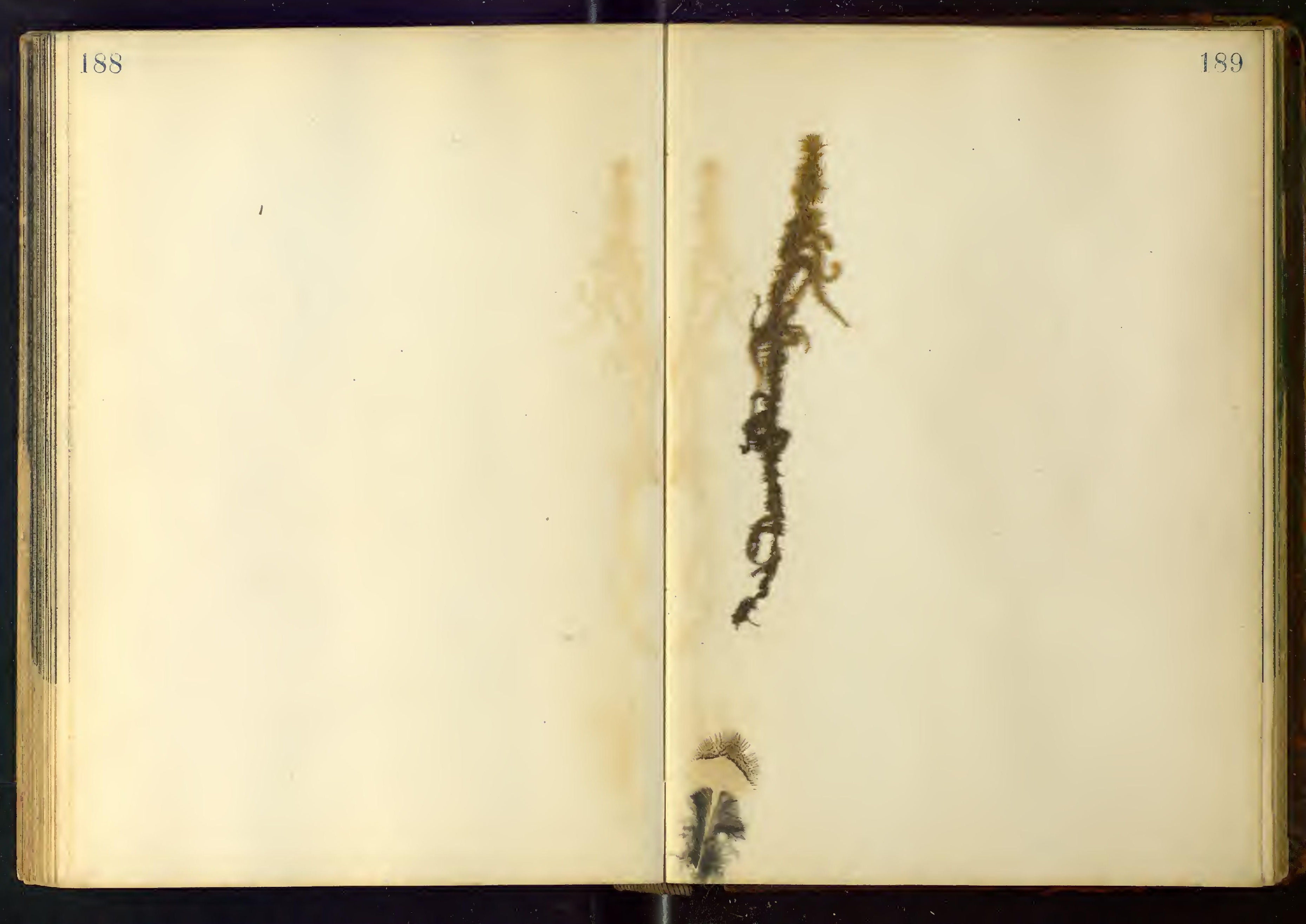




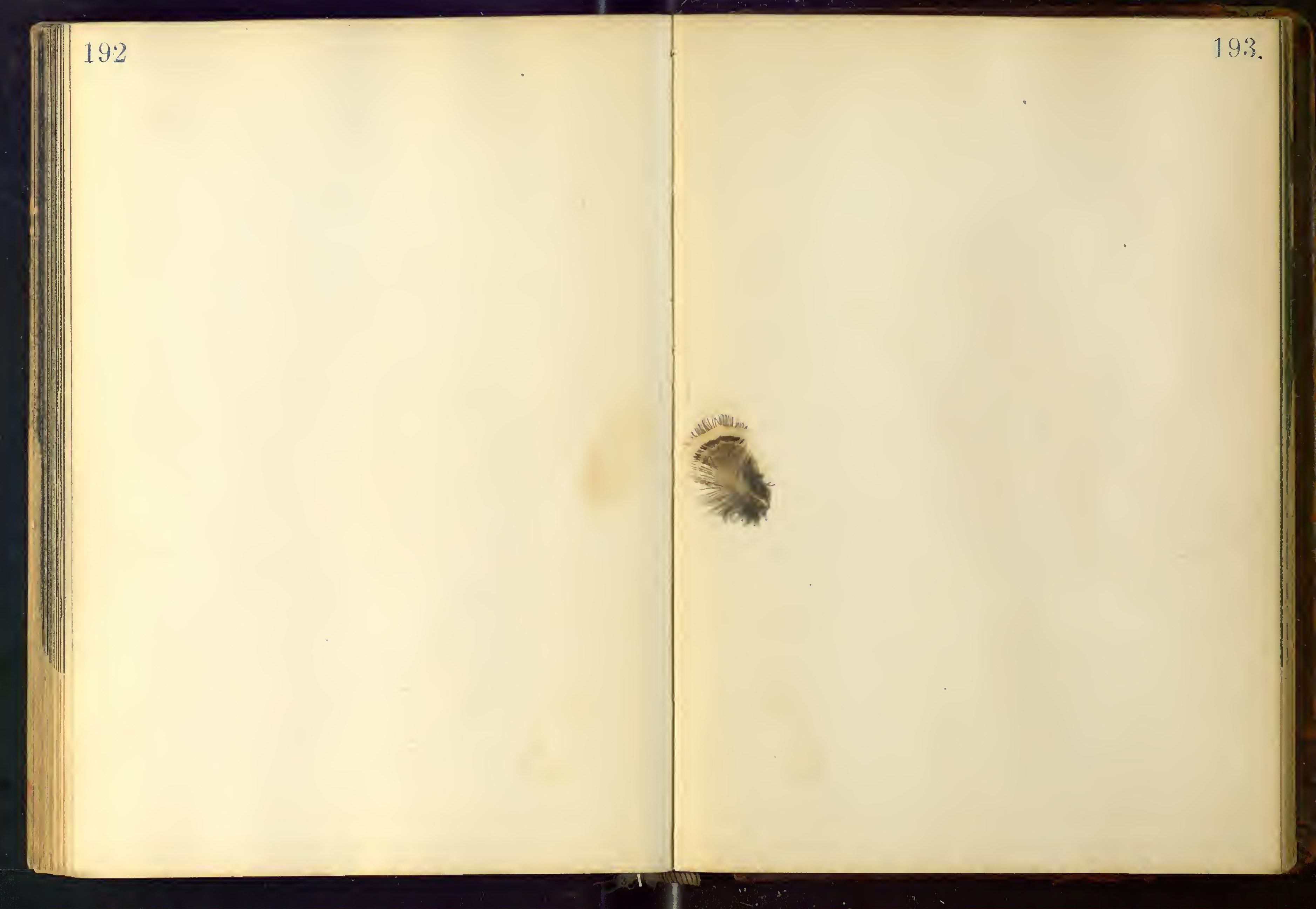


Shit of opreinens sent to the U.S. 2111 No. O

National. Musum in fluida (aloohol,

\{9.9. Long-tongred Swall Bat. In aleohol. Bad. Shot ou under tag side of a everamit-tree, on lagayan Bulu 2sland, P. 2., Fifonam 25,1904.

90. Larger lerab. loe, do fteb.26,1904

989. Sumaler ". do

988. Small Etecho Lezairal. "ha "

$98 \%$.

dow

1906.

975. Cotabato Inosto's Brain. "Weight 40 $\frac{1}{4}$ oncer" A leorstabulary soldier. Trank. - Private. Died at Military Hospital, Zamboarga, Mindanav, P. Q. Ectobre 28,1904, at 1:30 Am. Brain revoved 3 P.M. same aldail. Age - young man. Wright of brain.-Seelabil) G.R. Harris.

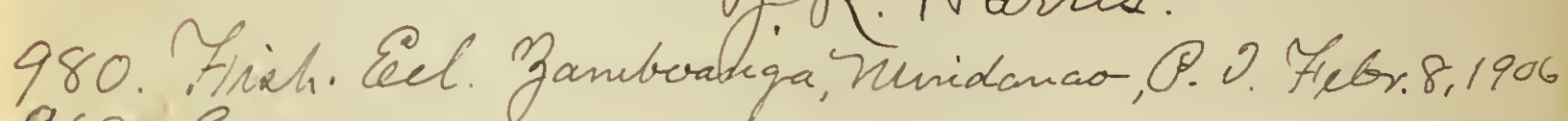
968 terak

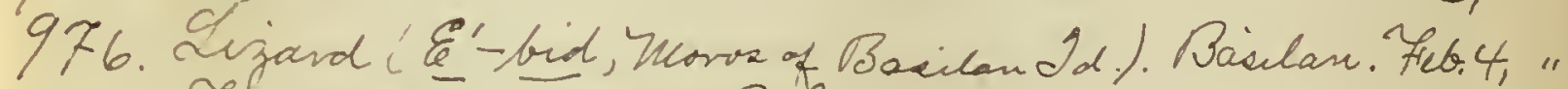
974. Firh. Shash-nosed Eel.

Iainboanga, huid., P. O. fare. 21,1906 Covlucted by Eapt. Frank Ros a'the Coy, nea.

667. Dug (in glan tubr). Zamboanga, Mnidanav, 0.9. Febr.5,1906

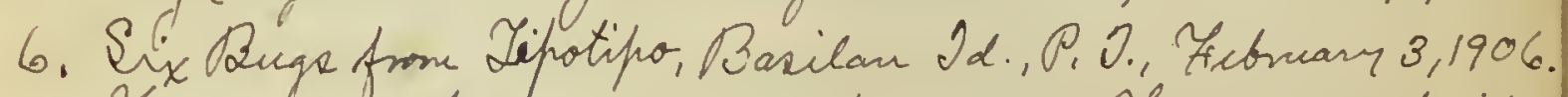
Iransfured from alcohal to formalin. From ald teribe

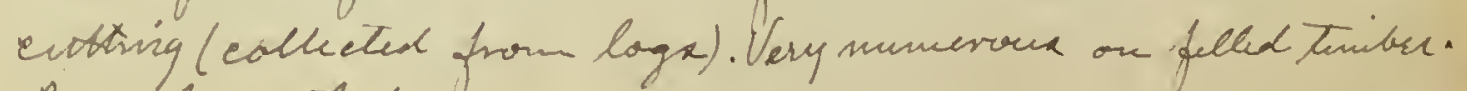
In glase tubr. 
202 (Called Calyaluhe by Manobo nativis),

97\%. Lizard strm Baht 2d:s Sarangani groufs, ofts. A Inidartao, P. 2., Gan.23,1906: An abrinlants specie in the monitame. Luv (exacth alie) were shot from trees; ales seen on the ground. Wpper fraits a ateeriorly and oll of miderfiaste green, which became blue in fonualis

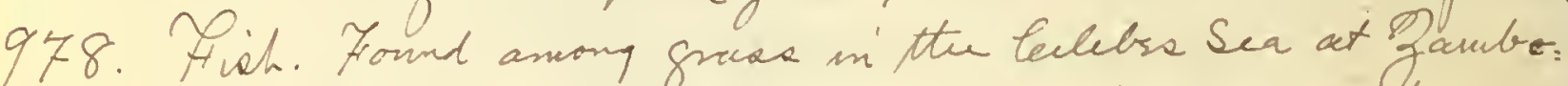
: anga, Suindanav, P. 2., Fictor.8,1906. Cearght on the Priface, while 2 war in bathmi ni thi surf. fornd dry in a susted-out can, at the ONilitary Arspital, Garuboanga, Hundanao, P. O, in Gancary, 1906. Dt had Ahobably bren inserved in an acid salietion of fonnalin which had eaten through the con. Dr. Jeses which Harria, US. Ami can perhafer Aurnish dater, as it was with ofreniens furoesved ley him

979. Long, mellourich Dea Serpent. Platurns? Aaw.8,1906. Jotal length, $1970 \mathrm{~mm}$. Tail flat, 40 mbl. wide. Ereatest defth of body, 60; cincumference of head, 90 ; slenderest hast of neck, 70 ; greatest arrimuference of body, 120. Probably no food in it. Undercide Datraul color, with a suggestion of the dusky banks of upperparts. Postinion half eonfreased, neeled above and below. Ercatest deptte at a point 360 mm. anterior to anus (60 mm.). 46 Oliv-phubrous dianond-shaped areas from haed to ind of tail, 969. Short, thiekset, of ark-colored Marine Silake. Jotal length, $1265 \mathrm{~mm}$. Tail 30 mun in width.
969) treatest depth of body, 55; circunfernec 203 head, 90 ; circunference of slendercat prast of neex, 70 ; greatiat circimference of body, 150;46 alvi-brown of yellowist brown contined on sides as for doun as the broad yellow (straw-evlored) belly sentes; theece dack bands separated by cinereons bande of ofposite faltern (broadest brlow and narrowate alove instiad of the reverse. Note.- The above snakex hannary with 1 about 5 miles of the Doland of Avere, in ther Iulu Dea, in a school of thundreds ared probably thonsande of individuals, swinming around the phif (U.D. A. Irensfort Dabah). Sevral ivire shot and two taken aboand for freservation. An the Aabah stearued on her course, the snakes wrre in sight for an hour and a distance of eight (8) nicles. The tivo ofecinens wre freserved in formalin in th sunce receftiele. The pualle bue was shedduig its skin and pras popt and mushy, while the Congext ofecineen was preserved without diffiéeth. A large masine snake was seen two days later (Aan. 10,1906); ni the Aulu Dea, Gelevarn Ht Bolod 2do. and Balukbaluk Id 953. Horsefoot Cerab. South 2slands of Sibitu (the most houthem land in Philifficic Lalands) Ganuary 5, 1906.

952. Cecet from Simaluc 2d. offW. coast of Vavi Iavi Dd., P. A., Jamary 8, 996 . 
204

993 . Noid

205

961. Ebid Eadrellus ot Damboanga, Shril 27, 1906

982. Hrad of a sea, turtle. Collectid bi oैor. Harris at ganpivanga.

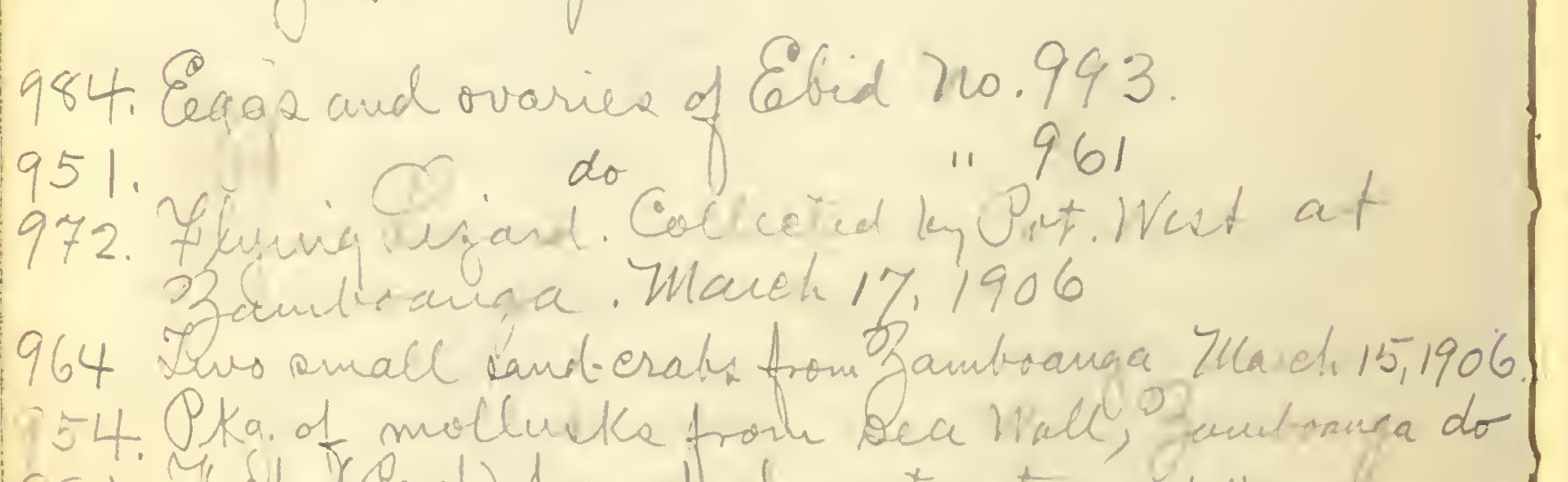

981. Fish (Pereh) frou fleshe-woter etrean at Herry

967. Jaken with rod, 3 sinkes above manth of the ruvr.

the Mlastation of Harry Zuneser, she of of

956 Sasilan, marca 20,1906

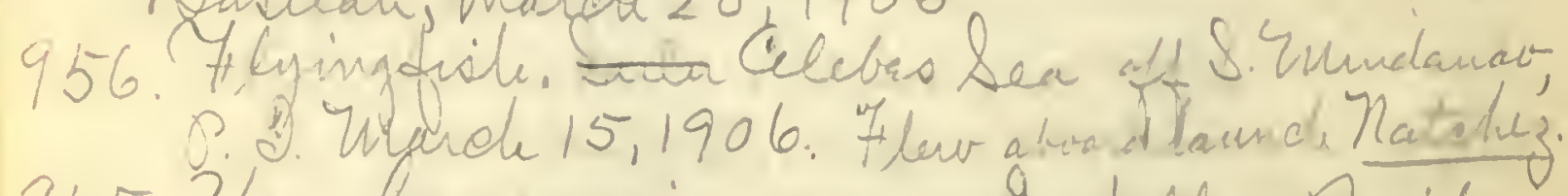

955. Very large marine worm. Isabilla, Bacilan 966 (subsitute ho, forNo.6037) Su ho.6037, f. 30.

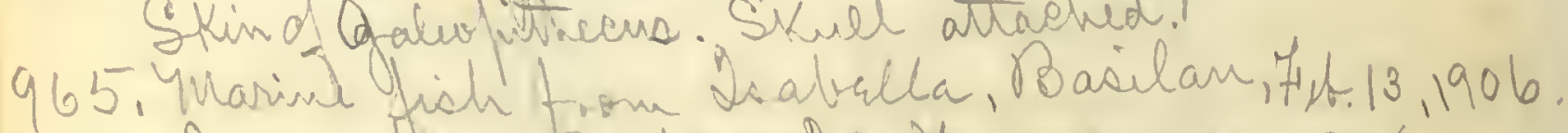

957, Duake from Rasilam 2d, Fisr. 13to22,1906
948.

1 ists

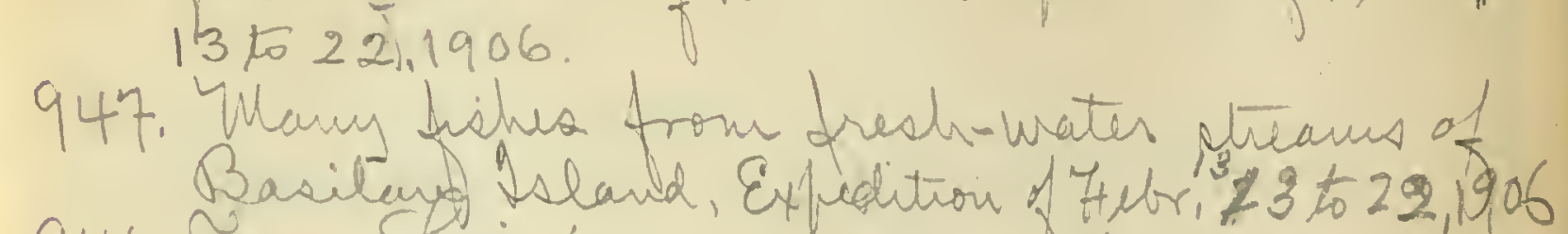

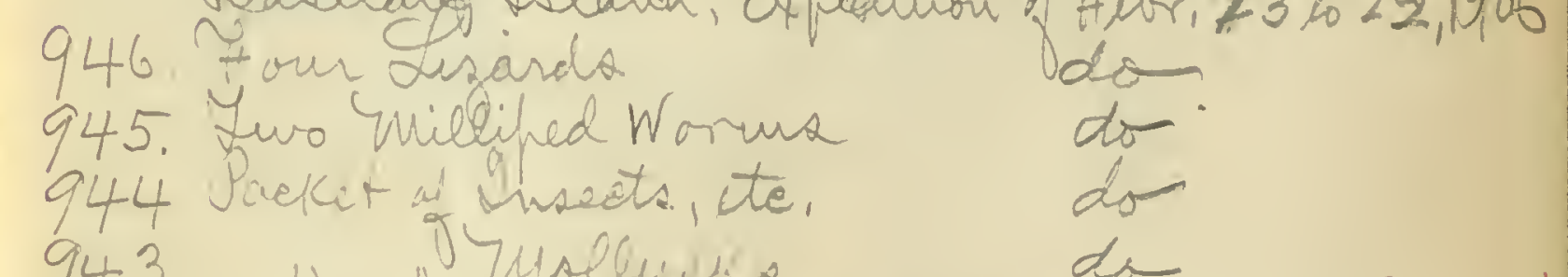

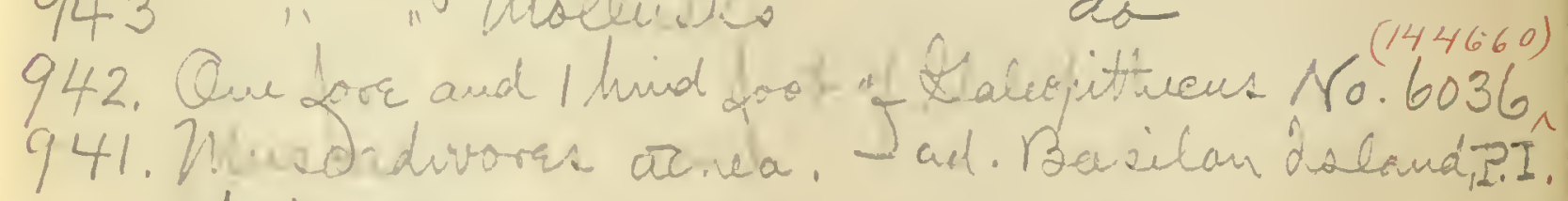

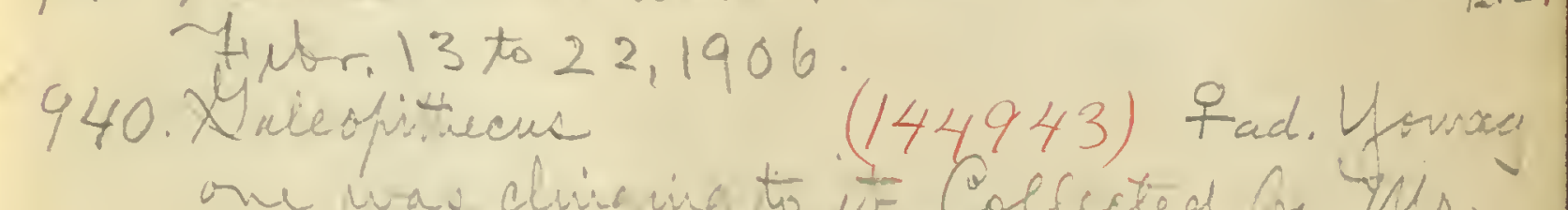

939 wrst dswalla, Do Pacilan Fitr. 28,1906

936. Unitte. J naw this in a dech wrill (made Cy

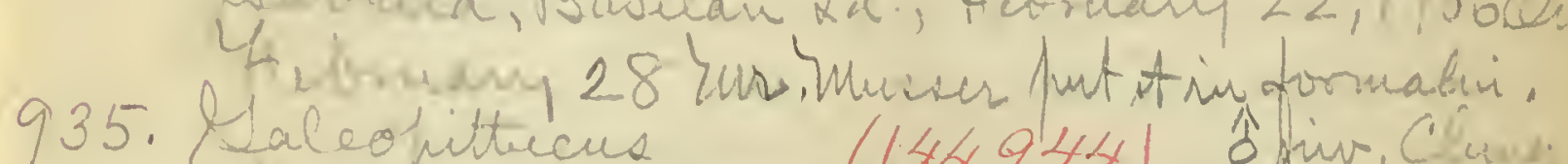

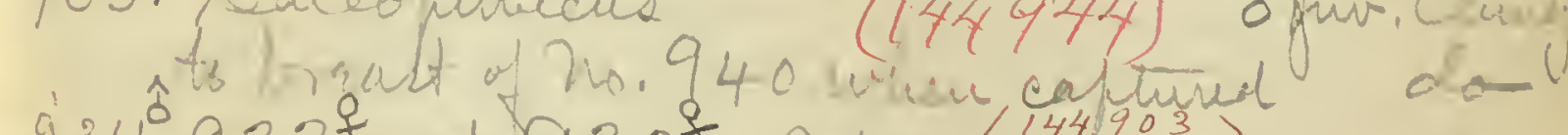

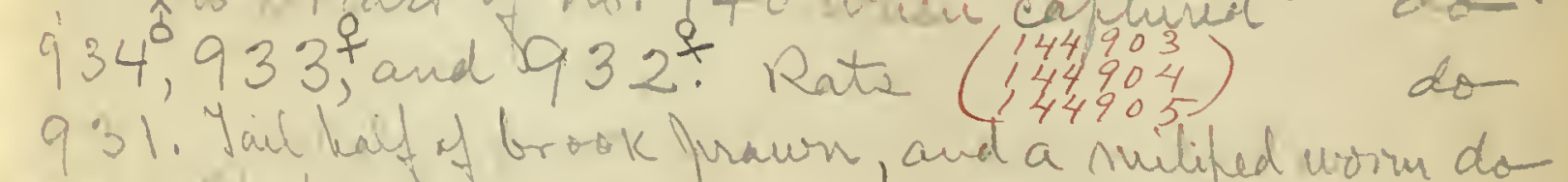

$930.2 a t$. (144902) Fad. Tlluivers,

929 Sasilan. Feh 20,1906 .

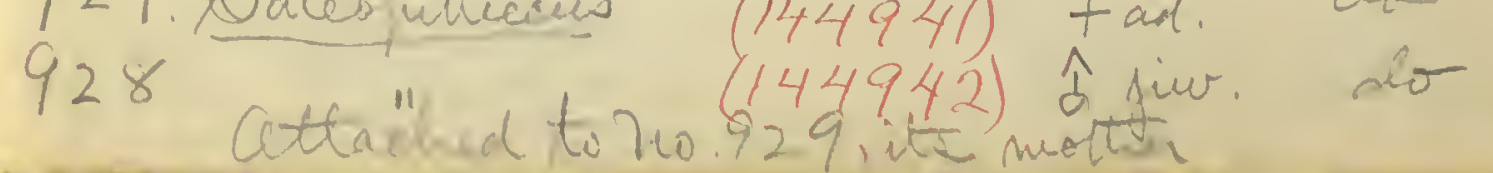


206

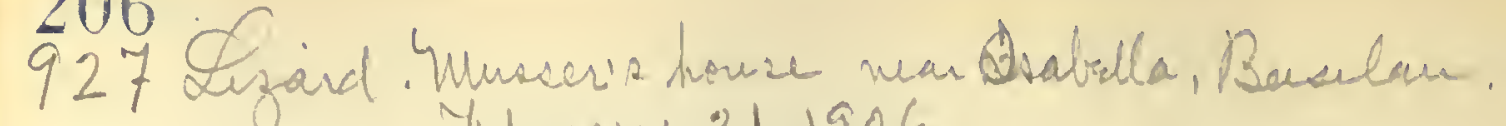
Fitruary 21,1906

926. Tuo frosh-water fishes. do

924. Proongess Snake. From Willer's ranch, nur Zrabllla, Basilau Leland, P. J. Fefr, 22,1906 Qreserved ky Yhr Teakle.

23. Ltar-shaful Urduir. Ditte Santa Cruz 21., of Oaniboanga. April, 19060

422 Sterra $(1448.82) a$ ad. $=n_{0} .14069$, f.18, Petat.

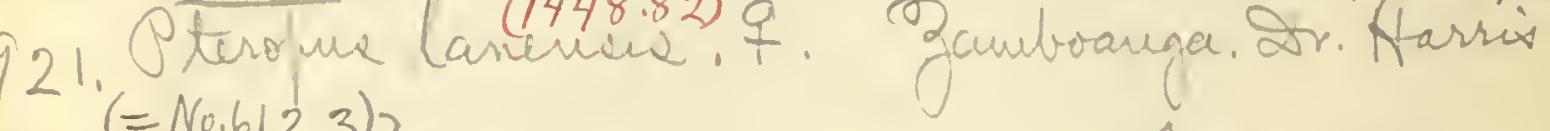
$(=N 0.6123)$ altached to its mother, No.6061. See hase 32 and 36 Narnie liant. Iitte Dantalerus 2d; off gambongas, Unididarao e. S. 1906

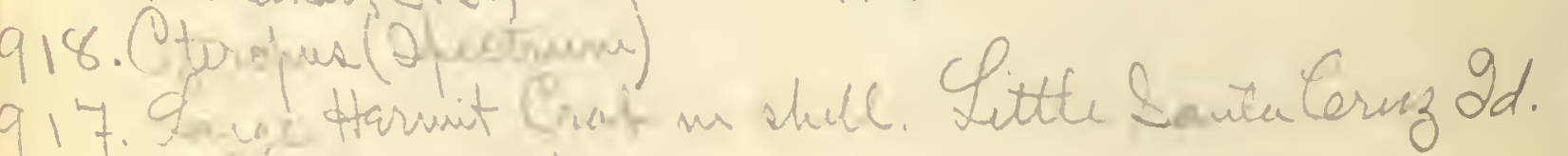
off Sambeave a Thidanao

916. Runde of Starticites

1906 dor

15. lennomolans mund(844939) is ôn. Hzad. La uboanga, Mnidanav, P. Y. Dr. Harris.

914 dung of above

913 sincer $\operatorname{dinm}(2)$ of aberes.

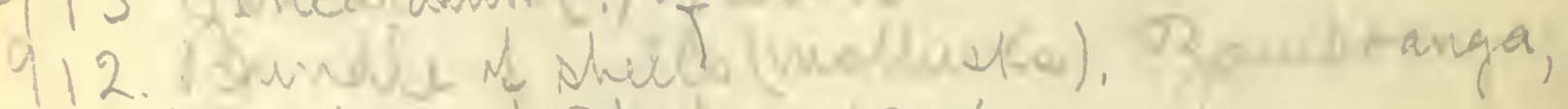
Uuridarat A/uil 4,1906

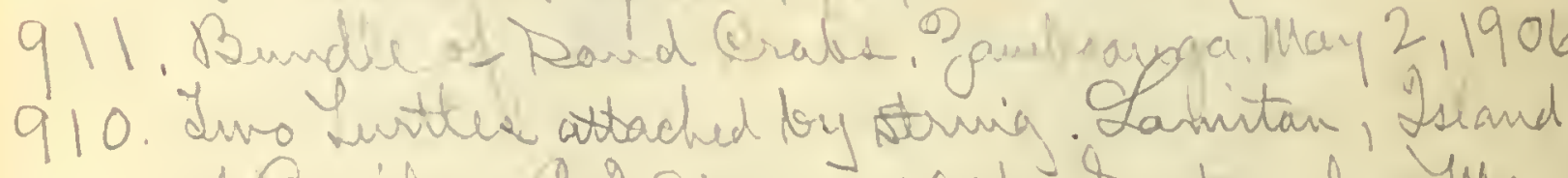
of Bacilar, 9.9. May 2,1906. Sent me ley Th. Bhomen. Welt alier in my rooin for sevaral warke. Ate cooked pier ravanonsly
909,908, and 907 4 it 207

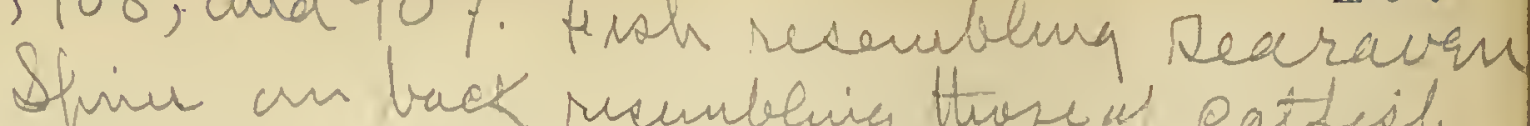
aid una mora forsononk. The noturas as in great dread ite prieks, which make very aurre wonide! Called Los-foh' by

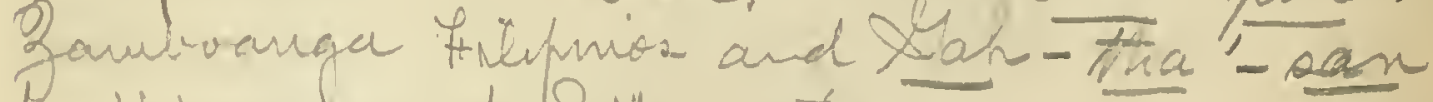
by Visaygans. of Sibu, te. 


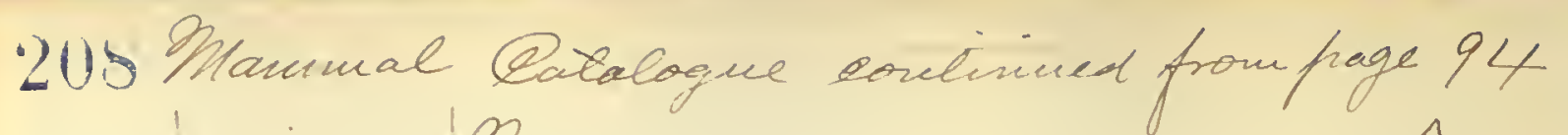
6596143047 Mnus

6597143048

6598143049

6599143050 Ohms

6600143051

6601143052

6602143053

$660314305 \%$

6604143055

$6 \cos 143056$

$660614305 \%$

6607143058

6608143059

6609143060

$66 / 0143061$ $\hat{o}$

f

q

$\hat{\delta}$

$\hat{s}$

$\hat{s}$

$\hat{o}$

sิ

बิ

$\hat{s}$

के

$\hat{\delta}$

กิ

今.

今

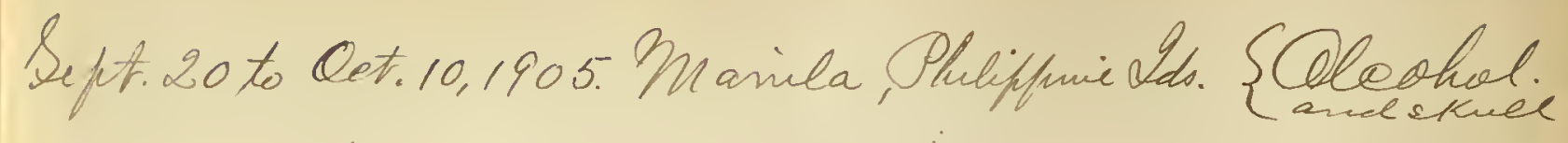




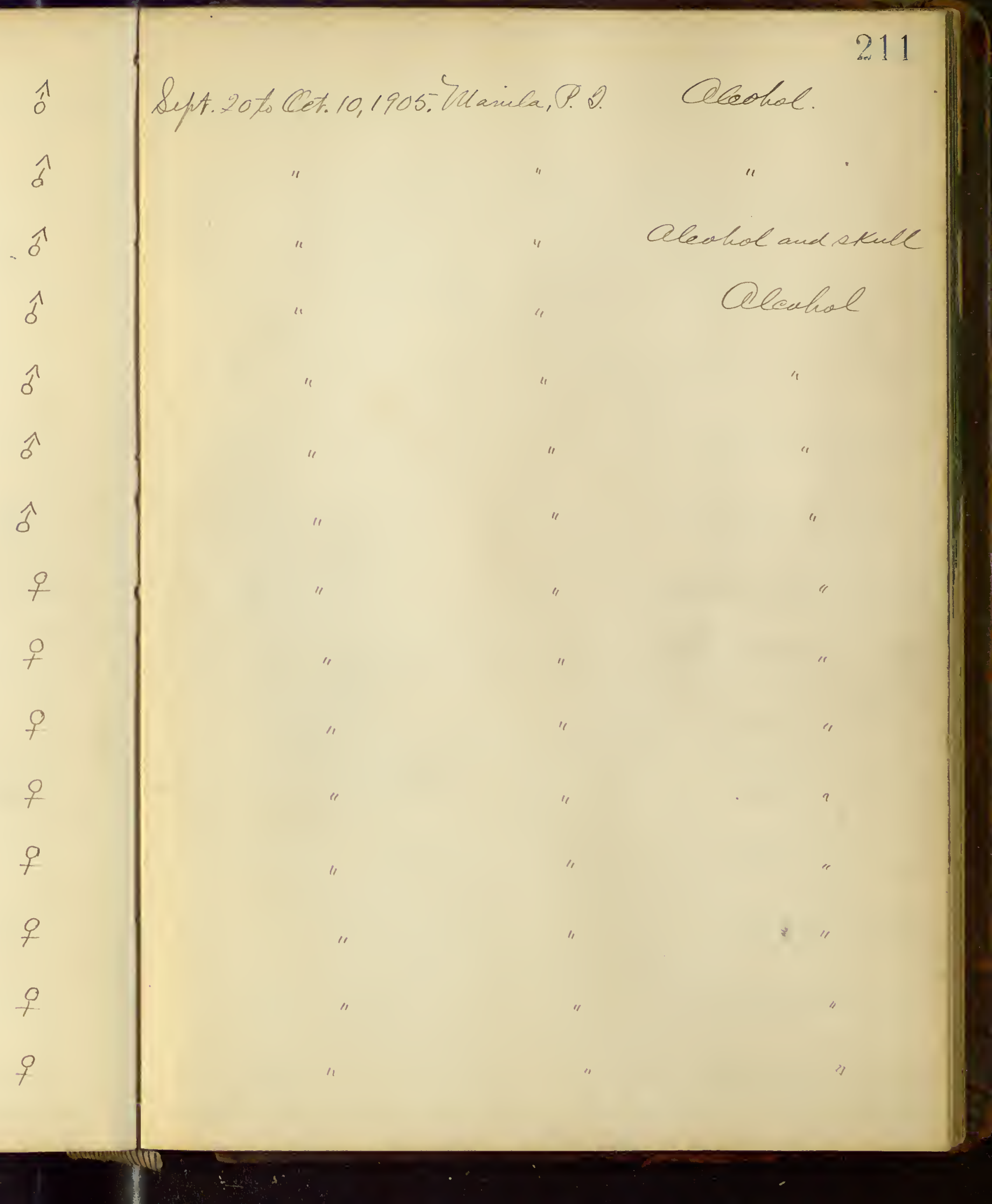




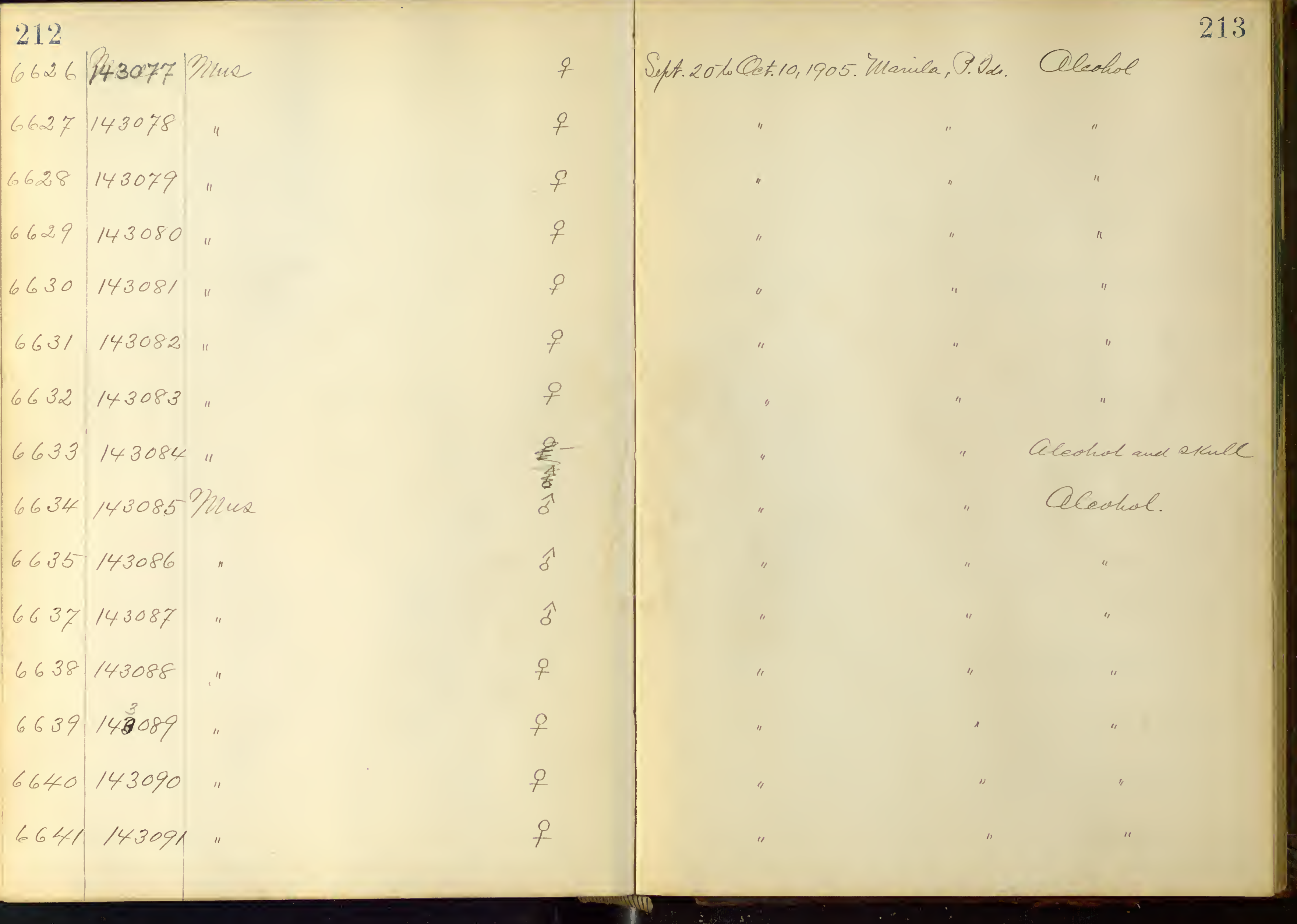




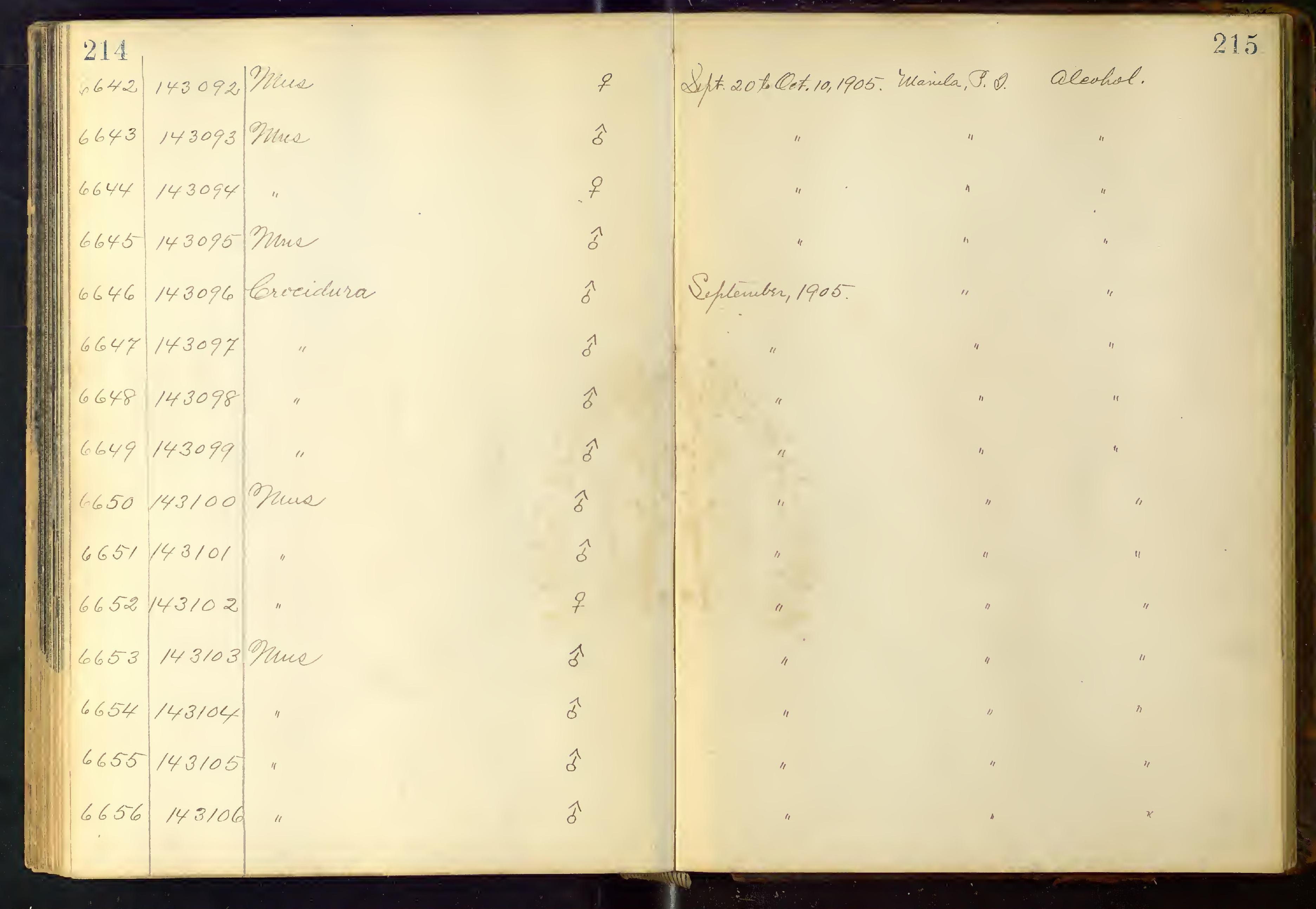









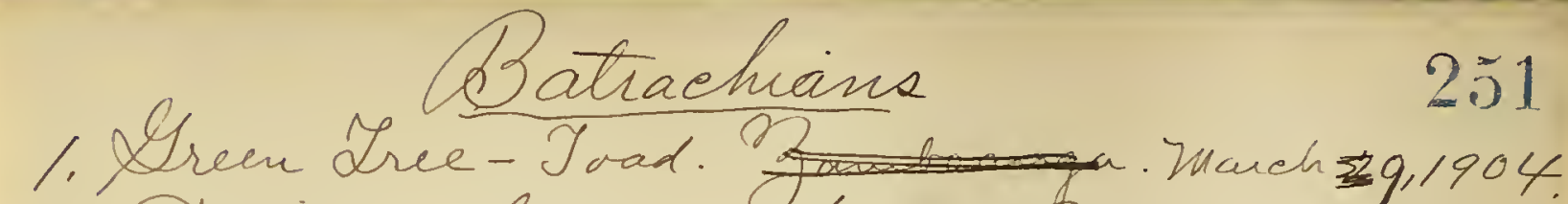
Dried in bstancal frees. Sint by mail. Barak, near sferenaja, lestabato Vallen, Min= =danno, P. d. Buareh 9,1904. 
264 Pants shiphed to U. s. nat. Unems

1. Friet frow Mnariveles, Kuzon, O. C. Ang, 1903.

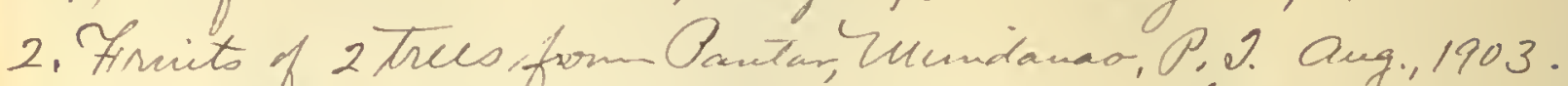

3. "Lart of fuessed sfecmien prewoish, sent. Sicam. Gulp21,1903. 4. Inv segmente of frintage of hesk Pineapple from

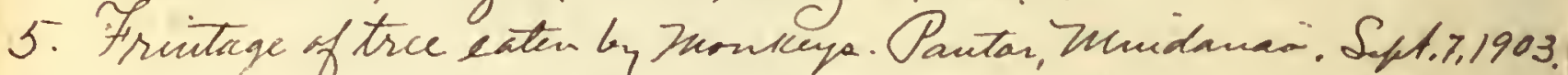
Common Mnidanao friets are: Mango, Banana,

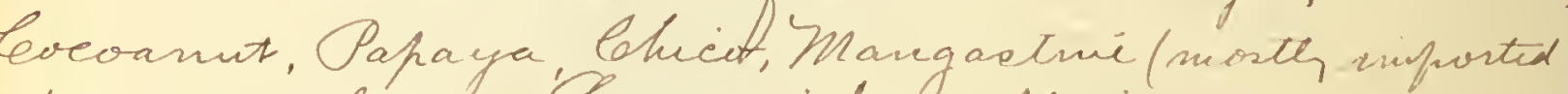
from Golo, Sulu 1. 'Sanzoni, Strape Frrit

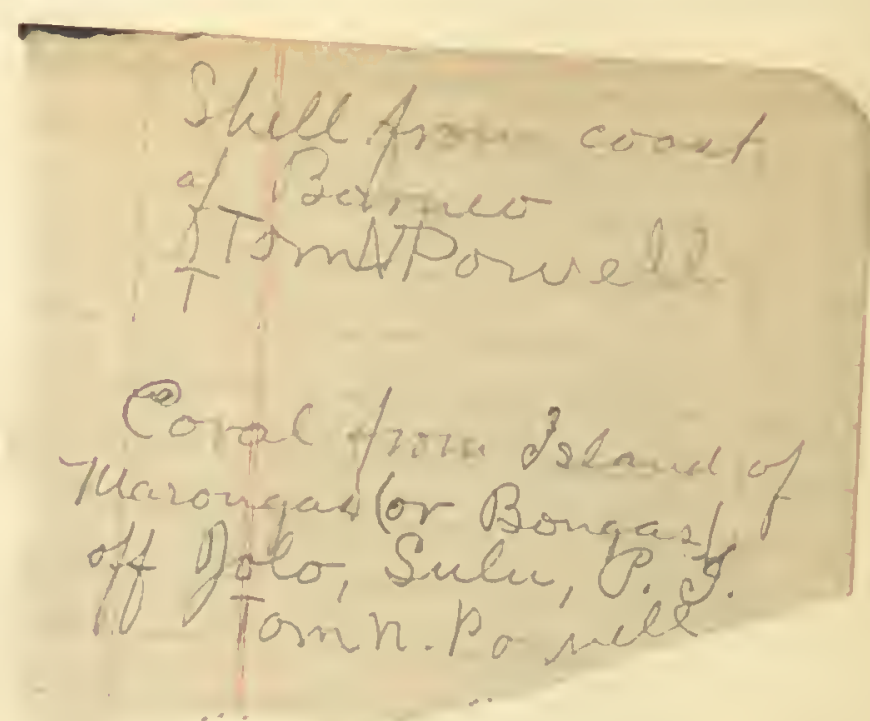

\section{Shelle shipfed to U. Q. Kat. Uner}

Large nantilas shell. Mariveles, Luzon, P. . . Ang. 4, 1903. 2 Cigar bor filled with land shells from Pantar, Mindanaot, P. 2. Ougust, 1903

3. Cigar bax contarimin a Sea Dollar from brach nerer Sliff Horse, Ran H rranaises, Calif. 'July 30,1903 Collectori of Finsa-water univalves Arom Lakci. Lanav, Thindanas. Oet. 200oft. Aug.30,1903. Package of large land suaits. Pantur. Quy.11903.

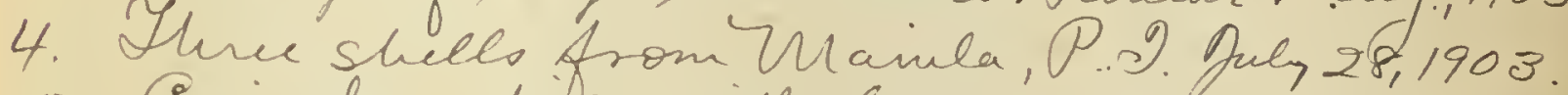
5. Cigar box filled with land shella and shelle gathered in the forast near Thariveles, Luzon, Ps?. Anguat 4, 1903 .

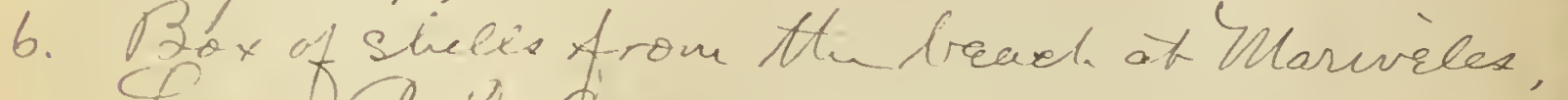
Lizon. O. Q. Ang. 4, 1903.

7. Dox of shelle? Ditto 8. Cigar box filled with shelle from Ganboanga,

9. Shells and Sea Dullur. from the leach at Dapitan, Mndarno, P. 9. Ceng. 9,1903 10. In Gox dilled with shells form the Diland 


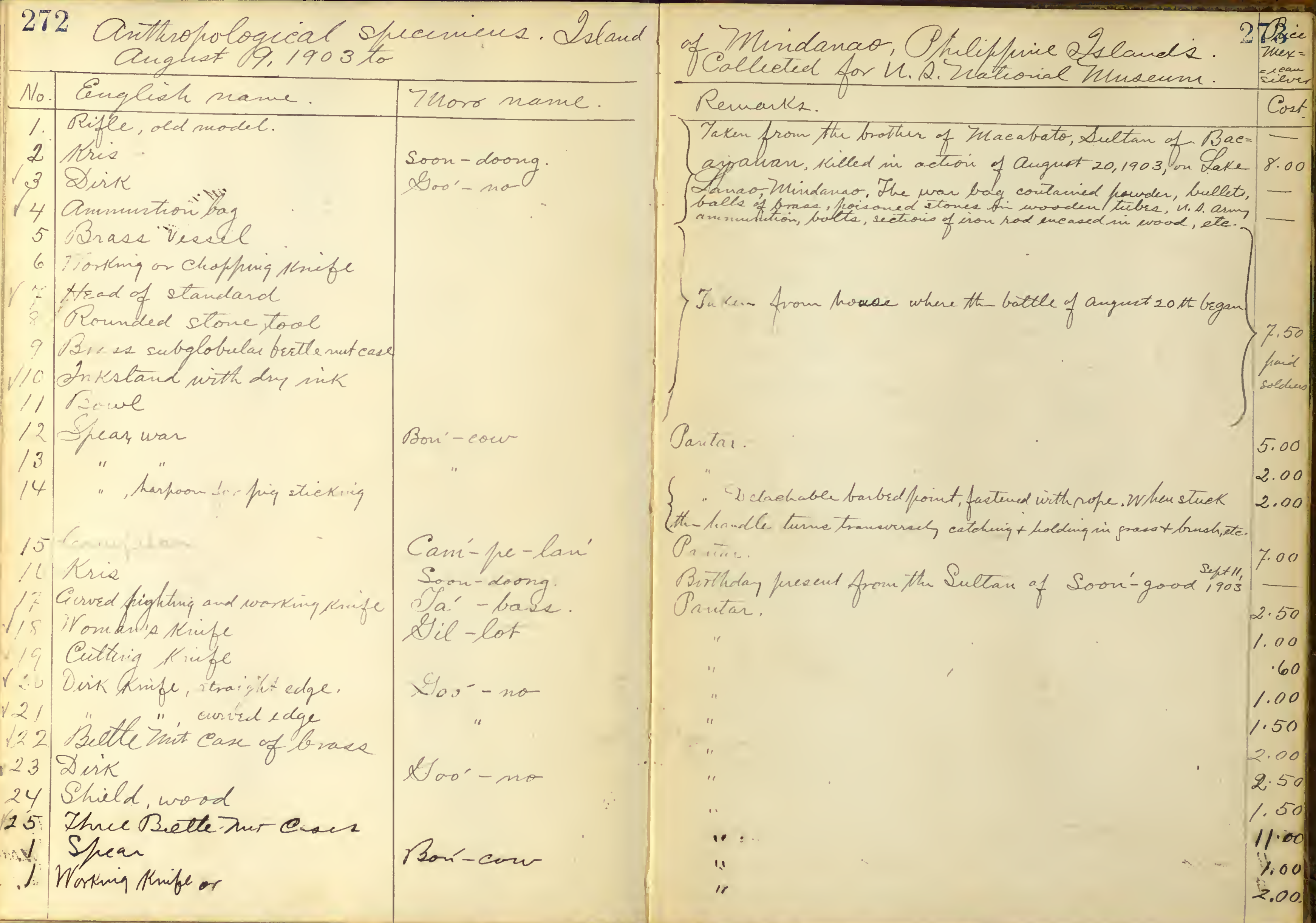




\section{1}

Durnig the Becond Bulu Expedition, on Nombre /7, 1903, thvo Moro ehildren, a girl maned of aratea and a boy named Y ahmat, whose father was killed, wre earried out of the swampinean Crater Sake, where heavy fithtinig wrre sent to Golo, Sulu, and freaced under the charge of toolonel Leott, military forrsuor \&'sulir. Thir father's name, Jarong; mothers, Pula. Athefeliedren told the intirfireter that their younger brotter was with his mother at Pajzlemà Arassan's place Odqar D. Mearus (Chif Surgeon of Second Sulu Expechition) 


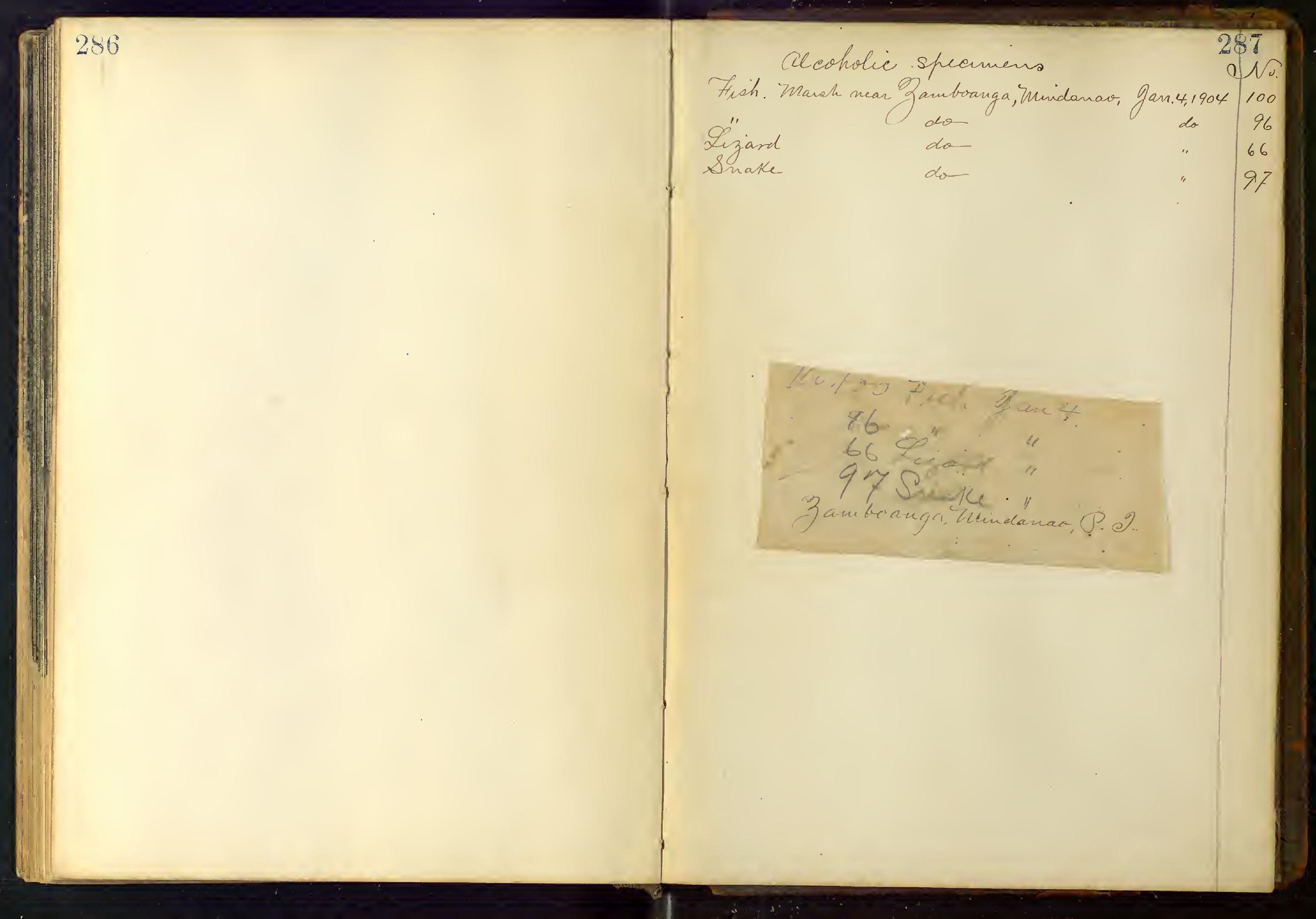




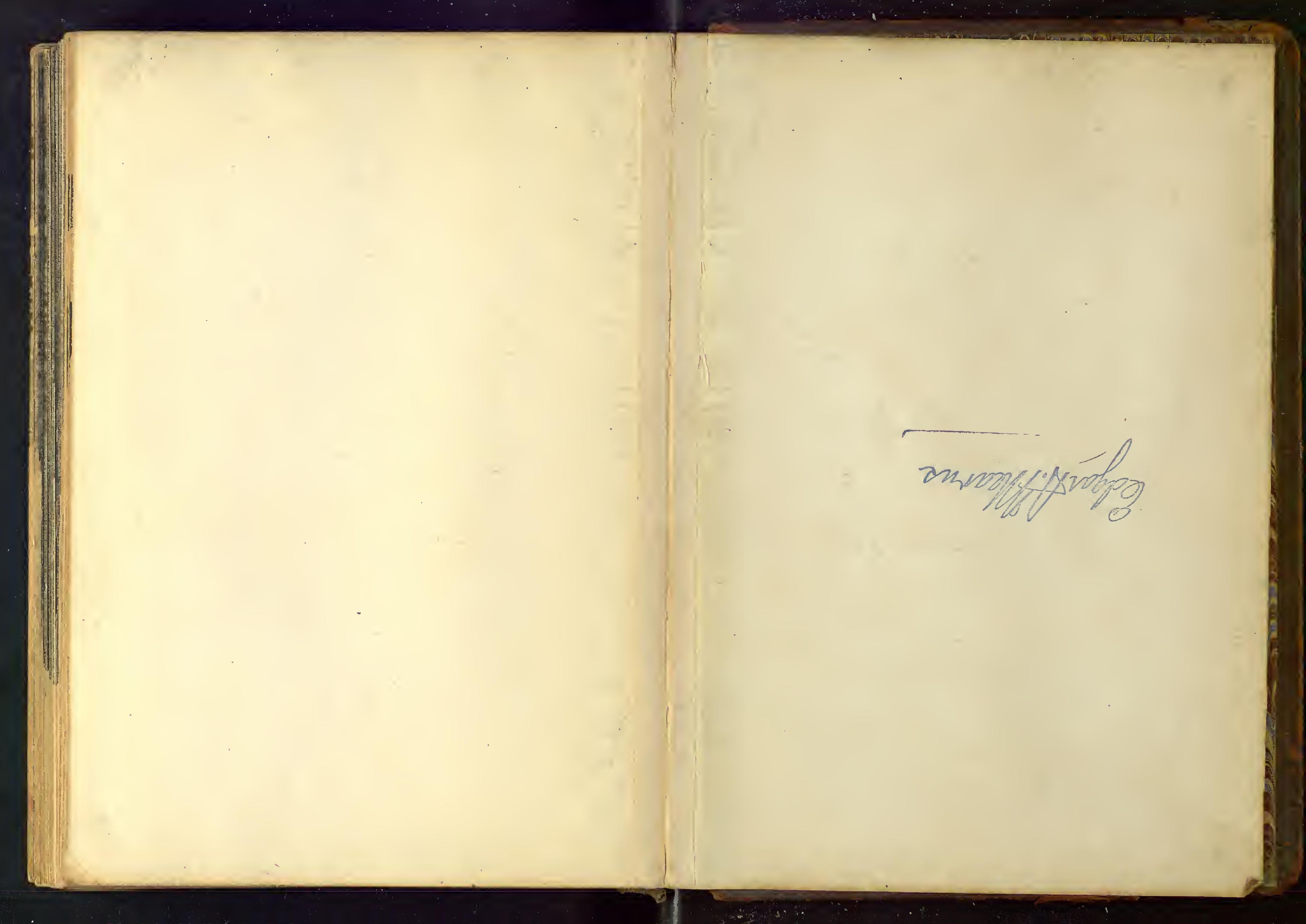


Mammal Data fordr. Dyou.

Qn the Cateel River, E. Unidanas, Oetober 5, 1906, I) noted: "Daw no nammale but Mhonkeyp. Surenal Maod shot a fine male. "Doublese the sull wax kept. (? hetal tag) no. 3719).

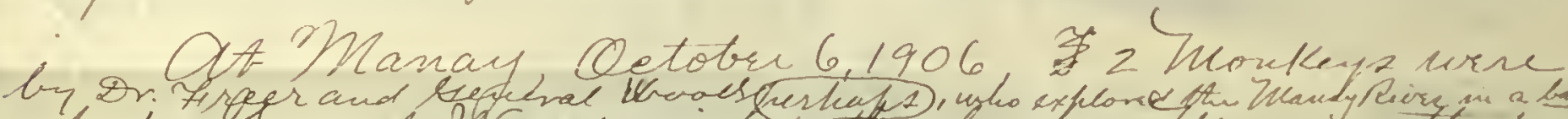

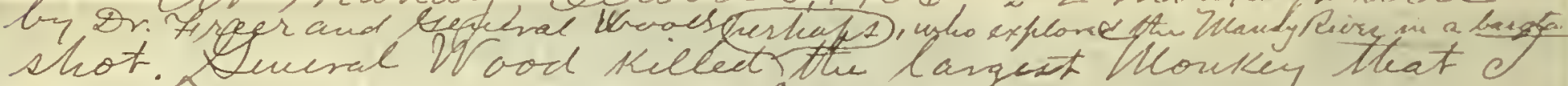
shot. Sueral have seen in the Philiffinic Oslands. I hewr sotid Dhat it wrighed exaethy 20 founde and that bott" s kulle

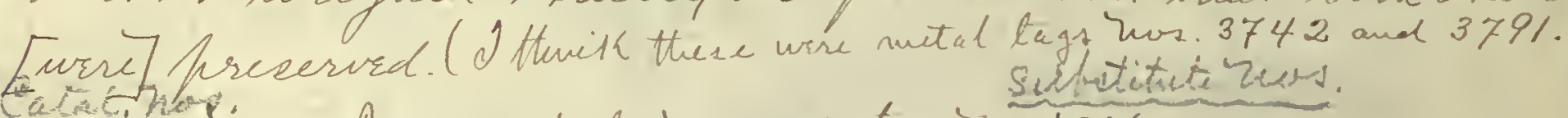

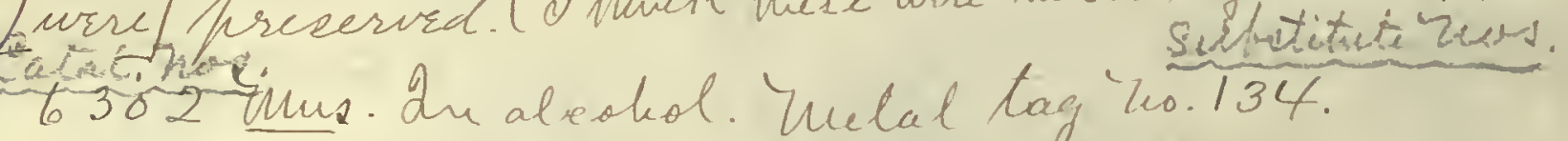

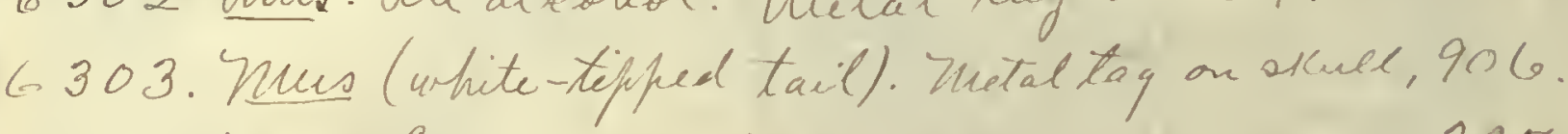
6304 . Mus Small. Sf jir.

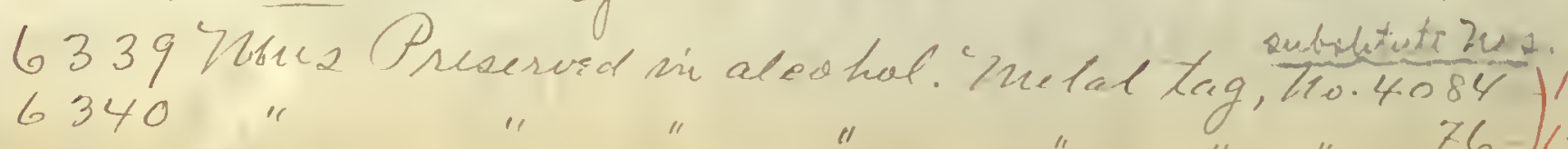

6341

6342

6343

6344

6345

$76-144926$
$74-144927$
$75-144928$
$73-144929$
$77-144930$
$78-144931$


Pejic paue 2

5

Note. The large for Dr. Syon.

Note. In large mumal sululls from Llocos horte. Provinee, Luzon 2or, P.? had paper tags. Dest they bs lost I attached eheek numbers on metal tage as foclows: -

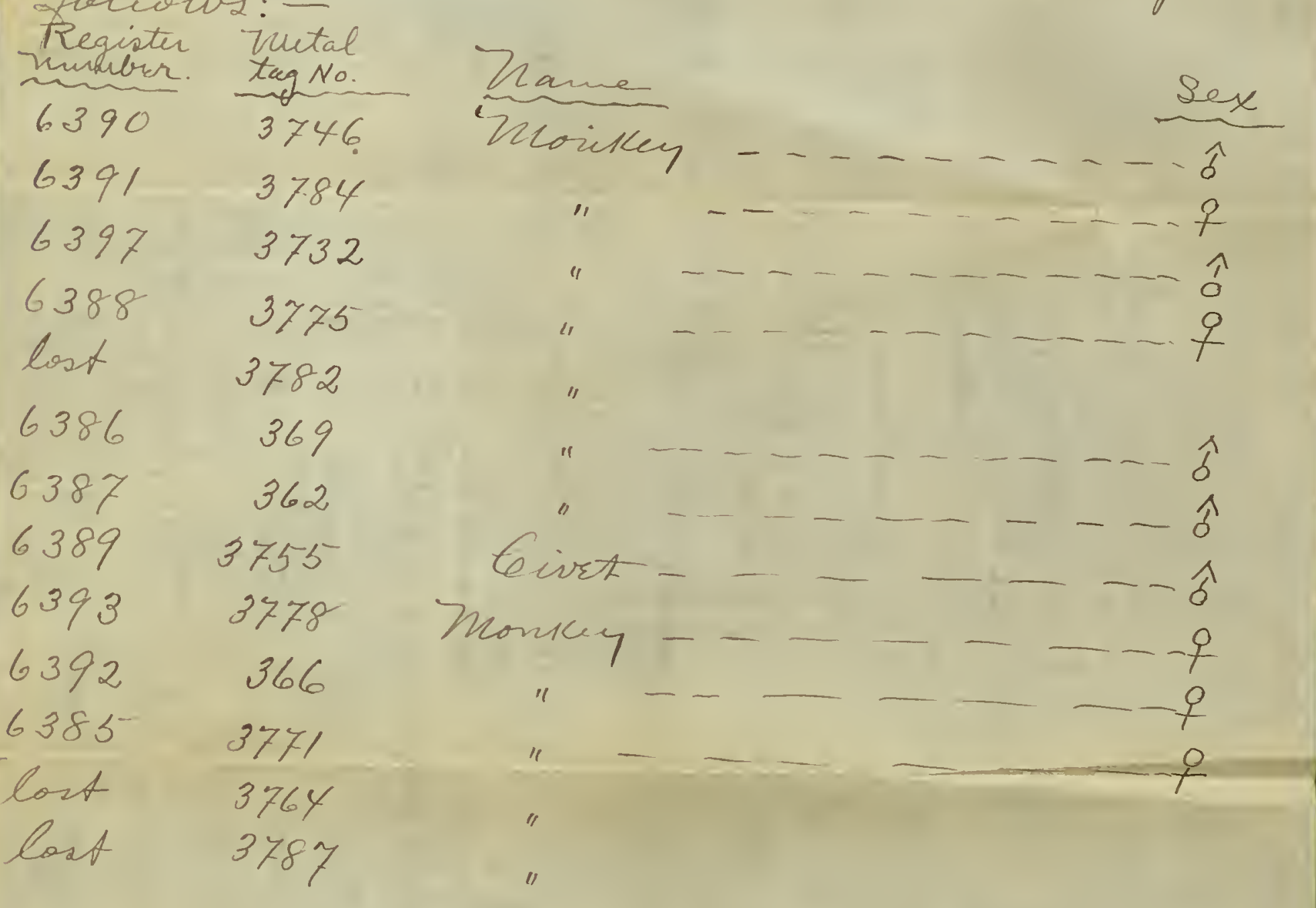




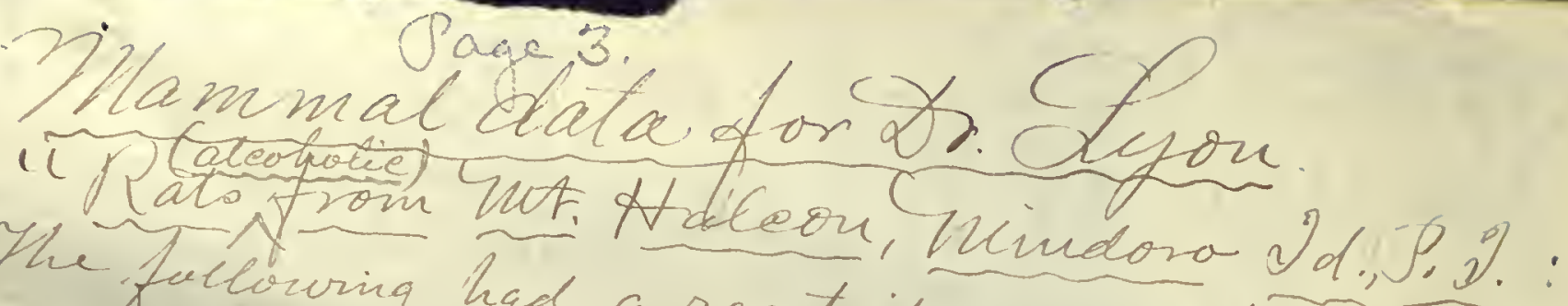

"The following had a rag tied around hind foot, and came from last camp, returning, at Banyan clearing on bill, 12 miles from Subadar, limed vo,

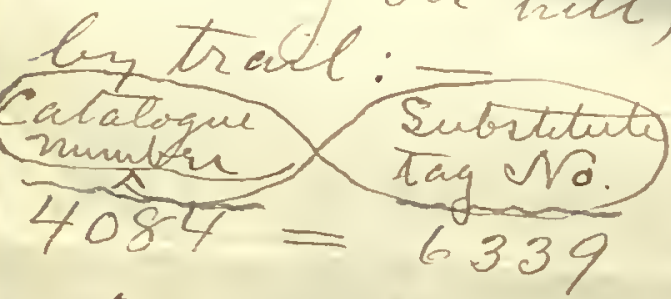

$$
76=6340
$$$$
74=6341
$$$$
75=6342
$$

$v 7=6343$

$7^{7}=6344$

$\sqrt{78}=6345$
Sextaqe

9 ad. $(144925)$
$q-(144926)$

$q-(144927)$

$q-(144928)$

$9-(114929)$

q $-(144930)$

it -(144931) its. "foot for a mark: -

$82=$ horace not registered. Gad (14492)

"The 3 following wire fut in ales bat by Colons, at

camp on 2 nd hill (altitide2250 fut):-

$72=6299$

$81=6300$

$$
\begin{aligned}
& \hat{d} \text { ad. }-(144932) \\
& \hat{\sigma} a d .-(144933) \\
& \text { fad.- }(144934)
\end{aligned}
$$

$134=6302$ g ing came from higher wt the mon vain:

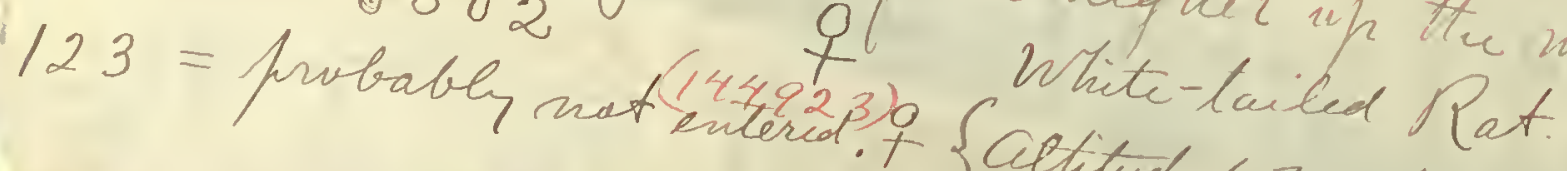

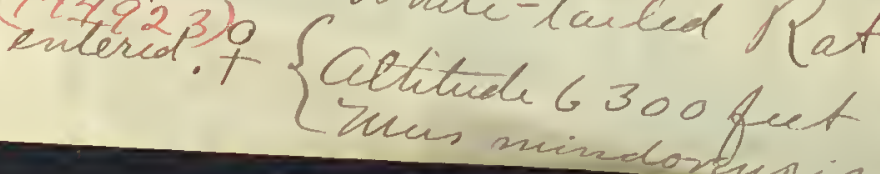


Hemal data 4 age 4

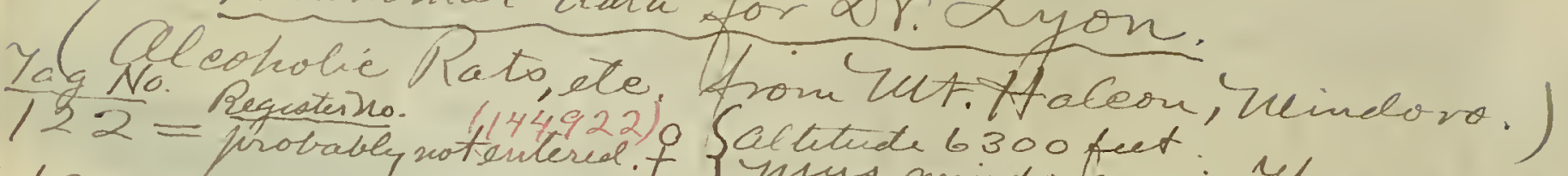

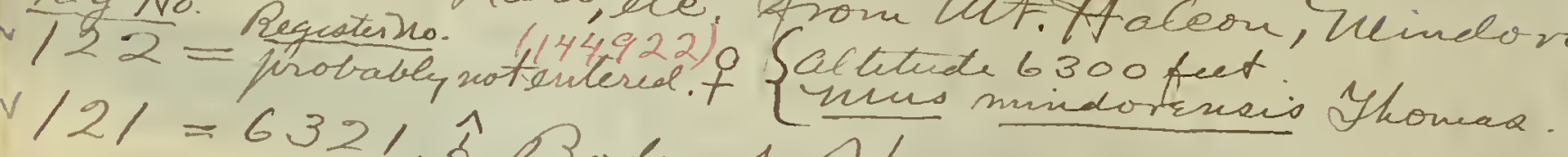

$v / 21=6321 . \hat{8}$ Body of Shrew.

$\checkmark 79=6313006323$. Shrew, entire

$120=6313$ or 6323 do 


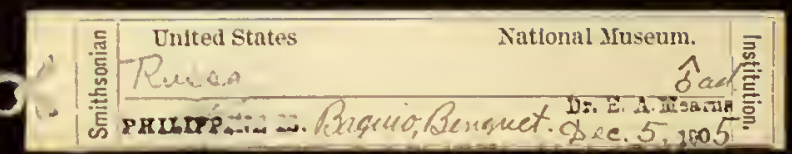




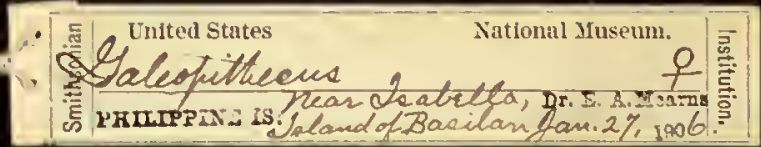


Harne ard stien of body. 
Tot frequant. Shot frome a hegl do swingming vire connecting thees isi

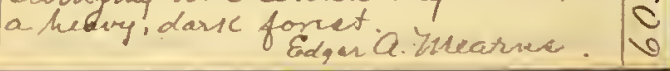


THE BRUGUIEPI;-BhBCOCK MINSTREL OCLPANYMLIIITED. Positively first and last performanco (By request) U.S.A.T."SHER:LAN", Friday, September I3, I9C7, at 9.15 P.M. (IIFE PRESIRVTRS FURIISHID WITF EVRRY SEAT).

\section{Part I.}

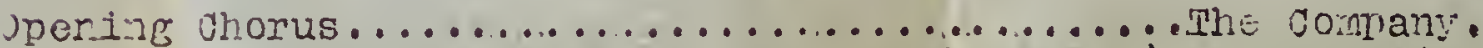

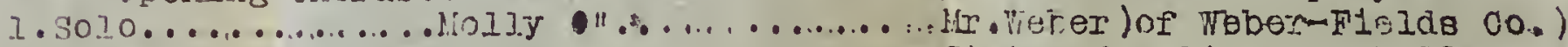

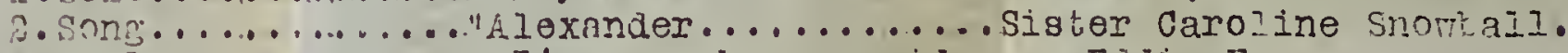

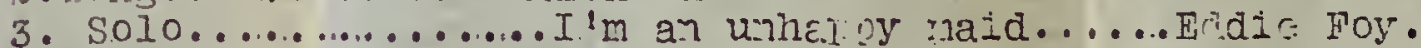

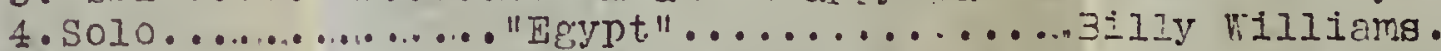

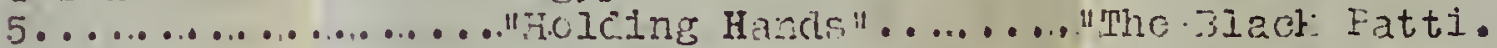

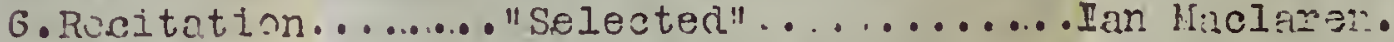

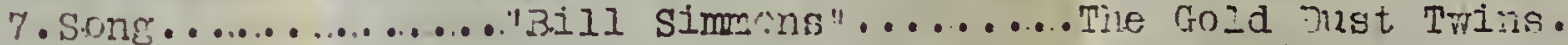

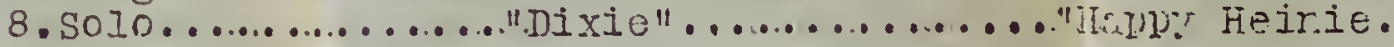

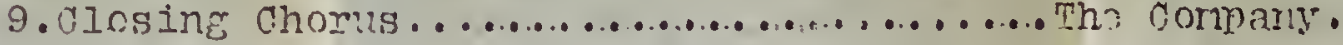

Pant, II.

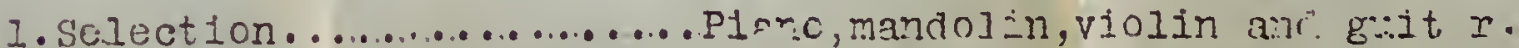

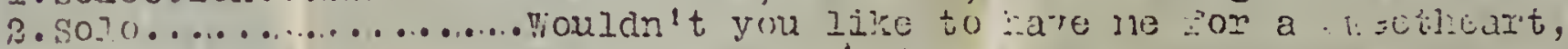
lia. icistus tringinn.

3......... İttile talk rn Transport sorvice..im. Jociloy.

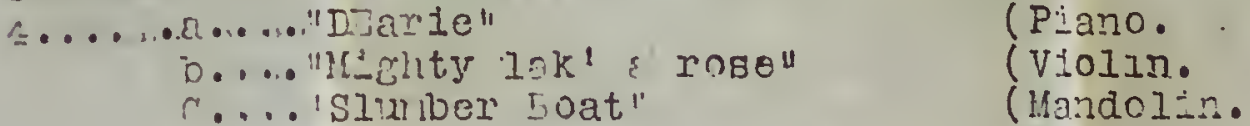

Pant III.

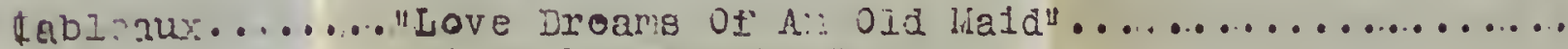

a. "First Love" The Fa"iler".

b. "Calf is ovel".

o. "Hen Ideal".

c. "The "Atinse Iclol".

e. "Iile Groom That Filied".

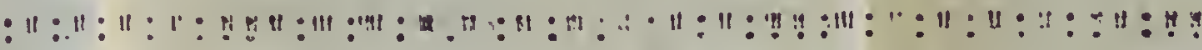

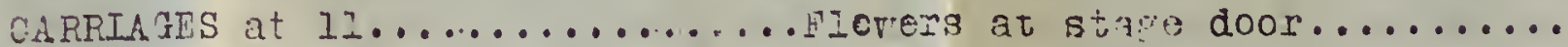

